Research Article

\title{
Two-Scale Network Dynamic Model for Energy Commodity Processes
}

\author{
Olusegun M. Otunuga $\mathbb{D}^{1}$ and Gangaram Ladde ${ }^{2}$ \\ ${ }^{1}$ Department of Mathematics, Marshall University, One John Marshall Dr, Huntington West Virginia 25755, USA \\ ${ }^{2}$ Department of Mathematics and Statistics, University of South Florida, 4202 E. Fowler Avenue, Tampa, Florida 33620, USA \\ Correspondence should be addressed to Olusegun M. Otunuga; otunuga@marshall.edu
}

Received 23 August 2019; Revised 9 January 2020; Accepted 3 February 2020; Published 20 April 2020

Academic Editor: Ciro Aprea

Copyright (c) 2020 Olusegun M. Otunuga and Gangaram Ladde. This is an open access article distributed under the Creative Commons Attribution License, which permits unrestricted use, distribution, and reproduction in any medium, provided the original work is properly cited.

\begin{abstract}
In this work, we examine the relationship between different energy commodity spot prices. To do this, multivariate stochastic models with and without external random interventions describing the price of energy commodities are developed. Random intervention process is described by a continuous jump process. The developed mathematical model is utilized to examine the relationship between energy commodity prices. The time-varying parameters in the stochastic model are estimated using the recently developed parameter identification technique called local lagged adapted generalized method of moment (LLGMM). The LLGMM method provides an iterative scheme for updating statistic coefficients in a system of generalized method of moment/observation equations. The usefulness of the LLGMM approach is illustrated by applying to energy commodity data sets for state and parameter estimation problems. Moreover, the forecasting and confidence interval problems are also investigated (U.S. Patent Pending for the LLGMM method described in this manuscript).
\end{abstract}

\section{Introduction}

Understanding the economy evolution in response to structural changes in the energy commodity network system is important to professional economists. The relationship between the different energy sources and their uses provide insights into many important energy issues. The quantitative behavior of energy commodities in which the trend in price of one commodity coincides with the trend in prices of other commodities has always raised the question of whether there is any relationship between prices of energy commodities. If there is any relationship, then what comes to mind is the extent to which one commodity influences the other. Petroleum, natural gas, coal, nuclear fuel, and renewable energy are termed as primary energy components of the energy goods network system because other sources of energy depend on them. Natural gas is usually found near petroleum. Hence, natural gas and crude oil are rivals in production and substitutes in consumption, and energy theory suggests that the two prices should be related. The electric power sector uses primary energy such as coal to generate electricity, which makes electricity a secondary rather than a primary energy source. According to the US Energy Information Administration (EIA), the major energy goods consumed in the United States are petroleum (oil), natural gas, coal, nuclear, and renewable energy. The majority of users are residential and commercial buildings, industry, transportation, and electric power generators. The pattern of fuel usage varies widely by sector [1]. For example, $71 \%$ of total petroleum oil provides $93 \%$ of the energy used for transportation; $23 \%$ of total petroleum oil provides $17 \%$ of energy used for residential and commercial use; $5 \%$ of total petroleum oil provides $40 \%$ of energy used for industrial use; but only $1 \%$ of total petroleum oil provides about $1 \%$ of the energy used to generate electric power, whereas coal provides $46 \%$ of the energy used to generate electric power and natural gas provides $20 \%$ of the energy used to generate electric power. This analysis suggests that the strength of interactions between coal and electricity will be stronger than that of crude oil and electricity, or natural gas and electricity.

Energy price forecasts are highly uncertain. We might expect the price of the natural gas and crude oil to follow 


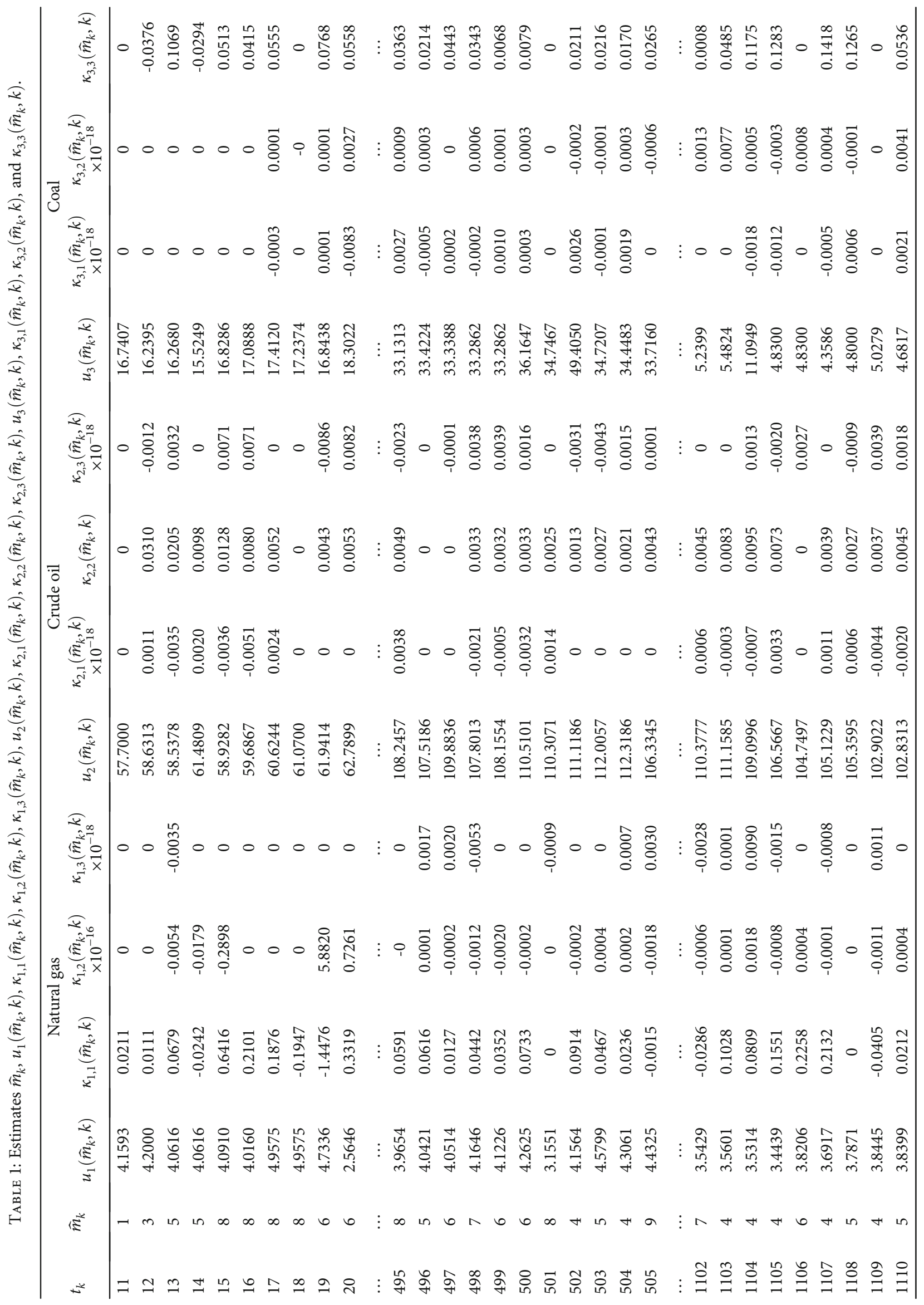




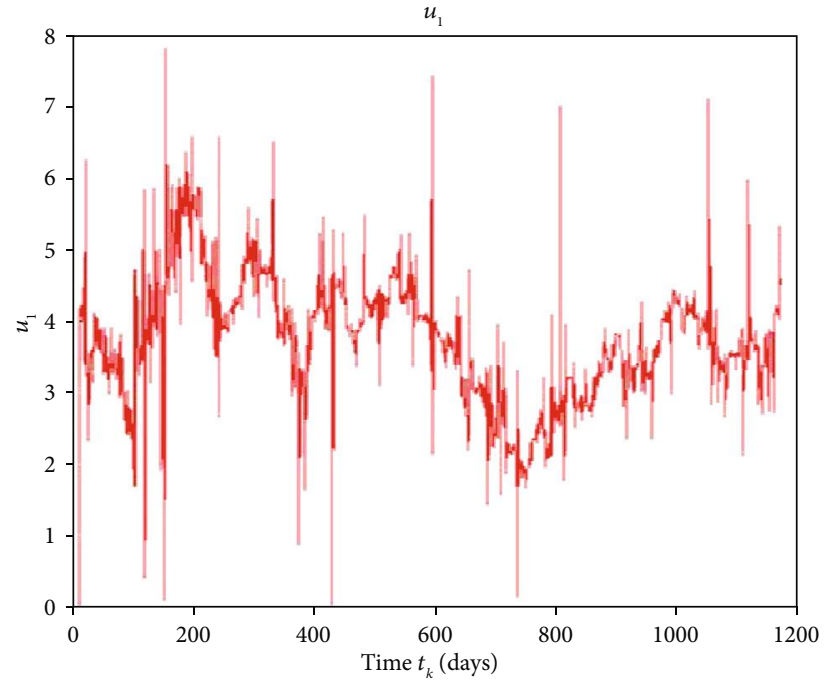

(a)

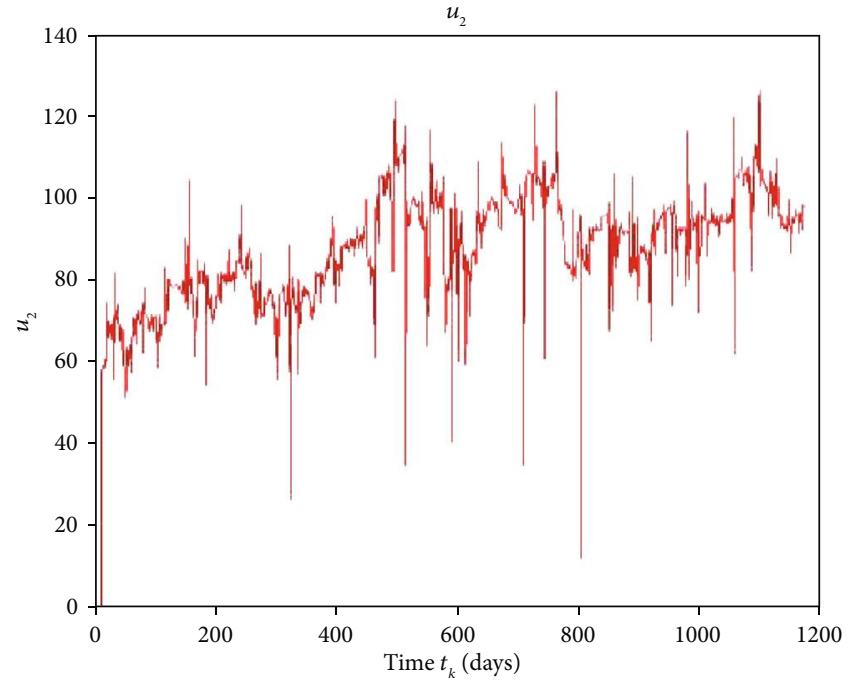

(b)

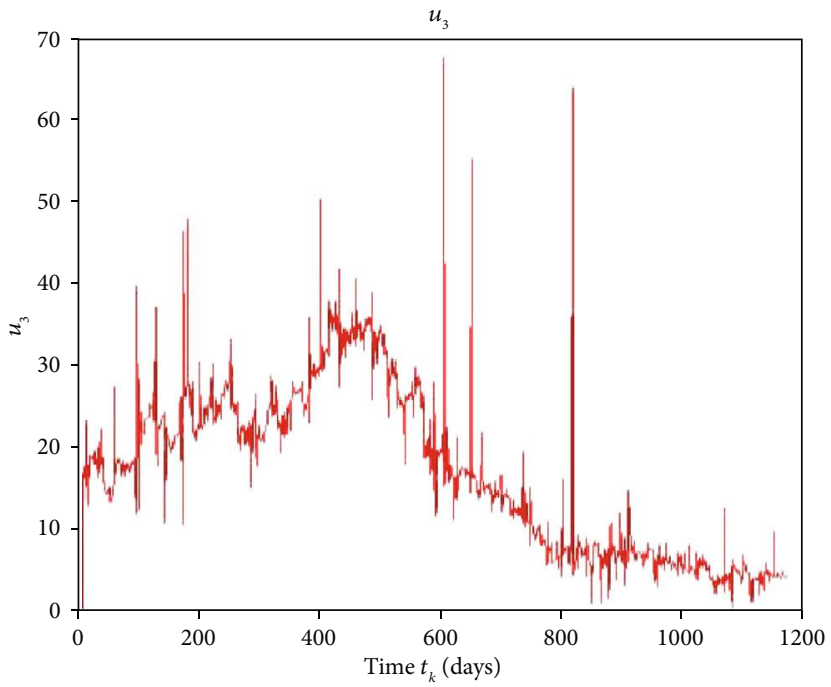

(c)

Figure 1: The graph of mean level $u_{1}\left(\widehat{m}_{k}, k\right), u_{2}\left(\widehat{m}_{k}, k\right)$, and $u_{3}\left(\widehat{m}_{k}, k\right)$ for natural gas, crude oil, and coal, respectively.

the same trend because they are often found mixed with oil in oil wells and also of the fact that natural gas is often used in petroleum refining and exploration. Recently, Ramberg and Parsons [2] showed that the cointegration relationship between natural gas and crude oil does not appear to be stable through time. They claimed that though there is cointegration between the two energy prices, there are also recurrent exogeneous factors such as seasonality, episodic heat waves, cold waves, and supply interruption from the hurricane affecting the trends in the prices. Brown and Yücel [3] also observed that the price of natural gas pulled away from oil prices in 2000, 2002, 2003, and late 2005. Oil prices are influenced by several factors, including some that have mainly short-term impacts and other factors, such as expectations about the future world demand for petroleum, other liquids, and production decisions of the Organization of the Petroleum Exporting Countries (OPEC) [1]. Supply and demand in the world oil market are balanced through responses to price movement with considerable complexity in the evolution of underlying supply and demand expectation process. For petroleum and other liquids, the key determinants of long-term supply and prices can be summarized in four broad categories [1]: the economics of non-OPEC supply, OPEC investment and production decisions, the economics of other liquids supply, and world demand for petroleum and other liquids.

An understanding of how changes in the price of one energy commodity are expressed in terms of other energy commodity is needed. This would prove to be useful in predicting price behavior over the long run and further facilitates profit-maximizing processes. To check if there is really indeed a relationship between energy commodities, the need to be able to create a model which explains such commodity price relationship over short- and long-time intervals arises. The relationships between energy commodities have been addressed in [2-15]. The error correction model $[2-5,7,8]$ 


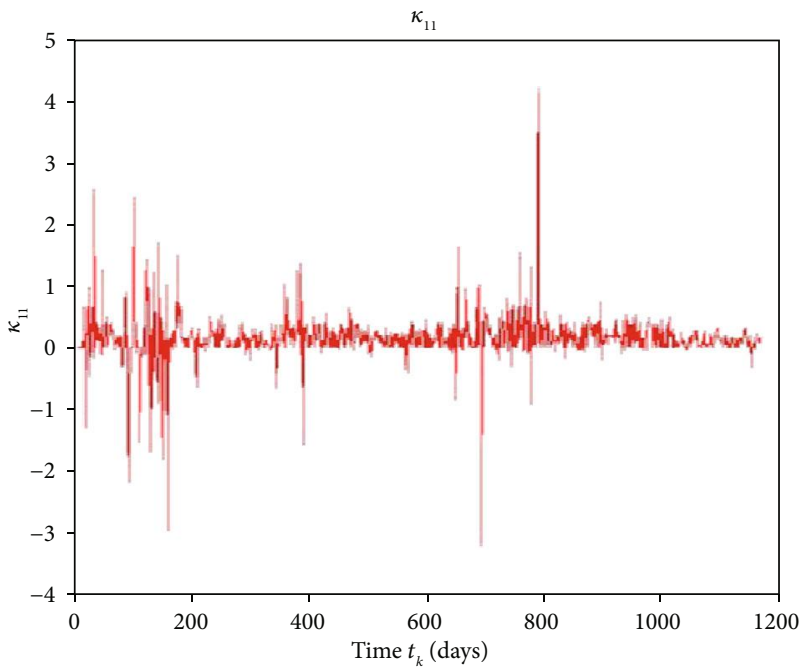

(a)

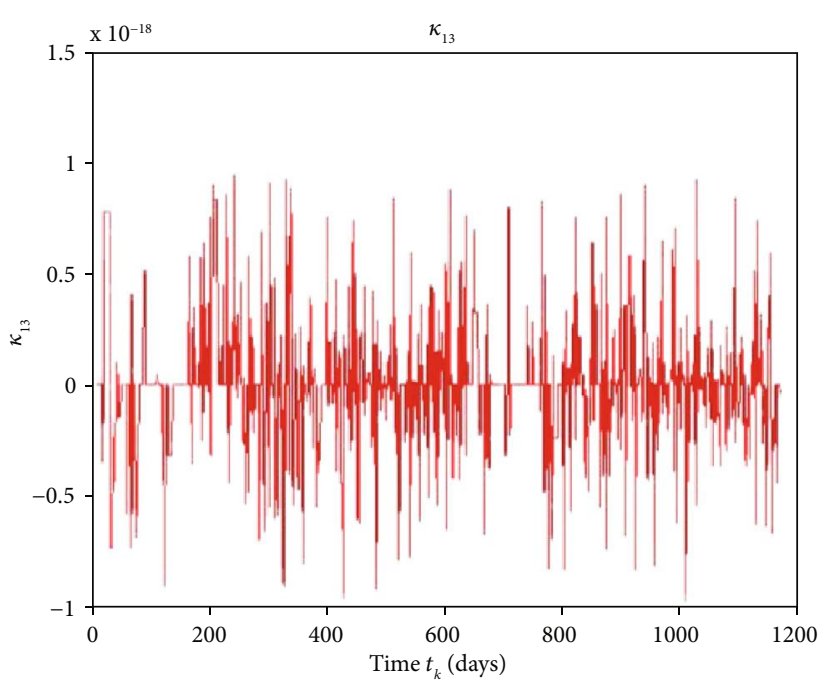

(c)

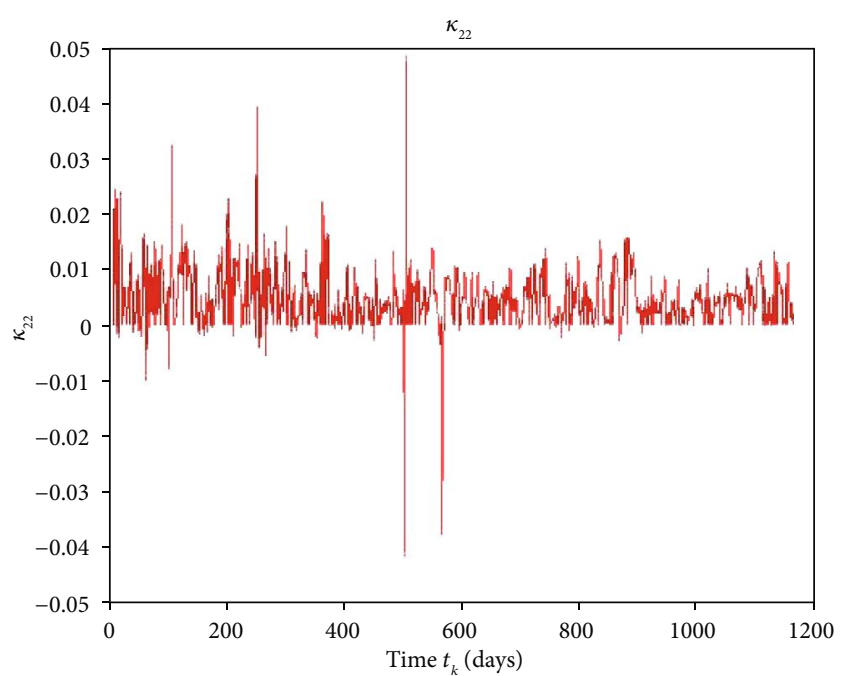

(e)

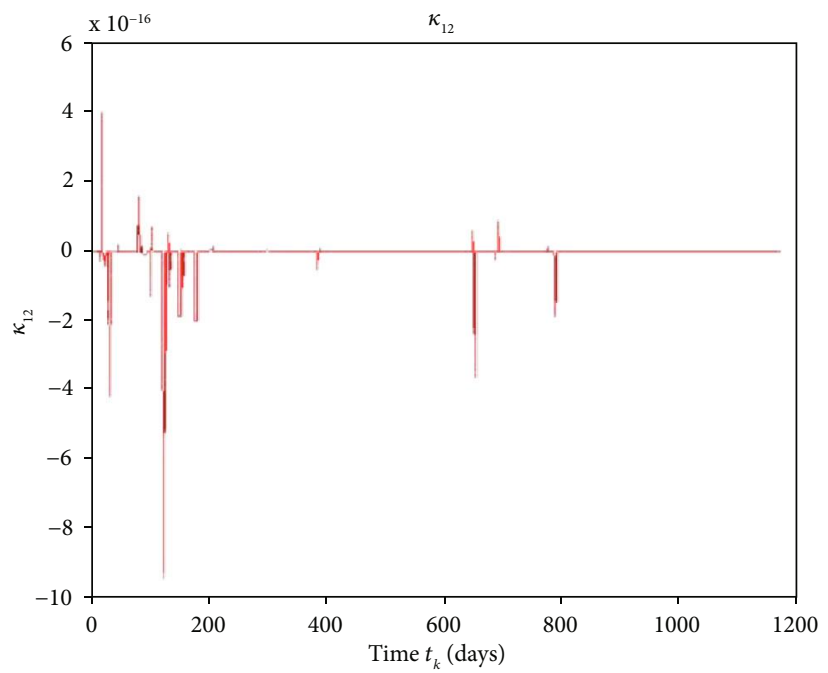

(b)

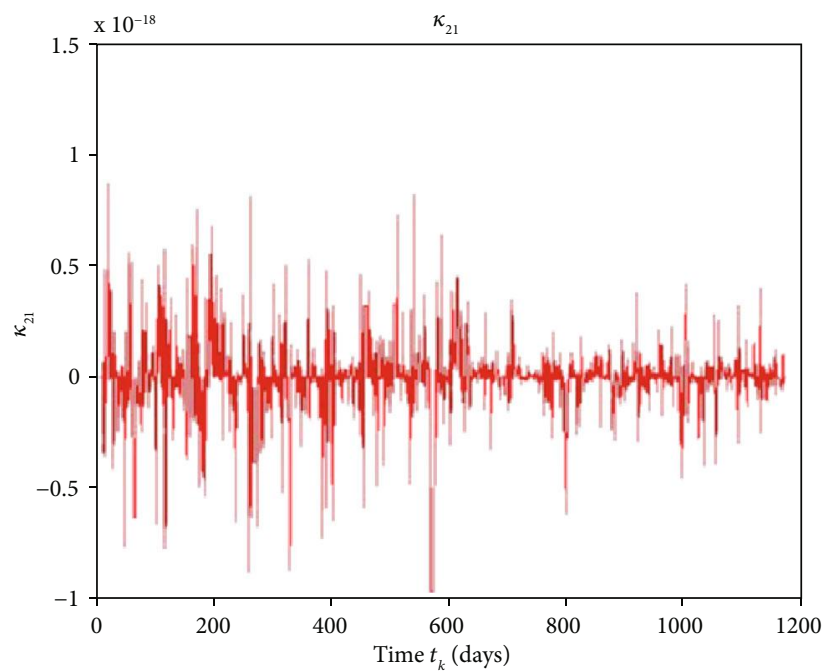

(d)

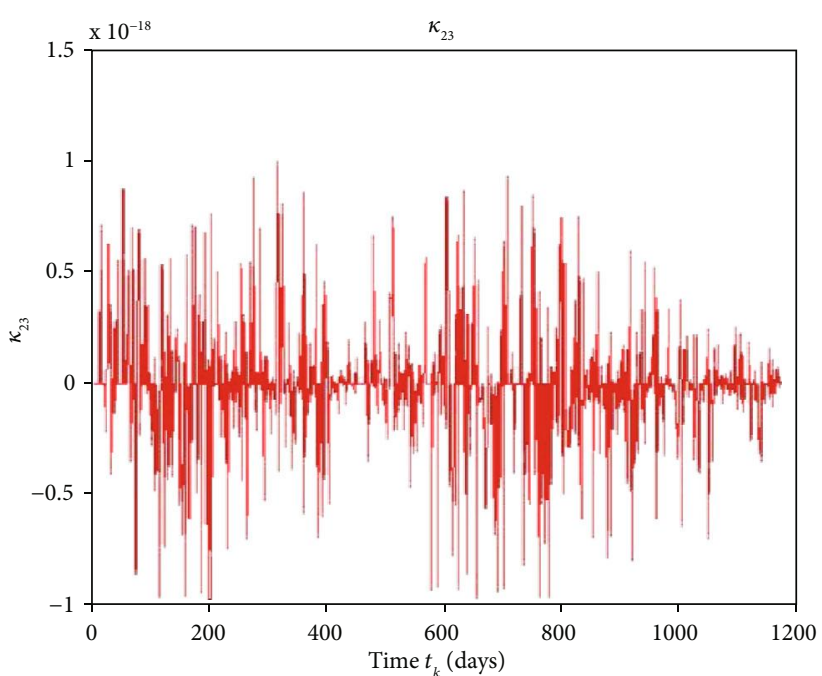

(f)

FIgUre 2: Continued. 


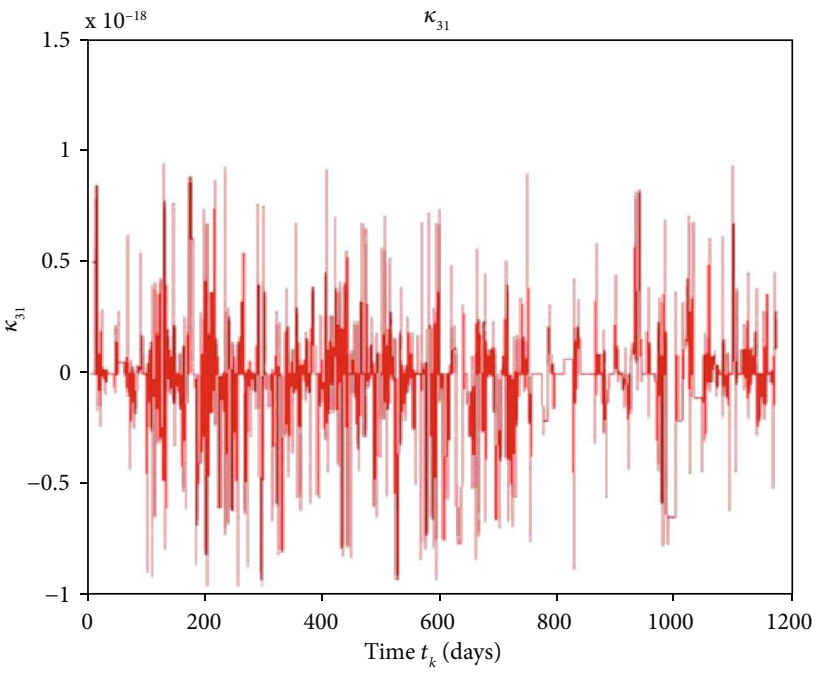

(g)

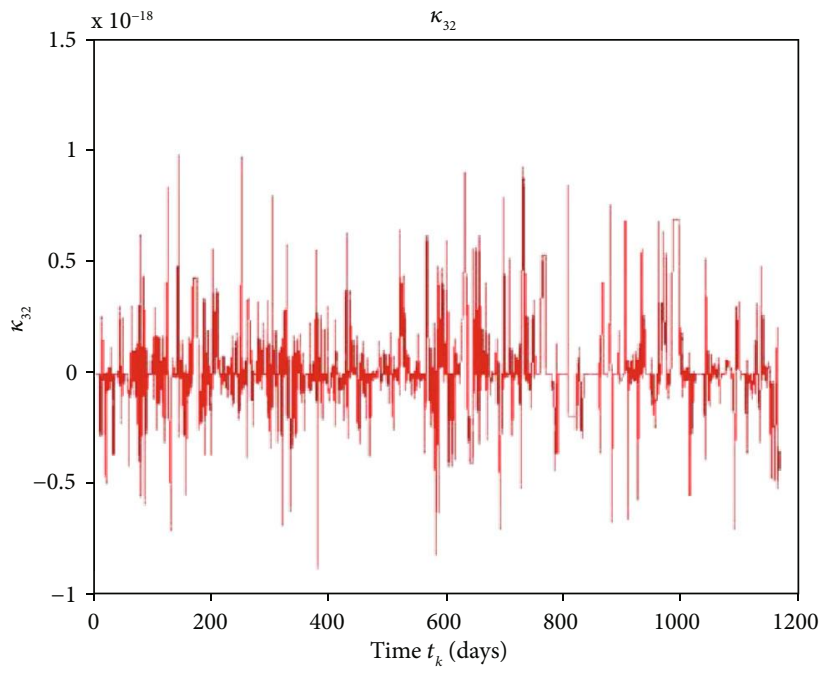

(h)

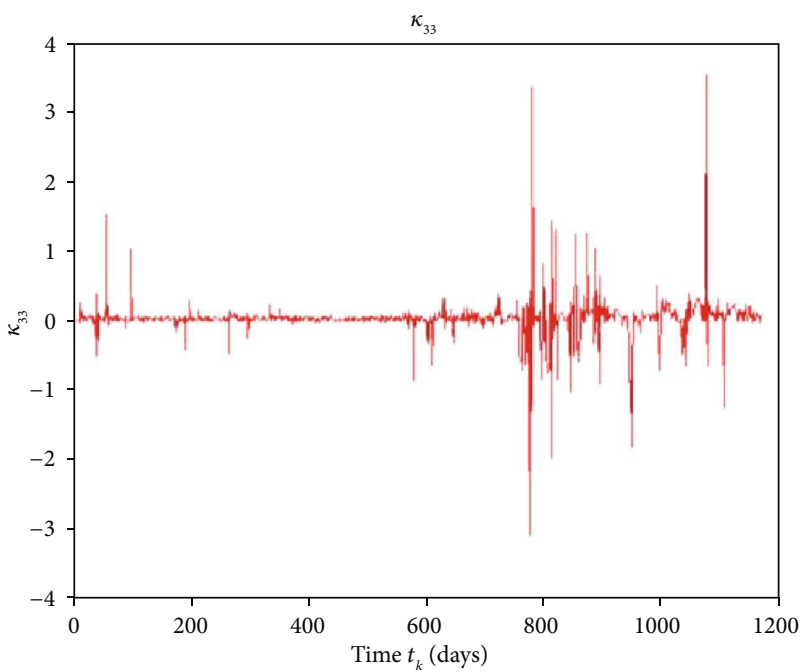

(i)

FIGURE 2: The graph of interaction coefficients $\kappa_{1,1}\left(\widehat{m}_{k}, k\right), \kappa_{1,2}\left(\widehat{m}_{k}, k\right), \kappa_{1,3}\left(\widehat{m}_{k}, k\right), \kappa_{2,1}\left(\widehat{m}_{k}, k\right), \kappa_{2,2}\left(\widehat{m}_{k}, k\right), \kappa_{2,3}\left(\widehat{m}_{k}, k\right), \kappa_{3,1}\left(\widehat{m}_{k}, k\right), \kappa_{3,2}\left(\widehat{m}_{k}, k\right)$, and $\kappa_{3,3}\left(\widehat{m}_{k}, k\right)$.

is the most commonly used model by authors to describe the relationship between energy commodities. Moreover, Hartley et al. [7] have concluded that the U. S. natural gas and crude oil remain linked in their long-term movements. In addition, it is exhibited that there is strong evidence of a stable relationship between these two energy commodities which are affected by short-run seasonal fluctuations and other factors. The rule of thumb [7] has long been used in the energy industry to relate the natural gas prices to crude oil prices. The rule denoted by the 10 -to- 1 rule states that the price of natural gas is one-tenth of the price of crude oil prices. Similarly, the 6-to- 1 rule states that the price of natural gas is one-sixth of the price of crude oil. It has been examined by Brown and Yücel [3] that these two rules do not perform well when used to assess the relationship between US natural gas price and West Texas Intermediate (WTI) crude oil price for the past $20^{+}$years. Moreover, their errorcorrecting model specifies the relationship between the two commodities. Using this model, they show that when certain factors are taken into account, movements in crude oil prices can shape the price of natural gas. Vezzoli [9] in his work applies an ordinary least squares (OLS) regression on the log of natural gas and crude oil prices. Using this model, he was able to conclude that there is a relationship between natural gas and crude oil prices. Bachmeir and Griffin [4] showed that crude oil, coal, and natural gas in the United States have weak cross-cointegration using the error correction model. Ramberg and Parsons [2] show that any simple formula between natural gas and crude oil prices will leave a portion of the natural gas price unexplained. Furthermore, the relationship between natural gas and crude oil using a vector error correction model [2, 3] under the 
TABLE 2: Estimates $\delta_{1,1}\left(\widehat{m}_{k}, k\right), \delta_{1,2}\left(\widehat{m}_{k}, k\right), \delta_{1,3}\left(\widehat{m}_{k}, k\right), \delta_{2,1}\left(\widehat{m}_{k}, k\right), \delta_{2,2}\left(\widehat{m}_{k}, k\right), \delta_{2,3}\left(\widehat{m}_{k}, k\right), \delta_{3,1}\left(\widehat{m}_{k}, k\right), \delta_{3,2}\left(\widehat{m}_{k}, k\right)$, and $\delta_{3,3}\left(\widehat{m}_{k}, k\right)$.

\begin{tabular}{|c|c|c|c|c|c|c|c|c|c|}
\hline$t_{k}$ & $\delta_{1,1}\left(\widehat{m}_{k}, k\right)$ & $\begin{array}{c}\text { Natural gas } \\
\delta_{1,2}\left(\widehat{m}_{k}, k\right)\end{array}$ & $\delta_{1,3}\left(\widehat{m}_{k}, k\right)$ & $\delta_{2,1}\left(\widehat{m}_{k}, k\right)$ & $\begin{array}{r}\text { Crude oil } \\
\delta_{2,2}\left(\widehat{m}_{k}, k\right) \\
\end{array}$ & $\delta_{2,3}\left(\widehat{m}_{k}, k\right)$ & $\delta_{3,1}\left(\widehat{m}_{k}, k\right)$ & $\begin{array}{c}\text { Coal } \\
\delta_{3,2}\left(\widehat{m}_{k}, k\right) \\
\end{array}$ & $\delta_{3,3}\left(\widehat{m}_{k}, k\right)$ \\
\hline 11 & 0.0123 & 0.0012 & 0.0001 & 0 & 0.0223 & 0 & 0.0412 & 0 & 0.0022 \\
\hline 12 & 0.0024 & 0.0011 & 0.0121 & 0.0234 & 0.0245 & 0 & 0 & 0 & 0.0112 \\
\hline 13 & 0.0001 & 1.3425 & 1.7280 & 1.9811 & 0.9899 & 0.9731 & 0.6374 & 0.6374 & 0.0123 \\
\hline 14 & 0 & 1.1267 & 0.6027 & 2.3258 & 0.1213 & 3.9128 & 1.6564 & 1.6564 & 0.0004 \\
\hline 15 & 1.15260 & 0.4287 & 0.6210 & 2.3252 & 0.0006 & 0.5083 & 1.6650 & 1.6650 & 0.4565 \\
\hline 16 & 4.9354 & 0 & 0 & 2.3217 & 0.0120 & 1.1124 & 1.6724 & 1.6724 & 0.8762 \\
\hline 17 & 4.1360 & 0.0989 & 3.6877 & 1.6425 & 0 & 0 & 1.7719 & 1.7719 & 0 \\
\hline 18 & 3.0410 & 0.1527 & 0 & 1.3105 & 0.9167 & 1.3451 & 1.7630 & 1.7630 & 0 \\
\hline 19 & 2.7713 & 0 & 0 & 1.1052 & 0 & 3.3241 & 1.7400 & 1.7400 & 0 \\
\hline 20 & 2.8461 & 0.0012 & 0.2221 & 0.1196 & 5.1929 & 0 & 0.6532 & 0.9876 & 0.0082 \\
\hline$\cdots$ & $\cdots$ & $\ldots$ & $\ldots$ & $\ldots$ & $\ldots$ & $\cdots$ & $\ldots$ & $\ldots$ & $\ldots$ \\
\hline$\cdots$ & $\ldots$ & $\ldots$ & $\cdots$ & $\ldots$ & $\cdots$ & $\ldots$ & $\cdots$ & $\cdots$ & $\ldots$ \\
\hline 494 & 2.9961 & 0.0586 & 0 & 0.5529 & 0 & 0.42339 & 0 & 0 & 0.5187 \\
\hline 495 & 5.9059 & 0 & 0.0584 & 0.5488 & 0.8947 & 0 & 0.0017 & 0.0021 & 0.0001 \\
\hline 496 & 0.1121 & 0 & 0.6613 & 0.5767 & 0.9899 & 0 & 0.8763 & 0 & 0.9827 \\
\hline 497 & 1.1229 & 0.0095 & 0.0988 & 0.6499 & 5.8547 & 0 & 1.1317 & 1.1317 & 0.0012 \\
\hline 498 & 0.6946 & 0.0101 & 0 & 0 & 5.8298 & 0.0320 & 1.0294 & 1.0294 & 0.0321 \\
\hline 499 & 0.7353 & 0.0066 & 0.0384 & 0 & 5.7180 & 0.0330 & 0.7317 & 0.7317 & 0.0431 \\
\hline 500 & 1.7509 & 0.0069 & 0.0283 & 0.4307 & 5.6133 & 0.0413 & 0.4826 & 0.4826 & 0.0783 \\
\hline 501 & 2.1299 & 0.0077 & 0.0282 & 0.5043 & 5.6282 & 0.0308 & 0.4272 & 0.4272 & 0.0002 \\
\hline 502 & 0.9778 & 0.0077 & 0.0255 & 0.2878 & 4.6543 & 0.0322 & 0.5239 & 0.5239 & 0.0098 \\
\hline 503 & 0.9872 & 0 & 0 & 0.2909 & 4.5544 & 0.0411 & 1.4523 & 1.4523 & 0.0087 \\
\hline 504 & 1.1329 & 0 & 0 & 0.3707 & 0 & 0.1128 & 2.4181 & 2.4181 & 0 \\
\hline 505 & 1.9178 & 0 & 0 & 0.3812 & 1.3243 & 0.1724 & 4.9207 & 4.9207 & 0 \\
\hline$\ldots$ & $\ldots$ & $\ldots$ & $\ldots$ & $\ldots$ & $\ldots$ & $\ldots$ & $\ldots$ & $\ldots$ & $\ldots$ \\
\hline$\ldots$ & $\ldots$ & $\ldots$ & $\ldots$ & $\ldots$ & $\ldots$ & $\ldots$ & $\ldots$ & $\ldots$ & $\ldots$ \\
\hline 1102 & 0 & 0.0331 & 0.0056 & 0.9297 & 3.9502 & 0 & 0.2853 & 1.8033 & 1.1355 \\
\hline 1103 & 1.5077 & 0.0626 & 0.0332 & 1.1017 & 2.8221 & 0 & 0 & 0 & 1.4133 \\
\hline 1104 & 0 & 0.0435 & 0.5821 & 0.1939 & 4.5585 & 0 & 0 & 0 & 1.1672 \\
\hline 1105 & 0 & 0 & 1.52970 & 0.1922 & 3.2418 & 0.7273 & 0.2726 & 0.2726 & 0 \\
\hline 1106 & 4.4476 & 0.323 & 0.5112 & 3.5487 & 3.8113 & 1.0179 & 0.3296 & 0.3296 & 0 \\
\hline 1107 & 2.4312 & 0.0011 & 0.0435 & 0.2001 & 2.6026 & 0.9354 & 0 & 0 & 1.7245 \\
\hline 1108 & 2.5079 & 0.1232 & 0.4542 & 0.3781 & 0 & 0.8825 & 0.1878 & 0.1878 & 0 \\
\hline 1109 & 1.7828 & 0.0431 & 0.3210 & 0.4024 & 0 & 0.8812 & 0 & 0 & 1.3191 \\
\hline 1110 & 1.2706 & 0.0056 & 1.1123 & 0.3252 & 0 & 0.8078 & 0 & 0 & 1.0233 \\
\hline
\end{tabular}

cointegration between the two energy commodities and other factors such as recurrent exogeneous factors are presented. Villar and Joutz [10] list some economic factors linking natural gas and crude oil prices, while testing for market integration in the United Kingdom during the time when natural gas was deregulated. Asche et al. [6] have integrated the prices of the energy commodities: natural gas, electricity, and crude oil.

Most of this work is centered around the relationship between prices of energy commodities. We are interested in an interdependence of certain energy commodities. Moreover, a nonlinear multivariate interconnected stochastic model of energy commodities and sources with and without external random intervention processes is developed. Also, parameter and state estimation problems of continuous time nonlinear stochastic dynamic process are motivated to initiate an alternative innovative approach. This led to the introduction of the concept of statistic processes, namely, local sample mean and sample variance which further led to the development of an interconnected discrete-time dynamic system of local statistic processes and its mathematical model discussed in Otunuga et al. [16, 17]. This paved the way for extending the LLGMM [16] to a multivariate local lagged adapted generalized method of moments case. The parameters in the multivariate model are estimated using the LLGMM method. These estimated parameters help in 


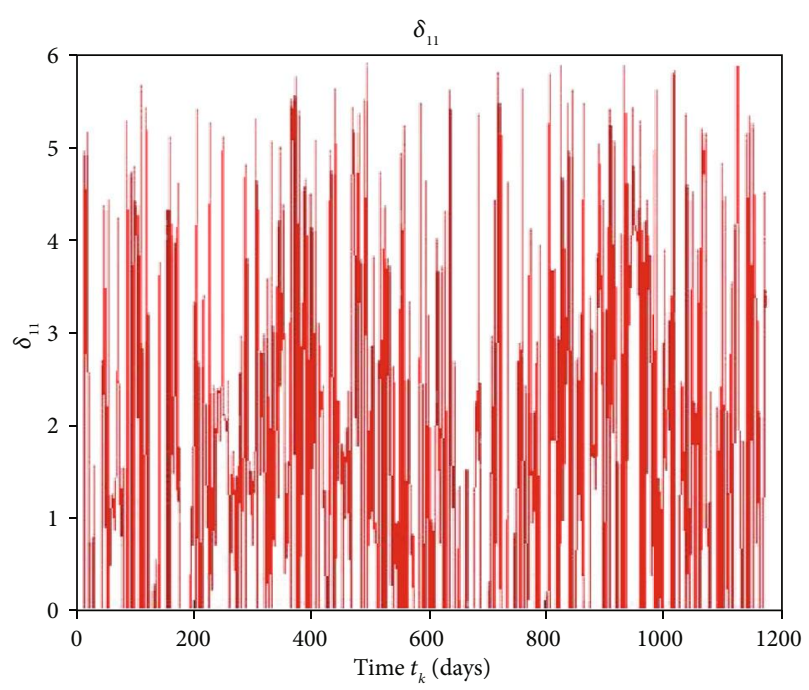

(a)

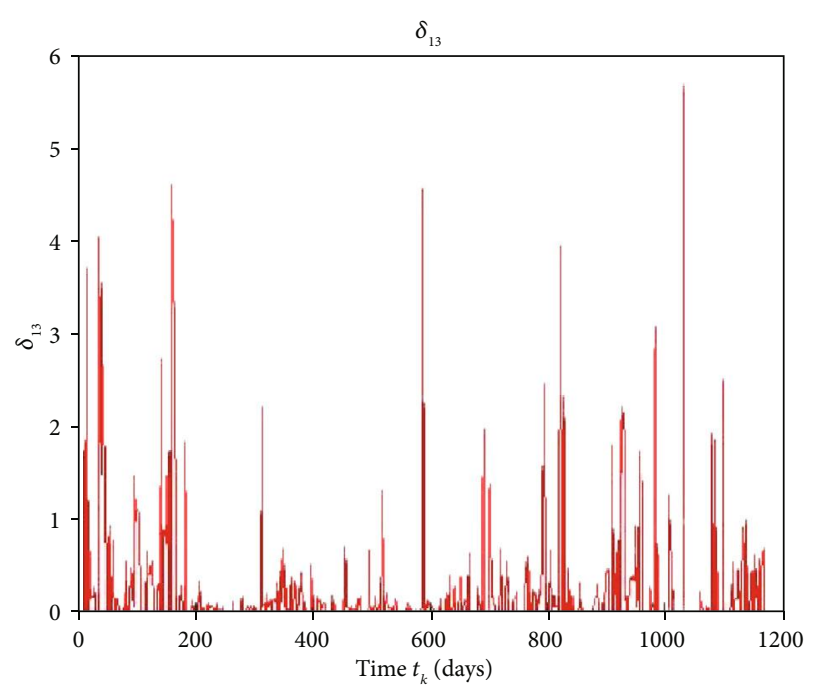

(c)

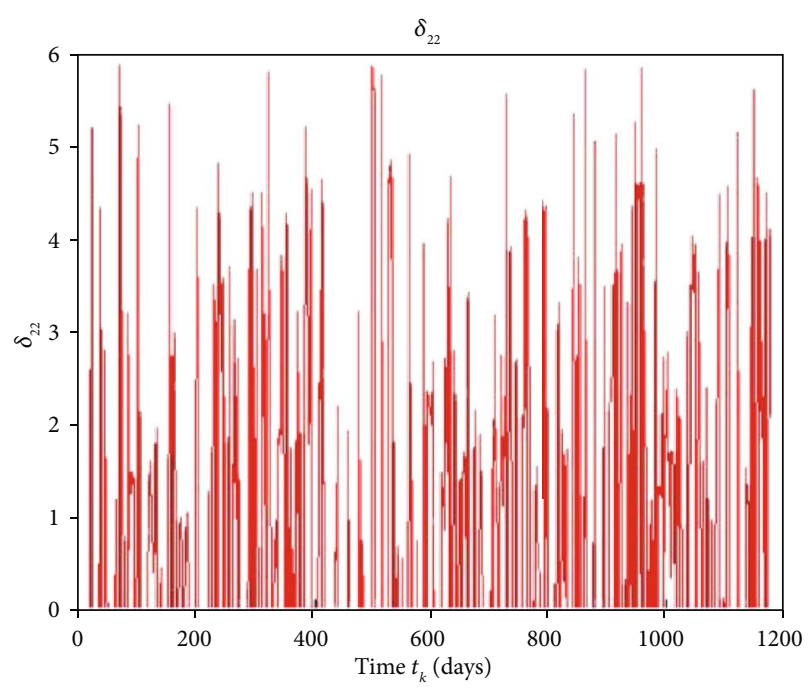

(e)

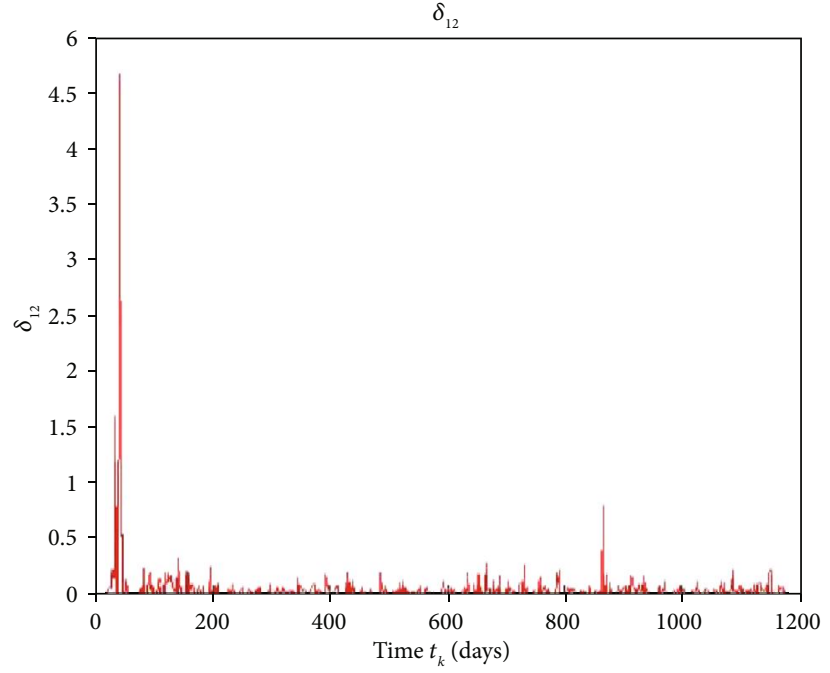

(b)

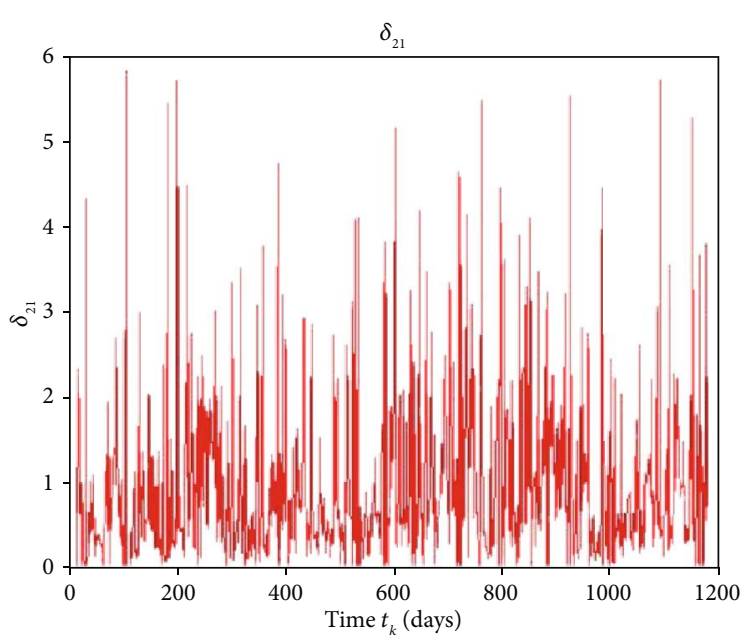

(d)

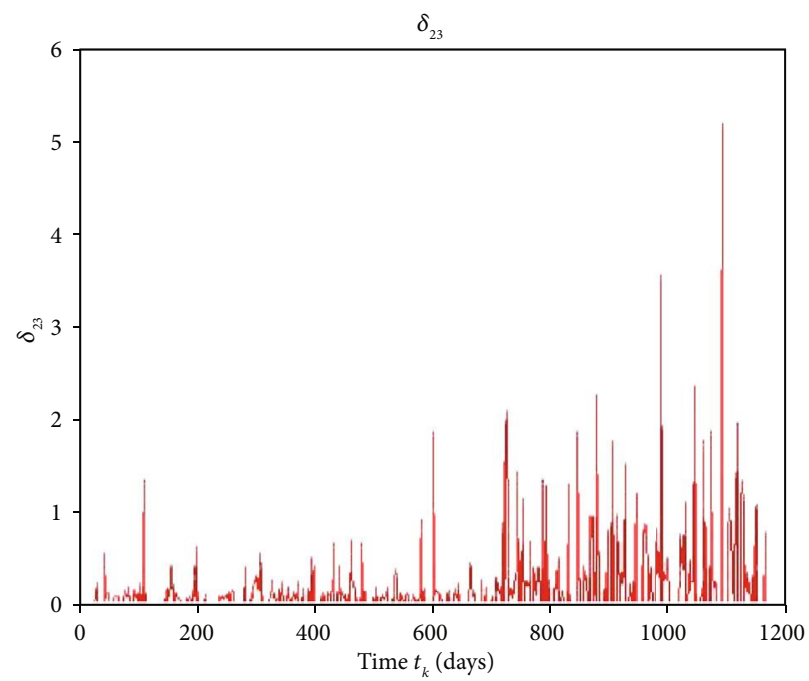

(f)

Figure 3: Continued. 


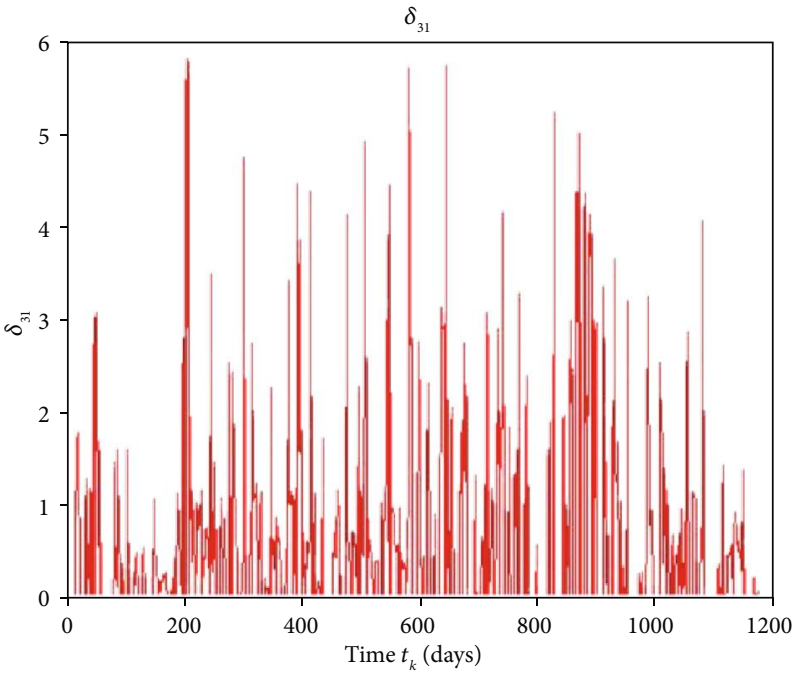

(g)

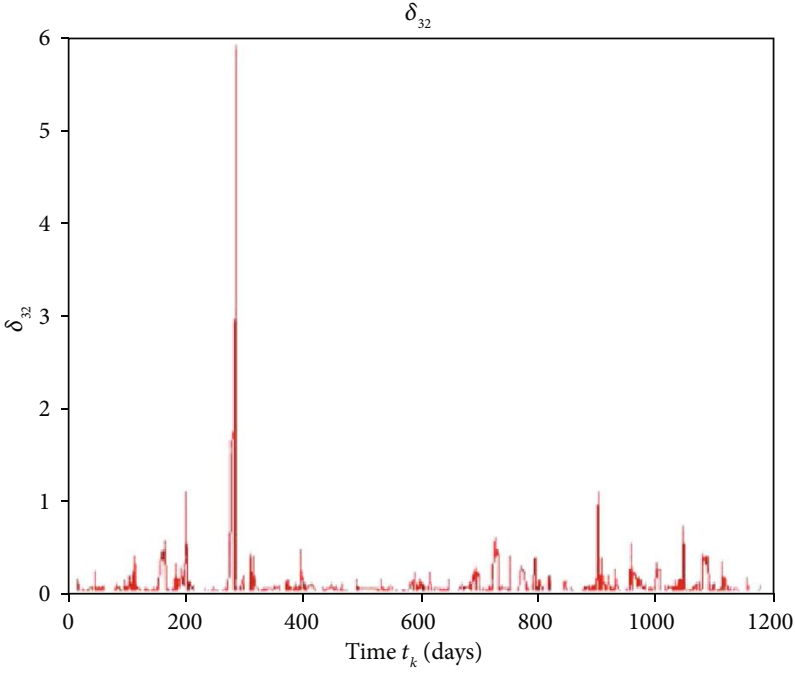

(h)

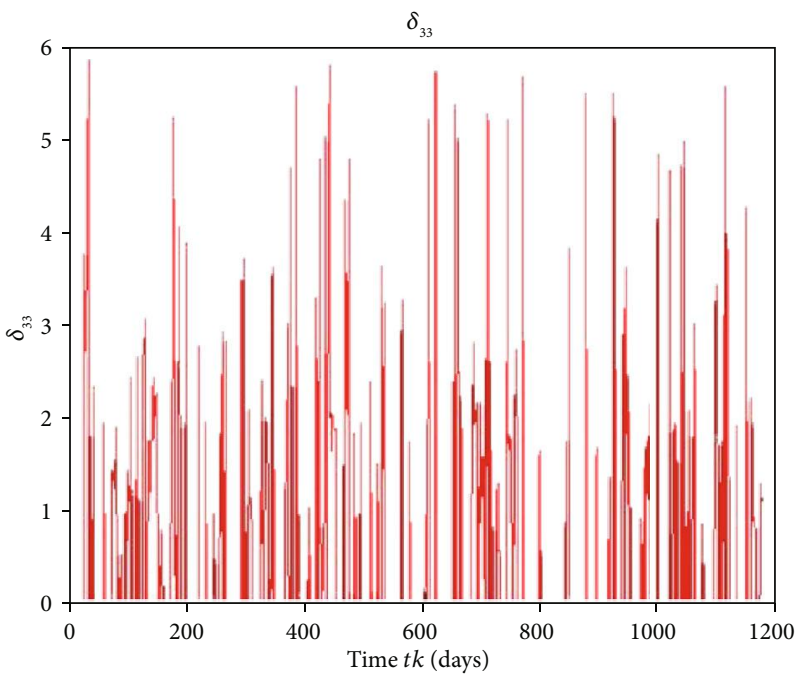

(i)

FIGURE 3: The graph of interaction coefficients $\delta_{1,1}\left(\widehat{m}_{k}, k\right), \delta_{1,2}\left(\widehat{m}_{k}, k\right), \delta_{1,3}\left(\widehat{m}_{k}, k\right), \delta_{2,1}\left(\widehat{m}_{k}, k\right), \delta_{2,2}\left(\widehat{m}_{k}, k\right), \delta_{2,3}\left(\widehat{m}_{k}, k\right), \delta_{3,1}\left(\widehat{m}_{k}, k\right), \delta_{3,2}\left(\widehat{m}_{k}, k\right)$, and $\delta_{3,3}\left(\widehat{m}_{k}, k\right)$.

analyzing the short- and long-term relationships between the energy commodities. It has been shown in Appendices A, B, $\mathrm{C}$, and D of the work of Otunuga et al. [16] that the LLGMM parameter estimation scheme performs better than existing nonparametric statistical methods. A performance comparison (with appropriate statistical indices) of the LLGMM method with existing orthogonality condition based on generalized method of moments (OCBGMM) and aggregated generalized method of moments (AGMM) methods using the energy commodity stochastic model is discussed in their work. The method is applied to estimate time-varying parameter estimates in a stochastic differential equation governing energy commodities, stock price processes, and transmission of infectious diseases in the work of Otunuga et al. [16], Otunuga [18], and Mummert and Otunuga [19], respectively. Algorithm for implementing the LLGMM approach is presented in Otunuga et al. [17]. In this work, the usefulness of this approach is further illustrated by applying the technique to study the relationship between three energy commodity data sets: Henry Hub natural gas, crude oil, and coal data sets for state and parameter estimation problems. Interested readers are directed to the work of Otunuga et al. $[16-18,20]$ to read more about the LLGMM parameter estimation procedure. Moreover, the forecasting and confidence interval problems are also investigated.

The organization of this paper is as follows.

In Section 2, we derive a multivariate stochastic dynamical model for energy commodities. In Section 3, the multivariate model derived in Section 2 is validated. Due to random intervention, we introduce interventions described by a continuous jump process in our model in Section 4. In Section 5, we discuss the discrete-time dynamic model for sample mean and covariance processes with jump using the work of Otunuga et al. in $[16,17]$. In Section 6, we discuss about 


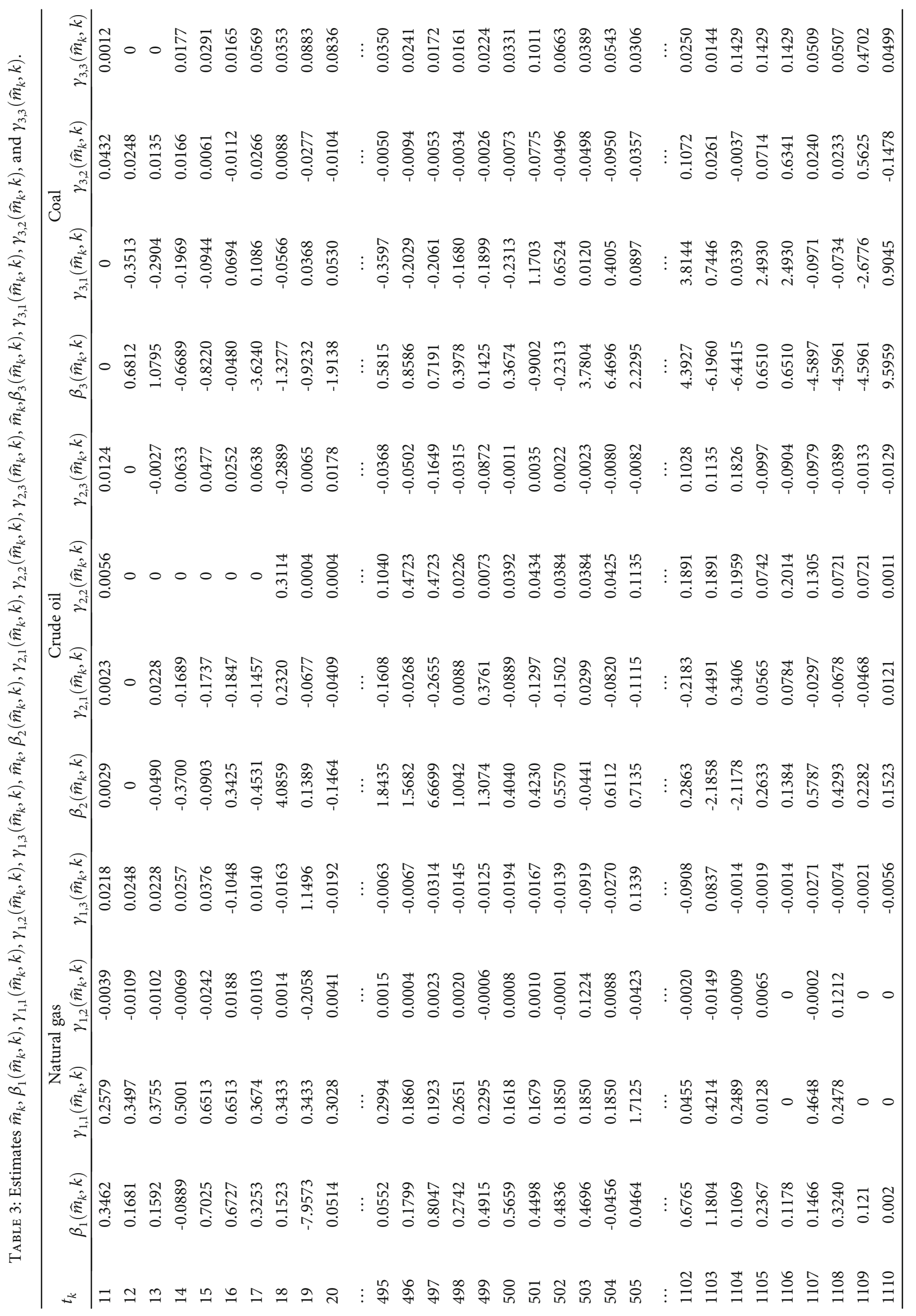




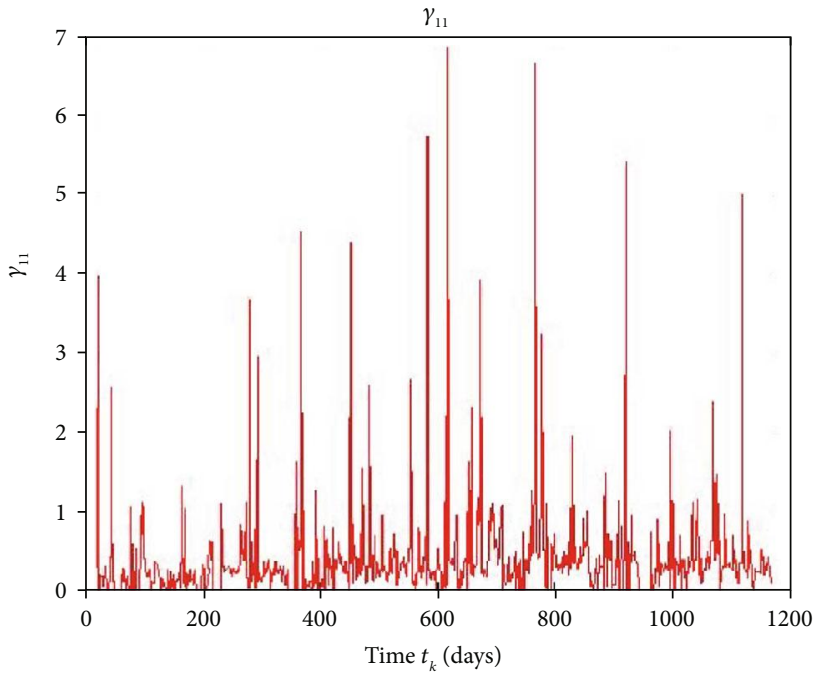

(a)

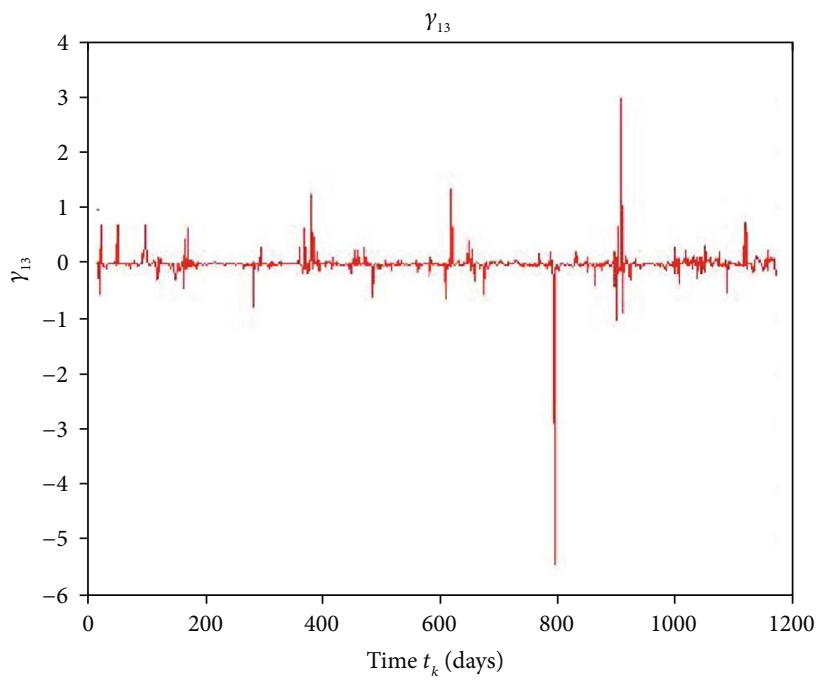

(c)

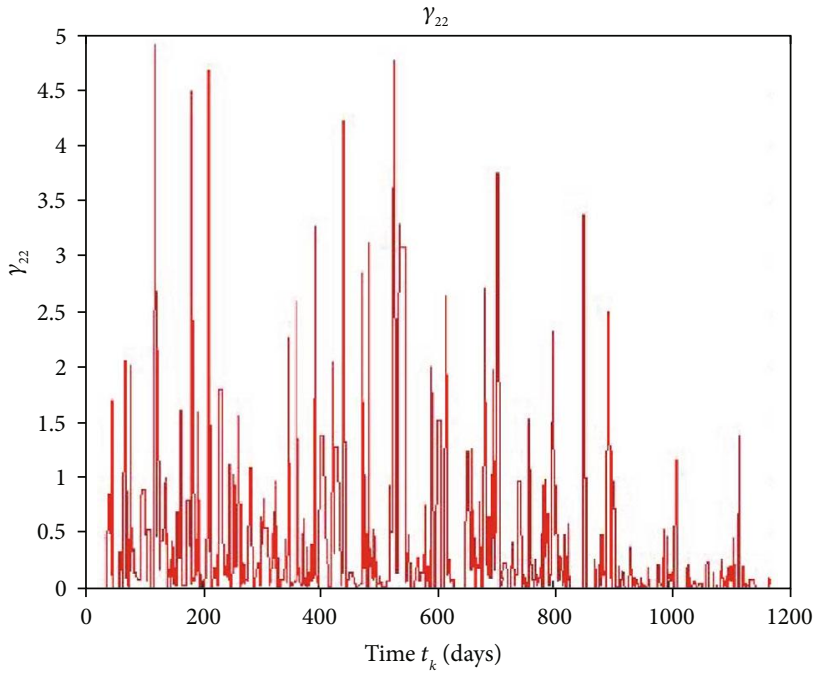

(e)

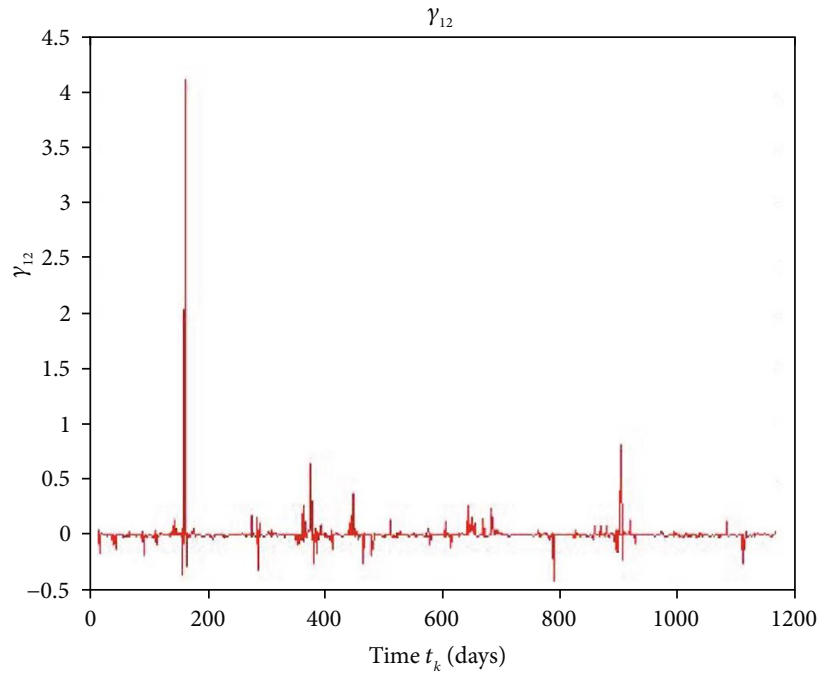

(b)

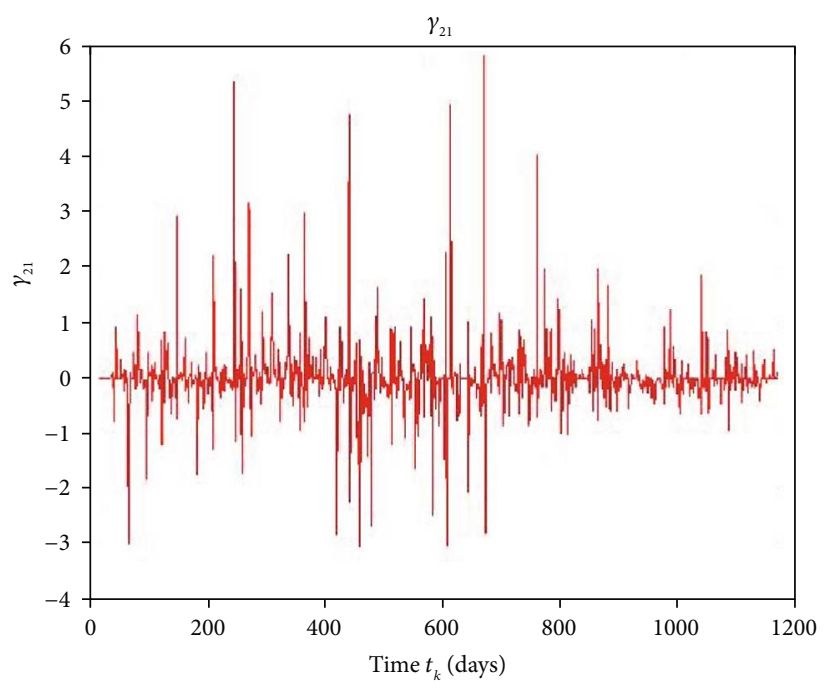

(d)

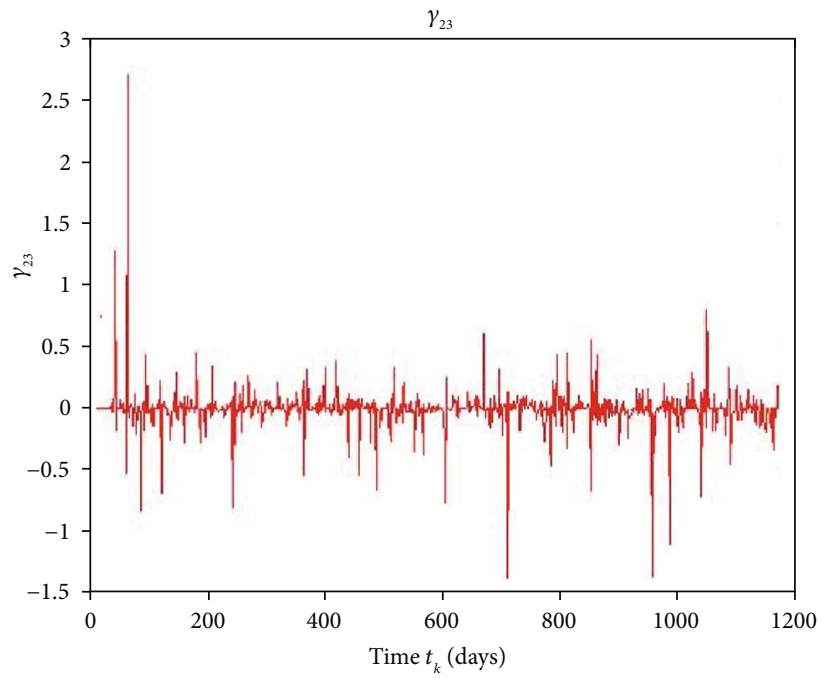

(f)

Figure 4: Continued. 


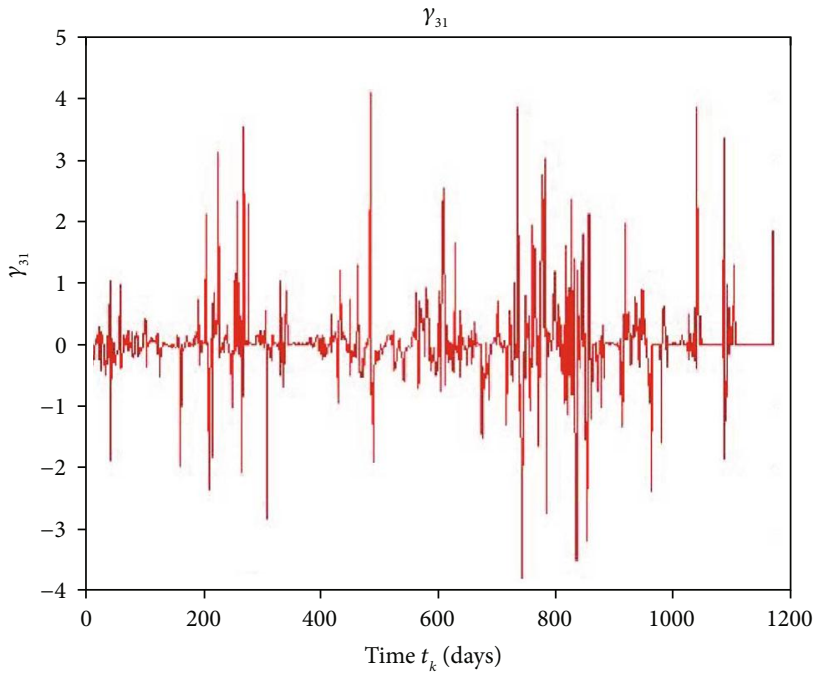

(g)

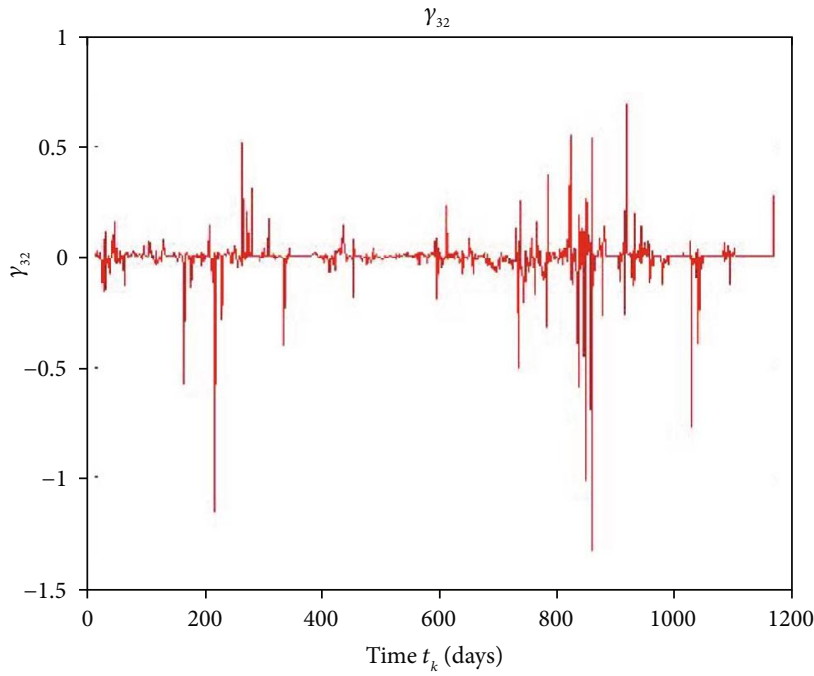

(h)

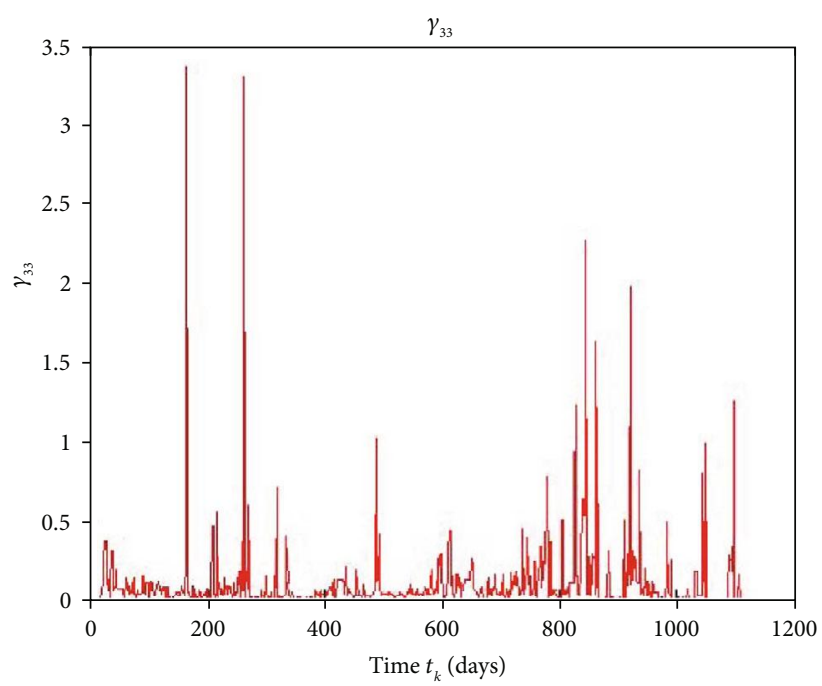

(i)

FIGURE 4: The graph of interaction coefficients $\gamma_{1,1}\left(\widehat{m}_{k}, k\right), \gamma_{1,2}\left(\widehat{m}_{k}, k\right), \gamma_{1,3}\left(\widehat{m}_{k}, k\right), \gamma_{2,1}\left(\hat{m}_{k}, k\right), \gamma_{2,2}\left(\widehat{m}_{k}, k\right), \gamma_{2,3}\left(\hat{m}_{k}, k\right), \gamma_{3,1}\left(\widehat{m}_{k}, k\right), \gamma_{3,2}\left(\widehat{m}_{k}, k\right)$, and $\gamma_{3,3}\left(\widehat{m}_{k}, k\right)$.

parametric estimation techniques. We construct an observation system from a nonlinear stochastic functional differential equation. In Section 7, using the method of moments [21], in the context of lagged adaptive expectation process [22], we briefly outline a procedure to estimate the state parameters for the dynamical model with jump and the model without jump locally. Moreover, the usefulness of computational algorithm is illustrated by applying the procedure to test for the relationship between Henry Hub natural gas, crude oil, and coal for the state and parameter estimation problems. In Section 8, the forecasting and confidence interval problems are also addressed.

\section{Model Derivation}

Let $p=\left[p_{1}, p_{2}, \cdots, p_{n}\right]^{T}$ be a vector of $n$ energy commodity prices considered to have long-run or short-run relationship with each other, with $p_{j}(t)$ being the price of the $j$ th energy commodity at time $t$. The economic principles of demand and supply processes suggest that the price of an energy commodity will remain within a given finite expected lower and upper bounds. We define $u_{j} \in \mathfrak{R}_{+}=(0, \infty)$ and $l_{j} \geq 0$ as the expected upper and lower limits of the $j$ th energy commodity spot prices, respectively. In the absence of interactions between the energy commodities $p_{j}, j \in I(1, n)$, where

$$
I(a, b)=\{z \in \mathbb{Z}: a \leq z \leq b\},
$$

the market potential for the $j$ th commodity per unit of time at time $t$ can be characterized by $\left(u_{j}-p_{j}\right)\left(l_{j}+p_{j}\right)$. This simple idea leads to the following economic principle regarding the 


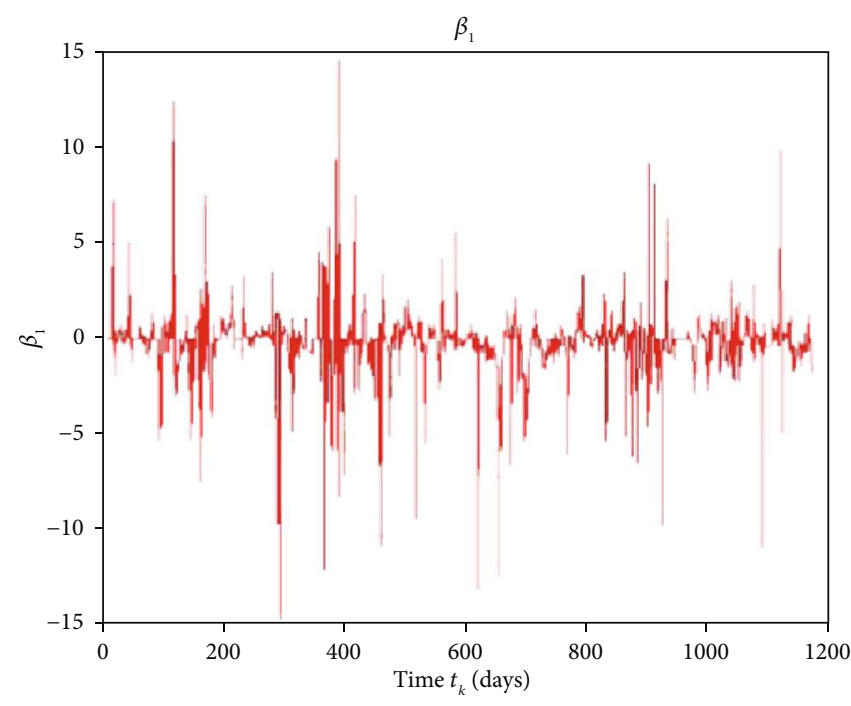

(a)

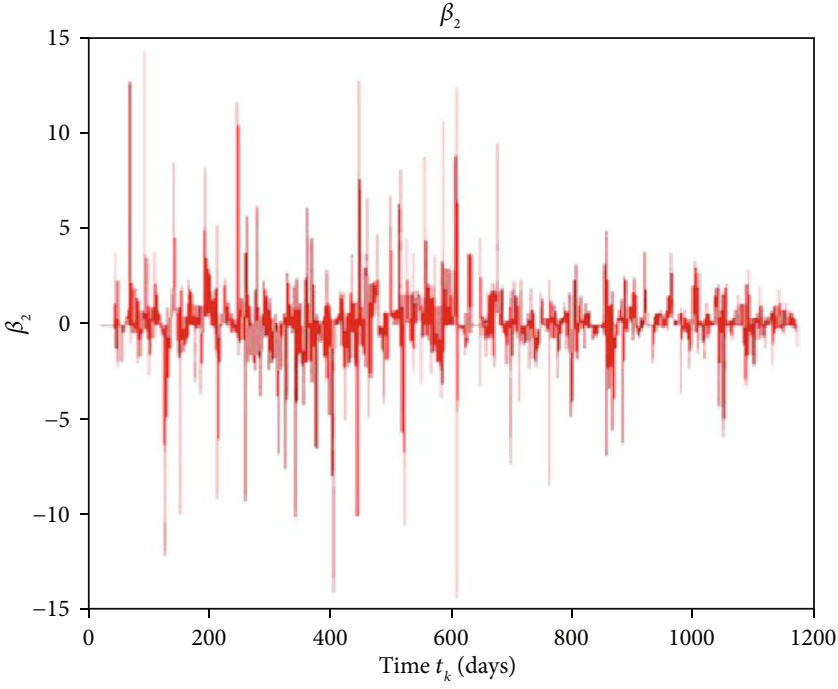

(b)

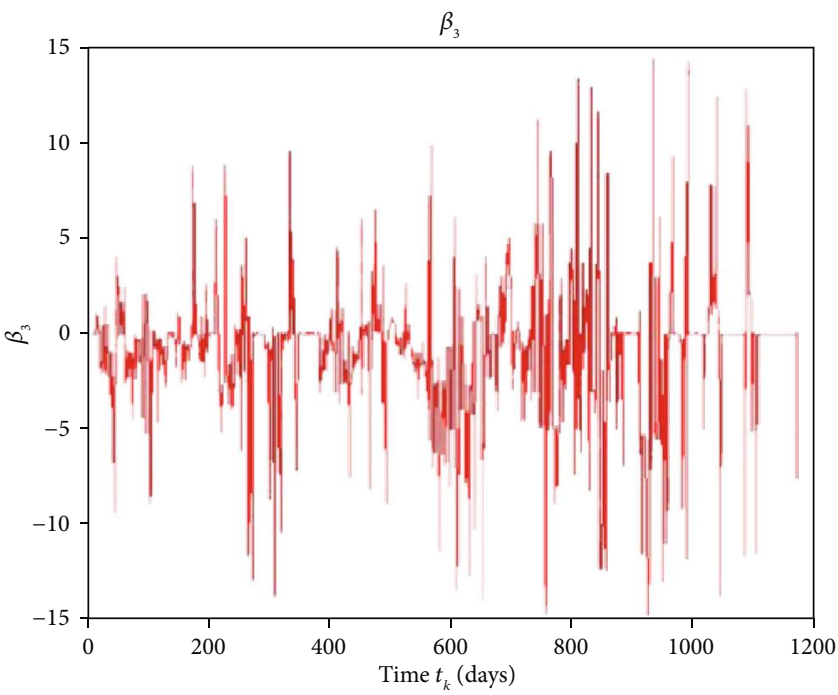

(c)

FIGURE 5: The graph of $\beta_{1}\left(\widehat{m}_{k}, k\right), \beta_{2}\left(\widehat{m}_{k}, k\right)$, and $\beta_{3}\left(\widehat{m}_{k}, k\right)$ for natural gas, crude oil, and coal, respectively.

dynamic of the price $p_{j}$ of the jth energy commodity. The change in spot price of the $j$ th energy commodity $\Delta p_{j}(t)=$ $p_{j}(t+\Delta t)-p_{j}(t)$ over the interval of length $|\Delta t|$ is directly proportional to the market potential price, that is,

$$
\Delta p_{j}(t) \propto\left(u_{j}-p_{j}\right)\left(l_{j}+p_{j}\right) \Delta t
$$

This implies that

$$
d p_{j}=\alpha_{j}\left(u_{j}-p_{j}\right)\left(l_{j}+p_{j}\right) d t
$$

for some constant $\alpha_{j}$. From this deterministic mathematical model, if $\alpha_{j}>0$, we note that as the price falls below the expected price $u_{j}$, the price of the $j$ th commodity rises, and as the price lies above $u_{j}$, there is a tendency for the price to fall. Similar argument follows if $\alpha_{j}<0$. Hence $u_{j}$ is the equilibrium state of (3).

In a real world situation, the expected upper price limit $u_{j}$ of the $j$ th commodity is not a constant parameter. It varies with time, and moreover it is subjected to random environmental perturbations. Therefore, we consider

$$
u_{j}=y_{j}+\xi_{j}
$$

where $\xi_{j}$ is a white noise process that characterizes the measure of random fluctuation of the upper price limit of the $j$ th commodity; here $y_{j}$ stands for the mean of the energy spot 
TABLE 4: Estimates $\sigma_{1,1}\left(\widehat{m}_{k}, k\right), \sigma_{1,2}\left(\widehat{m}_{k}, k\right), \sigma_{1,3}\left(\widehat{m}_{k}, k\right), \sigma_{2,1}\left(\widehat{m}_{k}, k\right), \sigma_{2,2}\left(\widehat{m}_{k}, k\right), \sigma_{2,3}\left(\widehat{m}_{k}, k\right), \sigma_{3,1}\left(\widehat{m}_{k}, k\right), \sigma_{3,2}\left(\widehat{m}_{k}, k\right)$, and $\sigma_{3,3}\left(\widehat{m}_{k}, k\right)$.

\begin{tabular}{|c|c|c|c|c|c|c|c|c|c|}
\hline$t_{k}$ & $\sigma_{1,1}\left(\widehat{m}_{k}, k\right)$ & $\begin{array}{c}\text { Natural gas } \\
\sigma_{1,2}\left(\widehat{m}_{k}, k\right)\end{array}$ & $\sigma_{1,3}\left(\widehat{m}_{k}, k\right)$ & $\sigma_{2,1}\left(\widehat{m}_{k}, k\right)$ & $\begin{array}{c}\text { Crude oil } \\
\sigma_{2,2}\left(\widehat{m}_{k}, k\right)\end{array}$ & $\sigma_{2,3}\left(\widehat{m}_{k}, k\right)$ & $\sigma_{3,1}\left(\widehat{m}_{k}, k\right)$ & $\begin{array}{c}\text { Coal } \\
\sigma_{3,2}\left(\widehat{m}_{k}, k\right)\end{array}$ & $\sigma_{3,3}\left(\widehat{m}_{k}, k\right)$ \\
\hline 11 & 0 & 0 & 0 & 0 & 0 & 0 & 0 & 0 & 0 \\
\hline 12 & 0.0485 & 0.0004 & 0.0032 & 0.2734 & 0.0166 & 0 & 0 & 0 & 0.0000 \\
\hline 13 & 0 & 0 & 0 & 0 & 0 & 0 & 0 & 0 & 0 \\
\hline 14 & 0.2120 & 0.1386 & 0.0133 & 1.2573 & 0.4773 & 0.1195 & 0 & 0.0665 & 0.0086 \\
\hline 15 & 0.4246 & 0.1318 & 0.0021 & 2.1081 & 0.4894 & 0.1211 & 0 & 0.6107 & 0.0696 \\
\hline 16 & 0.5538 & 0.0778 & 0.1501 & 0 & 0.2524 & 0.0811 & 0.0651 & 0.4251 & 0.0635 \\
\hline 17 & 1.1121 & 0.0469 & 0.2230 & 0 & 0.1848 & 0.2463 & 0 & 0.4458 & 0.0478 \\
\hline 18 & 1.5347 & 0.0180 & 0.2178 & 0 & 0.1877 & 0.1602 & 0.5681 & 0.0592 & 0.0115 \\
\hline 19 & 1.1315 & 0.0619 & 0.2221 & 0 & 0.2673 & 0.2465 & 0.4999 & 0.0569 & 0.0127 \\
\hline 20 & 2.0845 & 0.0536 & 0.1866 & 0 & 0.1700 & 0.0781 & 0.3789 & 0.3174 & 0.0046 \\
\hline$\ldots$ & $\ldots$ & $\ldots$ & $\ldots$ & $\ldots$ & $\ldots$ & $\ldots$ & $\ldots$ & $\ldots$ & $\ldots$ \\
\hline 495 & 0 & 0.0036 & 0.0406 & 0.2286 & 0.0600 & 0.0172 & 0 & 0.9387 & 0.0182 \\
\hline 496 & 0.1588 & 0.0035 & 0.0107 & 1.4847 & 0.3163 & 0.0102 & 0 & 0 & 0.0016 \\
\hline 497 & 0.1551 & 0.0009 & 0.0065 & 0 & 0.1453 & 0 & 0.7777 & 0 & 0.0033 \\
\hline 498 & 0.1576 & 0.0011 & 0.0073 & 0 & 0.1679 & 0 & 0.5334 & 0 & 0.0060 \\
\hline 499 & 0.1197 & 0.0006 & 0.0059 & 1.9414 & 0.2391 & 0.0172 & 0.4405 & 0.1432 & 0.0097 \\
\hline 500 & 0.3600 & 0.0001 & 0.0049 & 1.9554 & 0.3960 & 0.0079 & 0.6331 & 0.1410 & 0.0093 \\
\hline 501 & 0.0514 & 0.0033 & 0.0049 & 2.0436 & 0.3499 & 0.0111 & 0.7690 & 0.1376 & 0.0089 \\
\hline 502 & 0.2503 & 0.0034 & 0.0042 & 2.0837 & 0.1744 & 0.0132 & 0.6198 & 0.1274 & 0.0066 \\
\hline 503 & 0.1195 & 0.0147 & 0.0165 & 0 & 0.4283 & 0.0060 & 1.1613 & 0.1530 & 0.0049 \\
\hline 504 & 0.0974 & 0.0144 & 0.0027 & 0 & 0.2241 & 0.0048 & 0.4778 & 0.0574 & 0.0043 \\
\hline 505 & 0.1422 & 0.0060 & 0.0131 & 0 & 0.2023 & 0.0054 & 0.5604 & 0.0669 & 0.0004 \\
\hline$\ldots$ & $\ldots$ & $\ldots$ & $\ldots$ & $\ldots$ & $\ldots$ & $\ldots$ & $\ldots$ & $\ldots$ & $\ldots$ \\
\hline 1102 & 0.1898 & 0.0016 & 0.0413 & 0.8313 & 0.0767 & 0.0381 & 0.6875 & 0 & 0.1451 \\
\hline 1103 & 0.2094 & 0.0015 & 0.0352 & 0.8262 & 0.0673 & 0.0451 & 0.7298 & 0.2808 & 0.0147 \\
\hline 1104 & 0.1711 & 0.0011 & 0.0040 & 0.6648 & 0.0915 & 0.0462 & 0.5563 & 0.1831 & 0.0105 \\
\hline 1105 & 0.1816 & 0.0012 & 0.0116 & 0.6658 & 0.1049 & 0.0371 & 0.6591 & 0.2874 & 0.0057 \\
\hline 1106 & 0.1191 & 0.0011 & 0.0116 & 0.6260 & 0.1155 & 0.0393 & 0 & 0.0196 & 0.0060 \\
\hline 1107 & 0.0417 & 0.0012 & 0.0041 & 0.4992 & 0.0781 & 0.0382 & 0 & 0 & 0.0065 \\
\hline 1108 & 0.1058 & 0.0033 & 0.0045 & 0.0019 & 0.0589 & 0.0421 & 0 & 0 & 0.0018 \\
\hline 1109 & 0.1740 & 0.0021 & 0 & 0 & 0.0446 & 0.0316 & 2.1187 & 0 & 0.4511 \\
\hline 1110 & 0.2912 & 0.0021 & 0.0163 & 0.0385 & 0.0342 & 0.0037 & 0 & 1.1563 & 0.0257 \\
\hline
\end{tabular}

price process of the $j$ th commodity at time $t$. It is further assumed that $y_{j}$ is governed by a similar dynamic forces described in (3), that is,

$$
d y_{j}=\mu_{j}\left(u_{j}-y_{\mathrm{j}}\right)\left(v_{j}+y_{j}\right) d t
$$

where $\mu_{j} \in \Re_{+}$is defined as the mean reversion rate of the mean of the $j$ th commodity. By following the argument used in (4), we incorporate the effects of random environmental perturbations into the lower limit $v_{j}$ of the mean of the $j$ th commodity:

$$
v_{j}=v_{j}+e_{j},
$$

where $v_{j} \geq 0$, and $e_{j}$ is a white noise process describing the measure of random influence on the mean price of the $j$ th commodity.

Substituting expressions in (4) and (6) into (3) and (5), respectively, we obtain

$$
\left\{\begin{array}{l}
d y_{j}=\mu_{j}\left(u_{j}-y_{j}\right)\left(v_{j}+y_{j}\right) d t+\mu_{j}\left(u_{j}-y_{j}\right) e_{j}(t) d t \\
d p_{j}=\alpha_{j}\left(y_{j}-p_{j}\right)\left(l_{j}+p_{j}\right) d t+\alpha_{j}\left(l_{j}+p_{j}\right) \xi_{j}(t) d t .
\end{array}\right.
$$

In the absence of interactions and using (7), the system of stochastic model for isolated expected spot and spot prices 


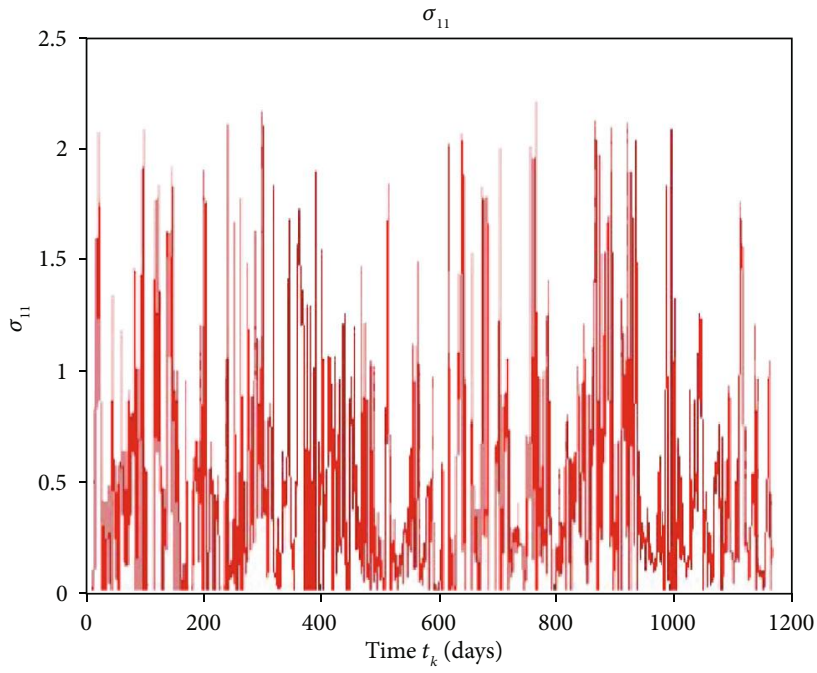

(a)

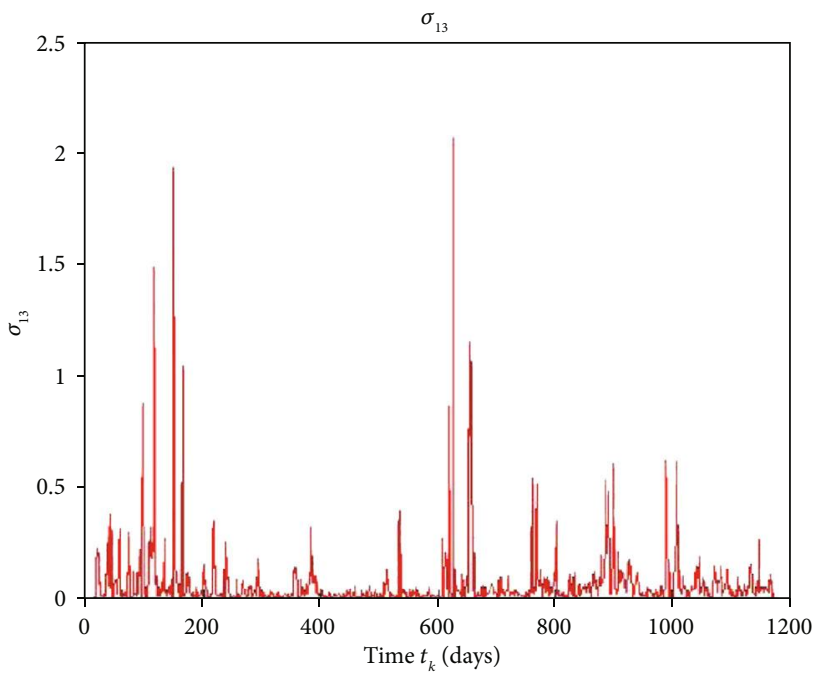

(c)

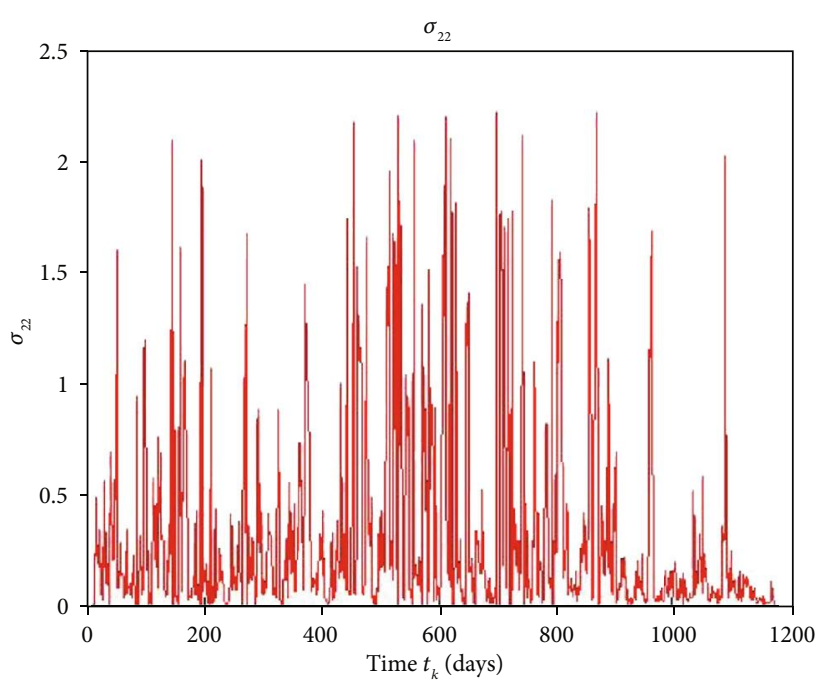

(e)

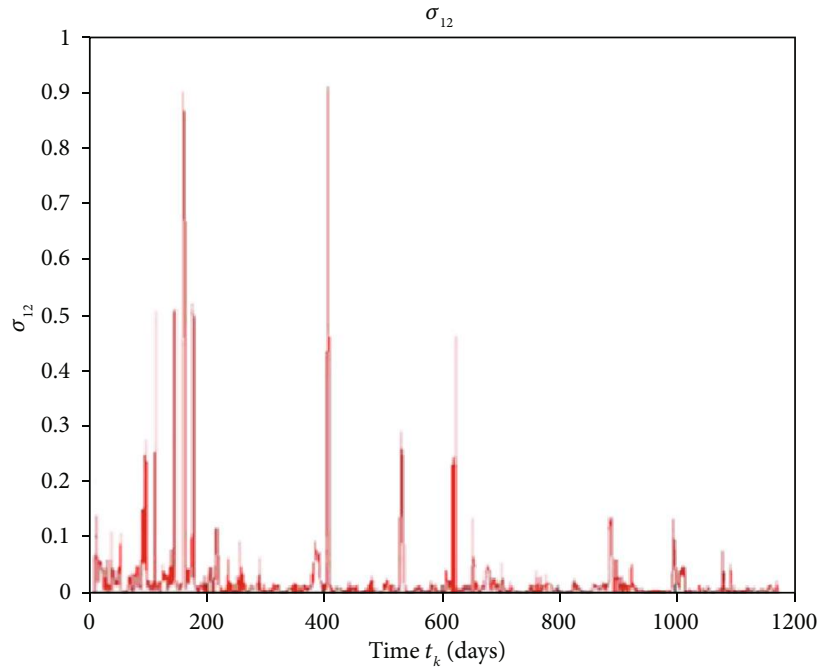

(b)

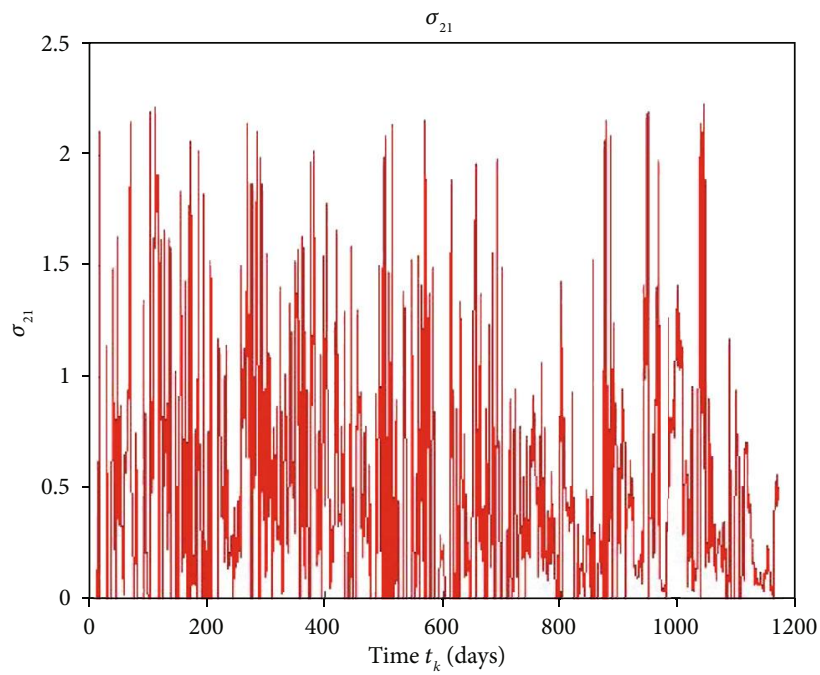

(d)

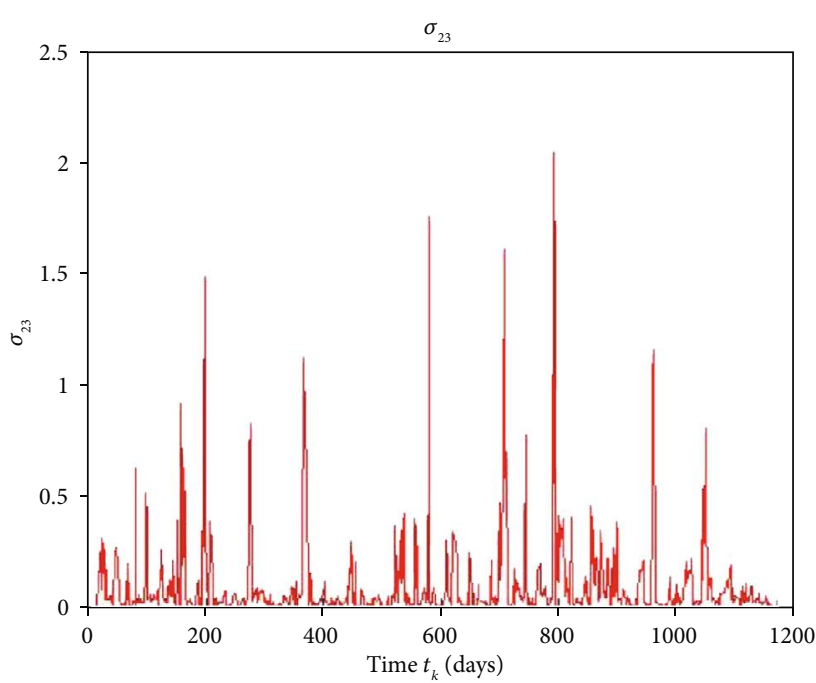

(f)

Figure 6: Continued. 


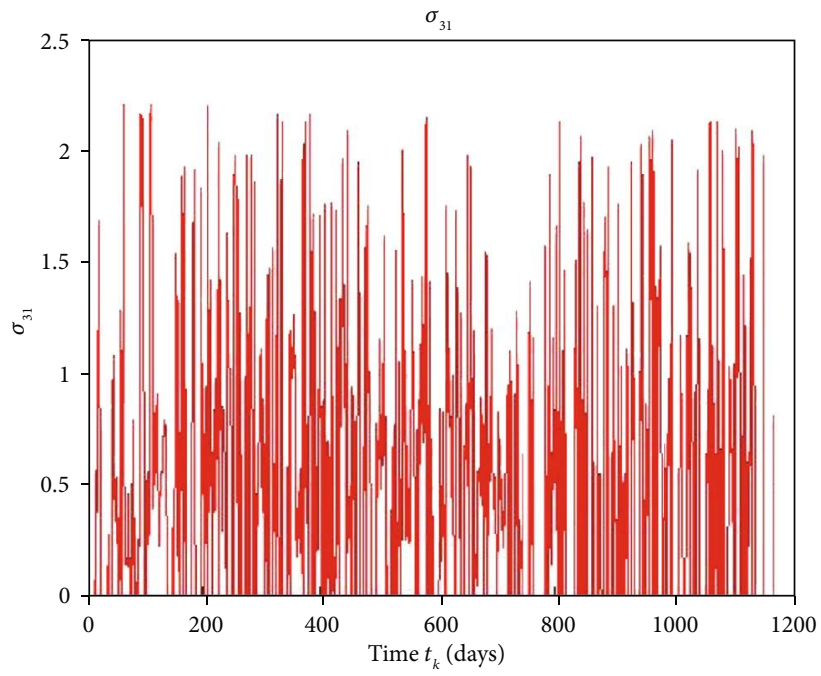

(g)

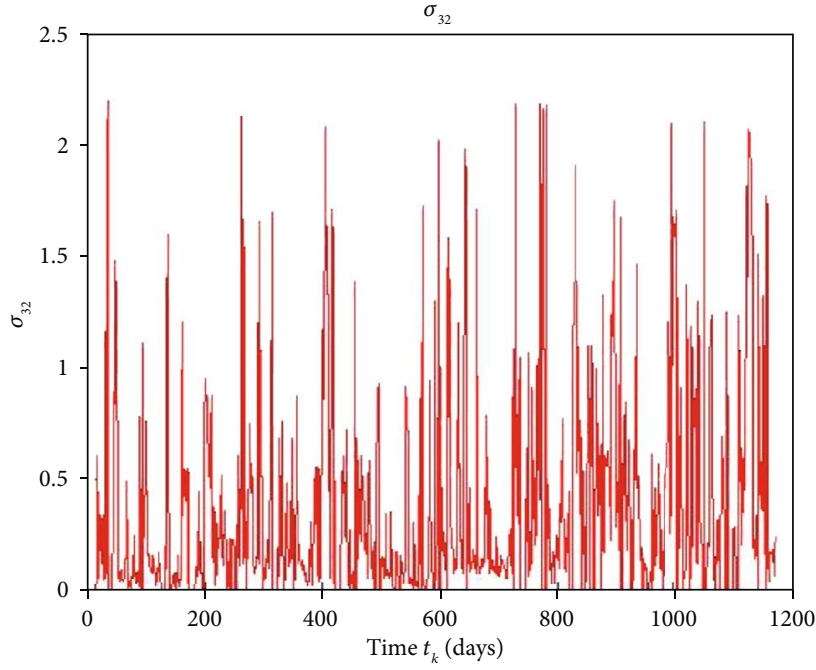

(h)

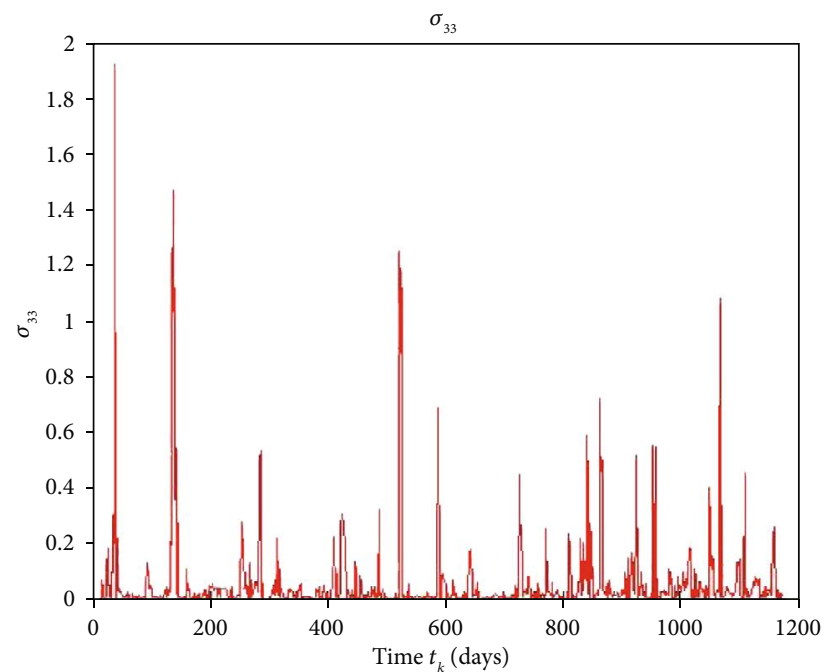

(i)

FIGURE 6: The graph of $\sigma_{1,1}\left(\widehat{m}_{k}, k\right), \sigma_{1,2}\left(\widehat{m}_{k}, k\right), \sigma_{1,3}\left(\widehat{m}_{k}, k\right), \sigma_{2,1}\left(\widehat{m}_{k}, k\right), \sigma_{2,2}\left(\widehat{m}_{k}, k\right), \sigma_{2,3}\left(\widehat{m}_{k}, k\right), \sigma_{3,1}\left(\widehat{m}_{k}, k\right), \sigma_{3,2}\left(\widehat{m}_{k}, k\right)$, and $\sigma_{1,1}\left(\widehat{m}_{k}, k\right)$ for natural gas, crude oil, and coal, respectively.

processes are described by the following nonlinear system of stochastic differential equations:

$$
\left\{\begin{array}{l}
d y_{j}=\mu_{j}\left(u_{j}-y_{j}\right)\left(v_{j}+y_{j}\right) d t+\delta_{j, j}\left(u_{j}-y_{j}\right) d W_{j, j}(t), \quad y_{j}\left(t_{0}\right)=y_{j 0}, \\
d p_{j}=\alpha_{j}\left(y_{j}-p_{j}\right)\left(l_{j}+p_{j}\right) d t+\sigma_{j, j}\left(l_{j}+p_{j}\right) d Z_{j, j}(t), \quad p_{j}\left(t_{0}\right)=p_{j 0}, j \in I(1, n),
\end{array}\right.
$$

where

$$
\left\{\begin{array}{l}
\mu_{j} e_{j}(t) d t=\delta_{j, j} d W_{j, j}(t) \\
\alpha_{j} \xi_{j}(t) d t=\sigma_{j, j} d Z_{j, j}(t)
\end{array}\right.
$$

and $\delta_{j, j}, \sigma_{i, j}$ are nonnegative for $j \in I(1, n)$.
In the presence of interactions, for each $j \in I(1, n)$, we consider both deterministic and stochastic interaction functions. For each $j \in I(1, n)$, we define the $j$ th aggregate interaction functions $\mathbf{g}_{j}:\left[t_{0}, \infty\right) \times \mathfrak{R}^{n} \longrightarrow \mathfrak{R}$ and $\mathbf{h}_{j}:\left[t_{0}\right.$, $\infty) \times \mathfrak{R}^{n} \longrightarrow \mathfrak{R}$ for the $j$ th mean energy spot price process $y_{j}(t)$ and the energy spot price process $\mathbf{p}_{j}(t)$ in energy commodity market network system, respectively. Moreover, 
TABLE 5: Real and simulated estimates (without jump) for natural gas, crude oil, and coal.

\begin{tabular}{|c|c|c|c|c|c|c|}
\hline$t_{k}$ & Real & $\begin{array}{c}\text { Natural gas } \\
\text { Simulated } p_{1}^{s}\left(\widehat{m}_{k}, k\right)\end{array}$ & Real & $\begin{array}{c}\text { Crude oil } \\
\text { Simulated } p_{2}^{s}\left(\widehat{m}_{k}, k\right)\end{array}$ & Real & $\begin{array}{c}\text { Coal } \\
\text { Simulated } p_{3}^{s}\left(\widehat{m}_{k}, k\right)\end{array}$ \\
\hline 11 & 4.0200 & 4.0500 & 58.9900 & 56.5200 & 16.5900 & 16.8000 \\
\hline 12 & 3.9900 & 4.0500 & 59.5200 & 59.3099 & 17.4600 & 16.8635 \\
\hline 13 & 3.7500 & 3.6690 & 61.4500 & 59.3377 & 17.8900 & 17.8086 \\
\hline 14 & 3.7700 & 3.6341 & 60.4900 & 59.4191 & 17.5500 & 17.0859 \\
\hline 15 & 3.4100 & 3.3967 & 61.1500 & 59.6974 & 17.4100 & 17.0859 \\
\hline 16 & 3.3500 & 3.3967 & 62.4800 & 59.6974 & 16.7500 & 17.0859 \\
\hline 17 & 3.4900 & 3.4537 & 63.4100 & 61.2177 & 17.6600 & 19.0677 \\
\hline 18 & 3.5500 & 3.4537 & 65.0900 & 61.4561 & 17.5200 & 16.0578 \\
\hline 19 & 3.9200 & 3.8618 & 66.3100 & 61.6529 & 18.5000 & 19.0677 \\
\hline 20 & 3.8600 & 3.8618 & 68.5900 & 60.9364 & 19.0600 & 19.0677 \\
\hline$\ldots$ & $\ldots$ & $\ldots$ & $\ldots$ & $\ldots$ & $\ldots$ & $\ldots$ \\
\hline 495 & 4.1900 & 4.0368 & 107.1800 & 104.1295 & 32.7600 & 31.3108 \\
\hline 496 & 4.3300 & 4.1868 & 110.8400 & 111.1245 & 33.6500 & 32.7737 \\
\hline 497 & 4.3300 & 4.1025 & 111.7200 & 112.4675 & 33.7100 & 33.4888 \\
\hline 498 & 4.3700 & 4.0964 & 111.6800 & 110.8795 & 34.7500 & 35.5907 \\
\hline 499 & 4.3200 & 4.1042 & 111.7200 & 104.2465 & 34.5400 & 32.9391 \\
\hline 500 & 4.3500 & 4.0548 & 112.3100 & 109.9535 & 34.0400 & 36.2674 \\
\hline 501 & 4.3800 & 4.0548 & 112.3800 & 109.9995 & 33.1000 & 36.2674 \\
\hline 502 & 4.5100 & 4.3249 & 113.3900 & 104.3254 & 33.6700 & 34.8915 \\
\hline 503 & 4.6000 & 4.3555 & 113.0300 & 113.2356 & 33.9400 & 35.0472 \\
\hline 504 & 4.6000 & 4.3491 & 110.6000 & 103.9435 & 33.8300 & 32.8992 \\
\hline 505 & 4.5900 & 4.3609 & 108.7900 & 104.9995 & 32.0200 & 32.8992 \\
\hline$\ldots$ & $\ldots$ & $\ldots$ & $\ldots$ & $\ldots$ & $\ldots$ & $\ldots$ \\
\hline 1102 & 3.7200 & 3.5963 & 108.2300 & 110.5149 & 4.7700 & 2.8861 \\
\hline 1103 & 3.7300 & 3.5963 & 106.2600 & 105.8076 & 5.0100 & 5.6871 \\
\hline 1104 & 3.6800 & 3.4099 & 104.7000 & 105.8076 & 4.9800 & 5.3821 \\
\hline 1105 & 3.6600 & 3.4356 & 103.6200 & 105.8076 & 4.7300 & 4.9221 \\
\hline 1106 & 3.5900 & 3.4636 & 103.2200 & 106.9547 & 4.6800 & 4.2352 \\
\hline 1107 & 3.5200 & 3.2573 & 102.6800 & 105.4047 & 4.6300 & 5.8172 \\
\hline 1108 & 3.4900 & 2.8981 & 103.1000 & 102.4928 & 4.7400 & 6.0376 \\
\hline 1109 & 3.5100 & 2.8981 & 102.8600 & 102.4928 & 4.3300 & 5.1121 \\
\hline 1110 & 3.4800 & 3.0267 & 102.3600 & 102.4928 & 4.1800 & 4.8978 \\
\hline
\end{tabular}

we assume that these functions have the following structural forms:

$$
\left\{\begin{array}{l}
\mathbf{g}_{j}(t, \mathbf{y})=\mathbf{g}_{j}\left(t, k_{j, 1} y_{1}, k_{j, 2} y_{2}, \cdots, k_{j, n} y_{n}\right), \\
\boldsymbol{h}_{j}(t, \mathbf{p})=\boldsymbol{h}_{j}\left(t, \gamma_{j, 1} p_{1}, \gamma_{j, 2} p_{2}, \cdots, \gamma_{j, n} p_{n}\right),
\end{array}\right.
$$

where $k_{j, i}$ and $\gamma_{j, i}$ are elements of $n \times n$ interconnection matrices denoted by $\mathbf{E}_{g}$ and $\mathbf{E}_{h}$, respectively. In (10), $k_{j, i}$ and $\gamma_{j, i}$ represent a degree of interaction of the $j$ th commodity with the $i$ th commodity in the energy commodity market network system.

For the matrix $\mathbf{E}_{g}, k_{j, i}=0$ with fixed $i \in I(1, n)$ if the $i$ th commodity in the energy market network system does not influence the $j$ th commodity. Likewise, for the matrix $\mathbf{E}_{h}$, $\gamma_{j, i}=0$ with fixed $i \in I(1, n)$, if the $j$ th commodity in the energy market network system subcomponent of $\mathbf{p}$ is totally unaffected by the influence of the $i$ th commodity. Finally, we introduce interactions in the diffusion coefficients with respect to the $j$ th commodity of the energy market network system under random environmental perturbations as $\boldsymbol{\psi}_{j}$ : $\left[t_{0}, \infty\right) \times \mathfrak{R}^{n} \longrightarrow \mathfrak{R}^{n}$ and $\boldsymbol{\Lambda}_{j}:\left[t_{0}, \infty\right) \times \mathfrak{R}^{n} \longrightarrow \mathfrak{R}^{n}$ for each $j \in I(1, n)$. The diffusion part is of the form

$$
\left\{\begin{array}{l}
\boldsymbol{\psi}_{j}(t, \mathbf{y}) \cdot \mathbf{e}_{j}(t) d t=\sum_{l=1}^{n} \boldsymbol{\psi}_{j, l}\left(t, y_{l}\right) d W_{j, l}(t), \\
\boldsymbol{\Lambda}_{j}(t, \mathbf{p}) \cdot \boldsymbol{\xi}_{j}(t) d t=\sum_{l=1}^{n} \boldsymbol{\Lambda}_{j, l}\left(t, p_{l}\right) d Z_{j, l}(t),
\end{array}\right.
$$

where $\mathbf{e}_{j}$ and $\boldsymbol{\xi}_{j}$ are $n$-dimensional white noise processes; - stands for dot product. 


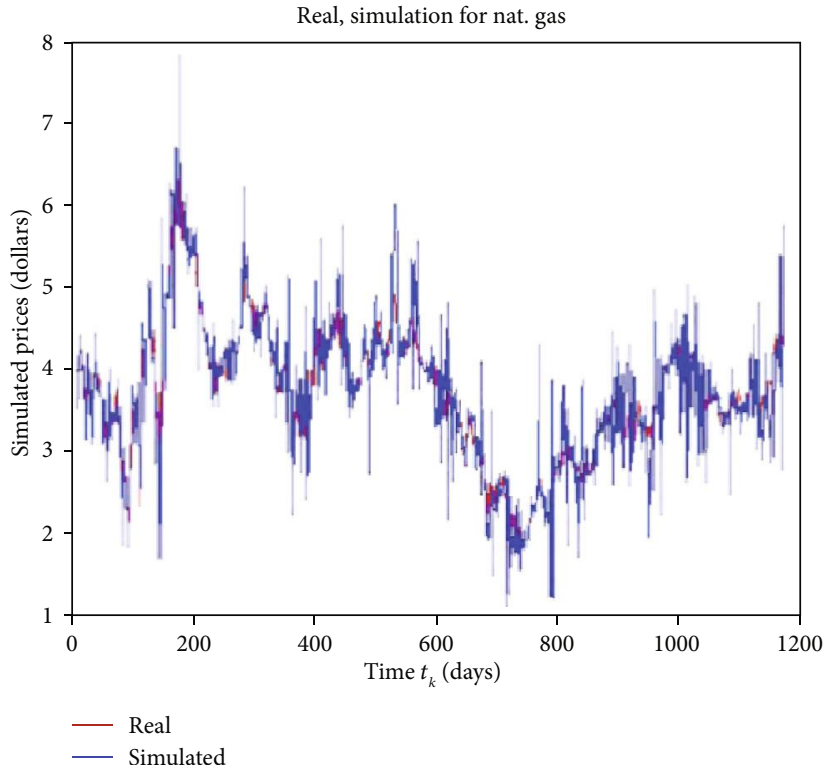

(a)

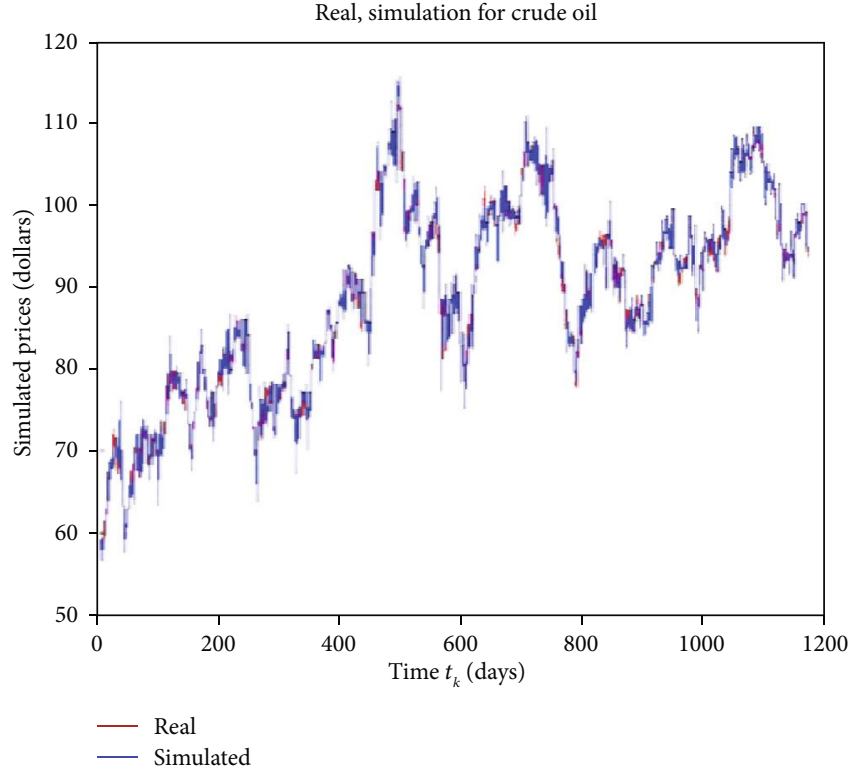

(b)

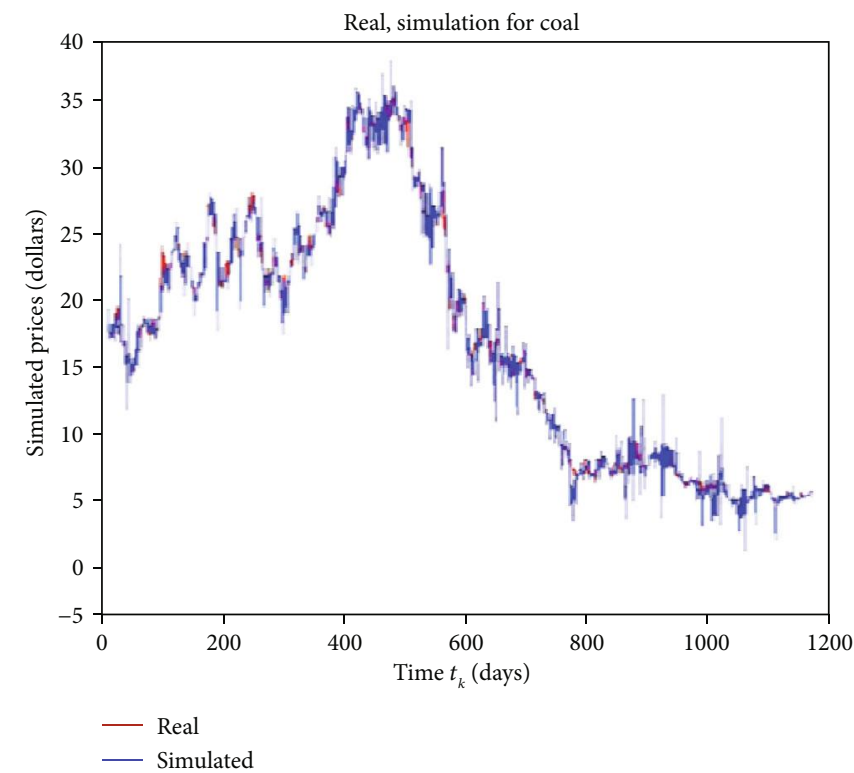

(c)

Figure 7: Real and simulated prices (without jump) for natural gas, crude oil, and coal.

TABLE 6: Some result for the jump times of the system $(\mathbf{y}, \mathbf{p})$.

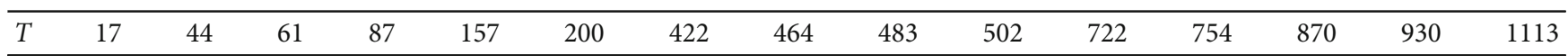

We assume that the interaction functions (10) and (11) have the following forms:

$$
\left\{\begin{array}{l}
\mathbf{g}(t, \mathbf{y})=\boldsymbol{\gamma}(t, \mathbf{y}) G(t, \mathbf{y}), \\
\mathbf{h}(t, \mathbf{p})=\boldsymbol{\lambda}(t, \mathbf{p}) \mathbf{H}(t, \mathbf{p}), \\
\boldsymbol{\psi}(t, y)=\boldsymbol{\gamma}(t, \mathbf{y}) \boldsymbol{\Psi}(t, \mathbf{y}), \\
\boldsymbol{\Lambda}(t, \mathbf{p})=\boldsymbol{\lambda}(t, \mathbf{p}) \boldsymbol{\Phi}(t, \mathbf{p}),
\end{array}\right.
$$

where $\mathbf{g}(t, \mathbf{y})=\left[\mathbf{g}_{1}(t, \mathbf{y}), \cdots, \mathbf{g}_{j}(t, \mathbf{y}), \cdots, \mathbf{g}_{n}(t, \mathbf{y})\right]^{\top}, \mathbf{h}(t, \mathbf{p})=$ $\left[\mathbf{h}_{1}(t, \mathbf{p}), \cdots, \mathbf{h}_{j}(t, \mathbf{p}), \cdots, \mathbf{h}_{n}(t, \mathbf{p})\right]^{\top}$ are defined in $(10), \boldsymbol{\psi}(t$, $\mathbf{y})=\left(\boldsymbol{\psi}_{j, l}(t, \mathbf{y})\right)_{n \times n}, \quad$ and $\boldsymbol{\Lambda}(t, \mathbf{p})=\left(\boldsymbol{\Lambda}_{j, l}(t, \mathbf{p})\right)_{n \times n}, \quad \boldsymbol{\gamma}(t, y)=$ $\operatorname{diag}\left(u_{1}-y_{1}, \cdots, u_{j}-y_{j}, \cdots, u_{n}-y_{n}\right)$ and $\lambda(t, p)=\operatorname{diag}\left(l_{1}+\right.$ $\left.p_{1}, \cdots, l_{j}+p_{j}, \cdots, l_{n}+p_{n}\right) ; \mathbf{G}$, and $\mathbf{H}$ are $n \times 1$ column vectors; $\boldsymbol{\Psi}=\operatorname{diag}\left(\boldsymbol{\psi}_{1}, \cdots, \psi_{j}, \cdots, \boldsymbol{\psi}_{n}\right)$ and $\boldsymbol{\Phi}=\operatorname{diag}\left(\boldsymbol{\Lambda}_{1}, \cdots, \boldsymbol{\Lambda}_{j}, \cdots\right.$, $\left.\Lambda_{n}\right)$ are block diagonal matrices; $\psi_{j}=\left[\psi_{j, 1}, \cdots, \psi_{j, l}, \cdots, \psi_{j, n}\right]$, $\boldsymbol{\Lambda}_{j}=\left[\boldsymbol{\Lambda}_{j, 1}, \cdots, \boldsymbol{\Lambda}_{j, l}, \cdots, \boldsymbol{\Lambda}_{j, n}\right]$. We also assume that $\mathbf{G}, \mathbf{H}, \boldsymbol{\Psi}$, and $\Phi$ satisfy the local Lipschitz condition. This assumption 
implies that $\mathbf{g}, \mathbf{h}, \boldsymbol{\psi}$, and $\boldsymbol{\Lambda}$ also satisfy local Lipschitz condition.
Thus, the interconnected energy commodity network system is described by

$$
\left\{\begin{array}{l}
d y_{j}=\left(u_{j}-y_{j}\right)\left[\left(\mu_{j}\left(v_{j}+y_{j}\right)+\mathbf{G}_{j}(t, \mathbf{y})\right) d t+\delta_{j, j} d W_{j, j}(t)+\sum_{l=1}^{n} \boldsymbol{\Psi}_{j, l}(t, \mathbf{y}) d W_{j, l}(t)\right], y_{j}\left(t_{0}\right)=y_{j 0}, \\
d p_{j}=\left(l_{j}+p_{j}\right)\left[\left(\alpha_{j}\left(y_{j}-p_{j}\right)+\mathbf{H}_{j}(t, \mathbf{p})\right) d t+\sigma_{j, j} d Z_{j, j}(t)+\sum_{l=1}^{n} \boldsymbol{\Phi}_{j, l}(t, \mathbf{p}) d Z_{j, l}(t)\right], p_{j}\left(t_{0}\right)=p_{j 0}, j \in I(1, n),
\end{array}\right.
$$

where the parameters $\mu_{j}>0 ; \alpha_{j}>0 ; u_{j}>0 ; v_{j} \geq 0 ; l_{j} \geq 0$; $\delta_{j, j}>0 ; \sigma_{j, j}>0$; and for $j \neq l, \delta_{j, l} \geq 0 ; \sigma_{j, l} \geq 0 ; j, l \in I(1, n)$; for $j \in I(1, n), \mathbf{W}_{j}$ and $\mathbf{Z}_{j}$ are $n$-dimensional independent Wiener processes defined on a filtered probability space $\left(\Omega, \mathscr{F},\left(\mathscr{F}_{t}\right)_{t \geq 0}, \mathscr{P}\right)$; for $l \neq i, \mathbb{E}\left[d W_{j, l} d W_{k, i}\right]=0$, and for $l=$ $i, \mathbb{E}\left[d W_{j, l} d W_{k, i}\right]=d t$; the filtration function $\left(\mathscr{F}_{t}\right)_{t \geq 0}$ is right continuous; for each $t \geq 0$, each $\mathscr{F}_{t}$ contains all $\mathscr{P}$-null sets in $\mathscr{F}$; the $n$-dimensional random vectors $\mathbf{y}\left(t_{0}\right)$ and $\mathbf{p}\left(t_{0}\right)$ are $\mathscr{F}_{t_{0}}$ measurable.
The network system of stochastic differential equations in (13) can be written as follows:

$$
\left\{\begin{array}{l}
d \mathbf{y}=\mathbf{a}(t, \boldsymbol{y}) d t+\mathbf{Y}(t, \mathbf{y}) d \mathbf{W}(t), \mathbf{y}\left(t_{0}\right)=\mathbf{y}_{0} \\
d \mathbf{p}=\mathbf{b}(t, \mathbf{y}, \mathbf{p}) d t+\boldsymbol{\sigma}(t, \mathbf{p}) d \mathbf{Z}(t), \mathbf{p}\left(t_{0}\right)=\mathbf{p}_{0}
\end{array}\right.
$$

where

$$
\left\{\begin{array}{c}
\mathbf{a}(t, \mathbf{y})=\left(\begin{array}{c}
\left(u_{1}-y_{1}\right)\left[\mu_{1}\left(v_{1}+y_{1}\right)+\mathbf{G}_{1}(t, \mathbf{y})\right] \\
\left(u_{2}-y_{2}\right)\left[\mu_{2}\left(v_{2}+y_{2}\right)+\mathbf{G}_{2}(t, \mathbf{y})\right] \\
\vdots \\
\left(u_{n}-y_{n}\right)\left[\mu_{n}\left(v_{n}+y_{n}\right)+\mathbf{G}_{n}(t, \mathbf{y})\right]
\end{array}\right), \mathbf{b}(t, \mathbf{y}, \mathbf{p})=\left(\begin{array}{c}
\left(l_{1}+p_{1}\right)\left[\alpha_{1}\left(y_{1}-p_{1}\right)+\mathbf{H}_{1}(t, \mathbf{p})\right] \\
\left(l_{2}+p_{2}\right)\left[\alpha_{2}\left(y_{2}-p_{2}\right)+\mathbf{H}_{2}(t, \mathbf{p})\right] \\
\vdots \\
\left(l_{n}+p_{n}\right)\left[\alpha_{n}\left(y_{n}-p_{n}\right)+\mathbf{H}_{n}(t, \mathbf{p})\right]
\end{array}\right), \\
\mathbf{Y}(t, y)=\operatorname{diag}\left(A_{1}(\mathbf{y}), \cdots, A_{j}(\mathbf{y}), . ., A_{n}(\mathbf{y})\right), \boldsymbol{\sigma}(t, \mathbf{p})=\operatorname{diag}\left(B_{1}(\mathbf{p}), \cdots, B_{j}(\mathbf{p}), \cdots, B_{n}(\mathbf{p})\right)
\end{array}\right.
$$

and

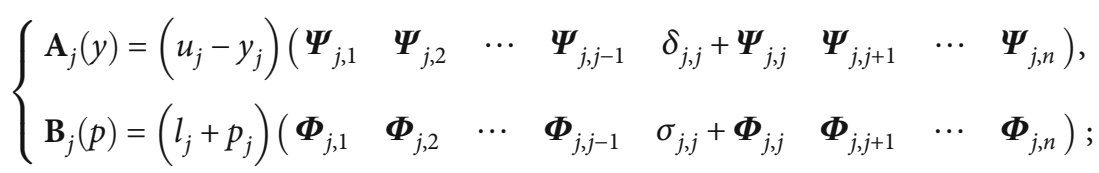

$\mathbf{W}=\left[\mathbf{W}_{1}, \cdots \mathbf{W}_{j}, \cdots, \mathbf{W}_{n}\right]^{T}$ and $\mathbf{Z}=\left[\mathbf{Z}_{1}, \cdots \mathbf{Z}_{j}, \cdots, \mathbf{Z}_{n}\right]^{T}$ are block matrices; $\mathbf{W}_{j}=\left[W_{j, 1}, \cdots W_{j, 2}, \cdots, W_{j, n}\right]^{T}, \quad \mathbf{Z}_{j}=\left[Z_{j, 1}\right.$, $\left.\cdots Z_{j, 2}, \cdots, Z_{j, n}\right]^{T}$; and $\mathbf{Y}(t, \mathbf{y}), \boldsymbol{\sigma}(t, \mathbf{p})$ are a $n \times n$ block matrix with each entries having order $1 \times n$.

In the next section, we outline the model validation problems of (14), namely, the existence and uniqueness of solution process.

\section{Mathematical Model Validation}

Here, we validate the mathematical model derived in Section 2. We note that the classical existence and uniqueness theo- rem [23-25] is not directly applicable to (14). We need to modify the existence and uniqueness results. The modification is based on Theorem 3.4 [23]. We show the global existence of the solution process of a system of differential equations (14). We note that the rate functions $\mathbf{a}, \mathbf{b}, \mathbf{Y}$, and $\boldsymbol{\sigma}$ in stochastic system of differential equations (14) do not satisfy the classical existence and uniqueness conditions [23]. However, these rate functions do satisfy the local Lipschitz condition. Therefore, we construct sequences of functions for the drift and diffusion coefficients of interconnected dynamic system (14) so that the classical conditions for existence and uniqueness theorem are applicable. The construction of modification scheme is as follows: First, we 
define a cylindrical subset $\left[t_{0}, \infty\right) \times U_{m}$ of $[0, \infty) \times \mathfrak{R}^{n}$ for $t_{0} \in[0, \infty), m \geq 1$, where $U_{m}$ is an $n$-dimensional sphere with radius $m$ defined by

$$
U_{m}=\mathbb{B}\left(\mathbf{x}_{0}, m\right)=\left\{\mathbf{x} \in \mathfrak{R}^{n}:\left\|\mathbf{x}-\mathbf{x}_{0}\right\|<m\right\},
$$

for any $m \geq 1$. We note that $U_{m}$ is inscribed in $n$-dimensional parallelepiped $\mathbb{R}\left(\mathbf{x}-\mathbf{x}_{0}, m\right)=[-m, m] \times \cdots \times[-m, m]$ in $\mathfrak{R}^{n}$.

The developed stochastic network model (14) can be written as:

$$
\left\{\begin{array}{l}
d \mathbf{y}=\mathbf{a}^{m}(t, \mathbf{y}) d t+\mathbf{Y}^{m}(t, \mathbf{y}) d \mathrm{~W}(t), \mathbf{y}\left(t_{0}\right)=\mathbf{y}_{0}, \\
d \mathbf{p}=\mathbf{b}^{m}(t, \boldsymbol{y}, \mathbf{p}) d t+\boldsymbol{\sigma}^{m}(t, \mathbf{p}) d \mathbf{Z}(t), \mathbf{p}\left(t_{0}\right)=\mathbf{p}_{0}
\end{array}\right.
$$

where

$$
\left\{\begin{array}{l}
\mathbf{a}^{m}(t, \mathbf{y})=\mathbf{a}(t, \mathbf{q}(\mathbf{y}, m)) \\
\mathbf{Y}^{m}(t, \mathbf{y})=\mathbf{Y}(t, \mathbf{q}(\mathbf{y}, m)) \\
\mathbf{b}^{m}(t, \mathbf{y}, \mathbf{p})=\mathbf{b}(t, \mathbf{q}(\mathbf{y}, m), \mathbf{q}(\mathbf{p}, m)) \\
\boldsymbol{\sigma}^{m}(t, \mathbf{p})=\boldsymbol{\sigma}(t, \mathbf{q}(\mathbf{p}, m))
\end{array}\right.
$$

Here, for each $j \in I(1, n)$ and $\mathbf{x} \in \mathfrak{R}^{n}$, we define $\mathbf{q}_{j}(\mathbf{x}, m)$ $=\max \left\{-m, \min \left\{x_{j}-x_{0 j}, m\right\}\right\}$. Hence, $\mathbf{q}(\mathbf{x}, m)=$ $\left[\mathbf{q}_{1}(\mathbf{x}, m), \cdots, \mathbf{q}_{j}(\mathbf{x}, m), \cdots, \mathbf{q}_{n}(\mathbf{x}, m)\right]^{T}$.

Remark 1. We observe that $\mathbf{q}(\mathbf{x}, m)$ satisfies the global Lipschitz condition on $\mathfrak{R}^{n}$ with a Lipschitz constant 1 . Using this, together with the local Lipschitz condition assumption on the drift and diffusion coefficients of stochastic differential equations (14), the modified rate coefficient functions in (18) satisfy the classical existence and uniqueness conditions $[26,27]$. Thus, its solution is denoted by $\left(\mathbf{y}_{m}, \mathbf{p}_{m}\right)$, for $m \geq 1$. Moreover, $(\mathbf{y}, \mathbf{p})$ is nonnegative whenever $\mathbf{y}_{0}, \mathbf{p}_{0} \in \mathfrak{R}_{+}^{n}$.

Now, we apply Theorems 3.4 and 3.5 of [26] in the context of the modified system of stochastic differential equations (18) and Remark 1 to establish the global existence of solution of stochastic differential equations in (13). For this purpose, we outline the argument used in the proof of these theorems. In addition to the local Lipschitz conditions on the drift and diffusion coefficients, we further impose the following hypothesis on the coefficients:

$\left(\mathbf{H}_{1}\right)$

$$
\left\{\begin{array}{l}
\left|\mathbf{g}_{j}(t, \mathbf{y})\right| \leq a_{1, j}+\kappa_{j}\|\mathbf{y}\|, \\
\left|\boldsymbol{h}_{j}(t, \mathbf{p})\right| \leq a_{1, j}^{\prime}+\gamma_{j}\|\mathbf{p}\|, \\
\left|\boldsymbol{\psi}_{j, l}(t, \mathbf{y})\right| \leq a_{2, j}+\tilde{\delta}_{j, l}\|\mathbf{y}\|, \\
\left|\boldsymbol{\Lambda}_{j, l}(t, \mathbf{p})\right| \leq a_{2, j}^{\prime}+\tilde{\sigma}_{j, l}\|\mathbf{p}\|,
\end{array}\right.
$$

where for $i \in I(1,2), a_{i, j}, a_{i, j}$ are nonnegative; $\kappa_{j}, \gamma_{j}, \tilde{\delta}_{j, l}$, $\tilde{\sigma}_{j, l} \in \mathfrak{R}_{+}$. From (18), we further remark that the dynamic of the mean spot price vector $\mathbf{y}$ is decoupled with the dynamic of spot price $p$. Now, we first apply Theorems 3.4 and 3.5 of [23] in the context of modified system of stochastic differential equations $(18)$ and hypothesis $\left(\mathbf{H}_{1}\right)$ to establish the global existence of solution of the completely decoupled subsystem of stochastic differential equations in (18). For this purpose, we outline the argument used in the proof of these theorems.

Definition 2. Let $\tau_{m}$ be the first exit time of the solution process $\mathbf{y}_{m}$ from the set $\mathbb{B}\left(\mathbf{y}_{0}, m\right)$. Define $\boldsymbol{\tau}$ to be the (finite or infinite) limit of the monotone increasing sequence $\boldsymbol{\tau}_{m}$ as $m \longrightarrow \infty$.

$$
\boldsymbol{\tau}=\lim _{m \rightarrow \infty} \boldsymbol{\tau}_{m}
$$

We wish to show that

$$
\operatorname{Prob}(\boldsymbol{\tau}=\infty)=1
$$

In the following, we present a result that is parallel to Theorem 3.5 [26] in the context of the completely decoupled subsystem of stochastic differential equation (14). For this purpose, it is enough to exhibit the global existence result for the transformed system (18).

Lemma 3. For $m \geq 1$, and $y_{0} \in \mathfrak{R}_{+}^{n}$, let $\boldsymbol{y}_{m}(t)=y_{m}\left(t, t_{0}\right.$, $\left.y_{0}\right)$ be the solution of the completely decoupled subsystem of (18), and let the hypothesis $\left(\boldsymbol{H}_{1}\right)$ be satisfied. Let $V_{1}$ be a function defined on $\left[t_{0}, \infty\right) \times \mathfrak{R}_{+}^{n}$ into $\mathfrak{R}_{+}$defined by

$$
V_{1}(t, y)=\ln \left(\|\mathbf{y}\|^{2}+e\right)
$$

Then, there exists some constant $\mathbf{c}_{1}>0$ such that

$$
\left\{\begin{array}{l}
\mathbf{L} V_{1} \leq \mathbf{c}_{1} V_{1}, \\
V_{1, m}=\inf _{\|y\|>m} V_{1}(t, \mathbf{y}) \longrightarrow \text { oas } m \longrightarrow \infty,
\end{array}\right.
$$

where $\mathbf{L}$ is the differential operator with respect to (14); $e=\exp (1)$.

Moreover, the global existence of solution of the completely decoupled subsystem of (14) is follows.

Proof. It is obvious that $V_{1} \in \mathscr{C}_{1,2}$ on $\left[t_{0}, \infty\right) \times \mathfrak{R}_{+}^{n} \rightarrow \mathfrak{R}_{+}$. In fact, $\left(\partial V_{1}(t, \mathbf{y})\right) / \partial y_{j}=2 y_{j} /(\mathbf{y}+e), \quad \partial^{2} V_{1}(t, \mathbf{y}) / \partial y_{j}^{2}=(2 /$ $(\mathbf{y}+e))-\left(4 y_{j}^{2} /\left(\|\mathbf{y}\|^{2}+e\right)^{2}\right), \quad$ and $\partial^{2} V_{1}(t, \mathbf{y}) / \partial y_{i} y_{j}=-4 y_{i} y_{j} /$ $\left(\|\mathbf{y}\|^{2}+e\right)^{2}$ exist and are continuous functions defined on 
$\left[t_{0}, \infty\right) \times \mathfrak{R}_{+}^{n} \longrightarrow \mathfrak{R}$. Moreover, the $\mathbf{L}$ operator with respect to the completely decoupled component is as follows:

$$
\begin{aligned}
& \mathbf{L} V_{1}(t, \mathbf{y})=\sum_{j=1}^{n}\left[\mu_{j}\left(u_{j}-y_{j}\right)\left(v_{j}+y_{j}\right)+\mathbf{g}_{j}(t, \mathbf{y})\right] \frac{\partial V_{1}(t, y)}{\partial y_{j}} \\
& +\frac{1}{2} \sum_{j=1}^{n}\left[\left[\delta_{j, j}\left(u_{j}-y_{j}\right)+\boldsymbol{\psi}_{j j}(t, \mathbf{y})\right]^{2}\right. \\
& \left.+\sum_{l \neq j}^{n} \psi_{j, l}^{2}(t, \mathbf{y})\right] \frac{\partial^{2} V_{1}(t, y)}{\partial y_{j}^{2}}+\frac{1}{2} \sum_{i=1}^{n} \sum_{j=1}^{n} \\
& \cdot\left[\sum_{l \neq \mathrm{i}, j}^{n} \boldsymbol{\psi}_{i, l}(t, \mathbf{y}) \boldsymbol{\psi}_{j, l}(t, \mathbf{y})+2\left[\delta_{i, i}\left(u_{i}-y_{i}\right)+\boldsymbol{\psi}_{i, i}\right] \boldsymbol{\psi}_{j, i}\right] \\
& \frac{\partial^{2} V_{1}(t, y)}{\partial y_{i} y_{j}} \\
& =\sum_{j=1}^{n} \mu_{j}\left(-\left[y_{j}-\left(\frac{u_{j}-v_{j}}{2}\right)\right]^{2}+\left(\frac{u_{j}+v_{j}}{2}\right)^{2}\right) \frac{2 y_{j}}{\left(\|\mathbf{y}\|^{2}+e\right)} \\
& +\sum_{j=1}^{n} \frac{2 \mathbf{g}_{j}(t, \mathbf{y}) y_{j}}{\left(\|\mathbf{y}\|^{2}+e\right)}+\frac{1}{2} \sum_{j=1}^{n}\left[\delta_{j, j}\left(u_{j}-y_{j}\right)+\boldsymbol{\psi}_{j, j}\right]^{2} \\
& \cdot\left(\frac{2}{\left(\|\mathbf{y}\|^{2}+e\right)}-\frac{4 y_{j}^{2}}{\left(\|\mathbf{y}\|^{2}+e\right)^{2}}\right)+\frac{1}{2} \sum_{j=1}^{n} \sum_{l \neq j}^{n} \psi_{j, l}^{2}(t, \mathbf{y}) \\
& \cdot\left(\frac{2}{\left(\|\mathbf{y}\|^{2}+e\right)}-\frac{4 y_{j}^{2}}{\left(\|\mathbf{y}\|^{2}+e\right)^{2}}\right)-\frac{1}{2} \sum_{i=1}^{n} \sum_{j=1}^{n} \\
& j \neq i \\
& {\left[\sum_{l \neq i, j}^{n} \boldsymbol{\psi}_{i, l}\left(t, y_{l}\right) \boldsymbol{\psi}_{j, l}(t, \mathbf{y})+2\left[\delta_{i, i}\left(u_{i}-y_{i}\right)+\boldsymbol{\psi}_{i, i}\right] \boldsymbol{\psi}_{j, i}\right]} \\
& \cdot \frac{4 y_{i} y_{j}}{\left(\|\mathbf{y}\|^{2}+e\right)^{2}} \leq 2 \sum_{j=1}^{n} \mu_{j}\left(\frac{u_{j}+v_{j}}{2}\right)^{2} \frac{y_{j}}{\left(\|\mathbf{y}\|^{2}+e\right)} \\
& +\sum_{j=1}^{n} \frac{2 \mathbf{g}_{j}(t, \mathbf{y}) y_{j}}{\left(\|\mathbf{y}\|^{2}+e\right)}+\sum_{j=1}^{n} \frac{\left[\delta_{j, j}\left(u_{j}-y_{j}\right)+\psi_{j j}(t, \mathbf{y})\right]^{2}}{\left(\|\mathbf{y}\|^{2}+e\right)} \\
& +\sum_{j=1}^{n} \sum_{l \neq j}^{n} \frac{\psi_{j, l}^{2}(t, y)}{\left(\|\mathbf{y}\|^{2}+e\right)}-\frac{1}{2} \sum_{i=1}^{n} \sum_{j=1}^{n} \\
& j \neq i \\
& \cdot\left[\sum_{l \neq i, j}^{n} \boldsymbol{\psi}_{i, l}\left(t, y_{l}\right) \boldsymbol{\psi}_{j, l}(t, \mathbf{y})+2\left[\delta_{i, i}\left(u_{i}-y_{i}\right)+\boldsymbol{\psi}_{i, i}\right] \boldsymbol{\psi}_{j, i}\right] \\
& \cdot \frac{4 y_{i} y_{j}}{\left(\|\mathbf{y}\|^{2}+e\right)^{2}} \leq 2 \sum_{j=1}^{n} \mu_{j}\left(\frac{u_{j}+v_{j}}{2}\right)^{2} \\
& +\sum_{j=1}^{n} \frac{\left(a_{1, j}+\kappa_{j}\|\mathbf{y}\|^{2}\right)^{2}+y_{j}^{2}}{\left(\|\mathbf{y}\|^{2}+e\right)} \\
& +2 \sum_{j=1}^{n}\left[\delta_{j, j}^{2}\left(u_{j}+1\right)^{2}+\left(a_{2, j}+\tilde{\delta}_{j, j}\right)^{2}\right] \\
& +\sum_{j=1}^{n} \sum_{l \neq j}^{n}\left(a_{2, j}+\tilde{\delta}_{j, l}\right)^{2}+2 \sum_{i=1}^{n} \sum_{j \neq i}^{n} \sum_{l \neq i, j}^{n}\left(a_{2, i}+\tilde{\delta}_{i, l}\right)\left(a_{2, j}+\tilde{\delta}_{j, l}\right) \\
& +4 \sum_{i=1}^{n} \sum_{j \neq i}^{n}\left(a_{2, j}+\tilde{\delta}_{j, i}\right)\left[\delta_{i, i}\left(u_{i}+1\right)+\left(a_{2, i}+\tilde{\delta}_{i, i}\right)\right] \\
& \leq c_{1} V_{1}(t, \mathbf{y}) \text {, }
\end{aligned}
$$

where

$$
\begin{aligned}
c_{1}= & 2 \sum_{j=1}^{n} \mu_{j}\left(\frac{u_{j}+v_{j}}{2}\right)^{2}+1+\sum_{j=1}^{n}\left(a_{1, j}+\kappa_{j}\right)^{2} \\
& +2 \sum_{j=1}^{n}\left[\delta_{j, j}^{2}\left(u_{j}+1\right)^{2}+\left(a_{2, j}+\tilde{\delta}_{j, j}\right)^{2}\right] \\
& +\sum_{j=1}^{n} \sum_{l \neq j}^{n}\left(a_{2, j}+\tilde{\delta}_{j, l}\right)^{2}+2 \sum_{i=1}^{n} \sum_{j \neq i}^{n} \sum_{l \neq i, j}^{n}\left(a_{2, i}+\tilde{\delta}_{i, l}\right)\left(a_{2, j}+\tilde{\delta}_{j, l}\right) \\
& +4 \sum_{i=1}^{n} \sum_{j \neq i}^{n}\left(a_{2, j}+\tilde{\delta}_{j, i}\right)\left[\delta_{i, i}\left(u_{i}+1\right)+\left(a_{2, i}+\tilde{\delta}_{i, i}\right)\right] .
\end{aligned}
$$

Furthermore, $\inf _{\|\mathbf{y}\|>m} V_{1}(t, \mathbf{y}) \longrightarrow \infty$ as $m \longrightarrow \infty$.

To show that $\operatorname{Prob}(\tau=\infty)=1$, we define a function

$$
V(t, \mathbf{y})=V_{1}(t, \mathbf{y}) \exp \left\{-\mathbf{c}_{1}\left(t-t_{0}\right)\right\}
$$

It is obvious that $\mathbf{L} V \leq 0$. By defining $\tau_{m}(t)=\min \left(\tau_{m}, t\right)$; $\mathscr{Y}(t)=\mathbf{y}_{m}(t)$ for $t<\tau_{m}$; and imitating the argument of Lemma 4 [23], we have

$$
\mathbb{E}\left\{V_{1}\left(\tau_{m}(t), \mathcal{Y}\left(\tau_{m}(t)\right)\right\} \leq e^{c_{1}\left(t-t_{0}\right)} \mathbb{E} V_{1}\left(t_{0}, \mathbf{y}\left(t_{0}\right)\right)\right.
$$

Hence,

$$
\begin{aligned}
\operatorname{Prob}\left\{\tau_{m} \leq t\right\} & \leq \frac{e^{\mathfrak{c}_{1}\left(t-t_{0}\right)} \mathbb{E} V_{1}\left(t_{0}, \mathcal{Y}\left(t_{0}\right)\right)}{\inf _{\|y\|>m, u>t_{0}} V_{1}(u, y)} \longrightarrow 0 \text { as } m \\
& \longrightarrow \infty \text { by }(24) .
\end{aligned}
$$

The global existence and uniqueness of the solution of the first component of (18) follows by letting $m \longrightarrow \infty$. Hence, from this and the fact that the solution process of transformed system (18) is almost surely identical with the solution process of the original system (14), we conclude the global existence and uniqueness of (14).

Following the idea of Lemma 3, we present a global existence and uniqueness of solution of the system of stochastic differential equations governing the subsystem $p$ in (14).

Lemma 4. For $m \geq 1$, and $\boldsymbol{y}_{0}, \mathbf{p}_{0} \in \mathfrak{R}_{+}^{n}$, let $\mathbf{p}_{m}(t)=\mathbf{p}_{m}\left(t, t_{0}, \mathbf{p}_{0}\right)$ be the solution of the system of stochastic differential equations governing the subsystem $\mathbf{p}$ described in (18), and let the hypothesis $\left(\boldsymbol{H}_{1}\right)$ be satisfied. Let $V_{2}$ be a nonnegative function on $\left[t_{0}, \infty\right) \times \mathfrak{R}_{+}^{n}$ into $\mathfrak{R}_{+}$defined by

$$
V_{2}(t, \mathbf{p})=\ln \left(\|\mathbf{p}\|^{2}+e\right)+\sum_{j=1}^{n} \frac{\alpha_{j}}{2} \int_{t}^{T}\left(y_{j}(s)+l_{j}\right)^{2} d s,
$$


Then, there exist a constant $c>0$ such that

$$
\left\{\begin{array}{l}
\mathbf{L} V_{2} \leq c V_{2} \\
V_{2, m}=\inf _{\|\boldsymbol{p}\|>m} V_{2}(t, \boldsymbol{p}) \longrightarrow \infty \text { as } m \longrightarrow \infty
\end{array}\right.
$$

where $\boldsymbol{L}$ is the differential operator with respect to (14); $e=\exp (1)$.

Moreover, the global existence of the solution of the system of stochastic differential equations governing the subsystem $p$ described in (14) is as follows.

Proof. It is obvious that $V_{2} \in \mathscr{C}_{1,2}$ on $\left[t_{0}, \infty\right) \times \mathfrak{R}_{+}^{n} \longrightarrow \mathfrak{R}_{+}$. In fact, $\partial V_{2}(t, \mathbf{p}) / \partial t=-\sum_{j=1}^{n}\left(\alpha_{j} / 2\right)\left(y_{j}(t)+l_{j}\right)^{2}, \partial V_{2}(t, \mathbf{p}) / \partial p_{j}=$ $2 p_{j} /\left(\|\mathbf{p}\|^{2}+e\right), \quad \partial^{2} V_{2}(t, \mathbf{p}) / \partial p_{j}^{2}=\left(2 /\left(\|\mathbf{p}\|^{2}+e\right)\right)-\left(4 p_{j}^{2} /\right.$ $\left.\left(\|\mathbf{p}\|^{2}+e\right)^{2}\right)$, and $\partial^{2} V_{2}(t, \mathbf{p}) / \partial p_{i} p_{j}=-4 p_{i} p_{j} /\left(\|\mathbf{p}\|^{2}+e\right)^{2}$ exist and are continuous functions defined on $\left[t_{0}, \infty\right) \times \mathfrak{R}_{+}^{n} \longrightarrow$ $\mathfrak{R}$. Moreover, the $\mathbf{L}$ operator with respect to the system of stochastic differential equations governing the subsystem $\mathbf{p}$ described in (14) is obtained as follows:

$$
\begin{aligned}
\mathbf{L V}_{2}(t, \mathbf{p})= & -\sum_{j=1}^{n} \frac{\alpha_{j}}{2}\left(y_{j}(t)+l_{j}\right)^{2}+\sum_{j=1}^{n}\left[\alpha_{j}\left(y_{j}-p_{j}\right)\left(l_{j}+p_{j}\right)\right. \\
& \left.+\mathbf{h}_{j}(t, \mathbf{p})\right] \frac{2 p_{j}}{\left(\|\mathbf{p}\|^{2}+e\right)}, \\
& +\frac{1}{2} \sum_{j=1}^{n}\left[\left[\sigma_{j, j}\left(l_{j}+p_{j}\right)+\boldsymbol{\Lambda}_{j, j}(t, \mathbf{p})\right]^{2}\right. \\
& \left.+\sum_{l \neq j}^{n} \boldsymbol{\Lambda}_{j, l}(t, \mathbf{p})^{2}\right]\left[\frac{2}{\left(\|\mathbf{p}\|^{2}+e\right)}-\frac{4 p_{j}^{2}}{\left(\|\mathbf{p}\|^{2}+e\right)^{2}}\right] \\
& +\frac{1}{2} \sum_{i=1}^{n} \sum_{j=1}^{n}\left[\sum_{l=1}^{n} \boldsymbol{\Lambda}_{i, l} \boldsymbol{\Lambda}_{j, l}+2\left[\sigma_{i, i}\left(l_{i}+p_{i}\right)+\boldsymbol{\Lambda}_{i, i} \boldsymbol{\Lambda}_{j, i}\right]\right. \\
& +\left[-\frac{4 p_{i} p_{j}}{\left(\|\mathbf{p}\|^{2}+e\right)^{2}}\right] \\
= & -\sum_{j=1}^{n} \frac{\alpha_{j}}{2}\left(y_{j}+l_{j}\right)^{2}+\sum_{j=1}^{n} \alpha_{j}\left(-\left[p_{j}-\frac{y_{j}-l_{j}}{2}\right]^{2}\right. \\
& \left.+\left(\frac{y_{j}+l_{j}}{2}\right)^{2}\right) \frac{2 p_{j}}{\left(\|\mathbf{p}\|^{2}+e\right)} \\
& +\sum_{j=1}^{n} \frac{2 \mathbf{h}_{j}(t, \mathbf{p}) p_{j}}{\left(\|\mathbf{p}\|^{2}+e\right)}+\frac{1}{2} \sum_{j=1}^{n}\left[\sigma_{j, j}\left(l_{j}+p_{j}\right)+\boldsymbol{\Lambda}_{j, j}(t, \mathbf{p})\right]^{2} \\
& +\left(\frac{2}{\left(\|\mathbf{p}\|^{2}+e\right)}-\frac{4 p_{j}^{2}}{\left(\|\mathbf{p}\|^{2}+e\right)^{2}}\right) \\
& +\frac{1}{2} \sum_{j=1}^{n} \sum_{l \neq j}^{n} \boldsymbol{\Lambda}_{j, l}(t, \mathbf{p})^{2}\left(\frac{2}{\left(\|\mathbf{p}\|^{2}+e\right)}-\frac{4 p_{j}^{2}}{\left(\|\mathbf{p}\|^{2}+e\right)^{2}}\right)
\end{aligned}
$$

$$
\begin{aligned}
& -\frac{1}{2} \sum_{i=1}^{n} \sum_{\substack{j=1 \\
j \neq i}}^{n}\left[\sum_{l=1}^{n} \boldsymbol{\Lambda}_{i, l} \boldsymbol{\Lambda}_{j, l}+2\left[\sigma_{i, i}\left(l_{i}+p_{i}\right)+\boldsymbol{\Lambda}_{i, i} \boldsymbol{\Lambda}_{j, i}\right]\right. \\
& \cdot \frac{4 p_{i} p_{j}}{\left(\|\mathbf{p}\|^{2}+e\right)^{2}} \leq-\sum_{j=1}^{n} \frac{\alpha_{j}}{2}\left(y_{j}+l_{j}\right)^{2} \\
& +\sum_{j=1}^{n} \frac{\alpha_{j}}{2}\left(y_{j}+l_{j}\right)^{2} \frac{p_{j}}{\left(\|\mathbf{p}\|^{2}+e\right)} \\
& +\sum_{j=1}^{n} \frac{\left[a_{1, j}^{\prime}+\gamma_{j}\|\mathbf{p}\|\right]^{2}+p_{j}^{2}}{\left(\|\mathbf{p}\|^{2}+e\right)} \\
& +\sum_{j=1}^{n} \frac{\left[\sigma_{j, j}\left(l_{j}+p_{j}\right)+\boldsymbol{\Lambda}_{j, j}(t, \mathbf{p})\right]^{2}}{\left(\|\mathbf{p}\|^{2}+e\right)} \\
& +\sum_{j=1}^{n} \sum_{l \neq j}^{n}\left(a_{2, j}^{\prime}+\tilde{\sigma}_{j, l}\right)^{2}+2 \sum_{i=1}^{n} \sum_{j \neq i}^{n} \sum_{l \neq i, j}^{n}\left(a_{2, i}^{\prime}+\tilde{\sigma}_{i, l}\right) \\
& \cdot\left(a_{2, j}^{\prime}+\tilde{\sigma}_{j, l}\right)+4 \sum_{i=1}^{n} \sum_{j \neq i}^{n}\left(a_{2, j}^{\prime}+\tilde{\sigma}_{j, i}\right) \\
& \cdot\left[\sigma_{i, i}\left(l_{i}+1\right)+\left(a_{2, i}^{\prime}+\tilde{\sigma}_{i, i}\right)\right] \leq c V_{2}(t, \mathbf{p}),
\end{aligned}
$$

where

$$
\begin{aligned}
c= & +\sum_{j=1}^{n}\left[a_{1, j}^{\prime}+\gamma_{j}\right]^{2}+2 \sum_{j=1}^{n}\left[\sigma_{j, j}^{2}\left(l_{j}+1\right)^{2}+\left(a_{2, j}^{\prime}+\tilde{\sigma}_{j, j}\right)^{2}\right] \\
& +\sum_{j=1}^{n} \sum_{l \neq j}^{n}\left(a_{2, j}^{\prime}+\tilde{\sigma}_{j, l}\right)^{2}+2 \sum_{i=1}^{n} \sum_{j \neq i}^{n} \sum_{l \neq i, j}^{n}\left(a_{2, i}^{\prime}+\tilde{\sigma}_{i, l}\right)\left(a_{2, j}^{\prime}+\tilde{\sigma}_{j, l}\right) \\
& +4 \sum_{i=1}^{n} \sum_{j \neq i}^{n}\left(a_{2, j}^{\prime}+\tilde{\sigma}_{j, i}\right)\left[\sigma_{i, i}\left(l_{i}+1\right)+\left(a_{2, i}^{\prime}+\tilde{\sigma}_{i, i}\right)\right] .
\end{aligned}
$$

Furthermore, $\inf _{\|p\|>m} V_{2}(t, \mathbf{p}) \longrightarrow \infty$ as $m \longrightarrow \infty$. Following the final argument used in proving the global existence of $y$ in Lemma 3, we conclude that there exists a unique global solution to the interconnected system of stochastic differential (14).

In the next section, we discuss the case where we incorporate jump process into the system $(\mathbf{y}, \mathbf{p})$.

\section{Energy Commodity Model with and without Jumps}

Due to random interventions that affects the price of energy commodities, we introduce random perturbations described by a continuous jump in our model. We follow the approach discussed in $[28,29]$ where a class of stochastic hybrid dynamic processes is investigated. 
Let $K \geq 0$ be the number of jumps on the interval $\left[t_{0}, T\right]$, for $T>0$. For $K \neq 0$, let $T_{1}, \cdots, T_{K}$ be the jump times over a time interval $\left[t_{0}, T\right]$ such that $t_{0}=T_{0} \leq T_{1}<\cdots<T_{K} \leq T$, where $T_{i}$ denotes the time at which the ith jump occurred in the system $(\mathbf{y}, \mathbf{p})$. For $K=0$, no jump has occurred on the interval $\left[t_{0}, T\right]$. We denote the $i$ th subinterval by $T_{i-1}$ $\leq t<T_{i}$. Knowing the global existence and uniqueness solution process of the system (14) on the interval $\left[t_{0}, T\right]$,
$T>0$ in Section 3, for $i \in I\left(1, K^{*}\right)$, where $K^{*}=K$ if $T_{K}=T$, and $K^{*}=K+1$ if $T_{K}<T$ and $K \neq 0$, we consider the solution process on each subinterval $\left[T_{i-1}, T_{i}\right)$, between jumps. For $i \in I\left(1, K^{*}\right)$ and $K=0$, we have $I(1, K)=\varnothing$ and $I\left(1, K^{*}\right)=\{1\}$. In this case, we consider the solution process on $\left[t_{0}, T\right]$. The interconnected system is governed by the following system of continuous time stochastic process:

$$
\left\{\begin{array}{l}
d \mathbf{y}^{i-1}=\mathbf{a}^{i-1}(t, \mathbf{y}) d t+\mathbf{Y}^{i-1}(t, \mathbf{y}) d \mathbf{W}(t), \mathbf{y}\left(T_{i-1}\right)=\mathbf{y}^{i-1}, t \in\left[T_{i-1}, T_{i}\right) \\
d \mathbf{p}^{i-1}=\mathbf{b}^{i-1}(t, \mathbf{y}, \mathbf{p}) d t+\boldsymbol{\sigma}^{i-1}(t, \mathbf{p}) d \mathbf{Z}(t), \mathbf{p}\left(T_{i-1}\right)=\mathbf{p}^{i-1}, t \in\left[T_{i-1}, T_{i}\right), i \in I\left(1, K^{*}\right) \\
\mathbf{y}^{i}=\Pi^{i} \mathbf{y}^{i-1}\left(T_{i}^{-}, T_{i-1}, \mathbf{y}^{i-1}\right), \\
\mathbf{p}^{i}=\boldsymbol{\Theta}^{i} \mathbf{p}^{i-1}\left(T_{i}^{-}, T_{i-1}, \mathbf{y}^{i-1}, \mathbf{p}^{i-1}\right),
\end{array}\right.
$$

where

$$
\begin{aligned}
& \Pi^{i}=\operatorname{diag}\left(\pi_{1}^{i}, \pi_{2}^{i}, \cdots, \pi_{n}^{i}\right), \\
& \Theta^{i}=\operatorname{diag}\left(\theta_{1}^{i}, \theta_{2}^{i}, \cdots, \theta_{n}^{i}\right),
\end{aligned}
$$

$\left(\mathbf{y}^{i-1}\left(t, T_{i-1}, \mathbf{y}^{i-1}\right), \mathbf{p}^{i-1}\left(t, T_{i-1}, \mathbf{y}^{i-1}, \mathbf{p}^{i-1}\right)\right)$ is the solution of the system of equation (34); for $K \neq 0$ and $i \in I\left(1, K^{*}\right), \Pi^{i}$ and $\Theta^{i}$ are jump coefficient matrices. These jump coefficients are estimated by $\widehat{\pi}_{j}^{i}=y_{j}\left(T_{i}\right) / \lim _{t \rightarrow T_{i}^{-}} y_{j}^{i-1}\left(t, T_{i-1}, \mathbf{y}^{i-1}\right) ; \widehat{\theta}_{j}^{i}=p_{j}$ $\left(T_{i}\right) / \lim _{t \rightarrow T_{i}^{-}} p_{j}^{i-1}\left(t, T_{i-1}, \mathbf{y}^{i-1}, \mathbf{p}^{i-1}\right) \quad$ for $\quad j \in I(1, n)$. Following the approach in $[28,29]$, the solution of (34) takes the following representation:

$$
\begin{aligned}
& \mathbf{y}\left(t, t_{0}, \mathbf{y}_{0}\right)=\left\{\begin{array}{l}
\mathbf{y}^{0}\left(t, t_{0}, \mathbf{y}_{0}\right), \mathbf{y}\left(t_{0}\right)=\mathbf{y}_{0}, t_{0} \leq t<T_{1}, \\
\mathbf{y}^{1}\left(t, T_{1}, \mathbf{y}^{1}\right), \mathbf{y}^{1}=\mathbf{y}\left(T_{1}\right), T_{1} \leq t<T_{2}, \\
\cdots \\
\mathbf{y}^{i-1}\left(t, T_{i-1}, \mathbf{y}^{i-1}\right), \mathbf{y}^{i-1}=\mathbf{y}\left(T_{i-1}\right), T_{i-1} \leq t<T_{i}, \\
\cdots \\
\mathbf{y}^{K}\left(t, T_{K}, \mathbf{y}^{K}\right), \mathbf{y}^{K}=\mathbf{y}\left(T_{K}\right), T_{K} \leq t \leq T,
\end{array}\right. \\
& p\left(t, t_{0}, y_{0}, p_{0}\right)=\left\{\begin{array}{l}
\mathbf{p}^{0}\left(t, t_{0}, \mathbf{y}_{0}, \mathbf{p}_{0}\right), \mathbf{p}\left(t_{0}\right)=\mathbf{p}_{0}, t_{0} \leq t<T_{1}, \\
\mathbf{p}^{1}\left(t, T_{1}, \mathbf{y}^{1}, \mathbf{p}^{1}\right), \mathbf{p}^{1}=\mathbf{p}\left(T_{1}\right), T_{1} \leq t<T_{2}, \\
\cdots \\
\mathbf{p}^{i-1}\left(t, T_{i-1}, \mathbf{y}^{i-1}, \mathbf{p}^{i-1}\right), \mathbf{p}^{i-1}=\mathbf{p}\left(T_{i-1}\right), T_{i-1} \leq t<T_{i}, \\
\cdots \\
\mathbf{p}^{K}\left(t, T_{K}, \mathbf{y}^{K}, \mathbf{p}^{K}\right), \mathbf{p}^{K}=\mathbf{p}\left(T_{K}\right), T_{K} \leq t \leq T,
\end{array}\right.
\end{aligned}
$$

Remark 5. In case of no jump, that is $K=0$, (34) and (36) reduce to

$$
\left\{\begin{array}{l}
d \mathbf{y}=\mathbf{a}(t, \mathbf{y}) d t+\mathbf{Y}(t, \mathbf{y}) d \mathbf{W}(t), \mathbf{y}\left(t_{0}\right)=\mathbf{y}_{0}, \\
d \mathbf{p}=\mathbf{b}(t, \mathbf{y}, \mathbf{p}) d t+\boldsymbol{\sigma}(t, \mathbf{p}) d \mathbf{Z}(t), \mathbf{p}\left(t_{0}\right)=\mathbf{p}_{0}, t_{0} \leq t \leq T,
\end{array}\right.
$$

$$
\left\{\begin{array}{l}
\mathbf{y}\left(t, t_{0}, \mathbf{y}_{0}\right), \quad \mathbf{y}\left(t_{0}\right)=\mathbf{y}_{0}, \\
\mathbf{p}\left(t, t_{0}, \mathbf{y}_{0}, \mathbf{p}_{0}\right), \quad \mathbf{p}\left(t_{0}\right)=\mathbf{p}_{0}, t_{0} \leq t<T,
\end{array}\right.
$$

respectively.

\section{Discrete-Time Dynamic Model for Local Sample Mean and Covariance Processes}

In this section, we extend the discrete-time dynamic model for local sample mean and variance processes given in Otunuga et al. $[16,17]$ to a multivariate case including the jump process. The development of idea and model of statistic for mean and covariance processes was motivated by the state and parameter estimation problems of the continuous time nonlinear stochastic dynamic model of the energy commodity market network. For this purpose, we need to introduce a few definitions and notations. For more information, we direct the readers to the work of Otunuga et al. $[16,17]$.

For each $i \in I\left(1, K^{*}\right)$, let $\tau_{i-1}$ and $\bar{\gamma}_{i-1}$ be finite constant time delays such that $0<\bar{\gamma}_{i-1} \leq \tau_{i-1}$. If $K=0$, then there is no jump and we simply write $\tau_{i-1}=\tau$ and $\bar{\gamma}_{i-1}=\bar{\gamma}$. Here, $\tau_{i-1}$ characterizes the influence of the past performance history of state of dynamic process. $\bar{\gamma}_{i-1}$ describes the reaction or response time delays. In general, these time delays are unknown and random variables. These types of delays play an important role in developing mathematical models of continuous time [30] and discrete-time $[22,31]$ dynamic 
processes. Based on the practical nature of data collection process, it is essential to either transform these time delays into positive integers or design a suitable data collection schedule or discretization process. For this purpose, for each $i \in I\left(1, K^{*}\right)$, we describe the discrete version of time delays of $\tau_{i}$ and $\gamma_{i}$ as follows:

$$
\begin{aligned}
\left\{r_{i-1}\right. & =\left[\left|\frac{\tau_{i-1}}{\Delta t_{i-1}}\right|\right]+1, \\
\overline{\bar{\gamma}}_{i-1} & =\left[\left|\frac{\bar{\gamma}_{i-1}}{\Delta t_{i-1}}\right|\right]+1, \text { for } i \in I\left(1, K^{*}\right) .
\end{aligned}
$$

Here, $[||$.$] is the integer part of a real number, and for the$ sake of simplicity, we assume that $0<\bar{\gamma}_{i-1}<1,\left(\overline{\bar{\gamma}}_{i-1}=1\right)$.

Definition 6. Let $\mathbf{x}=\left[x_{1}, x_{2}, \cdots, x_{n}\right]^{T}$ be a continuous time multivariate stochastic process defined on an interval $\left[t_{0}-\tau, T\right]$ into $\mathfrak{R}^{n}$, for some $T>0$. For $t \in\left[t_{0}-\tau, T\right]$, let $\mathscr{F}_{t}$ be an increasing subsigma algebra of a complete probability space $(\Omega, \mathscr{F}, \mathscr{P})$ for which $\mathbf{x}(t)$ is $\mathscr{F}_{t}$ measurable. For each $i \in I\left(1, K^{*}\right)$, let $\mathbb{P}$ and $\mathbb{P}^{i-1}$ be a partition of $\left[t_{0}-\tau, T\right]$ and $\left[T_{i-1}-\tau_{i-1}, T_{i}\right]$, respectively. The partition $\mathbb{P}^{i-1}$ is derived by decomposing the partition $\mathbb{P}$. For each $i \in I\left(1, K^{*}\right)$, the partitions $\mathbb{P}$ and $\mathbb{P}^{i-1}$ are defined as follows:

$$
\left\{\begin{array}{l}
\mathbb{P}=\left\{t_{k}: t_{k}=t_{0}+k \Delta t, \quad k \in I(-r, N)\right\}, \\
\mathbb{P}^{i-1}=\left\{t_{k}^{i-1}: t_{k}^{i-1}=T_{i-1}+k \Delta t, k \in I\left(-r_{i-1}, N_{i-1}\right)\right\},
\end{array}\right.
$$

where $t_{k}^{i-1}$ represents time $t_{k}$ in the interval $\left[T_{i-1}-\tau_{i-1}, T_{i}\right]$, $\Delta t=\left(T-t_{0}\right) / N \equiv\left(T_{i}-T_{i-1}\right) / N_{i-1}$, and $T_{0}=t_{0}$.

Remark 7. We define $\mathcal{S}_{i}=\sum_{l=1}^{i} N_{l-1}$ with $\mathcal{S}_{0}=0$. For $K \neq 0$, we note that we can write $\mathbb{P}$ as $\left\{t_{0}<t_{1}<\cdots<t_{N_{0}}<t_{N_{0}+1}<\cdots\right.$. $\left.<t_{N_{0}+N_{1}}<t_{N_{0}+N_{1}+1}<\cdots<t_{\mathcal{S}_{i-1}}<t_{\delta_{i-1}+1}<\cdots<t_{\mathcal{S}_{i}}<\cdots<T\right\}$. From this, it follows directly that the jump times $T_{i}$ are contained in $\mathbb{P}$. For any $t_{k}^{i-1} \in \mathbb{P}^{i-1}, k \in\left[0, N_{i-1}\right]$, we have $t_{k}^{i-1} \in \mathbb{P}$. Hence, there is a relationship between elements of $\mathbb{P}^{i-1}$ with $\mathbb{P}$ that is described by $t_{k}^{i-1}=t_{\mathcal{S}_{i-1}+k}$, for $k \in I\left(0, N_{i}\right)$. In fact, the relationship between a set of jump times $\left\{T_{1}, T_{2}, \cdots, T_{K}\right\}$ and the partition $\mathbb{P}$ defined in (40) is as $T_{i} \rightarrow t_{\mathcal{S}_{i}}$, where $N_{i-1}$ is the size of partition $\mathbb{P}^{i-1}$ of the subinterval $\left[T_{i-1}, T_{i}\right]$. It follows that $\mathcal{S}_{K^{*}}=N$. Using these facts, and noting that if $K=0$, then $\mathbb{P}^{i-1}=\mathbb{P}, N_{i-1}=N, \tau_{i-1}=\tau, \gamma_{i-1}=\gamma, r_{i-1}=r$, $\eta_{i-1}=\eta, t_{k}^{i-1}=t_{k}$ and (40) reduces to

$$
\mathbb{P}^{i-1}=\left\{t_{k}^{i-1}: t_{k}^{i-1}=T_{i-1}+k \Delta t, k \in I\left(-r_{i-1}, N_{i-1}\right)\right\} .
$$

For each $i \in I\left(1, K^{*}\right)$, let $\left\{\mathbf{x}^{i-1}\left(t_{k}^{i-1}\right)\right\}_{k=-r_{i-1}}^{N_{i-1}}$ be a finite sequence corresponding to the stochastic process $x$ and partition $\mathbb{P}^{i-1}$ defined in (41). We simply write $\mathbf{x}\left(t_{k}^{i-1}\right) \equiv \mathbf{x}^{i-1}$ $\left(t_{k}^{i-1}\right)$. We further recall that $x\left(t_{k}^{i-1}\right)$ is $\mathscr{F}_{t_{k}^{i-1}}$ measurable for $k \in I\left(-r_{i-1}, N_{i-1}\right)$. We also recall the definition of forward time shift operator $F[16,17,32]$ :

$$
F^{l} \mathbf{x}\left(t_{k}^{i-1}\right)=\mathbf{x}\left(t_{k+l}^{i-1}\right), l \geq 0
$$

Definition 8. For $q_{i-1}=1$ and $r_{i-1} \geq 1$, each $k \in I\left(0, N_{i-1}\right)$ and each $m_{k}^{i-1} \in I\left(2, r_{i-1}+\mathcal{S}_{i-1}+k-1\right)$, a partition $P_{k}^{i-1}$ of closed interval $\left[t_{k-m_{k}^{i-1}}^{i-1}, t_{k-1}^{i-1}\right]$ is called local at time $t_{k}^{i-1}$, and it is defined by

$$
P_{k}^{i-1}:=t_{k-m_{k}^{i-1}}^{i-1}<t_{k-m_{k}^{i-1}+1}^{i-1}<\cdots<t_{k-1}^{i-1} .
$$

Moreover, $P_{k}^{i-1}$ is referred to as the $m_{k}^{i-1}$ point subpartition of the partition $\mathbb{P}^{i-1}$ in (41) of the closed subinterval $\left[t_{k-m_{k}^{i-1}}^{i-1}, t_{k-1}^{i-1}\right]$ of $\left[-\tau_{i-1}, T_{i}\right]$.

Remark 9. We note that for $K=0$, that is, no jump, we have $P_{k}^{i-1}=P_{k}, m_{k}^{i-1}=m_{k}, t_{\mathrm{k}-m_{k}^{i-1}}^{i-1}=t_{k-m_{k}}^{i-1}$, and $t_{k-1}^{i-1}=t_{k-1}$, where $P_{k}$ is referred as the $m_{k}$ point subpartition of partition $\mathbb{P}$ in (40) of the closed subinterval $\left[t_{k-m_{k}}, t_{k}\right]$ of $\left[t_{0}-\tau, T\right]$ for $k \in$ $I(0, N)$.

Definition 10. For each $i \in I\left(1, K^{*}\right), k \in I\left(0, N_{i-1}\right)$, and $m_{k}^{i-1}$ $\in I\left(2, r_{i-1}+\mathcal{S}_{i-1}+k-1\right)$, a local finite sequence $\mathbf{S}_{m_{k}^{i-1}, k}$ at $t_{k}^{i-1}$ of size $m_{k}^{i-1}$ is the restriction [33] of $\left\{\mathbf{x}\left(t_{k}^{i-1}\right)\right\}_{k=-r_{i-1}}^{N i-1}$ to $P_{k}^{i-1}$ in (43). This restriction sequence is defined by

$$
\mathbf{S}_{m_{k}^{i-1}, k}:=\left\{F^{l} \mathbf{x}\left(t_{k-1}^{i-1}\right)\right\}_{l=-m_{k}^{i-1}+1}^{0} .
$$

As $m_{k}^{i-1}$ varies from 2 to $r_{i-1}+\mathcal{S}_{i-1}+k-1$, the corresponding respective local sequence $\mathbf{S}_{m_{k}^{i-1}, k}$ at $t_{k}^{i-1}$ varies from $\left\{\mathbf{x}\left(t_{l}^{i-1}\right)\right\}_{l=k-2}^{k-1}$ to $\left\{\mathbf{x}\left(t_{l}^{i-1}\right)\right\}_{l=-r_{i-1}+\mathcal{S}_{i-1}+1}^{k-1}$. As a result of this, the sequence defined in (44) is also called a $m_{k}^{i-1}$ local moving sequence. Furthermore, the average corresponding to the local sequence $S_{m_{k}^{i-1}, k}$ in (44) is defined by

$$
\overline{\mathbf{S}}_{m_{k}^{i-1}, k}=\frac{1}{m_{k}^{i-1}} \sum_{l=-m_{k}^{i-1}+1}^{0} F^{l} \mathbf{x}\left(t_{k-1}^{i-1}\right) .
$$

The average/mean defined in (45) is also called the $m_{k}^{i-1}$ local average/mean. For $i \in I\left(1, K^{*}\right)$ and $k \in I\left(0, N_{i-1}\right)$, the $m_{k}^{i-1}$ local covariance matrix corresponding to the local sequence $\mathbf{S}_{m_{k}^{i-1}, k}$ in (44) is defined by

$$
\sum_{m_{k}^{i-1}, k}=\left(\begin{array}{ccccc}
s_{m_{k}^{i-1}, k}^{1,1} & s_{m_{k}^{i-1}, k}^{1,2} & s_{m_{k}^{i-1}, k}^{1,3} & \cdots & s_{m_{k}^{i-1}, k}^{1, n} \\
s_{m_{k}^{i-1}, k}^{2,1} & s_{m_{k}^{i-1}, k}^{2,2} & s_{m_{k}^{i-1}, k}^{2,3} & \cdots & s_{m_{k}^{i-1}, k}^{2, n} \\
\vdots & \ddots & \ddots & \ddots & \vdots \\
s_{m_{k}^{i-1}, k}^{n, 1} & s_{m_{k}^{i-1}, k}^{n, 2} & s_{m_{k}^{n-1}, k}^{n, 3} & \cdots & s_{m_{k}^{i-1}, k}^{n, n}
\end{array}\right)
$$


where $s_{m_{k}^{i-1}, k}^{j, l} \equiv s_{m_{k}^{i-1}, k}^{j, l}(x), j, l \in I(1, n)$ is the local sample covariance statistic between $x_{j}$ and $x_{l}$ at $t_{k}^{i-1}$ described by

$s_{m_{k}^{j, l}, k}^{i-1}:=\left\{\begin{array}{l}\frac{1}{m_{k}^{i-1}} \sum_{a=-m_{k}^{i-1}+1}^{0}\left(F^{a} x_{j}\left(t_{k-1}^{i-1}\right)-\frac{1}{m_{k}^{i-1}} \sum_{b=-m_{k}^{i-1}+1}^{0} F^{b} x_{j}\left(t_{k-1}^{i-1}\right)\right)\left(F^{a} x_{l}\left(t_{k-1}^{i-1}\right)-\frac{1}{m_{k}^{i-1}} \sum_{b=-m_{k}^{i-1}+1}^{0} F^{b} x_{l}\left(t_{k-1}^{i-1}\right)\right), \text { for small } m_{k}^{i-1}, \\ \frac{1}{m_{k}^{i-1}-1} \sum_{a=-m_{k}^{i-1}+1}^{0}\left(F^{a} x_{j}\left(t_{k-1}^{i-1}\right)-\frac{1}{m_{k}^{i-1}} \sum_{b=-m_{k}^{i-1}+1}^{0} F^{b} x_{j}\left(t_{k-1}^{i-1}\right)\right)\left(F^{a} x_{l}\left(t_{k-1}^{i-1}\right)-\frac{1}{m_{k}^{i-1}} \sum_{b=-m_{k}^{i-1}+1}^{0} F^{b} x_{l}\left(t_{k-1}^{i-1}\right)\right), \text { for large } m_{k}^{i-1} .\end{array}\right.$

Denoting $\mathbf{x}(k) \equiv \mathbf{x}\left(t_{k}^{i-1}\right)$ for $i \in I\left(1, K^{*}\right)$ and $k \in I\left(1, N_{i-1}\right)$, the discrete-time interconnected dynamic model of local sample mean $\bar{S}_{m_{k}, k}$ and variance $s_{m_{k}, k}^{2}$ processes (DTIDMLSMVSP) $[16,17]$ is given by

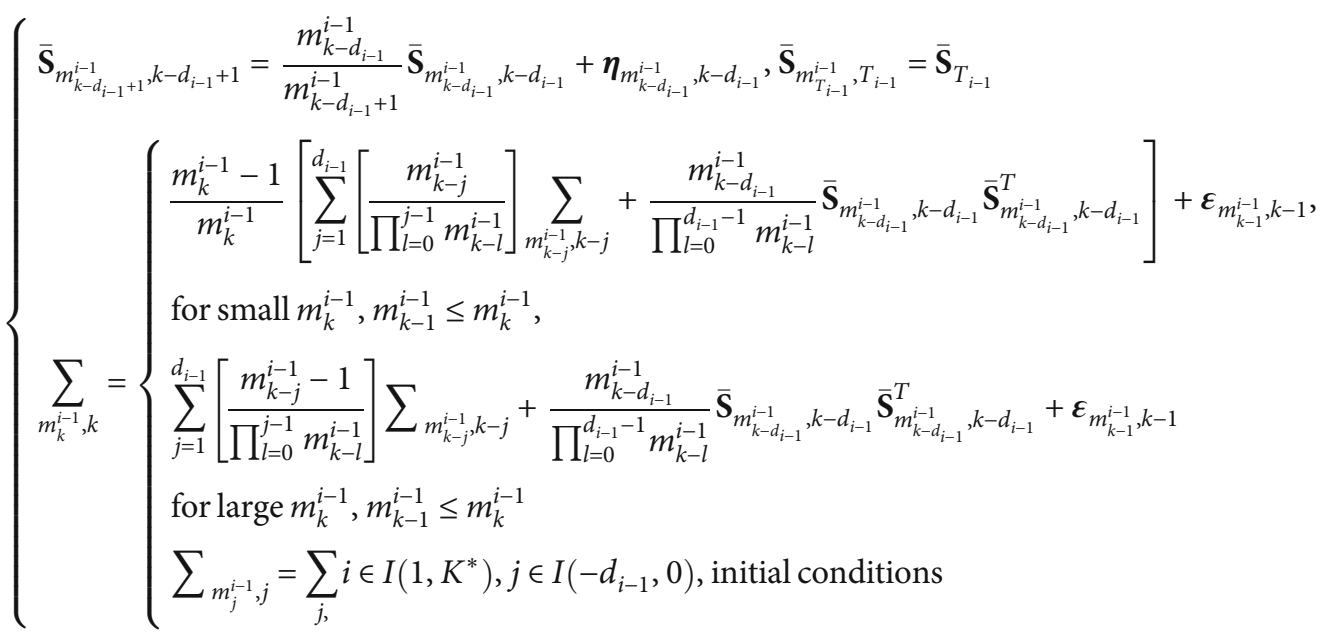


where $d_{i-1}$ is the order of the system and

$$
\begin{aligned}
& \boldsymbol{\eta}=\left(\begin{array}{c}
\eta^{1} \\
\eta^{2} \\
\vdots \\
\eta^{n}
\end{array}\right), \\
& \varepsilon_{m_{k}^{i-1}, k}=\left(\begin{array}{ccccc}
\varepsilon_{m_{k}^{i-1}, k}^{1,1} & \varepsilon_{m_{k}^{i-1, k}}^{1,2} & \varepsilon_{m_{k}^{1-1}, k}^{1,3} & \cdots & \varepsilon_{m_{k}^{1-1}, k}^{1, n} \\
\varepsilon_{m_{k}^{i-1}, k}^{2,1} & \varepsilon_{m_{k}^{i-1, k}, k}^{2,2} & \varepsilon_{m_{k}^{2 i-1}, k}^{2,3} & \cdots & \varepsilon_{m_{k}^{2 i-1}, k}^{2, n} \\
\vdots & \ddots & \ddots & \ddots & \vdots \\
\varepsilon_{m_{k}^{i-1}, k}^{n, 1} & \varepsilon_{m_{k}^{i-1}, k}^{n, 2} & \varepsilon_{m_{k}^{i-1}, k}^{n, 3} & \cdots & \varepsilon_{m_{k}^{i-1}, k}^{n, n}
\end{array}\right) \text {, } \\
& \boldsymbol{\epsilon}_{m_{k}^{i-1}, k}=\left(\begin{array}{ccccc}
\varepsilon_{m_{k}^{i-1}, k}^{1,1} & \varepsilon_{m_{k}^{i-1}, k}^{1,2} & \varepsilon_{m_{k}^{i-1}, k}^{1,3} & \cdots & \varepsilon_{m_{k}^{i-1}, k}^{1, n} \\
\varepsilon_{m_{k}^{i-1}, k}^{2,1} & \varepsilon_{m_{k}^{i-1, k}}^{2,2} & \varepsilon_{m_{k}^{i-1, k}}^{2,3} & \cdots & \varepsilon_{m_{k}^{i-1}, k}^{2, n} \\
\vdots & \ddots & \ddots & \ddots & \vdots \\
\varepsilon_{m_{k}^{i-1}, k}^{n, 1} & \varepsilon_{m_{k}^{i-1}, k}^{n, 2} & \varepsilon_{m_{k}^{i-1}, k}^{n, 3} & \cdots & \varepsilon_{m_{k}^{i-1}, k}^{n, n}
\end{array}\right) \\
& \left\{\begin{array}{l}
\eta_{m_{k-d_{i-1}}^{j}, k-d_{i-1}}^{j}=\frac{1}{m_{k-d_{i-1}+1}^{i-1}+1}\left[\sum_{l=-m_{k-d_{i-1}+1}^{i-1}+1}^{-m_{k-d_{i-1}}^{i-1}+1} F^{l} x_{j}\left(k-d_{i-1}\right)-F^{-m_{k-d_{i-1}}^{i-1}+1} x_{j}\left(k-d_{i-1}\right)-F^{-m_{k-d_{i-1}}^{i-1}} x_{j}\left(k-d_{i-1}\right)+F^{0} x_{j}\left(k-d_{i-1}\right)\right] \\
\varepsilon_{m_{k-1}^{i-1}, k-1}^{j, l}=\frac{m_{k}^{i-1}-1}{m_{k}^{i-1}}\left[\sum_{l=1}^{d_{i-1}} \frac{F^{-l+1} x_{j}(k-1) F^{-l+1} x_{l}(k-1)}{\prod_{a=0}^{l-1} m_{k-a}^{i-1}}-\sum_{l=1}^{d_{i-1}} \frac{F^{-l+1-m_{k-l}^{i-1}} x_{j}(k-1) F^{-l+1-m_{k-l}^{i-1}} x_{l}(k-1)}{\prod_{a=0}^{l-1} m_{k-a}^{i-1}}-\sum_{l=1}^{d_{i-1}} \frac{F^{-l+2-m_{k-1}^{i-1}} x_{j}(k-1) F^{-l+2-m_{k-1}^{i-1}} x_{l}(k-1)}{\prod_{a=0}^{l-1} m_{k-a}^{i-1}}\right]
\end{array}\right. \\
& +\frac{m_{k}^{i-1}-1}{m_{k}^{i-1}}\left[\sum_{l=1}^{d_{i-1}}\left[\frac{\sum_{v=-l+2-m_{k-1+1}^{i-1}}^{-l+2-m_{j}^{i-1}} F^{v} x_{j}(k-1) F^{v} x_{l}(k-1)}{\prod_{a=0}^{l-1} m_{k-a}^{i-1}}\right]+\sum_{i=1}^{d_{i-1}}\left[\frac{\sum_{v, s=-l+2-m_{k-l+1}^{i-1} v \neq s}^{-l+1} F^{v} x_{j}(k-1) F^{s} x_{l}(k-1)}{\prod_{a=0}^{l-1} m_{k-a}^{i-1}}\right]\right] \\
& -\frac{1}{m_{k}^{i-1}} \sum_{v, s=-m_{k}^{i-1}+1 v \neq s}^{0} F^{v} x_{j}(k-1) F^{s} x_{l}(k-1) \text {, } \\
& \epsilon_{m_{k-1}^{i-1}, k-1}^{j, l}=\sum_{l=1}^{d_{i-1}} \frac{F^{-l+1} x_{j}(k-1) F^{-l+1} x_{l}(k-1)}{\prod_{a=0}^{l-1} m_{k-a}^{i-1}}-\sum_{l=1}^{d_{i-1}} \frac{F^{-l+1-m_{k-l}^{i-1}} x_{j}(k-1) F^{-l+1-m_{k-1}^{i-1}} x_{l}(k-1)}{\prod_{a=0}^{l-1} m_{k-a}^{i-1}}-\sum_{i=1}^{d_{i-1}} \frac{F^{-l+2-m_{k-1}^{i-1}} x_{j}(k-1) F^{-l+2-m_{k-1}^{i-1}} x_{l}(k-1)}{\prod_{a=0}^{l-1} m_{k-a}^{i-1}}
\end{aligned}
$$

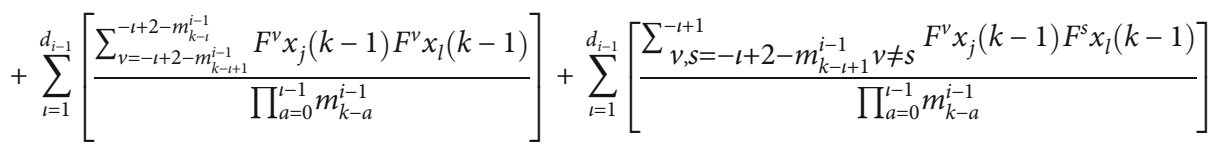

$$
\begin{aligned}
& -\frac{1}{m_{k}^{i-1}-1} \sum_{v, s=-m_{k}^{i-1}+1 v \neq s}^{0} F^{v} x_{j}(k-1) F^{s} x_{l}(k-1) \text {. }
\end{aligned}
$$

The proof of this is given in [16].

\section{Parametric Estimation}

In this section, we consider a parameter estimation problem in drift and diffusion coefficients of (34). This is achieved by utilizing the lagged adaptive process [22] and the interconnected discrete-time dynamics of local sample mean and variances statistic processes model (48). For each $i \in I\left(1, K^{*}\right)$, we consider a general interconnected hybrid system described by the system of stochastic differential equations:

$$
\left\{\begin{array}{l}
d \mathbf{x}^{i-1}=\mathbf{f}^{i-1}(t, \mathbf{x}) d t+\boldsymbol{\sigma}^{i-1}(t, \mathbf{x}) d \mathbf{W}(t), \mathbf{x}\left(T_{i-1}\right)=\mathbf{x}^{i-1}, t \in\left[T_{i-1}, T_{i}\right), \\
\mathbf{x}^{i}=\Gamma^{i} \mathbf{x}^{i-1}\left(T_{i}^{-}, T_{i-1}, \mathbf{x}^{i-1}\right)
\end{array}\right.
$$


where $\Gamma^{i}=\operatorname{diag}\left(\Gamma_{1}^{i}, \Gamma_{2}^{i}, \cdots, \Gamma_{j}^{i}, \cdots, \Gamma_{n}^{i}\right)$ is the jump coefficient matrix; the jump times $T_{i}$ 's are defined in (34). For each $j \in I(1, n)$, the estimate of the jump coefficient $\Gamma_{j}^{i}$ is given by $\Gamma_{j}^{i}=x_{j}\left(T_{i}\right) / \lim _{t \rightarrow T_{i}^{-}} x_{j}^{i-1}\left(t, T_{i-1}, x^{i-1}\right)$.
Let $V \in C\left[[-\tau, \infty] \times \Re^{n}, \Re^{m}\right]$, and its partial derivatives $V_{t}, \partial V / \partial x$, and $\partial^{2} V / \partial x^{2}$ exist and are continuous on each interval $\left[T_{i-1}, T_{i}\right]$. We apply Itô-Doob stochastic differential formula [34] to $V$, and we obtain

$$
\left\{\begin{array}{l}
d V\left(t, \mathbf{x}^{i-1}\right)=\mathbf{L} V\left(t, \mathbf{x}^{i-1}\right) d t+V_{\mathbf{x}}\left(t, \mathbf{x}^{i-1}\right) \sigma\left(t, \mathbf{x}^{i-1}\right) d W(t), \mathbf{x}\left(T_{i-1}\right)=\mathbf{x}^{i-1}, t \in\left[T_{i-1}, T_{i}\right), \\
V\left(T_{i}, \mathbf{x}^{i}\right)=V\left(T_{i}, \Gamma^{i} \mathbf{x}^{i-1}\left(T_{i}^{-}, T_{i-1}, \mathbf{x}^{i-1}\right)\right) .
\end{array}\right.
$$

where the $L$ operator is defined by

$$
\left\{\begin{array}{l}
\mathbf{L} V\left(t, x^{i-1}\right)=V_{t}\left(t, \mathbf{x}^{i-1}\right)+V_{x}\left(t, \mathbf{x}^{i-1}\right) f\left(t, \mathbf{x}^{i-1}\right)+\frac{1}{2} \operatorname{tr}\left(V_{x x}\left(t, \mathbf{x}^{i-1}\right)\right) c\left(t, \mathbf{x}^{i-1}\right), \\
c\left(t, \mathbf{x}^{i-1}\right)=\boldsymbol{\sigma}^{i-1}\left(t, \mathbf{x}^{i-1}\right) \boldsymbol{\sigma}^{i-1}\left(t, \mathbf{x}^{i-1}\right)^{\top} .
\end{array}\right.
$$

For (50) and (51), we present the Euler-type discretization scheme [23]:

$$
\left\{\begin{array}{l}
\Delta \mathbf{x}^{i-1}\left(t_{k}^{i-1}\right)=\mathbf{f}\left(t_{k-1}^{i-1}, \mathbf{x}\left(t_{k-1}^{i-1}\right)\right) \Delta t_{k}^{i-1}+\boldsymbol{\sigma}^{i-1}\left(t_{k-1}^{i-1}, \mathbf{x}\left(t_{k-1}^{i-1}\right)\right) \Delta \mathbf{W}\left(t_{k}^{i-1}\right), k \in I\left(1, N_{i-1}\right), \\
\mathbf{x}^{i}=\Gamma^{i} \mathbf{x}^{i-1}\left(T_{i}^{-}, T_{i-1}, \mathbf{x}^{i-1}\right), \\
\Delta V\left(t_{k}^{i-1}, \mathbf{x}^{i-1}\left(t_{k}^{i-1}\right)\right)=L V\left(t_{k-1}^{i-1}, \mathbf{x}^{i-1}\left(t_{k-1}^{i-1}\right)\right) \Delta t_{k}^{i-1}+V_{x}\left(t_{k-1}^{i-1}, \mathbf{x}^{i-1}\left(t_{k-1}^{i-1}\right)\right) \boldsymbol{\sigma}^{i-1}\left(t_{k-1}^{i-1}, \mathbf{x}\left(t_{k-1}^{i-1}\right)\right) \Delta W\left(t_{k}^{i-1}\right), \\
V\left(T_{i}, \mathbf{x}^{i}\right)=V\left(T_{i}, \Gamma^{i} \mathbf{x}^{i-1}\left(T_{i}^{-}, T_{i-1}, \mathbf{x}^{i-1}\right)\right) .
\end{array}\right.
$$

Define $\mathscr{F}_{t_{k-1}}^{i-1} \equiv \mathscr{F}_{k-1}^{i-1}$ as the filtration process up to time $t_{k-1}^{i-1}$. With regard to the continuous time dynamic system
(50) and its transformed system (51), the more general moments of $\Delta x\left(t_{k}^{i-1}\right)$ are as follows:

$$
\left\{\begin{array}{l}
\mathbb{E}\left[\Delta \mathbf{x}\left(t_{k}^{i-1}\right) \mid \mathscr{F}_{k-1}^{i-1}\right]=\mathbf{f}^{i-1}\left(t_{k-1}^{i-1}, \mathbf{x}^{i-1}\left(t_{k-1}^{i-1}\right)\right) \Delta t_{k}^{i-1}, \\
\mathbb{E}\left[\left(\Delta x^{i-1}\left(t_{k}^{i-1}\right)-\mathbb{E}\left[\Delta \mathbf{x}^{i-1}\left(t_{k}^{i-1}\right) \mid \mathscr{F}_{k-1}^{i-1}\right]\right)\left(\Delta \mathbf{x}^{i-1}\left(t_{k}^{i-1}\right)-\mathbb{E}\left[\Delta \mathbf{x}^{i-1}\left(t_{k}^{i-1}\right) \mid \mathscr{F}_{k-1}^{i-1}\right]\right)^{T} \mid \mathscr{F}_{k-1}^{i-1}\right]=\sigma^{i-1}\left(t_{k-1}^{i-1}, \mathbf{x}^{i-1}\left(t_{k-1}^{i-1}\right)\right) \boldsymbol{\sigma}^{i-1}\left(t_{k-1}^{i-1}, \mathbf{x}^{i-1}\left(t_{k-1}^{i-1}\right)\right)^{T} \Delta t_{k}^{i-1}, \\
\mathbb{E}\left[\Delta V\left(t_{k}^{i-1}, \mathbf{x}^{i-1}\left(t_{k}^{i-1}\right)\right) \mid \mathscr{F}_{k-1}^{i-1}\right]=L V\left(t_{k-1}^{i-1}, \mathbf{x}^{i-1}\left(t_{k}^{i-1}\right),\right) \Delta t_{k}^{i-1}, \\
\mathbb{E}\left[\left(\Delta V\left(t_{k}^{i-1}, \mathbf{x}^{i-1}\left(t_{k}^{i-1}\right)\right)-\mathbb{E}\left[\Delta V\left(t_{k}^{i-1}, \mathbf{x}^{i-1}\left(t_{k}^{i-1}\right)\right) \mid \mathscr{F}_{k-1}^{i-1}\right]\right)\left(\Delta V\left(t_{k}^{i-1}, \mathbf{x}^{i-1}\left(t_{k}^{i-1}\right)\right)-\mathbb{E}\left[\Delta V\left(t_{k}^{i-1}, \mathbf{x}^{i-1}\left(t_{k}^{i-1}\right)\right) \mid \mathscr{F}_{k-1}^{i-1}\right]\right)^{T} \mid \mathscr{F}_{k-1}^{i-1}\right]=B\left(t_{k-1}^{i-1}, \mathbf{x}^{i-1}\left(t_{k-1}^{i-1}\right)\right),
\end{array}\right.
$$


where $B\left(t_{k-1}^{i-1}, \mathbf{x}\left(t_{k-1}^{i-1}\right)\right)=V_{x}\left(t_{k-1}^{i-1}, \mathbf{x}^{i-1}\left(t_{k-1}^{i-1}\right)\right) c\left(t_{k-1}^{i-1}, \mathbf{x}\left(t_{k-1}^{i-1}\right)\right) V_{x}$ $\left(t_{k-1}^{i-1}, \mathbf{x}\left(t_{k-1}^{i-1}\right)\right)^{\top} \Delta t_{k}^{i-1}$, and $T$ stands for the transpose of the matrix. From (53) and (54), we have

$$
\left\{\begin{array}{l}
\Delta \mathbf{x}^{i-1}\left(t_{k}^{i-1}\right)=\mathbb{E}\left[\Delta \mathbf{x}^{i-1}\left(t_{k}^{i-1}\right) \mid \mathscr{F}_{k-1}^{i-1}\right]+\boldsymbol{\sigma}^{i-1}\left(t_{k-1}^{i-1}, \mathbf{x}^{i-1}\left(t_{k-1}^{i-1}\right)\right) \Delta \mathbf{W}\left(t_{k}^{i-1}\right), k \in I\left(1, N_{i-1}\right), \\
\Delta V\left(t_{k}^{i-1}, \mathbf{x}^{i-1}\left(t_{k}^{i-1}\right)\right)=\mathbb{E}\left[\Delta V\left(t_{k}^{i-1}, \mathbf{x}^{i-1}\left(t_{k}^{i-1}\right)\right) \mid \mathscr{F}_{k-1}^{i-1}\right]+V_{\mathbf{x}}^{i-1}\left(t_{k-1}^{i-1}, \mathbf{x}^{i-1}\left(t_{k-1}^{i-1}\right)\right) \boldsymbol{\sigma}^{i-1}\left(t_{k-1}^{i-1}, \mathbf{x}^{i-1}\left(t_{k-1}^{i-1}\right)\right) \Delta W\left(t_{k}\right) .
\end{array}\right.
$$

This provides the basis for the development of the concept of lagged adaptive expectation process [22] with respect to continuous time stochastic dynamic system (51). This indeed leads to a formulation of $m_{k}^{i-1}$ local generalized method of moments at $t_{k}^{i-1}$.

In the following, we state a result that exhibits the existence of the solution of the system of nonlinear algebraic equations. For the sake of easy reference, we shall state the implicit function theorem without proof.

Theorem 11. Implicit Function Theorem [33]. Let $\boldsymbol{F}=\left\{F_{1}\right.$, $\left.F_{2}, \cdots, F_{q}\right\}$ be a vector-valued function defined on an open set $S \in \mathfrak{R}^{q+k}$ with values in $\mathfrak{R}^{q}$. Suppose $\boldsymbol{F} \in C^{\prime}$ on $S$. Let $\left(\boldsymbol{u}_{0} ; \boldsymbol{v}_{0}\right)$ be a point in $S$ for which $\boldsymbol{F}\left(\boldsymbol{u}_{0} ; \boldsymbol{v}_{0}\right)=0$ and for which the $q \times q$ determinant of the Jacobian matrix det $\left[\boldsymbol{J}_{\boldsymbol{F}}\left(\boldsymbol{v}_{0}\right)\right] \neq 0$. Then, there exists a $k$-dimensional open set $\boldsymbol{T}_{0}$ containing $\boldsymbol{v}_{0}$ and unique vector-valued function $\boldsymbol{g}$, defined on $\boldsymbol{T}_{0}$ and having values in $\mathfrak{R}^{q}$, such that $\boldsymbol{g} \in$ $C^{\prime}$ on $\boldsymbol{T}_{0}, \boldsymbol{g}\left(\boldsymbol{v}_{0}\right)=\boldsymbol{u}_{0}$, and $\boldsymbol{F}(\boldsymbol{g}(\boldsymbol{v}) ; \boldsymbol{v})=0$ for every $\boldsymbol{v} \in$ $\mathrm{T}_{0}$.

6.1. Special Case: Illustration. For each $j, l \in I(1, n)$ and each $i \in \boldsymbol{I}\left(1, K^{*}\right)$, we consider a special case of (14).

$$
\left\{\begin{array}{l}
d y_{j}=\left(u_{j}^{i-1}-y_{j}\right)\left[\kappa_{j, j}^{i-1} y_{j}+\sum_{l \neq j}^{n} \kappa_{j, l}^{i-1} y_{l}\right] d t+\delta_{j, j}^{i-1}\left(u_{j}^{i-1}-y_{j}\right) d W_{j, j}(t)+\left(u_{j}^{i-1}-y_{j}\right) \sum_{l \neq j}^{n} \delta_{j, l}^{i-1} y_{l} d W_{j, l}(t), y_{j}\left(T_{i-1}\right)=y_{j}^{i-1}, t \in\left[T_{i-1}, T_{i}\right), \\
y_{j}^{i}=\pi_{j}^{i} y_{j}^{i-1}\left(T_{i}^{-}, T_{i-1}, y^{i-1}\right), \\
d p_{j}(t)=p_{j}\left[\gamma_{j, j}^{i-1}\left(y_{j}-p_{j}\right)+\beta_{j}^{i-1}+\sum_{l \neq j}^{n} \gamma_{j, l}^{i-1} p_{l}(t)\right] d t+\sigma_{j, j}^{i-1} p_{j} d Z_{j, j}(t)+p_{j} \sum_{l \neq j}^{n} \sigma_{j, l}^{i-1} p_{l} d Z_{j, l}(t), p_{j}\left(T_{i-1}\right)=p_{j}^{i-1}, t \in\left[T_{i-1}, T_{i}\right), \\
p_{j}^{i}=\theta_{j}^{i} p_{j}^{i-1}\left(T_{i}^{-}, T_{i-1}, \boldsymbol{y}^{i-1}, \boldsymbol{p}^{i-1}\right) .
\end{array}\right.
$$

Here, $\kappa_{j, l}^{i-1}, u_{j}^{i-1}, \beta_{j}^{i-1}, \gamma_{j, l}^{i-1}, \delta_{j, l}^{i-1}$, and $\sigma_{j, l}^{i-1}$ are the system parameters on the jump subinterval $\left[T_{i-1}, T_{i}\right) ; u_{j}^{i-1}$, $\kappa_{j, j}^{i-1}, \gamma_{j, j}^{i-1}, \delta_{j, j}^{i-1}$, and $\sigma_{j, j}^{i-1}$ are positive; and for $l \neq j, \kappa_{j, l}^{i-1}$, $\gamma_{j, l}^{i-1} \in \Re ; \delta_{j, l}^{i-1}, \sigma_{j, l}^{i-1}$ are nonnegative. $W$ and $Z$ are independent standard Wiener process on a filtered probability space $\left(\Omega, \mathscr{F},\left(\mathscr{F}_{t}\right)_{t \geq 0}, \mathscr{P}\right)$ with the properties described in (14). It follows that the interconnected system of stochastic differential equations (56) has $4 n^{2}+2 n$ parameters. Also,

$$
\left\{\kappa_{j, l}\right\}_{l \neq j}^{i-1}\left\{\begin{array}{l}
>0 \text { if } y_{l} \text { is cooperating with } y_{j}, \\
<0 \text { if } y_{l} \text { is competing with } y_{j}, \\
=0 \text { if there is no interaction between } y_{l} \text { and } y_{j}, j, l \in I(1, n)
\end{array}\right.
$$


and likewise,

$\left\{\gamma_{j, l}\right\}_{l \neq j}^{i-1}\left\{\begin{array}{l}>0 \text { if } p_{l} \text { is cooperating with } p_{j}, \\ <0 \text { if } p_{l} \text { is competing with } p_{j}, \\ =0 \text { if there is no interaction between } p_{l} \text { and } p_{j}, j, l \in I(1, n) .\end{array}\right.$

Remark 12. For the case $K=0,(56)$ is reduced to

$$
\left\{\begin{array}{l}
d \mathrm{y}_{j}=\left(u_{j}-y_{j}\right)\left[\kappa_{j, j} y_{j}+\sum_{l \neq j}^{n} \kappa_{j, l} y_{l}\right] d t+\delta_{j, j}\left(u_{j}-y_{j}\right) d W_{j, j}(t)+\left(u_{j}-y_{j}\right) \sum_{l \neq j}^{n} \delta_{j, l} y_{l} d W_{j, l}(t), y_{j}\left(t_{0}\right)=y_{j 0}, t \in\left[t_{0}, T\right], \\
d p_{j}(t)=p_{j}\left[\gamma_{j, j}\left(y_{j}-p_{j}\right)+\beta_{j}+\sum_{l \neq j}^{n} \gamma_{j, l} p_{l}(t)\right] d t+\sigma_{j, j} p_{j} d Z_{j, j}(t)+p_{j} \sum_{l \neq j}^{n} \sigma_{j, l} p_{l} d Z_{j, l}(t), p_{j}\left(t_{0}\right)=p_{j 0}, t \in\left[t_{0}, T\right],
\end{array}\right.
$$

where for $j, l \in I(1, n)$, the parameters $\kappa_{j, l}, u_{j}, \beta_{j}, \gamma_{j, l}, \delta_{j, l}$, and $\sigma_{j, l}$ are the system parameters on the interval $\left[t_{0}, T\right] ; u_{j}, \kappa_{j, j}$, $\gamma_{j, j}, \delta_{j, j}$, and $\sigma_{j, j}$ are positive; and for $l \neq j, \kappa_{j, l}, \gamma_{j, l} \in \Re ; \delta_{j, l}$, $\sigma_{j, l}$ are nonnegative. For each $j \in I(1, n)$, we pick a Lyapunov function

$$
\left\{\begin{array}{l}
V_{1 j}\left(t, y_{j}\right)=\left(y_{j}\right)^{q} \\
V_{2 j}\left(t, p_{j}\right)=\left(p_{j}\right)^{q}, q \in \mathbb{Z}_{+},
\end{array}\right.
$$

in (51) for (56). Using the Itô differential formula [24], we have

$$
\left\{\begin{aligned}
d V_{1 j}= & {\left[q\left(y_{j}\right)^{q-1}\left(u_{j}^{i-1}-y_{j}\right)\left(\kappa_{j, j}^{i-1} y_{j}+\sum_{l \neq j}^{n} \kappa_{j, l}^{i-1} y_{l}\right)+\frac{1}{2} q(q-1)\left(y_{j}\right)^{q-2}\left(u_{j}^{i-1}-y_{j}\right)^{2}\left(\left(\delta_{j, j}^{i-1}\right)^{2}+\sum_{l \neq j}^{n}\left(\delta_{j, l}^{i-1}\right)^{2} y_{l}^{2}\right)\right] d t } \\
& +q\left(y_{j}\right)^{q-1}\left(u_{j}^{i-1}-y_{j}\right)\left[\delta_{j, j}^{i-1} d W_{j, j}(t)+\sum_{l \neq j}^{n} \delta_{j, l}^{i-1} y_{l} d W_{j, l}(t)\right], y_{j}\left(T_{i-1}\right)=y_{j}^{i-1}, t \in\left[T_{i-1}, T_{i}\right) \\
V_{1 j}^{i}= & \left(\pi_{j}^{i}\right)^{q} y_{j}\left(T_{i}^{-}, T_{i-1}, y^{i-1}\right)^{q}, \text { if } t=T_{i}, \\
d V_{2 j}= & \left(p_{j}\right)^{q}\left[q\left(\gamma_{j}^{i-1}\left(y_{j}-p_{j}\right)+\beta_{j}^{i-1}+\sum_{l \neq j}^{n} \gamma_{j, l}^{i-1} p_{l}\right)+\frac{1}{2} q(q-1)\left(\left(\sigma_{j, j}^{i-1}\right)^{2}+\sum_{l \neq j}^{n}\left(\sigma_{j, l}^{i-1}\right)^{2} p_{l}^{2}\right)\right] d t \\
& +q\left(p_{j}\right)^{q}\left[\sigma_{j, j}^{i-1} d Z_{j, j}(t)+\sum_{l \neq j}^{n} \sigma_{j, l}^{i-1} p_{l} d Z_{j, l}(t)\right], p_{j}\left(T_{i-1}\right)=p_{j}^{i-1}, t \in\left[T_{i-1}, T_{i}\right), \\
V_{2 j}^{i}= & \left(\theta_{j}^{i}\right)^{q} p_{j}\left(T_{i}^{-}, T_{i-1}, y^{i-1}, \mathbf{p}^{i-1}\right)^{q}, \text { if } t=T_{i},
\end{aligned}\right.
$$


By setting $\Delta t_{k}^{i-1}=t_{k}^{i-1}-t_{k-1}^{i-1}=\Delta t, \quad \Delta y\left(t_{k}^{i-1}\right)=\boldsymbol{y}\left(t_{k}^{i-1}\right)-$ $\boldsymbol{y}\left(t_{k-1}^{i-1}\right)$, and $\Delta \mathbf{p}\left(t_{k}^{i-1}\right)=\mathbf{p}\left(t_{k}^{i-1}\right)-\mathbf{p}\left(t_{k-1}^{i-1}\right)$, the combined Euler discretized scheme for (61) is

$$
\left\{\begin{aligned}
& \Delta\left(y_{j}\right)^{q}\left(t_{k}^{i-1}\right)= {\left[q\left(y_{j}\right)^{q-1}\left(t_{k-1}^{i-1}\right)\left(u_{j}^{i-1}-y_{j}\left(t_{k-1}^{i-1}\right)\right)\left(k_{j, j}^{i-1} y_{j}\left(t_{k-1}^{i-1}\right)+\sum_{l \neq j}^{n} k_{j, l}^{i-1} y_{l}\left(t_{k-1}^{i-1}\right)\right)+\frac{1}{2} q(q-1)\left(y_{j}\right)^{q-2}\left(t_{k-1}^{i-1}\right)\left(u_{j}^{i-1}-y_{j}\left(t_{k-1}^{i-1}\right)\right)^{2}\left(\left(\delta_{j, j}^{i-1}\right)^{2}+\sum_{l \neq j}^{n}\left(\delta_{j, l}^{i-1}\right)^{2} y_{l}^{2}\left(t_{k-1}^{i-1}\right)\right)\right] \Delta t } \\
& \quad q\left(y_{j}\right)^{q-1}\left(t_{k-1}^{i-1}\right)\left(u_{j}^{i-1}-y_{j}\left(t_{k-1}^{i-1}\right)\right)\left[\delta_{j, j}^{i-1} \Delta W_{j, j}\left(t_{k}^{i-1}\right)+\sum_{l \neq j}^{n} \delta_{j, l}^{i-1} y_{l} \Delta W_{j, l}\left(t_{k}^{i-1}\right)\right], y_{j}\left(T_{i-1}\right)=y_{j}^{i-1}, t_{k}^{i-1} \in\left[T_{i-1}, T_{i}\right) \\
&\left(y_{j}^{i}\right)^{q}=\left(\pi_{j}^{i}\right)^{q} y_{j}\left(T_{i}^{-}, T_{i-1}, \mathbf{y}^{i-1}\right)^{q}, \text { if } t=T_{i}, \\
& \Delta\left(p_{j}\right)^{q}\left(t_{k}^{i-1}\right)=\left(p_{j}\right)^{q}\left(t_{k-1}^{i-1}\right)\left[q\left(\gamma_{j, j}^{i-1}\left(y_{j}\left(t_{k-1}^{i-1}\right)-p_{j}\left(t_{k-1}^{i-1}\right)\right)+\beta_{j}^{i-1}+\sum_{l \neq j}^{n} \gamma_{j, l}^{i-1} p_{l}\left(t_{k-1}^{i-1}\right)\right)+\frac{1}{2} q(q-1)\left(\left(\sigma_{j, j}^{i-1}\right)^{2}+\sum_{l \neq j}^{n}\left(\sigma_{j, j}^{i-1}\right)^{2} p_{l}^{2}\left(t_{k-1}^{i-1}\right)\right)\right] \Delta t \\
& \quad+q\left(p_{j}\right)^{q}\left(t_{k-1}^{i-1}\right)\left[\sigma_{j, j}^{i-1} \Delta Z_{j, j}\left(t_{k}^{i-1}\right)+\sum_{l \neq j}^{n} \sigma_{j, l}^{i-1} p_{l}\left(t_{k-1}^{i-1}\right) \Delta Z_{j, l}\left(t_{k}^{i-1}\right)\right], p_{j}\left(T_{i-1}\right)=p_{j}^{i-1} t_{k}^{i-1} \in\left[T_{i-1}, T_{i}\right), q \in I(1, n+1),
\end{aligned}\right.
$$

where $\left\{\mathbf{y}\left(t_{k}^{i-1}\right)\right\}_{k=-r_{i-1}}^{0},\left\{\mathbf{p}\left(t_{k}^{i-1}\right)\right\}_{k=-r_{i-1}}^{0}$ are given finite sequence of $\mathscr{F}_{T_{i-1}}^{i-1}$ measurable random vectors and are independent of $\left\{\Delta W\left(t_{k}^{i-1}\right)\right\}_{k=0}^{N_{i-1}},\left\{\Delta Z\left(t_{k}^{i-1}\right)\right\}_{k=0}^{N_{i-1}}$, respectively. We define $\Delta\left(y_{j}\right)^{q}\left(t_{k}^{i-1}\right)=\left(y_{j}\right)^{q}\left(t_{k}^{i-1}\right)-\left(y_{j}\right)^{q}\left(t_{k-1}^{i-1}\right)$ and $\Delta\left(p_{j}\right)^{\mathrm{q}}$ $\left(t_{k}^{i-1}\right)=\left(p_{j}\right)^{q}\left(t_{k}^{i-1}\right)-\left(p_{j}\right)^{q}\left(t_{k-1}^{i-1}\right)$.

Applying conditional expectation to (62) with respect to $\mathscr{F}_{t_{k-1}}^{i-1} \equiv \mathscr{F}_{k-1}^{i-1}$, we obtain

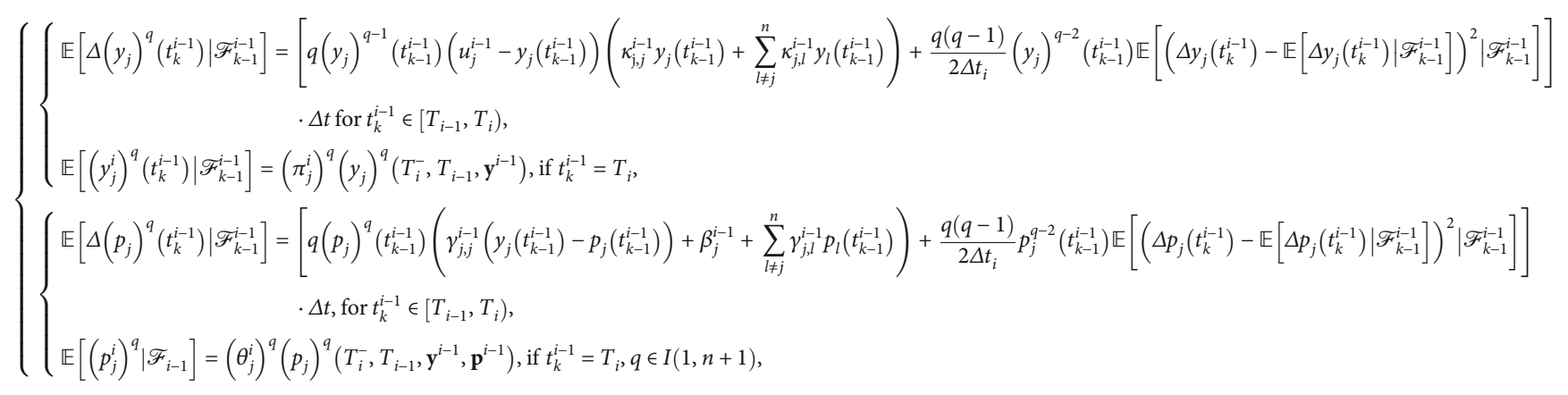

$$
\left\{\begin{array}{l}
\mathbb{E}\left[\left(\Delta\left(y_{j}\right)^{q}\left(t_{k}^{i-1}\right)-\mathbb{E}\left[\Delta\left(y_{j}\right)^{q}\left(t_{k}^{i-1}\right) \mid \mathscr{F}_{k-1}^{i-1}\right]\right)\left(\Delta\left(y_{l}\right)^{q}\left(t_{k}^{i-1}\right)-\mathbb{E}\left[\Delta\left(y_{l}\right)^{q}\left(t_{k}^{i-1}\right) \mid \mathscr{F}_{k-1}^{i-1}\right]\right) \mid \mathscr{F}_{k-1}^{i-1}\right]= \\
q^{2}\left(y_{j} y_{l}\right)^{q-1}\left(t_{k-1}^{i-1}\right)\left(u_{j}^{i-1}-y_{j}\left(t_{k-1}^{i-1}\right)\right)\left(u_{l}^{i-1}-y_{l}\left(t_{k-1}^{i-1}\right)\right)\left[\delta_{j, j}^{i-1} \delta_{l, j}^{i-1} y_{j}\left(t_{k-1}^{i-1}\right)+\delta_{l, l}^{i-1} \delta_{j, l}^{i-1} y_{l}\left(t_{k-1}^{i-1}\right)+\sum_{r=1, j, j \neq r}^{n} \delta_{j, r}^{i-1} \delta_{l, r}^{i-1} y_{r}^{2}\left(t_{k-1}^{i-1}\right)\right] \Delta t \\
t_{k}^{i-1} \in\left[T_{i-1}-\tau_{i-1}, T_{i}\right) \\
\mathbb{E}\left[\left(\Delta\left(p_{j}\right)^{q}\left(t_{k}^{i^{-1}}\right)-\mathbb{E}\left[\Delta\left(p_{j}\right)^{q}\left(t_{k}^{i-1}\right) \mid \mathscr{F}_{k-1}^{i-1}\right]\right)\left(\Delta\left(p_{l}\right)^{q}\left(t_{k}^{i-1}\right)-\mathbb{E}\left[\Delta\left(p_{l}\right)^{q}\left(t_{k}^{i-1}\right) \mid \mathscr{F}_{k-1}^{i-1}\right)\right) \mid \mathscr{F}_{k-1}^{i-1}\right]= \\
q^{2}\left(p_{j} p_{l}\right)^{q}\left(t_{k-1}^{i-1}\right)\left[2 \sigma_{j, j}^{i-1} \sigma_{l, j}^{i-1} y_{j}\left(t_{k-1}^{i-1}\right)+\sum_{r=1, j, l \neq r}^{n} \sigma_{j, r}^{i-1} \sigma_{l, r}^{i-1} y_{r}^{2}\left(t_{k-1}^{i-1}\right)\right], q, j \neq l \in I(1, n),
\end{array}\right.
$$


where $\mathscr{F}_{k-1}^{i-1}$ is the filtration up to time $t_{k-1}^{i-1}$. From (62), (63) and (64) are reduced to

$$
\left\{\begin{array}{l}
\Delta\left(y_{j}\right)^{q}\left(t_{k}^{i-1}\right)=\mathbb{E}\left[\Delta\left(y_{j}\right)^{q}\left(t_{k}^{i-1}\right) \mid \mathscr{F}_{k-1}^{i-1}\right]+q\left(y_{j}\right)^{q-1}\left(t_{k-1}^{i-1}\right)\left(u_{j}^{i-1}-y_{j}\left(t_{k-1}^{i-1}\right)\right)\left[\delta_{j, j}^{i-1} \Delta W_{j, j}\left(t_{k}^{i-1}\right)+\sum_{l \neq j}^{n} \delta_{j, l}^{i-1} y_{l} \Delta W_{j, l}\left(t_{k}^{i-1}\right)\right], \\
y_{j}\left(T_{i-1}\right)=y_{j}^{i-1}, t_{k}^{i-1} \in\left[T_{i-1}, T_{i}\right), \\
\left(y_{j}^{i}\right)^{q}=\left(\pi_{j}^{i}\right)^{q}\left(y_{j}\right)^{q}\left(T_{i}^{-}, T_{i-1}, \mathbf{y}^{i-1}\right), \text { if } t_{k}^{i-1}=T_{i}, \\
\Delta\left(p_{j}\right)^{q}\left(t_{k}^{i-1}\right)=\mathbb{E}\left[\Delta\left(p_{j}\right)^{q}\left(t_{k}^{i-1}\right) \mid \mathscr{F}_{k-1}^{i-1}\right]+q\left(p_{j}\right)^{q}\left(t_{k-1}^{i-1}\right)\left[\sigma_{j, j}^{i-1} \Delta Z_{j, j}\left(t_{k}^{i-1}\right)+\sum_{l \neq j}^{n} \sigma_{j, l}^{i-1} p_{l}\left(t_{k-1}^{i-1}\right) \Delta Z_{j, l}\left(t_{k}^{i-1}\right)\right], \\
p_{j}\left(T_{i-1}\right)=p_{j}^{i-1}, t_{k}^{i-1} \in\left[T_{i-1}, T_{i}\right), q \in I(1, n+1), j \in I(1, n), \\
\left(p_{j}^{i}\right)^{q}=\left(\theta_{j}^{i}\right)^{q}\left(p_{j}\right)^{q}\left(T_{i}^{-}, T_{i-1}, \mathbf{y}^{i-1}, \mathbf{p}^{i-1}\right), \text { if } t_{k}^{i-1}=T_{i} .
\end{array}\right.
$$

Equation (65) provides the basis for the development of the concept of lagged adaptive expectation process [22] with respect to continuous time stochastic dynamic systems (56) and (61).

For $k \in I\left(0, N_{i-1}\right)$, applying the lagged adaptive expectation process [22] and Definitions 6, 8, and 10 and using (63),
(64), and (65), we formulate a local observation/measurement process at $t_{k}^{i-1}$ as an algebraic functions of $m_{k}^{i-1}$ local functions of restriction of the finite sample sequence $\left\{\mathbf{y}\left(t_{l}^{i-1}\right)\right\}_{l=-r_{i-1}}^{N_{i-1}}$ and $\left\{\mathbf{p}\left(t_{l}^{i-1}\right)\right\}_{l=-r_{i-1}}^{N_{i-1}}$ to subpartition $P_{k}^{i-1}$ in Definition 8:

$$
\begin{aligned}
& \frac{1}{m_{k}^{i-1}} \sum_{l=k-m_{k}^{i-1}}^{k-1} \mathbb{E}\left[\Delta\left(y_{j}\right)^{q}\left(t_{l}^{i-1}\right) \mid \mathscr{F}_{l-1}^{i-1}\right]=\left\{\frac { 1 } { m _ { k } ^ { i - 1 } } \sum _ { l = k - m _ { k } ^ { i - 1 } } ^ { k - 1 } \left[q\left(y_{j}\right)^{q-1}\left(t_{l-1}^{i-1}\right)\left(u_{j}^{i-1}-y_{j}\left(t_{t-1}^{i-1}\right)\right)\left(\kappa_{j, j}^{i-1} y_{j}\left(t_{l-1}^{i-1}\right)+\sum_{l \neq j}^{n} \kappa_{j, l}^{i-1} y_{l}\left(t_{l-1}^{i-1}\right)\right)\right.\right. \\
& \left.+\frac{q(q-1)}{2 \Delta t}\left(y_{j}\right)^{q-2}\left(t_{t-1}^{i-1}\right) s_{m_{k}^{i-1}, k, \Delta y_{j}}^{j, j}\right] \Delta t, \\
& \frac{1}{m_{k}^{i-1}} \sum_{l=k-m_{k}^{i-1}}^{k-1} \mathbb{E}\left[\Delta\left(p_{j}\right)^{q}\left(t_{l}^{i-1}\right) \mid \mathscr{F}_{l-1}^{i-1}\right]=\left\{\frac { 1 } { m _ { k } ^ { i - 1 } } \sum _ { l = k - m _ { k } ^ { i - 1 } } ^ { k - 1 } \left[q\left(p_{j}\right)^{q}\left(t_{l-1}^{i-1}\right)\left(\gamma_{j, j}^{i-1}\left(y_{j}\left(t_{l-1}^{i-1}\right)-p_{j}\left(t_{l-1}^{i-1}\right)\right)+\beta_{j}^{i-1}+\sum_{l \neq j}^{n} \gamma_{j, l} p_{l}\left(t_{l-1}^{i-1}\right)\right)\right.\right. \\
& \left.+\frac{q(q-1)}{2 \Delta t} p_{j}^{q-2}\left(t_{t-1}^{i-1}\right) s_{m_{k}^{i-1}, k, \Delta p_{j}}^{j, j}\right] \Delta t, q \in I(1, n+1), \\
& \int \hat{s}_{m_{k}^{i-1}, k}\left(\Delta(y)^{q}\right)=\frac{1}{m_{k}^{i-1}} \sum_{l=k-m_{k}^{i-1}}^{k-1} q^{2}\left(y_{j} y_{l}\right)^{q-1}\left(t_{t-1}^{i-1}\right)\left(u_{j}^{i-1}-y_{j}\left(t_{t-1}^{i-1}\right)\right)\left(u_{l}^{i-1}-y_{l}\left(t_{t-1}^{i-1}\right)\right) \\
& {\left[\delta_{j, j}^{i-1} \delta_{l, j}^{i-1} y_{j}\left(t_{l-1}^{i-1}\right)+\delta_{l, l}^{i-1} \delta_{j, l}^{i-1} y_{l}\left(t_{k-1}^{i-1}\right)+\sum_{r=1 j \neq l \neq r}^{n} \delta_{j, r}^{i-1} \delta_{l, r}^{i-1} y_{r}^{2}\left(t_{l-1}^{i-1}\right)\right]} \\
& \hat{s}_{m_{k}^{i-1}, k}^{i}\left(\Delta(p)^{q}\right)=\frac{1}{m_{k}^{i-1}} \sum_{l=k-m_{k}^{i-1}}^{k-1} q^{2}\left(p_{j} p_{l}\right)^{q}\left(t_{l-1}^{i-1}\right)\left[\sigma_{j, j}^{i-1} \sigma_{l, j}^{i-1} p_{j}\left(t_{l-1}^{i-1}\right)+\sigma_{l, l}^{i-1} \sigma_{j, l}^{i-1} p_{l}\left(t_{l-1}^{i-1}\right)+\sum_{r=1 j \neq l \neq r}^{n} \sigma_{j, r}^{i-1} \sigma_{l, r}^{i-1} p_{r}^{2}\left(t_{l-1}^{i-1}\right)\right], \\
& j \neq l \in I(1, n), q \in I(1,2 n) .
\end{aligned}
$$


For each $i \in I\left(1, K^{*}\right)$ and each $j \neq l \in I(1, n)$, we define

$$
\begin{aligned}
& F_{1 q}\left(u_{j}^{i-1},\left\{\kappa_{j, r}^{i-1}\right\}_{r=1}^{n}\right) \equiv F_{1 q}\left(\mathbb{E}\left[\Delta\left(y_{j}^{q}\right)\left(t_{l}^{i-1}\right) \mid \mathscr{F}_{l-1}^{i-1}\right] ; u_{j}^{i-1},\left\{\kappa_{j, r}^{i-1}\right\}_{r=1}^{n}\right), \\
& F_{2 q}\left(\left\{\delta_{j, r}^{i-1}, \delta_{l, r}^{i-1}\right\}_{r=1}^{n}\right) \equiv F_{2 q}\left(\mathbb{E}\left[\Delta\left(y_{j}^{q}\right)\left(t_{l}^{i-1}\right) \mid \mathscr{F}_{l-1}^{i-1}\right], \mathbb{E}\left[\Delta\left(y_{l}^{q}\right)\left(t_{l}^{i-1}\right) \mid \mathscr{F}_{l-1}^{i-1}\right] ;\left\{\delta_{j, r}^{i-1}, \delta_{l, r}^{i-1}\right\}_{r=1}^{n}\right), \\
& G_{1 q}\left(\beta_{j}^{i-1},\left\{\gamma_{j, r}^{i-1}\right\}_{r=1}^{n}\right) \equiv G_{1 q}\left(\mathbb{E}\left[\Delta\left(p_{j}^{q}\right)\left(t_{l}^{i-1}\right) \mid \mathscr{F}_{l-1}^{i-1}\right] ; \beta_{j}^{i-1},\left\{\gamma_{j, r}^{i-1}\right\}_{r=1}^{n}\right), \\
& G_{2 q}\left(\left\{\sigma_{j, r}^{i-1}, \sigma_{l, r}^{i-1}\right\}_{r=1}^{n}\right) \equiv G_{2 q}\left(\mathbb{E}\left[\Delta\left(p_{j}^{q}\right)\left(t_{l}^{i-1}\right) \mid \mathscr{F}_{l-1}^{i-1}\right], \mathbb{E}\left[\Delta\left(p_{l}^{q}\right)\left(t_{l}^{i-1}\right) \mid \mathscr{F}_{l-1}^{i-1}\right] ;\left\{\sigma_{j, r} \sigma_{l, r}^{i-1}\right\}_{r=1}^{n}\right),
\end{aligned}
$$

by

$$
\begin{aligned}
& F_{1 q}\left(u_{j}^{i-1},\left\{\kappa_{j, r}^{i-1}\right\}_{r=1}^{n}\right)=\frac{1}{m_{k}^{i-1}} \sum_{l=k-m_{k}^{i-1}}^{k-1}\left\{\left[q\left(y_{j}\right)^{q-1}\left(t_{t-1}^{i-1}\right)\left(u_{j}^{i-1}-y_{j}\left(t_{t-1}^{i-1}\right)\right)\left(\sum_{r=1}^{n} \kappa_{j, r} y_{r}\left(t_{t-1}^{i-1}\right)\right)\right.\right. \\
& \left.\left.+\frac{q(q-1)}{2 \Delta t}\left(y_{j}\right)^{q-2}\left(t_{t-1}^{i-1}\right) \operatorname{s}_{m_{k}^{i-1}, k}^{j, j}\left(\Delta y_{j}\right)\right] \Delta t\right\}-\frac{1}{m_{k}^{i-1}} \sum_{l=k-m_{k}^{i-1}}^{k-1} \mathbb{E}\left[\Delta\left(y_{j}\right)^{q}\left(t_{l}^{i-1}\right) \mid \mathscr{F}_{l-1}^{i-1}\right], q \in I(1, n+1), \\
& F_{2 q}\left(\left\{\delta_{j, r}^{i-1}, \delta_{l, r}^{i-1}\right\}_{r=1}^{n}\right)=\frac{1}{m_{k}^{i-1}} \sum_{l=k-m_{k}^{i-1}}^{k-1} q^{2}\left(y_{j} y_{l}\right)^{q-1}\left(t_{l-1}^{i-1}\right)\left(u_{j}^{i-1}-y_{j}\left(t_{l-1}^{i-1}\right)\right) \times\left(u_{l}^{i-1}-y_{l}\left(t_{l-1}^{i-1}\right)\right)\left[\delta_{j, j}^{i-1} \delta_{l, j}^{i-1} y_{j}\left(t_{l-1}^{i-1}\right)+\delta_{l, l}^{i-1} \delta_{j, l}^{i-1} y_{l}\left(t_{l-1}^{i-1}\right)\right. \\
& \left.+\sum_{r=1 j \neq l \neq r}^{n} \delta_{j, r}^{i-1} \delta_{l, r}^{i-1} y_{r}^{2}\left(t_{l-1}^{i-1}\right)\right]-\widehat{s}_{m_{k}^{i-1}, k,}^{j, l}\left(\Delta(y)^{q}\right), j \neq l \in I(1, n), q \in I(1,2 n), \\
& G_{1 q}\left(\beta_{j}^{i-1},\left\{\gamma_{j, r}^{i-1}\right\}_{r=1}^{n}\right)=\frac{1}{m_{k}^{i-1}} \sum_{l=k-m_{k}^{i-1}}^{k-1}\left\{\left[q\left(p_{j}\right)^{q}\left(t_{t-1}^{i-1}\right)\left(\gamma_{j, j}^{i-1}\left(y_{j}\left(t_{l-1}^{i-1}\right)-p_{j}\left(t_{l-1}^{i-1}\right)\right)+\beta_{j}^{i-1}+\sum_{r \neq j}^{n} \gamma_{j, r}^{i-1} p_{r}\left(t_{t-1}^{i-1}\right)\right)\right.\right. \\
& \left.\left.+\frac{q(q-1)}{2 \Delta t} p_{j}^{q-2}\left(t_{t-1}^{i-1}\right) \hat{s}_{m_{k}^{i-1}, k}^{j, j}\left(\Delta p_{j}\right)\right] \Delta t\right\}-\frac{1}{m_{k}^{i-1}} \sum_{l=k-m_{k}^{i-1}}^{k-1} \mathbb{E}\left[\Delta\left(p_{j}\right)^{q}\left(t_{t}^{i-1}\right) \mid \mathscr{F}_{l-1}^{i-1}\right], q \in I(1, n+1), \\
& G_{2 q}\left(\left\{\sigma_{j, r}^{i-1}, \sigma_{l, r}^{i-1}\right\}_{r=1}^{n}\right)=\frac{1}{m_{\mathrm{k}}^{i-1}} \sum_{l=k-m_{k}^{i-1}}^{k-1} q^{2}\left(p_{j} p_{l}\right)^{q}\left(t_{l-1}^{i-1}\right)\left[\sigma_{j, j}^{i-1} \sigma_{l, j}^{i-1} p_{j}\left(t_{l-1}^{i-1}\right)\right]+\sigma_{l, l}^{i-1} \sigma_{j, l}^{i-1} p_{l}\left(t_{l-1}^{i-1}\right) \\
& \left.+\sum_{r=1 j \neq l \neq r}^{n} \sigma_{j, r}^{i-1} \sigma_{l, r}^{i-1} p_{r}^{2}\left(t_{l-1}^{i-1}\right)\right]-\widehat{s}_{m_{k}^{i-1}, k, \Delta(p)^{q}}^{j, l}, j \neq l \in I(1, n), q \in I(1,2 n) .
\end{aligned}
$$


TABle 7: Estimates $\pi_{1}^{i}, \pi_{2}^{i}, \pi_{3}^{i}, \theta_{1}^{i}, \theta_{2}^{i}$, and $\theta_{3}^{i}$.

\begin{tabular}{|c|c|c|c|c|c|c|c|c|}
\hline$i$ & $\begin{array}{l}T_{i} \\
T_{i}\end{array}$ & $\begin{array}{c}\text { Natural gas } \\
\Pi_{1}^{i} \\
\end{array}$ & $\begin{array}{c}\text { Crude oil } \\
\Pi_{2}^{i}\end{array}$ & $\begin{array}{c}\text { Coal } \\
\Pi_{3}^{i} \\
\end{array}$ & $\begin{array}{l}T_{i} \\
T_{i}\end{array}$ & $\begin{array}{c}\text { Natural gas } \\
\theta_{1}^{i} \\
\end{array}$ & $\begin{array}{c}\text { Crude oil } \\
\theta_{2}^{i}\end{array}$ & $\begin{array}{c}\text { Coal } \\
\theta_{3}^{i}\end{array}$ \\
\hline 1 & 17 & 1.0031 & 1.1219 & 1.0256 & 17 & 1.0049 & 1.1219 & 1.0493 \\
\hline 2 & 44 & 0.9213 & 0.9727 & 1.0410 & 44 & 0.9352 & 1.0084 & 0.9249 \\
\hline 3 & 61 & 0.9482 & 0.9671 & 0.9661 & 61 & 0.9997 & 0.9427 & 0.9404 \\
\hline 4 & 87 & 0.8859 & 0.9974 & 0.9653 & 87 & 0.7389 & 1.0452 & 0.9905 \\
\hline 5 & 157 & 1.0435 & 0.9350 & 1.0432 & 157 & 1.0933 & 1.0019 & 1.0049 \\
\hline 6 & 200 & 1.0309 & 1.0199 & 1.0382 & 200 & 0.9826 & 1.0210 & 0.9794 \\
\hline 7 & 422 & 1.0270 & 0.9775 & 0.9669 & 422 & 0.9706 & 0.9939 & 0.9917 \\
\hline 8 & 464 & 0.9581 & 1.0462 & 1.0523 & 464 & 1.0128 & 1.0508 & 1.0324 \\
\hline 9 & 483 & 0.9765 & 0.9787 & 1.0291 & 483 & 1.0382 & 1.0328 & 1.0246 \\
\hline 10 & 502 & 1.0532 & 1.0737 & 1.0136 & 502 & 1.0359 & 1.0073 & 1.0162 \\
\hline 11 & 722 & 0.9812 & 0.9959 & 0.9919 & 722 & 0.9700 & 0.9695 & 1.0011 \\
\hline 12 & 754 & 1.0003 & 1.0009 & 0.9189 & 754 & 1.0137 & 0.9987 & 1.3481 \\
\hline 13 & 870 & 1.0579 & 0.9921 & 1.1378 & 870 & 1.0328 & 1.0033 & 1.1420 \\
\hline 14 & 930 & 1.0275 & 0.9907 & 0.9978 & 930 & 0.9995 & 0.9812 & 1.1848 \\
\hline 15 & 1113 & 1.0009 & 0.9960 & 1.0706 & 1113 & 0.9304 & 0.9801 & 0.9897 \\
\hline
\end{tabular}

For every $j \in I(1, n)$, we have

$$
\left\{\begin{array}{l}
F_{1 q}\left(u_{j}^{i-1},\left\{\kappa_{j, r}^{i-1}\right\}_{r=1}^{n}\right)=0, q \in I(1, n+1), \\
F_{2 q}\left(\left\{\delta_{j, r}^{i-1}, \delta_{l, r}^{i-1}\right\}_{r=1}^{n}\right)=0, q \in I(1,2 n), \\
G_{1 q}\left(\beta_{j}^{i-1},\left\{\gamma_{j, r}^{i-1}\right\}_{r=1}^{n}\right)=0, q \in I(1, n+1), \\
G_{2 q}\left(\left\{\delta_{j, r}^{i-1}, \delta_{l, r}^{i-1}\right\}_{r=1}^{n}\right)=0, q \in I(1,2 n) .
\end{array}\right.
$$

We define $F_{1}=\left\{F_{1 q}\right\}_{q \in I(1, n+1)}, F_{2}=\left\{F_{2 q}\right\}_{q \in I(1, n)}, G_{1}=$ $\left\{G_{1 q}\right\}_{q \in I(1, n+1)}$, and $G_{2}=\left\{G_{2 q}\right\}_{q \in I(1, n)}$. Thus, provided that the determinant of each of the Jacobian matrices $J F_{1}\left(u_{j}^{i-1}\right.$, $\left.\left\{\kappa_{j, r}^{\mathrm{i}-1}\right\}_{r=1}^{n}\right), J F_{2}\left(u_{j}^{i-1},\left\{\delta_{j, r}^{i-1}\right\}_{r=1}^{n}\right), J G_{1}\left(\beta_{j}^{i-1},\left\{\gamma_{j, r}^{i-1}\right\}_{r=1}^{n}\right)$ and $J$ $G_{2}\left(\left\{\sigma_{j, r}^{i-1}, \sigma_{l, r}^{i-1}\right\}_{r=1}^{n}\right)$ are not zero, by the application of
Theorem 11 (Implicit Function Theorem), we conclude that for every nonconstant $m_{k}^{i-1}$-local sequence $\left\{y_{j}\left(t_{l}^{i-1}\right)\right\}_{l=k-m_{k}^{i-1}}^{k-1}$ and $\left\{p_{j}\left(t_{l}^{i-1}\right)\right\}_{l=k-m_{k}^{i-1}}^{k-1}$, there exist a unique solution $\left(\widehat{u}_{j}^{i-1}\left(m_{k}^{i-1}, t_{k}^{i-1}\right), \widehat{\beta}_{j}^{i-1}\left(m_{k}^{i-1}, t_{k}^{i-1}\right), \quad\left\{\widehat{\kappa}_{j, r}^{i-1}\right\}_{r=1}^{n}\left(m_{k}^{i-1}\right.\right.$, $\left.t_{k}^{i-1}\right), \quad\left\{\widehat{\gamma}_{j, r}^{i-1}\right\}_{r=1}^{n}\left(m_{k}^{i-1}, t_{k}^{i-1}\right), \quad\left\{\widehat{\delta}_{j, r}^{i-1}\right\}_{r=1}^{n}\left(m_{k}^{i-1}, t_{k}^{i-1}\right), \quad\left\{\widehat{\sigma}_{j, r}^{i-1}\right\}_{r=1}^{n}$ $\left.\left(m_{k}^{i-1}, t_{k}^{i-1}\right)\right)$ of system of algebraic equations (70) as a point estimates of $u_{j}^{i-1}, \kappa_{j, r}^{i-1_{r=1}^{n}}, \gamma_{j, r}^{i-1_{r=1}^{n}}, \delta_{j, r}^{i-1_{r=1}^{n}}, \sigma_{j, r}^{i-1_{r=1}^{n}}, j$ $\in I(1, n)$, respectively. We illustrate this approach using energy commodities natural gas, crude oil and coal [35-37] in the next section.

6.2. Illustration: Application to Energy Commodity. In this subsection, we present an illustration of the derived model on the natural gas, crude oil and coal [35-37]. Here, $j \in I(1,3)$ and $i \in I\left(1, K^{*}\right)$. Moreover, (56) reduces to

$$
\left\{\begin{array}{l}
d y_{j}=\left(u_{j}^{i-1}-y_{j}\right)\left[\kappa_{j, j}^{i-1} y_{j}+\sum_{l \neq j}^{3} \kappa_{j, l}^{i-1} y_{l}\right] d t+\delta_{j, j}^{i-1}\left(u_{j}^{i-1}-y_{j}\right) \mathrm{d} W_{j, j}(t)+\left(u_{j}^{i-1}-y_{j}\right) \sum_{l \neq j}^{3} \delta_{j, l}^{i-1} y_{l} d W_{j, l}(t), y_{j}\left(T_{i-1}\right)=y_{j}^{i-1}, t \in\left[T_{i-1}, T_{i}\right), \\
y_{j}^{i}=\pi_{j}^{i} y_{j}\left(T_{i}^{-}, T_{i-1}, \mathrm{y}^{i-1}\right), \\
d p_{j}(t)=p_{j}\left[\gamma_{j, j}^{i-1}\left(y_{j}-p_{j}\right)+\beta_{j}^{i-1}+\sum_{l \neq j}^{3} \gamma_{j, l}^{i-1} p_{l}(t)\right] d t+\sigma_{j, j}^{i-1} p_{j} d Z_{j, j}(t)+p_{j} \sum_{l \neq j}^{3} \sigma_{j, l}^{i-1} p_{l} d Z_{j, l}(t), p_{j}\left(T_{i-1}\right)=p_{j}^{i-1}, t \in\left[T_{i-1}, T_{i}\right), \\
p_{j}^{i}=\theta_{j}^{i} p_{j}\left(T_{i}^{-}, T_{i-1}, \mathbf{y}^{i-1}, \mathbf{p}^{i-1}\right) .
\end{array}\right.
$$




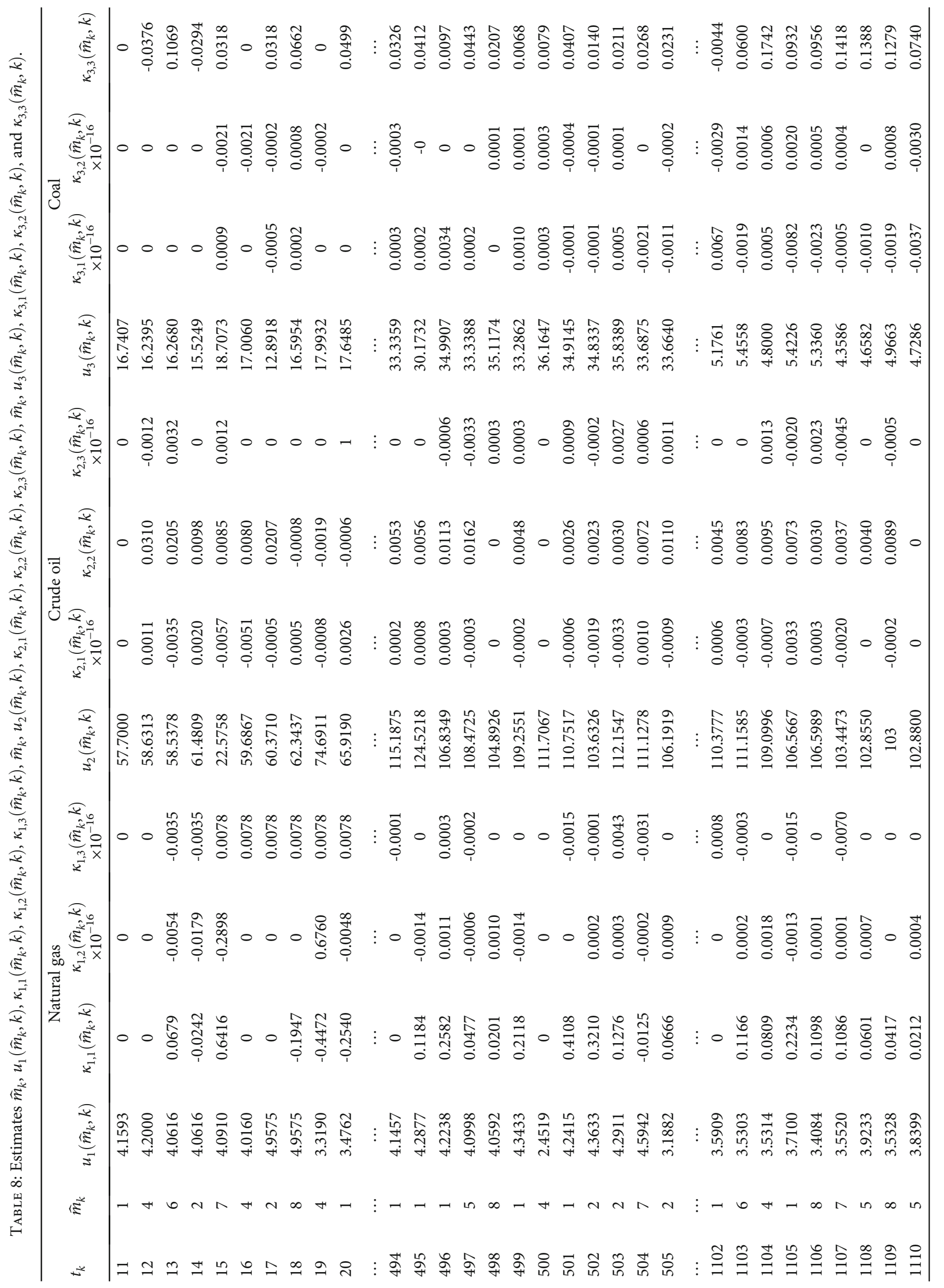


For each $j \in I(1,3)$, following the argument used in Section 6.1, we have

$$
\begin{aligned}
& F_{1 q}\left(u_{j}^{i-1},\left\{\kappa_{j, r}^{i-1}\right\}_{r=1}^{3}\right)= \frac{1}{m_{k}^{i-1}} \sum_{l=k-m_{k}^{i-1}}^{k-1}\left\{\left[q\left(y_{j}\right)^{q-1}\left(t_{l-1}^{i-1}\right)\left(u_{j}^{i-1}-y_{j}\left(t_{l-1}^{i-1}\right)\right)\left(\sum_{r=1}^{3} \kappa_{j, r} y_{r}\left(t_{l-1}^{i-1}\right)\right)+\frac{q(q-1)}{2 \Delta t}\left(y_{j}\right)^{q-2}\left(t_{l-1}^{i-1}\right) \widehat{s}_{m_{k}^{j i}, k}^{i, j}\left(\Delta y_{j}\right)\right] \Delta t\right\} \\
&-\frac{1}{m_{k}^{i-1}} \sum_{l=k-m_{k}^{i-1}}^{k-1} \mathbb{E}\left[\Delta\left(y_{j}\right)^{q}\left(t_{l}^{i-1}\right) \mid \mathscr{F}_{l-1}^{i-1}\right], q \in I(1,4), \\
& F_{2 q}\left(\left\{\delta_{j, r}^{i-1}, \delta_{l, r}^{i-1}\right\}_{r=1}^{3}\right)= \frac{1}{m_{k}^{i-1}} \sum_{l=k-m_{k}^{i-1}}^{k-1} q^{2}\left(y_{j} y_{l}\right)^{q-1}\left(t_{l-1}^{i-1}\right)\left(u_{j}^{i-1}-y_{j}\left(t_{l-1}^{i-1}\right)\right) \times\left(u_{l}^{i-1}-y_{l}\left(t_{l-1}^{i-1}\right)\right)\left[\delta_{j, j}^{i-1} \delta_{l, j}^{i-1} y_{j}\left(t_{t-1}^{i-1}\right)+\delta_{l, l}^{i-1} \delta_{j, l}^{i-1} y_{l}\left(t_{l-1}^{i-1}\right)\right. \\
&\left.+\sum_{r=1}^{3} \delta_{j, r}^{i-1} \delta_{l, r}^{i-1} y_{r}^{2}\left(t_{l-1}^{i-1}\right)\right]-\widehat{s}_{m_{k}^{i-1}, k,}\left(\Delta(y)^{q}\right), j \neq l \in I(1,3), q \in I(1,6), \\
& j \neq l \neq r
\end{aligned}
$$

$$
\begin{aligned}
G_{1 q}\left(\beta_{j}^{i-1},\left\{\gamma_{j, r}^{i-1}\right\}_{r=1}^{3}\right)= & \frac{1}{m_{k}^{i-1}} \sum_{l=k-m_{k}^{i-1}}^{k-1}\left\{\left[q\left(p_{j}\right)^{q}\left(t_{l-1}^{i-1}\right)\left(\gamma_{j, j}^{i-1}\left(y_{j}\left(t_{l-1}^{i-1}\right)-p_{j}\left(t_{l-1}^{i-1}\right)\right)+\beta_{j}^{i-1}+\sum_{r \neq j}^{3} \gamma_{j, r}^{i-1} p_{r}\left(t_{l-1}^{i-1}\right)\right)\right.\right. \\
& \left.\left.+\frac{q(q-1)}{2 \Delta t} p_{j}^{q-2}\left(t_{l-1}^{i-1}\right) \widehat{s}_{m_{k}^{j, j}, k}^{i-1}\left(\Delta p_{j}\right)\right] \Delta t\right\}-\frac{1}{m_{k}^{i-1}} \sum_{l=k-m_{k}^{i-1}}^{k-1} \mathbb{E}\left[\Delta\left(p_{j}\right)^{q}\left(t_{l}^{i-1}\right) \mid \mathscr{F}_{l-1}^{i-1}\right], \quad q \in I(1,4), \\
G_{2 q}\left(\left\{\sigma_{j, r}^{i-1}, \sigma_{l, r}^{i-1}\right\}_{r=1}^{3}\right)= & \frac{1}{m_{k}^{i-1}} \sum_{l=k-m_{k}^{i-1}}^{k-1} q^{2}\left(p_{j} p_{l}\right)^{q}\left(t_{l-1}^{i-1}\right)\left[\sigma_{j, j}^{i-1} \sigma_{l, j}^{i-1} p_{j}\left(t_{l-1}^{i-1}\right)+\sigma_{l, l}^{i-1} \sigma_{j, l}^{i-1} p_{l}\left(t_{l-1}^{i-1}\right)+\sum_{r=1 j \neq l \neq r}^{3} \sigma_{j, r}^{i-1} \sigma_{l, r}^{i-1} p_{r}^{2}\left(t_{l-1}^{i-1}\right)\right] \\
& \left.-\widehat{s}_{m_{k}^{j, l}, \mathrm{k}, \Delta(p)^{q}, j \neq l \in I(1,3), q \in I(1,6),}\right] \\
F_{1 q}\left(u_{j}^{i-1},\left\{\kappa_{j, r}^{i-1}\right\}_{r=1}^{3}\right)= & 0, q \in I(1,4), \\
F_{2 q}\left(\left\{\delta_{j, r}^{i-1}, \delta_{l, r}^{i-1}\right\}_{r=1}^{3}\right)= & 0, q \in I(1,6), \\
G_{1 q}\left(\beta_{j}^{i-1},\left\{\gamma_{j, r}^{i-1}\right\}_{r=1}^{3}\right)= & 0, q \in I(1,4), \\
G_{2 q}\left(\left\{\delta_{j, r}^{i-1}, \delta_{l, r}^{i-1}\right\}_{r=1}^{3}\right)= & 0, q \in I(1,6) .
\end{aligned}
$$

We also have $F_{1}=\left\{F_{1 q}\right\}_{q \in I(1,4)}, F_{2}=\left\{F_{2 q}\right\}_{q \in I(1,3)}, G_{1}=$ $\left\{G_{1 q}\right\}_{q \in I(1,4)}$, and $G_{2}=\left\{G_{2 q}\right\}_{q \in I(1,3)}$. For each $j \in I(1,3)$, it follows that the determinant of the Jacobian of $F_{1}\left(u_{j}^{i-1}\right.$, $\left.\left\{\kappa_{j, r}^{i-1}\right\}_{r \in I(1,3)}\right)$, that is,

$J F_{1}\left(u_{j}^{i-1},\left\{\kappa_{j, r}^{i-1}\right\}_{r \in I(1,3)}\right)$, is not zero provided that all parameters $\left\{\kappa_{j, r}\right\}_{r \in I(1,3)}$ are not zero or provided the sequence $\left\{y_{j}^{i-1}\left(t_{r}^{i-1}\right)\right\}_{r=k-m_{k}^{i-1}-1}^{k-1}$ is neither zero nor constant.
Likewise, determinants of the Jacobians $J F_{2}\left(u_{j}^{i-1}\right.$, $\left.\left\{\delta_{j, r}^{i-1}\right\}_{r \in I(1, n)}\right), J G_{1}\left(\beta_{j}^{i-1},\left\{\gamma_{j, r}^{i-1}\right\}_{r \in I(1, n)}\right)$ and $J G_{2}\left(\left\{\sigma_{j, r}^{i-1}\right\}_{r \in I(1, n)}\right)$ are nonzero if $\left\{\delta_{j, r}\right\}_{j, l \in I(1,3)},\left\{\gamma_{j, r}\right\}_{j, l \in I(1,3)}$, and $\left\{\sigma_{j, r}\right\}_{j, l \in I(1,3)}$ are not zero or provided the sequence $\left\{y_{j}^{i-1}\left(t_{r}^{i-1}\right)\right\}_{r=k-m_{k}^{i-1}-1}^{k-1}$ and $\left\{p_{j}^{i-1}\left(t_{r}^{i-1}\right)\right\}_{r=k-m_{k}^{i-1}-1}^{k-1}$ are neither zero nor constant for $j$ $\in I(1,3)$. 


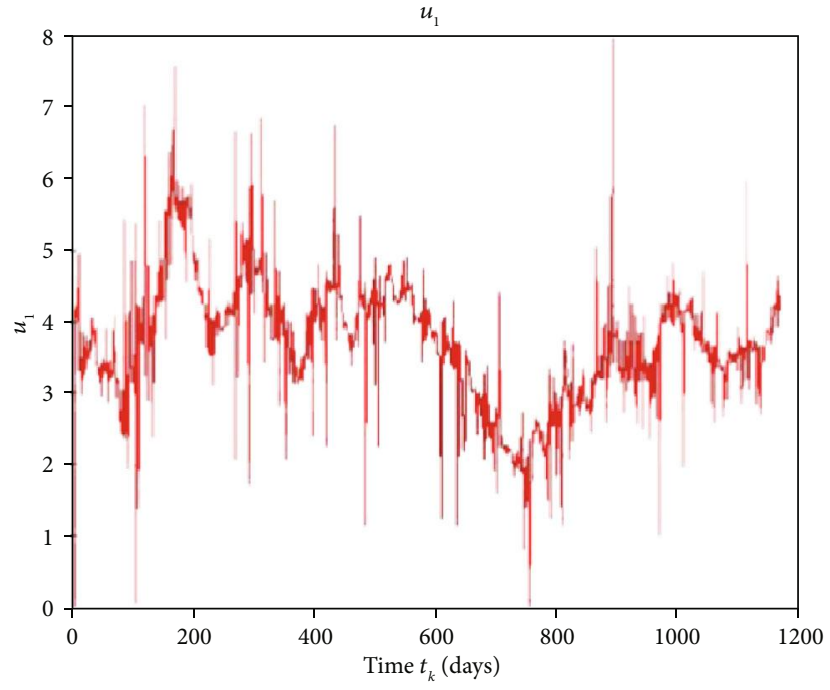

(a)

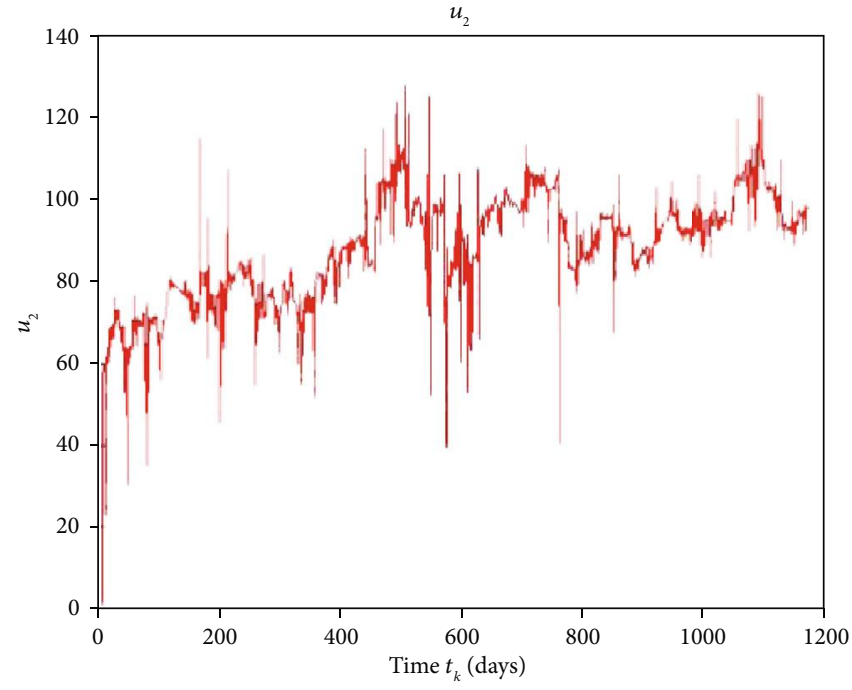

(b)

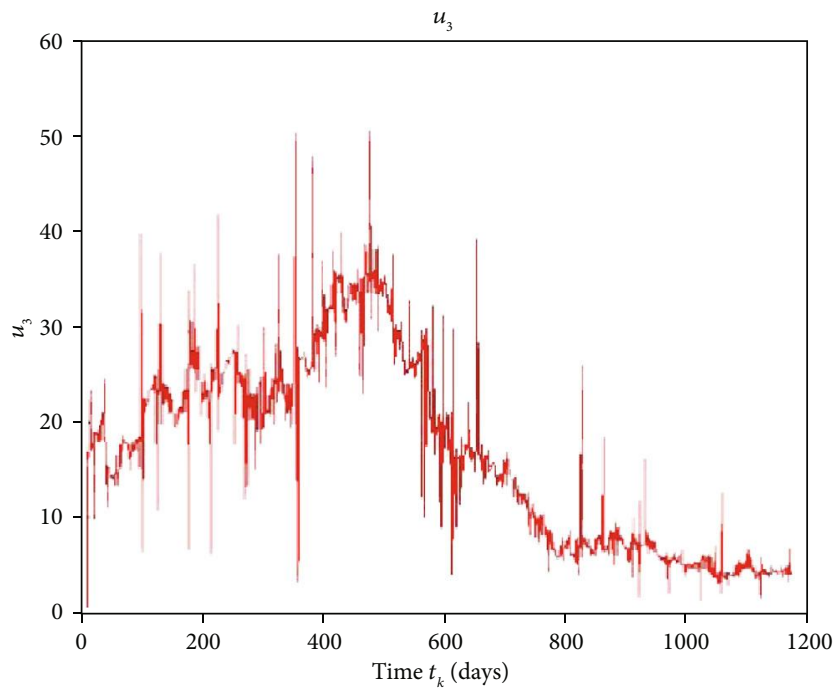

(c)

FIGURE 8: The graph of mean level $u_{1}\left(\widehat{m}_{k}, k\right), u_{2}\left(\widehat{m}_{k}, k\right)$, and $u_{3}\left(\widehat{m}_{k}, k\right)$ for natural gas, crude oil, and coal, respectively.

Remark 13. If the sample $\left\{y_{j}^{i-1}\left(t_{r}^{i-1}\right)\right\}_{r=k-m_{k}^{i-1}-1}^{k-1}$ is a constant sequence, it follows from (62) $(q=1)$ and the fact that $\Delta\left(y_{j}^{i-1}\left(t_{k}^{i-1}\right)\right)=0$ and $s_{m_{k}^{i-1}, k}^{j, j}\left(\Delta y_{j}\right)=0$ that we can set $u_{j}^{i-1}$ $\left(m_{k}^{i-1}, t_{k}^{i-1}\right)=1 / m_{k}^{i-1} \sum_{r=k-m_{k}^{i-1}}^{k-1} y_{j}^{i-1}\left(m_{k}^{i-1}, t_{k}^{i-1}\right)$. It also follows from (66) that $\kappa_{j, r}^{i-1}\left(m_{k}^{i-1}, t_{k}^{i-1}\right)=0$.

\section{Computational Algorithm}

In this section, we outline computational, data organizational, and simulation schemes. We introduce the ideas of iterative data process and data simulation time schedules in relation to the real-time data observation/collection schedule. For the computational estimation of continuous time sto- chastic dynamic system state and parameters, it is essential to identify an admissible set of local conditional sample average and sample covariance parameters, namely, the size of local conditional sample in the context of a partition of time interval $\left[T_{i-1}-\tau_{i-1}, T_{i}\right]$. Moreover, the discrete-time dynamic model of conditional sample mean and sample covariance statistic processes in Section 5 and the theoretical parameter estimation scheme in Section 6 motivates to outline a computational scheme in a systematic and coherent manner. A brief conceptual computational scheme and simulation process summary is described below.

7.1. Coordination of Data Observation, Iterative Process, and Simulation Schedules. Without loss of generality, we assume that the real data observation/collection partition schedules 


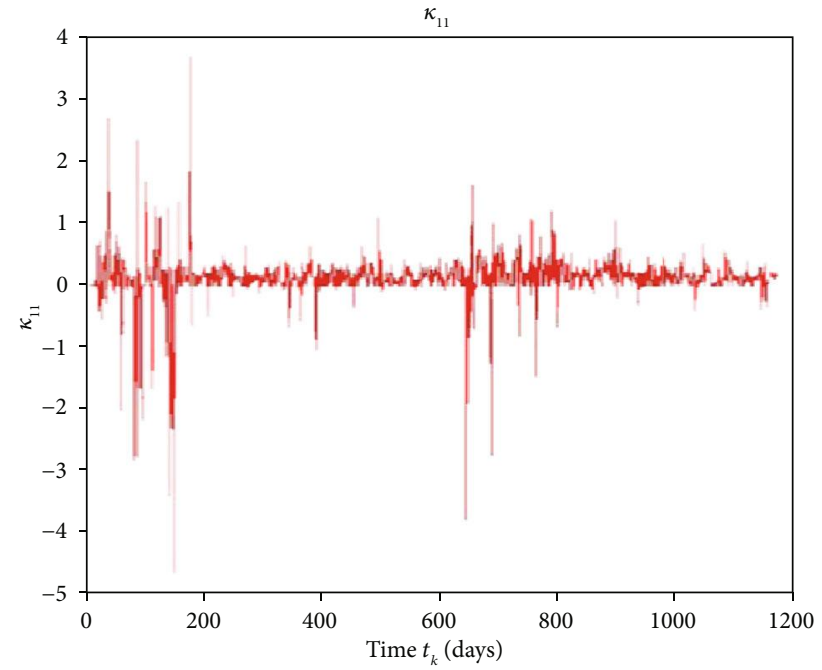

(a)

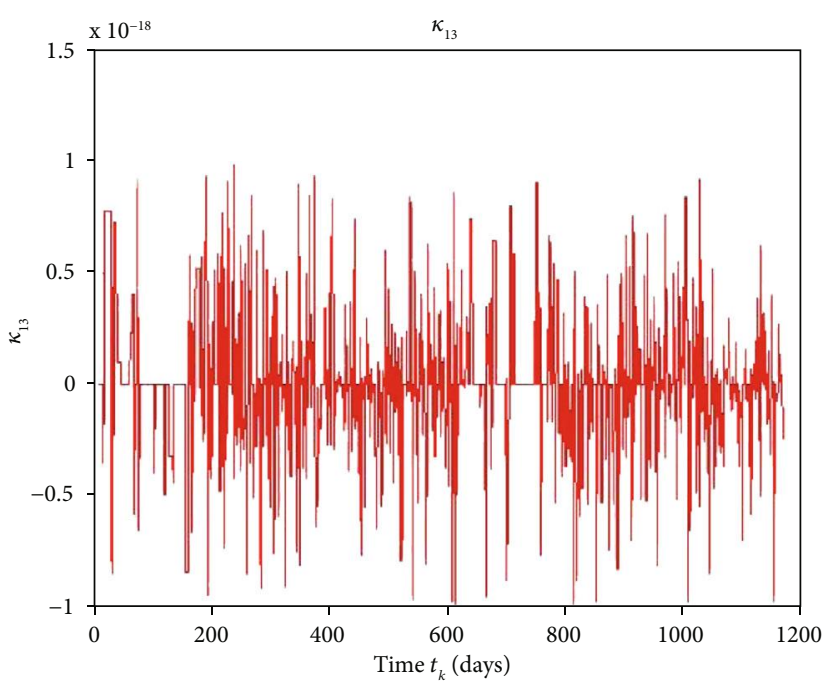

(c)

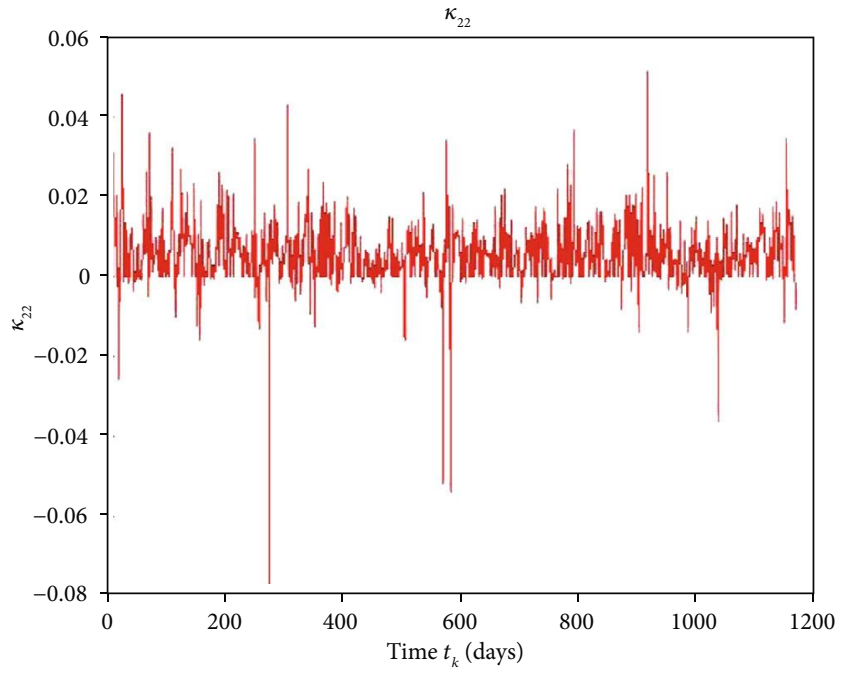

(e)

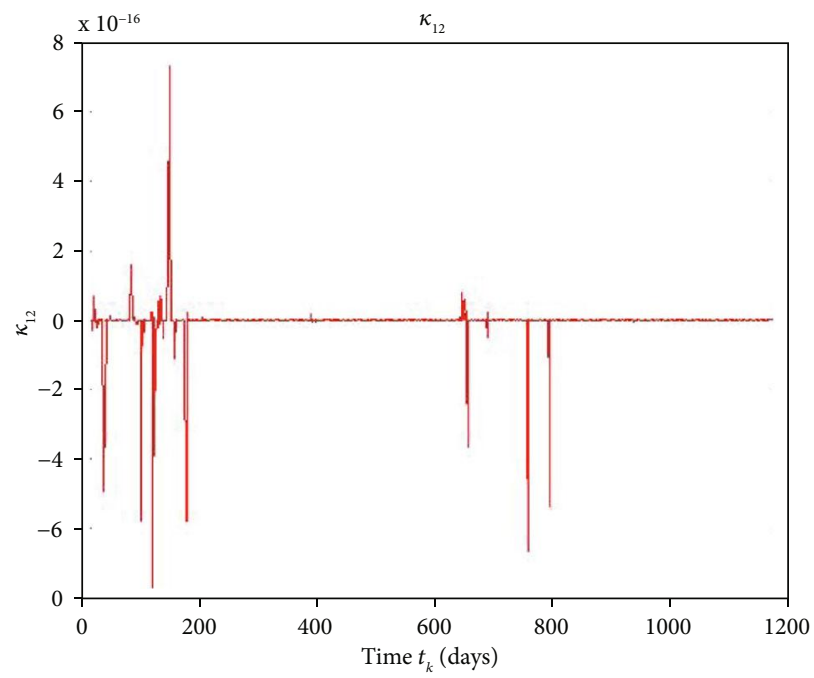

(b)

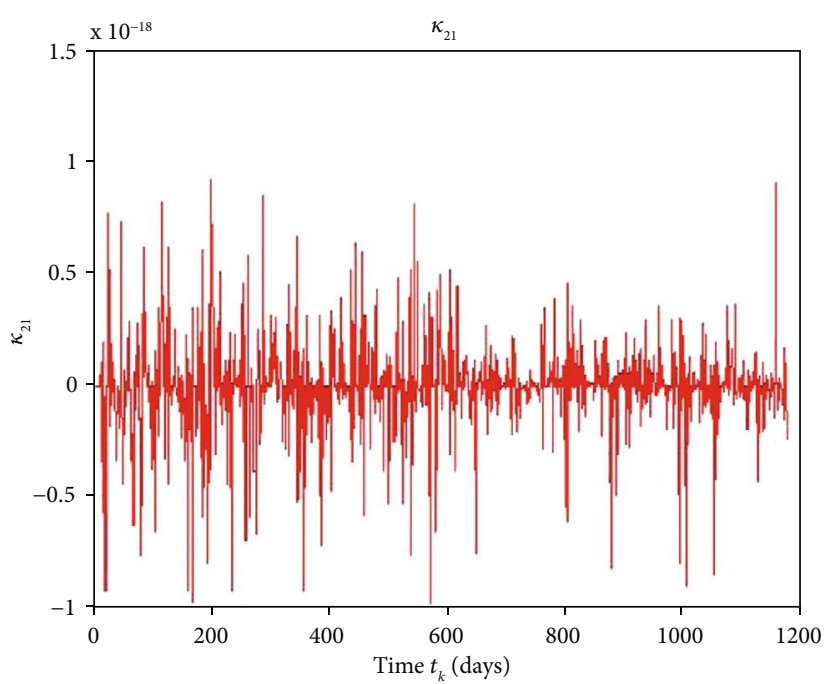

(d)

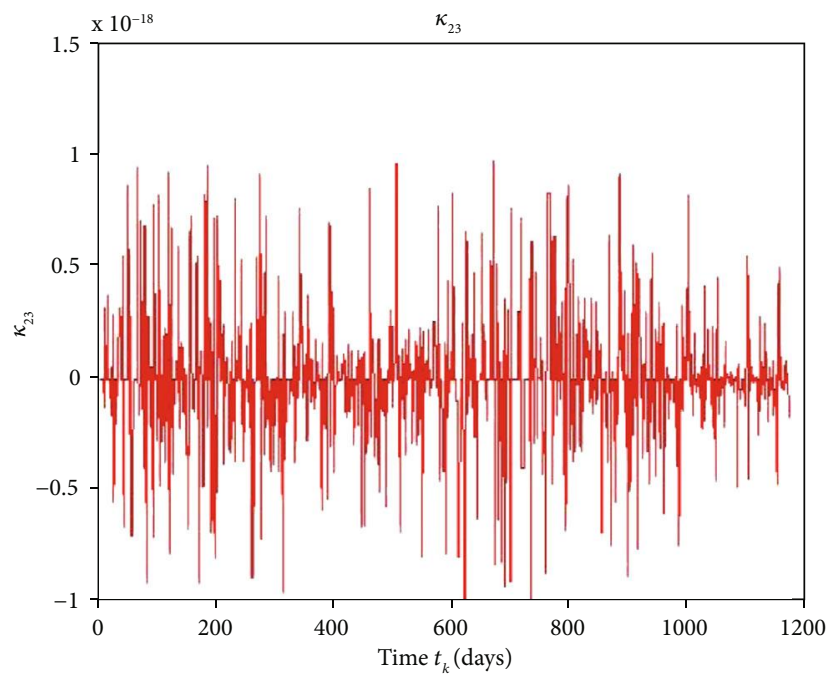

(f)

Figure 9: Continued. 


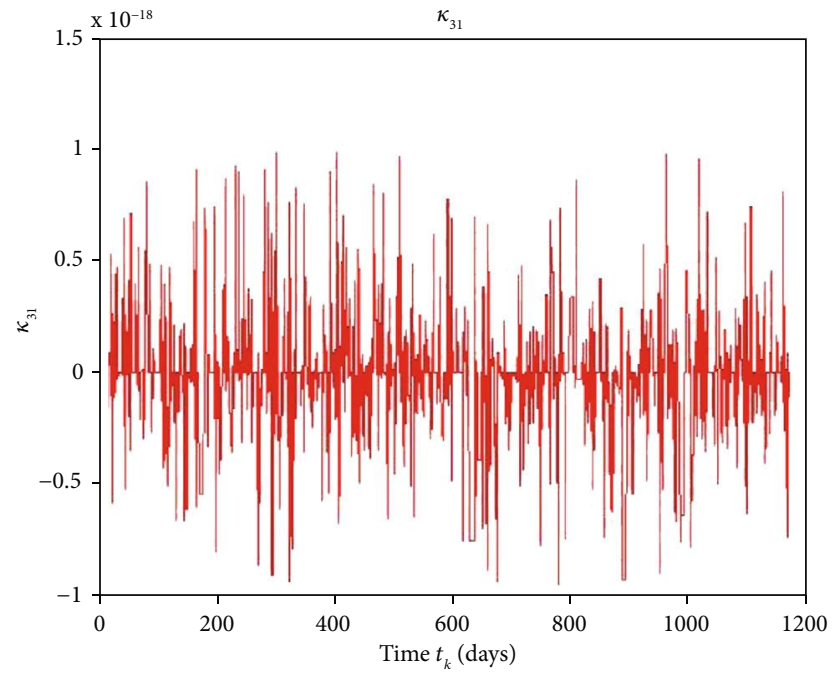

(g)

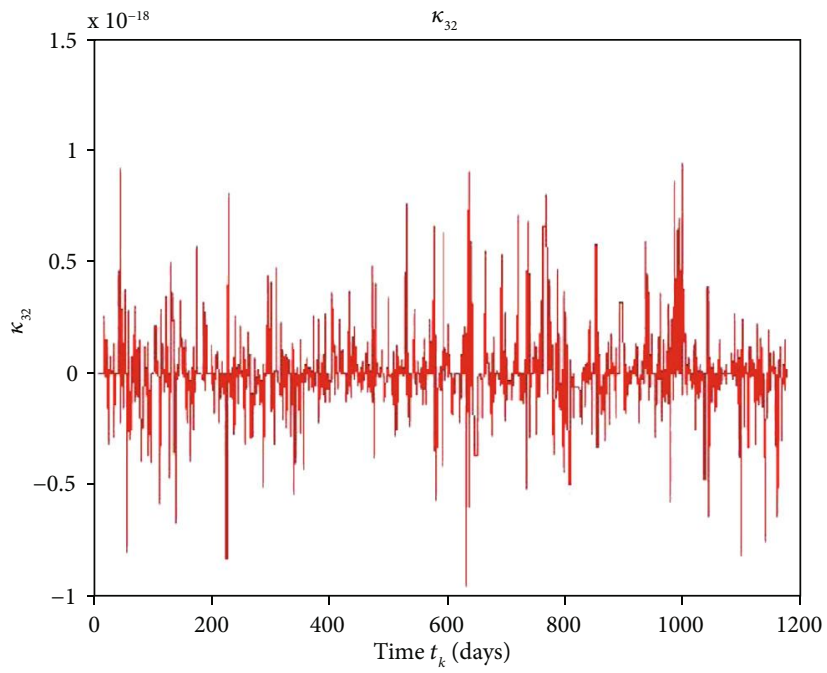

(h)

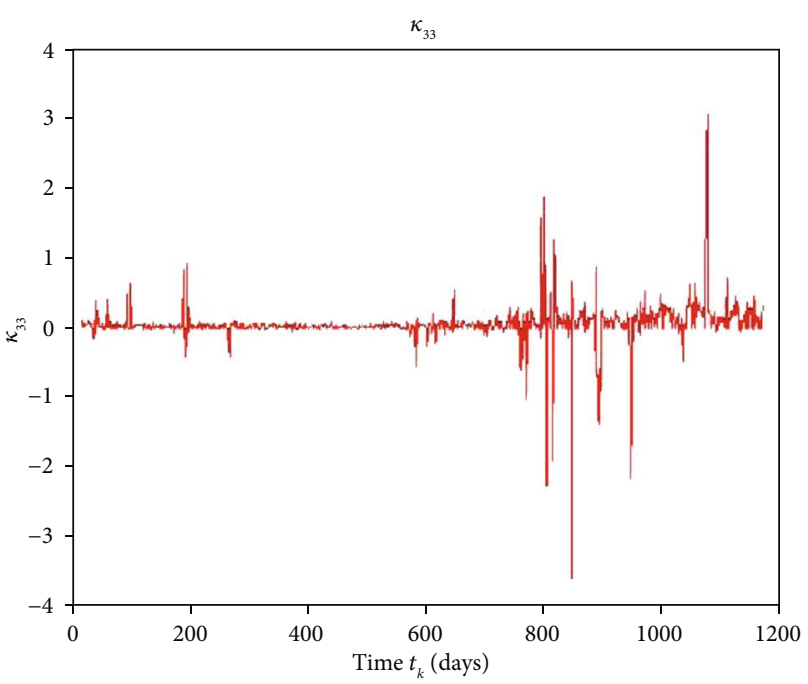

(i)

FIGURE 9: The graph of interaction coefficients $\kappa_{1,1}\left(\widehat{m}_{k}, k\right), \kappa_{1,2}\left(\widehat{m}_{k}, k\right), \kappa_{1,3}\left(\widehat{m}_{k}, k\right), \kappa_{2,1}\left(\widehat{m}_{k}, k\right), \kappa_{2,2}\left(\widehat{m}_{k}, k\right), \kappa_{2,3}\left(\widehat{m}_{k}, k\right), \kappa_{3,1}\left(\widehat{m}_{k}, k\right), \kappa_{3,2}\left(\widehat{m}_{k}, k\right)$, and $\kappa_{3,3}\left(\widehat{m}_{k}, k\right)$.

$\mathbb{P}^{i-1}, i \in I\left(1, K^{*}\right)$ are defined in (41). We present definitions of iterative process and simulation time schedule following the definitions in Otunuga et al. [16].

Definition 14. The iterative process time schedule in relation with the real data collection schedule is defined by

$\left\{I \mathbb{P}^{i-1}=\left\{F^{-r_{i-1}} t_{k}^{i-1}:\right.\right.$ for $\left.t_{k}^{i-1} \in \mathbb{P}^{i-1}\right\}$, for $i \in I\left(1, K^{*}\right), k \in I\left(-r_{i-1}, N_{i-1}\right)$,

where $F^{-r_{i-1}} t_{k}^{i-1}=t_{k-r_{i-1}}^{i-1}$ is a forward shift operator [32].

The simulation time is based on the order $d_{i-1}$ of the time series model of $m_{k}^{i-1}$ local conditional sample mean and covariance processes in (48).
Remark 15. For the case where $K=0$, we have $I \mathbb{P}_{i-1}=I \mathbb{P}$, where $\mathbb{P}^{i-1}=\mathbb{P}$ is defined in (40). This is the iterative time schedule in the absence of jumps.

Definition 16. The simulation process time schedule in relation with the real data observation schedule is defined by

$$
S \mathbb{P}^{i-1}= \begin{cases}\left\{F^{r_{i-1}} t_{k}^{i-1}: \text { for } t_{k}^{i-1} \in \mathbb{P}^{i-1}\right\}, & \text { if } d_{i-1} \leq r_{i-1}, \\ \left\{F^{d_{i-1}} t_{k}^{i-1}: \text { for } t_{k}^{i-1} \in \mathbb{P}^{i-1}\right\}, & \text { if } d_{i-1}>r_{i-1}, k \in I\left(-r_{i-1}, N_{i-1}\right) .\end{cases}
$$

Remark 17. For each $i \in I\left(1, K^{*}\right)$, the initial times of iterative and simulation processes are equal to the real data 
TABLE 9: Estimates $\delta_{1,1}\left(\widehat{m}_{k}, k\right), \delta_{1,2}\left(\widehat{m}_{k}, k\right), \delta_{1,3}\left(\widehat{m}_{k}, k\right), \delta_{2,1}\left(\widehat{m}_{k}, k\right), \delta_{2,2}\left(\widehat{m}_{k}, k\right), \delta_{2,3}\left(\widehat{m}_{k}, k\right), \delta_{3,1}\left(\widehat{m}_{k}, k\right), \delta_{3,2}\left(\widehat{m}_{k}, k\right)$, and $\delta_{3,3}\left(\widehat{m}_{k}, k\right)$.

\begin{tabular}{|c|c|c|c|c|c|c|c|c|c|}
\hline \multirow[b]{2}{*}{$t_{k}$} & \multicolumn{3}{|c|}{ Natural gas } & \multicolumn{3}{|c|}{ Crude oil } & \multicolumn{3}{|c|}{ Coal } \\
\hline & $\delta_{1,1}\left(\widehat{m}_{k}, k\right)$ & $\delta_{1,2}\left(\widehat{m}_{k}, k\right)$ & $\delta_{1,3}\left(\hat{m}_{k}, k\right)$ & $\delta_{2,1}\left(\widehat{m}_{k}, k\right)$ & $\delta_{2,2}\left(\widehat{m}_{k}, k\right)$ & $\delta_{2,3}\left(\widehat{m}_{k}, k\right)$ & $\delta_{3,1}\left(\widehat{m}_{k}, k\right)$ & $\delta_{3,2}\left(\widehat{m}_{k}, k\right)$ & $\delta_{3,3}\left(\widehat{m}_{k}, k\right)$ \\
\hline 11 & 0.0062 & 0.0010 & 0.0001 & 1.5277 & 0.0078 & 0.0011 & 0 & 0 & 0.0218 \\
\hline 12 & 0.0182 & 0.9002 & 0 & 1.6227 & 0.0010 & 0 & 0 & 0 & 0.0988 \\
\hline 13 & 0.0239 & 0.0802 & 1.7280 & 0 & 1.7694 & 0 & 0.6374 & 0.6374 & 0.0959 \\
\hline 14 & 0 & 0.0001 & 0.6027 & 2.3258 & 0 & 0 & 1.6564 & 1.6564 & 0.0847 \\
\hline 15 & 0 & 0.8001 & 0.6210 & 2.3252 & 0 & 0 & 1.6650 & 1.6650 & 0.0111 \\
\hline 16 & 0.0455 & 0.0007 & 3.6877 & 2.3217 & 0 & 1.2215 & 1.6724 & 1.6724 & 0 \\
\hline 17 & 0 & 0.9876 & 0 & 1.6425 & 0 & 0 & 1.7719 & 1.7719 & 0 \\
\hline 18 & 3.0410 & 0.9351 & 0 & 1.3105 & 0 & 0.1070 & 1.7630 & 1.7630 & 0.0434 \\
\hline 19 & 2.7713 & 0.6680 & 0 & 1.1052 & 0 & 0 & 1.7400 & 1.7400 & 0 \\
\hline 20 & 2.8461 & 1.7795 & 0 & 0.1196 & 0 & 0.0983 & 0 & 0.4555 & 0 \\
\hline$\ldots$ & $\ldots$ & $\ldots$ & $\ldots$ & $\ldots$ & $\ldots$ & $\ldots$ & $\ldots$ & $\ldots$ & $\ldots$ \\
\hline 495 & 1.1229 & 0 & 0.0584 & 0.5488 & 0.1104 & 0.0761 & 0 & 0 & 1.3987 \\
\hline 496 & 0.6946 & 0 & 0.6613 & 0.5767 & 0.0715 & 0.0610 & 0 & 0 & 1.3017 \\
\hline 497 & 1.1229 & 0.0095 & 0.0988 & 0.6499 & 0.0870 & 0.0633 & 1.1317 & 1.1317 & 1.3069 \\
\hline 498 & 0.6946 & 0.0101 & 0 & 0 & 0 & 0.0320 & 1.0294 & 1.0294 & 1.5410 \\
\hline 499 & 0.7353 & 0.0066 & 0.0384 & 0 & 0.0922 & 0.0330 & 0.7317 & 0.7317 & 1.2225 \\
\hline 500 & 1.7509 & 0.0069 & 0.0283 & 0.4307 & 0.4545 & 0.0413 & 0.4826 & 0.4826 & 1.2254 \\
\hline 501 & 2.1299 & 0.0077 & 0.0282 & 0.5043 & 0.7873 & 0.0308 & 0.4272 & 0.4272 & 1.5587 \\
\hline 502 & 0.9778 & 0.0077 & 0 & 0.2878 & 0 & 0 & 0.5239 & 0.5239 & 1.8713 \\
\hline 503 & 0.9872 & 0 & 0 & 0.2909 & 0 & 0 & 1.4523 & 1.4523 & 1.8874 \\
\hline 504 & 1.1329 & 0 & 0 & 0.3707 & 0.4261 & 0 & 0 & 0 & 0 \\
\hline 505 & 1.9178 & 0 & 0 & 0.3812 & 0.7292 & 0.1724 & 0 & 0 & 0 \\
\hline$\ldots$ & $\ldots$ & $\ldots$ & $\ldots$ & $\ldots$ & $\ldots$ & $\cdots$ & $\cdots$ & $\cdots$ & $\cdots$ \\
\hline 1102 & 0 & 0.0331 & 0.7183 & 0.9297 & 0.0434 & 0.0680 & 0 & 0 & 1.1355 \\
\hline 1103 & 1.5077 & 0.0626 & 0.2048 & 1.1017 & 0.0421 & 0.1510 & 0 & 0 & 1.4133 \\
\hline 1104 & 0.4444 & 0.0435 & 0.4622 & 0.1939 & 0.1078 & 0 & 0.0814 & 0 & 1.1672 \\
\hline 1105 & 3.5933 & 0 & 0.3646 & 0.1922 & 0 & 0.7273 & 0.2726 & 0.2726 & 1.3023 \\
\hline 1106 & 2.4964 & 0 & 0.3919 & 0 & 0.0684 & 1.0179 & 0.3296 & 0.3296 & 1.4111 \\
\hline 1107 & 2.4600 & 0 & 0.8995 & 0.2001 & 0.1510 & 0.9354 & 0 & 0 & 1.7245 \\
\hline 1108 & 2.0262 & 0 & 0.6325 & 0.3781 & 0.0814 & 0.8825 & 0.1878 & 0.1878 & 1.0915 \\
\hline 1109 & 1.7828 & 0 & 0.6116 & 0.4024 & 0.0332 & 0.8812 & 0 & 0 & 1.3191 \\
\hline 1110 & 1.2706 & 0 & 0.1001 & 0.3252 & 0.0155 & 0.8078 & 0 & 0 & 1.0233 \\
\hline
\end{tabular}

times $t_{r_{i-1}}^{i-1}$ and $t_{d_{i-1}}^{i-1}$, whenever $d_{i-1} \leq r_{i-1}$ and $d_{i-1}>r_{i-1}$, respectively. The iterative process and simulation process times with jump are $t_{k+r_{i-1}}^{i-1}$ and $t_{k+d_{i-1}}^{i-1}, \quad i \in I\left(1, K^{*}\right)$, respectively.

7.2. Conceptual Computational Parameter Estimation Scheme. For the conceptual computational dynamic system parameter estimation, we need to introduce a few concepts of local admissible sample/data observation size $m_{k}^{i-1}$ local admissible conditional finite sequence at $t_{k}^{i-1}$ $\in S \mathbb{P}^{i-1}$ and local finite sequence of parameter estimates at $t_{k}^{i-1}$.
Definition 18. For each $i \in I\left(1, K^{*}\right)$, and $t_{k}^{i-1} \in I\left(T_{i-1}-\tau_{i-1}\right.$, $T_{i}$ ), we define local admissible sample/data observation size $m_{k}^{i-1}$ at $t_{k}^{i-1}$ as $m_{k}^{i-1} \in O S_{k}^{i-1}$, where

$O S_{k}^{i-1}= \begin{cases}I\left(2, r_{i-1}+\mathcal{S}_{i-1}+k-1\right), & \text { if } d_{i-1} \leq r_{i-1}, \\ I\left(2, d_{i-1}+\mathcal{S}_{i-1}+k-1\right), & \text { if } d_{i-1}>r_{i-1}, k \in I\left(0, N_{i-1}\right) .\end{cases}$

Moreover, $O S_{k}^{i-1}$ is referred as the local admissible set of lagged sample/data observation size at $t_{k}^{i-1}$. 


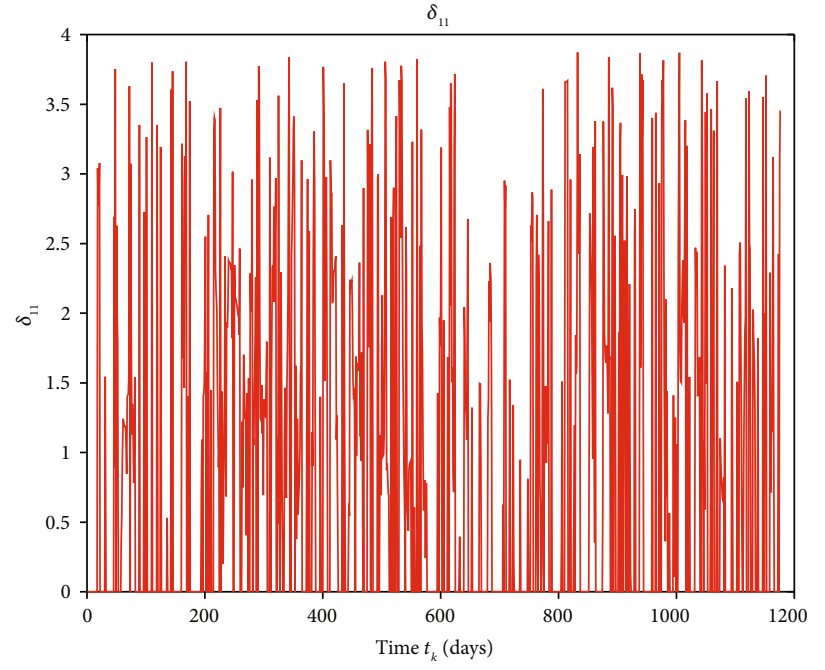

(a)

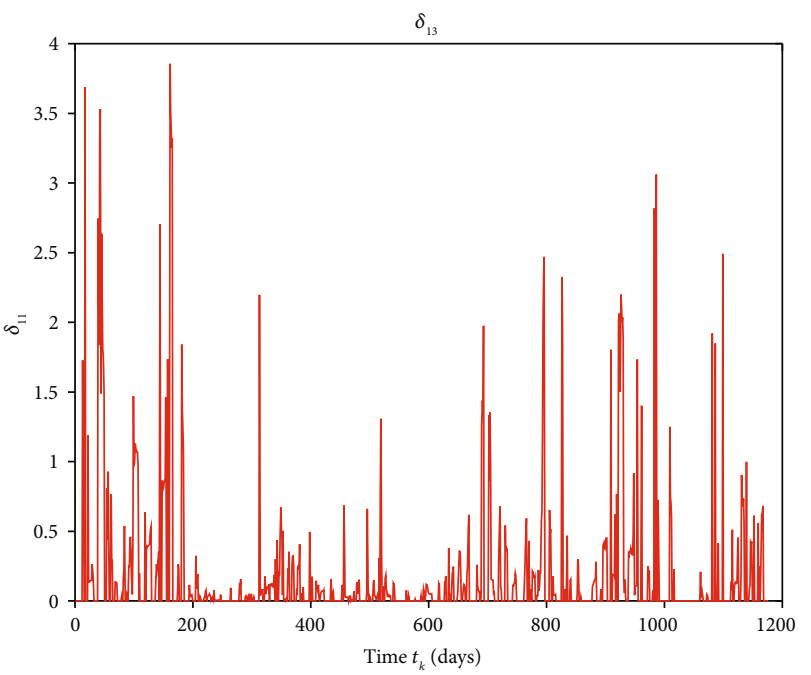

(c)

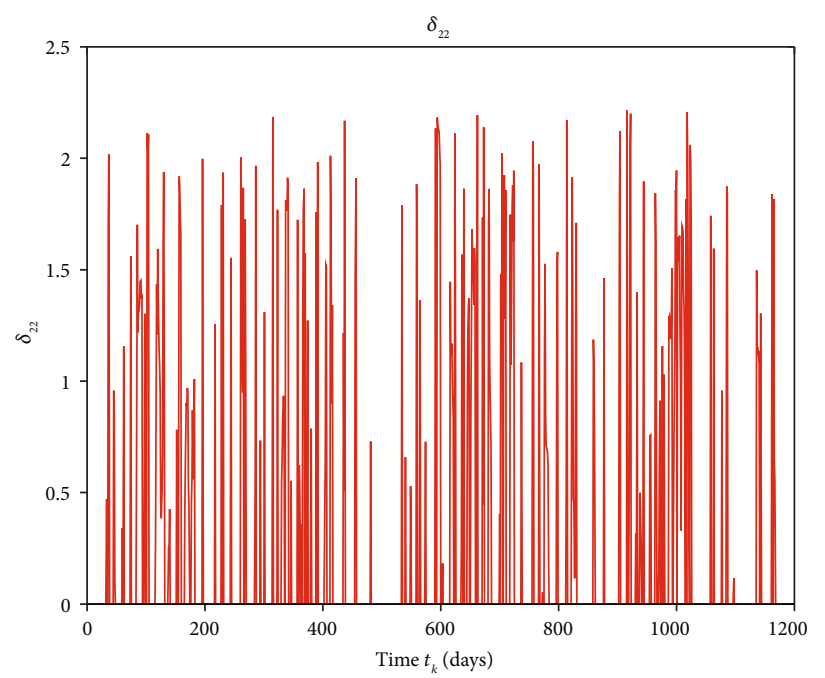

(e)

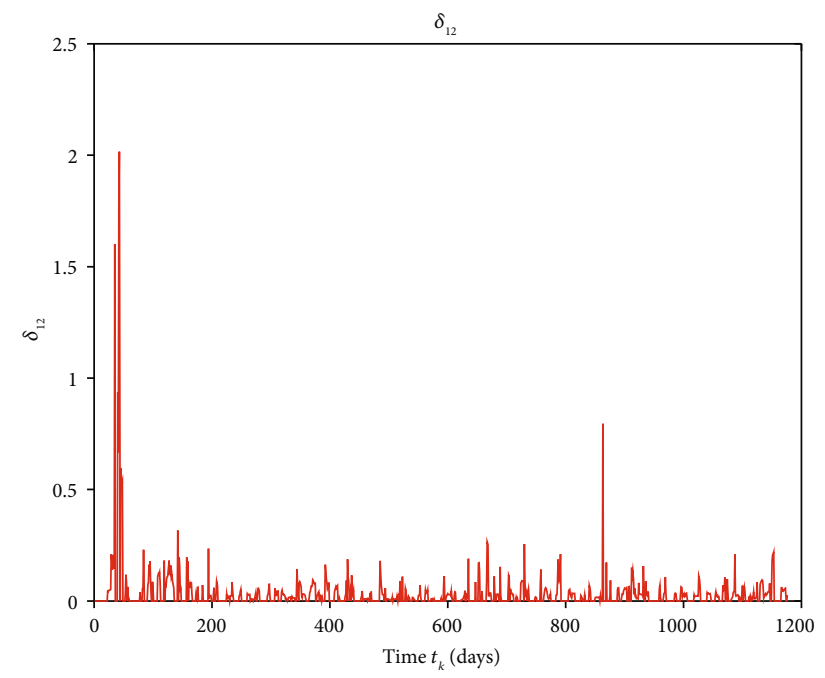

(b)

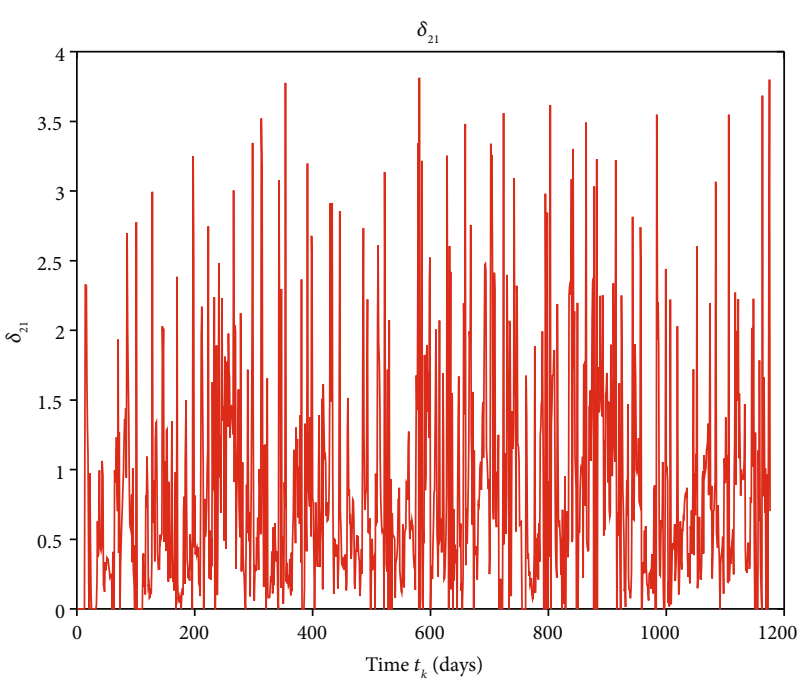

(d)

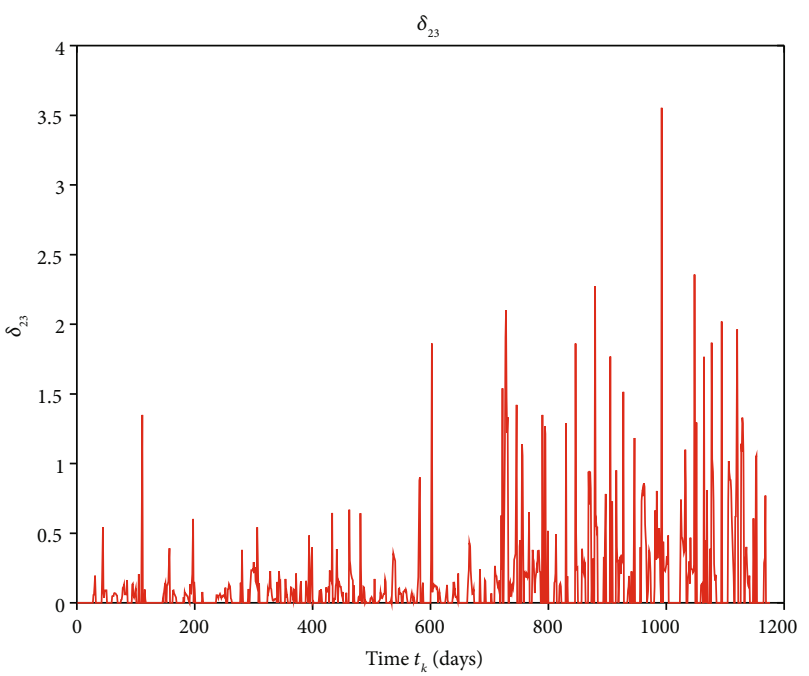

(f)

Figure 10: Continued. 


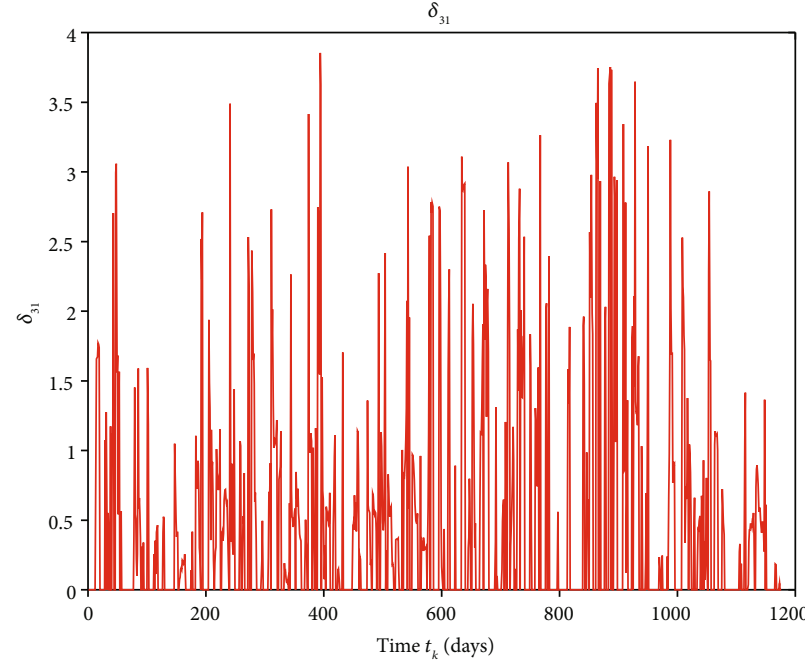

(g)

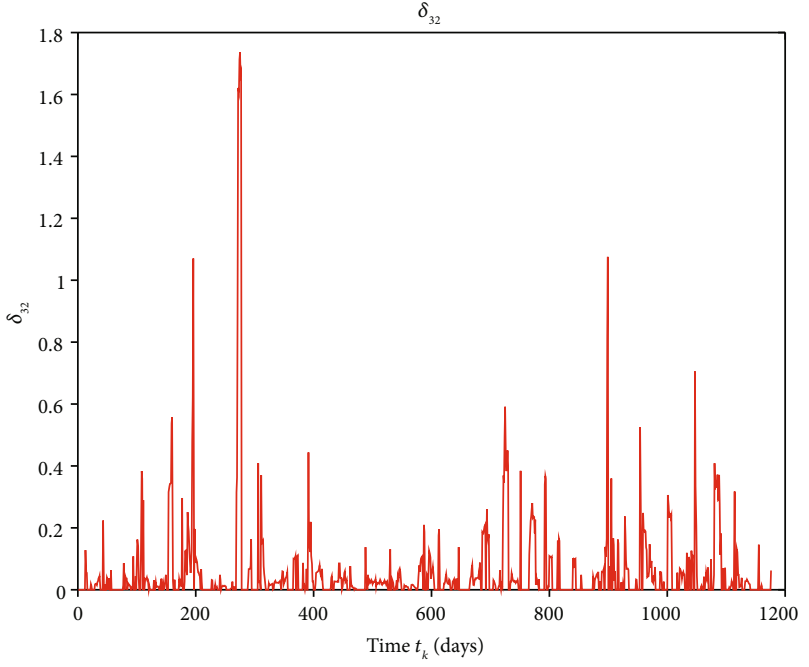

(h)

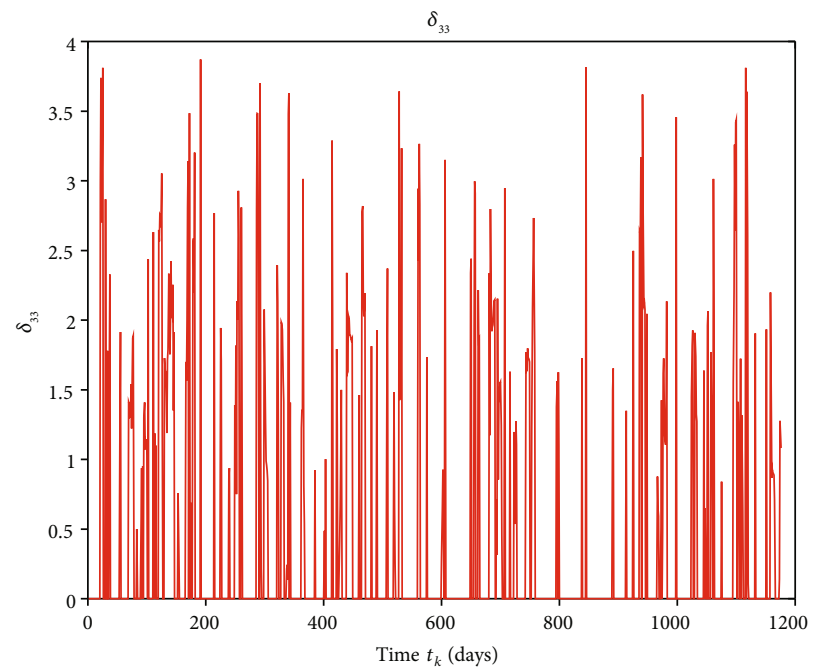

(i)

FIGURE 10: The graph of interaction coefficients $\delta_{1,1}\left(\widehat{m}_{k}, k\right), \delta_{1,2}\left(\widehat{m}_{k}, k\right), \delta_{1,3}\left(\widehat{m}_{k}, k\right), \delta_{2,1}\left(\widehat{m}_{k}, k\right), \delta_{2,2}\left(\widehat{m}_{k}, k\right), \delta_{2,3}\left(\widehat{m}_{k}, k\right), \delta_{3,1}\left(\widehat{m}_{k}, k\right), \delta_{3,2}\left(\widehat{m}_{k}, k\right)$, and $\delta_{3,3}\left(\widehat{m}_{k}, k\right)$.

Remark 19. We note that if $K=0, \mathcal{S}_{i-1}=0$, the point $t_{k}^{i-1}=$ $t_{k} \in\left[t_{0}, T\right]$. Thus, (76) is reduced to

$$
O S_{k}^{i-1}=O S_{k}= \begin{cases}I(2, r+k-1), & \text { if } d \leq r, \\ I(2, d+k-1), & \text { if } d>r, k \in I(0, N)\end{cases}
$$

Definition 20. For each $i \in I\left(1, K^{*}\right), m_{k}^{i-1} \in O S_{k}^{i-1}$ in Definition 18 and $k \in I\left(0, N_{i-1}\right)$, a $m_{k}^{i-1}$ local admissible lagged adapted finite restriction sequence of conditional sample/data observation at time $t_{k}^{i-1}$ to subpartition $P_{k}^{i-1}$ of $\mathbb{P}^{i-1}$ in Definition 8 is defined by $\left(\left\{\mathbb{E}\left[\mathbf{y}^{i-1}\left(t_{l}^{i-1}\right) \mid \mathscr{F}_{l-1}^{i-1}\right]\right\}_{l=k-m_{k}^{i-1}}^{k-1}\right.$,
$\left.\left\{\mathbb{E}\left[\mathbf{p}^{i-1}\left(t_{l}^{i-1}\right) \mid \mathscr{F}_{l-1}^{i-1}\right]\right\}_{l=k-m_{k}^{i-1}}^{k-1}\right)$. Moreover, a $m_{k}^{i-1}$ class of admissible lagged adapted finite sequences of conditional sample/data observation of size $m_{k}^{i-1}$ at $t_{k}^{i-1}$ is defined by

$$
\mathscr{A}_{k}^{i-1}=\left\{\begin{array}{l}
\left\{\left\{\mathbb{E}\left[\mathbf{y}^{i-1}\left(t_{l}^{i-1}\right) \mid \mathscr{F}_{l-1}^{i-1}\right]\right\}_{l=k-m_{k}^{i-1}}^{k-1}\right\}_{m_{k}^{i-1} \in O S_{k}^{i-1}}, \\
\left\{\left\{\mathbb{E}\left[\mathbf{p}^{i-1}\left(t_{l}^{i-1}\right) \mid \mathscr{F}_{l-1}^{i-1}\right]\right\}_{l=k-m_{k}^{i-1}}^{k-1}\right\}_{m_{k}^{i-1} \in O S_{k}^{i-1}}
\end{array}\right.
$$

In the case of energy commodity model, for each $i \epsilon$ $I\left(1, K^{*}\right), m_{k}^{i-1} \in O S_{k}^{i-1}$, we find corresponding $m_{k}^{i-1}$ local 


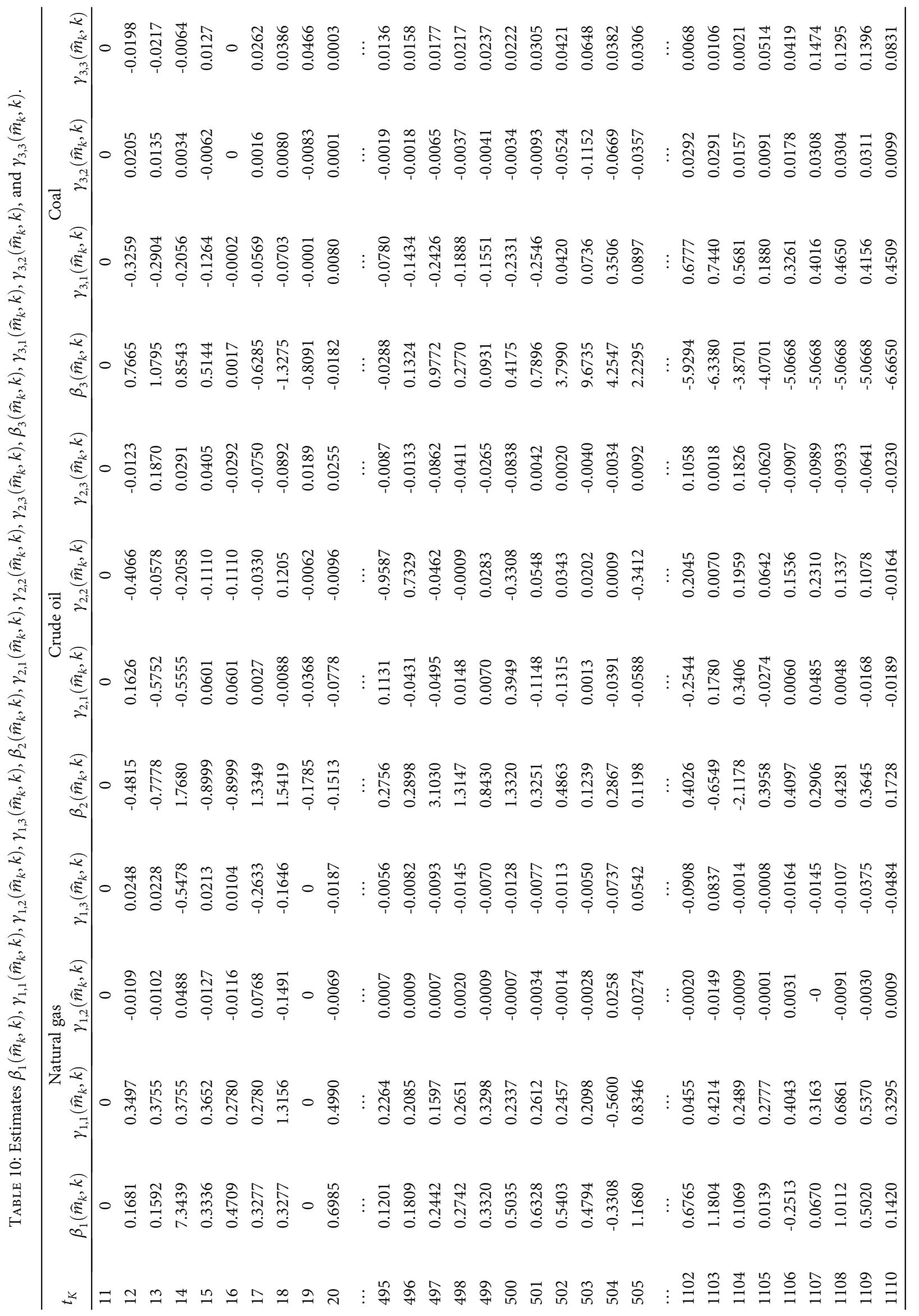




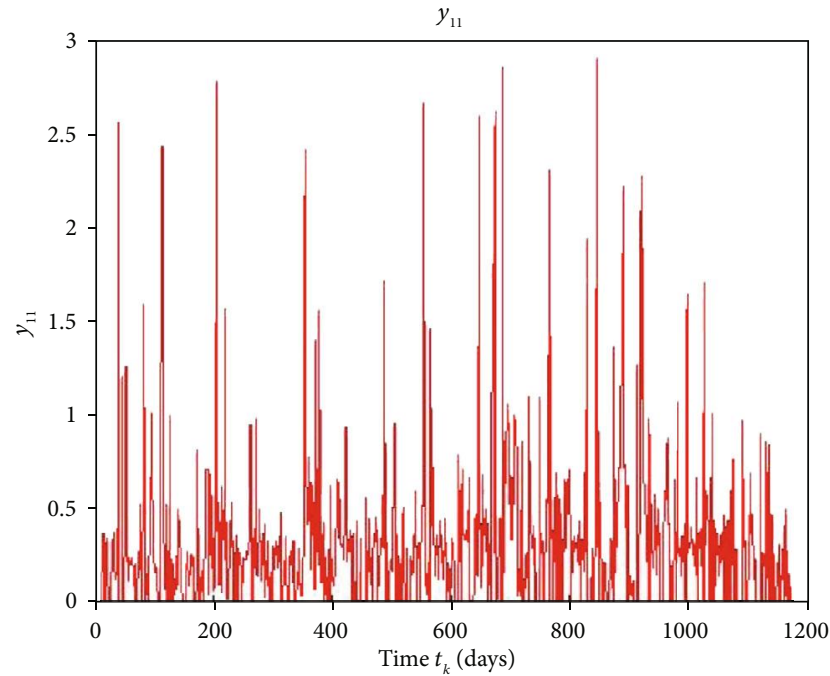

(a)

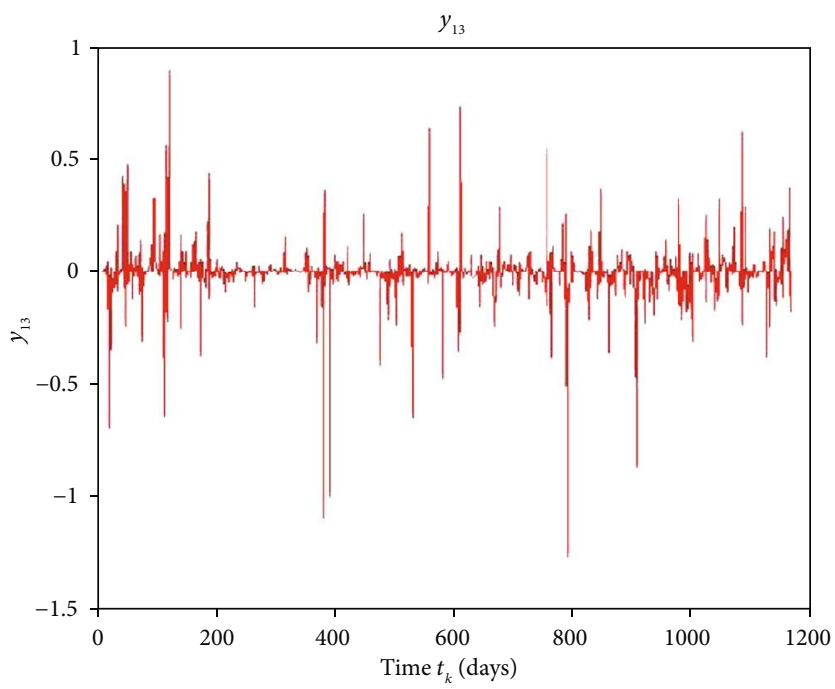

(c)

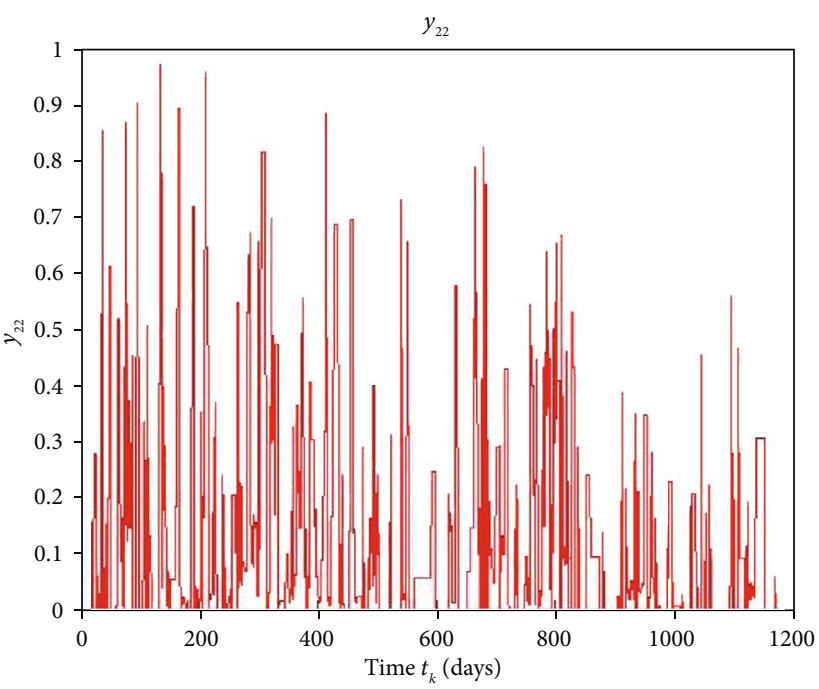

(e)

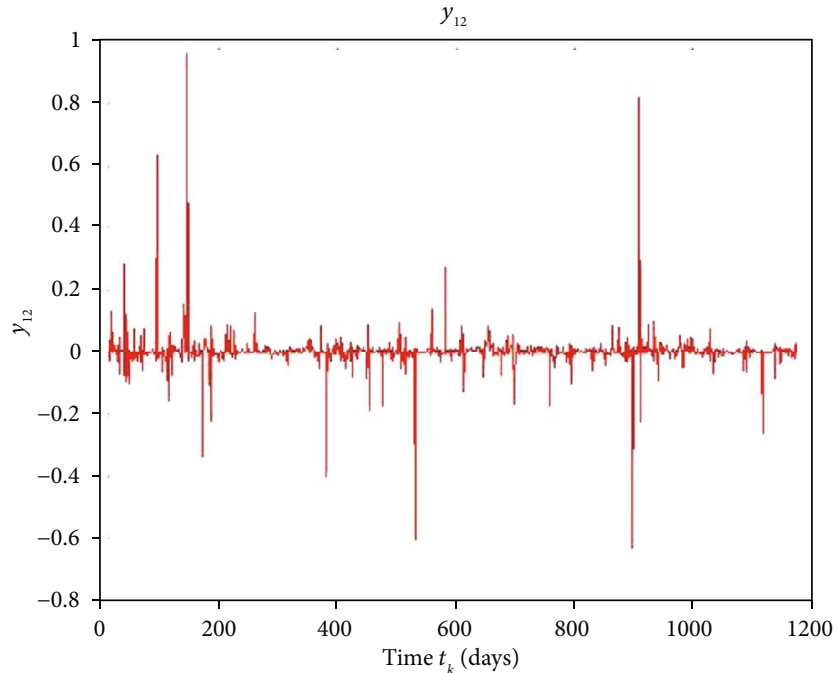

(b)

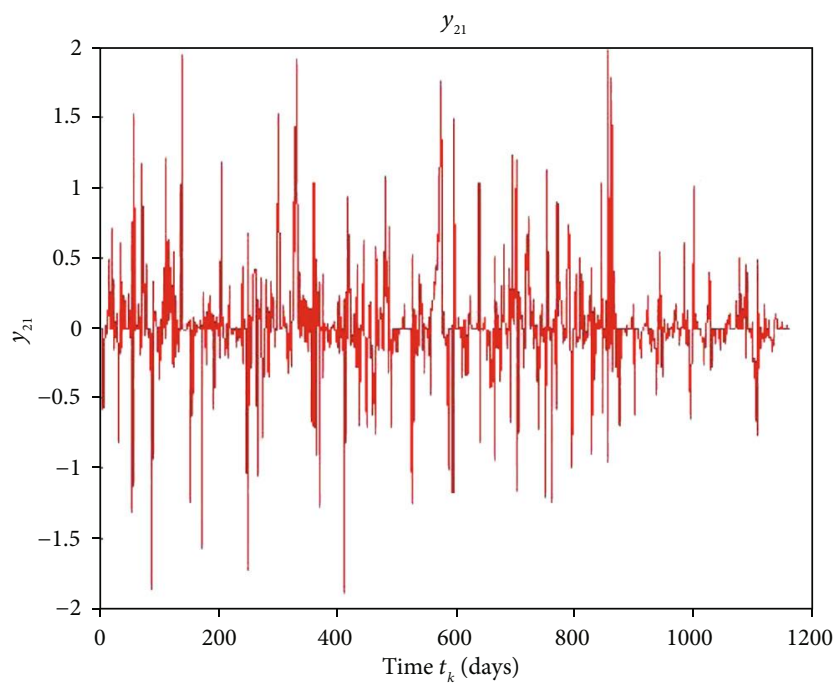

(d)

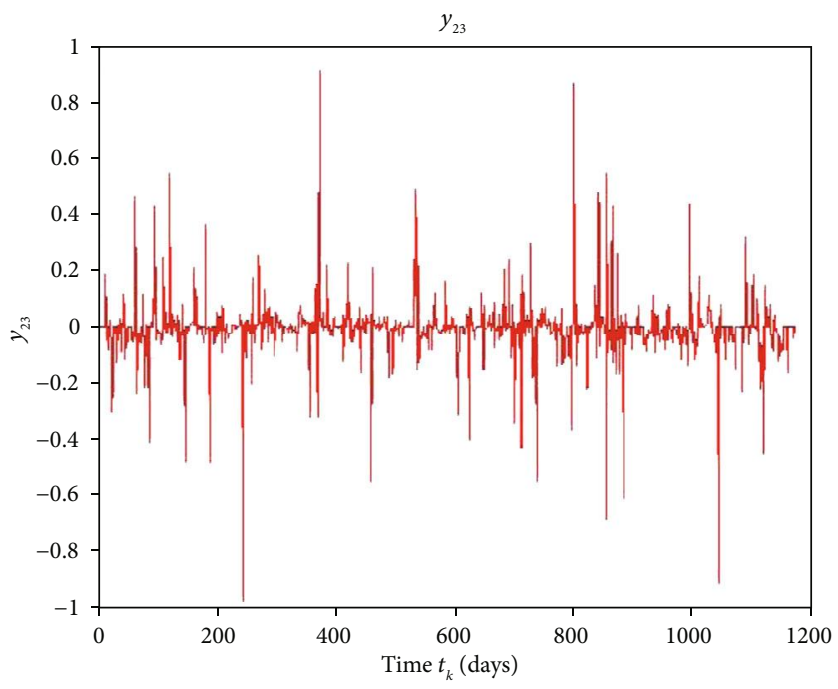

(f)

Figure 11: Continued. 


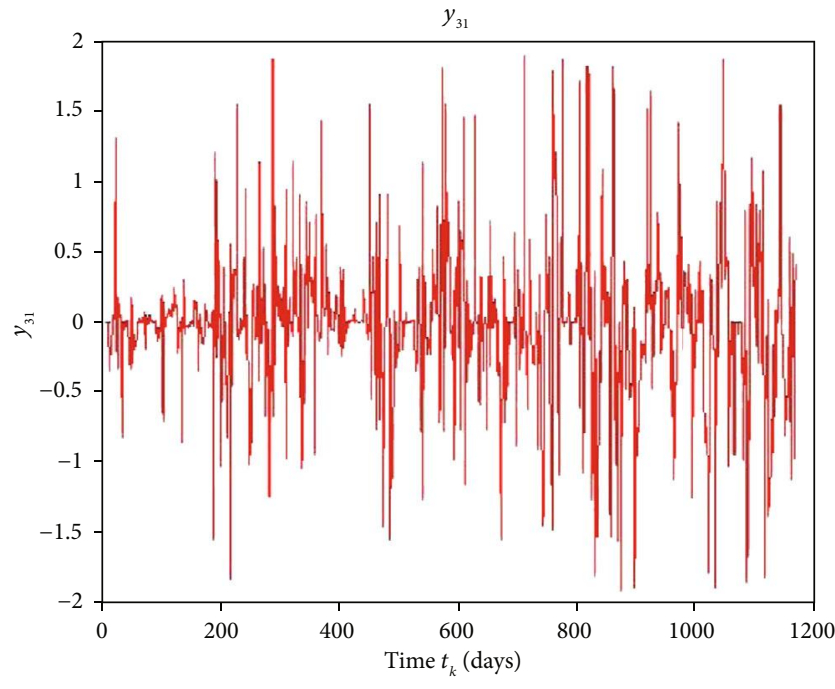

(g)

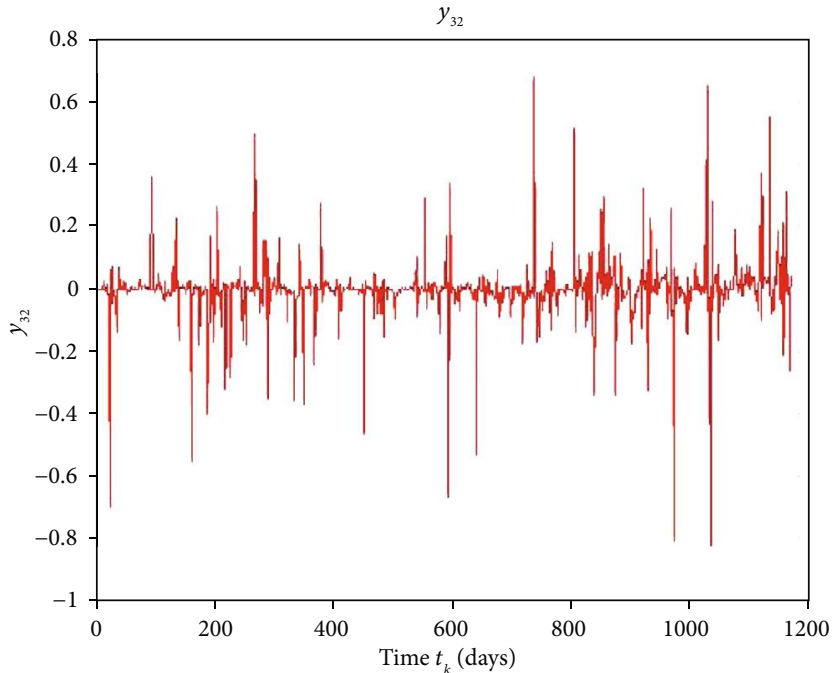

(h)

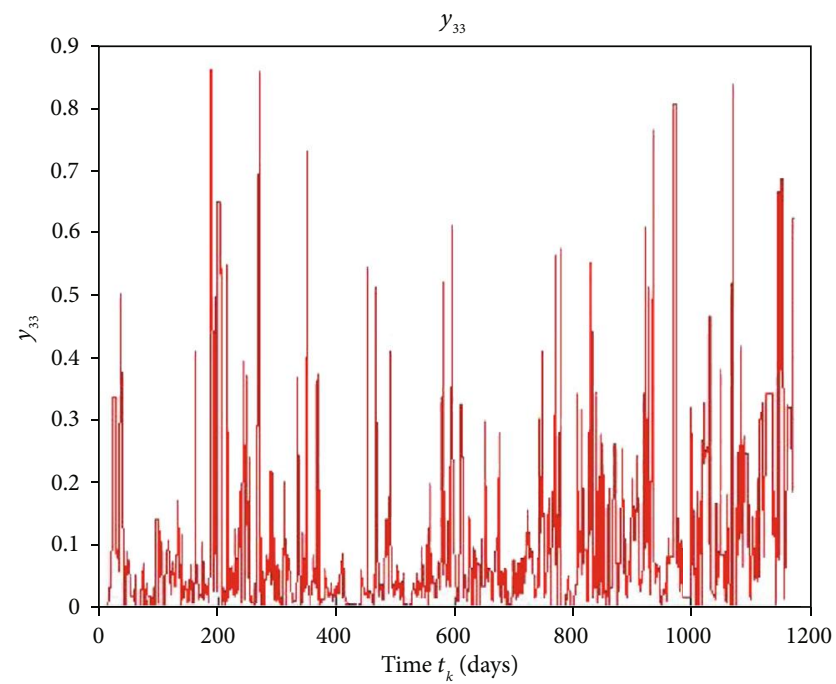

(i)

FIGURE 11: The graph of interaction coefficients $\gamma_{1,1}\left(\widehat{m}_{k}, k\right), \gamma_{1,2}\left(\widehat{m}_{k}, k\right), \gamma_{1,3}\left(\widehat{m}_{k}, k\right), \gamma_{2,1}\left(\widehat{m}_{k}, k\right), \gamma_{2,2}\left(\widehat{m}_{k}, k\right), \gamma_{2,3}\left(\widehat{m}_{k}, k\right), \gamma_{3,1}\left(\widehat{m}_{k}, k\right), \gamma_{3,2}\left(\widehat{m}_{k}, k\right)$, and $\gamma_{3,3}\left(\hat{m}_{k}, k\right)$.

admissible adapted finite sequence of conditional sample/data observation at $t_{k}^{i-1},\left(\left\{\mathbb{E}\left[\mathbf{y}^{i-1}\left(t_{l}^{i-1}\right) \mid \mathscr{F}_{l-1}^{i-1}\right]\right\}_{l=k-m_{k}^{i-1}}^{k-1}\right.$
$\left.\left\{\mathbb{E}\left[\mathbf{p}^{i-1}\left(t_{l}^{i-1}\right) \mid \mathscr{F}_{l-1}^{i-1}\right]\right\}_{l=k-m_{k}^{i-1}}^{k-1}\right)$. For $i \in I\left(1, K^{*}\right)$, using this sequence and solutions of (70), we compute

$$
\left\{u_{j}^{i-1}\left(m_{k}, t_{k}^{i-1}\right), \beta_{j}^{i-1}\left(m_{k}^{i-1}, t_{k}^{i-1}\right), \kappa_{j, l}^{i-1}\left(m_{k}^{i-1}, t_{k}^{i-1}\right), \gamma_{j, l}^{i-1}\left(m_{k}^{i-1}, t_{k}^{i-1}\right), \delta_{j, l}^{i-1}\left(m_{k}^{i-1}, t_{k}^{i-1}\right), \sigma_{j, l}^{i-1}\left(m_{k}^{i-1}, t_{k}^{i-1}\right), k \in\left[0, N_{i-1}\right]\right.
$$




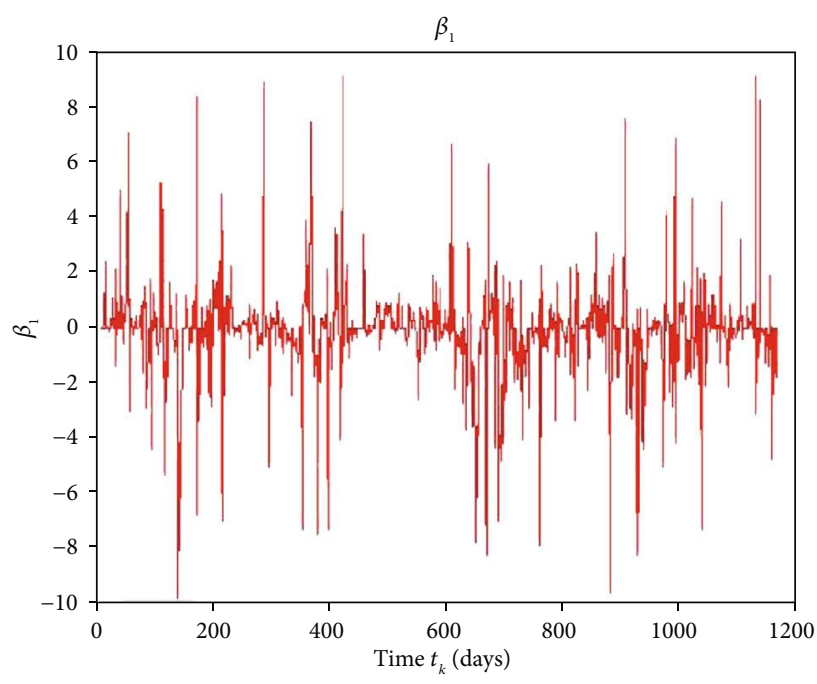

(a)

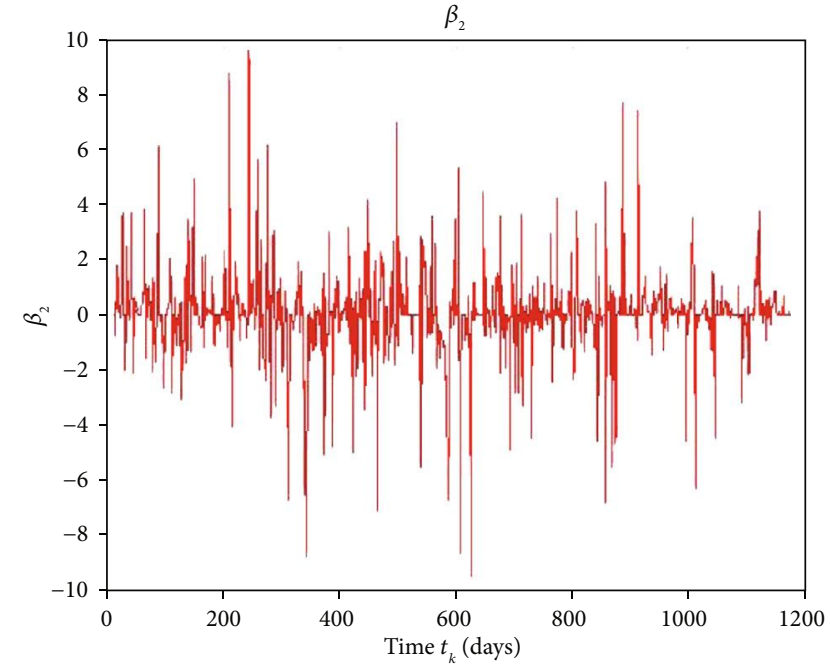

(b)

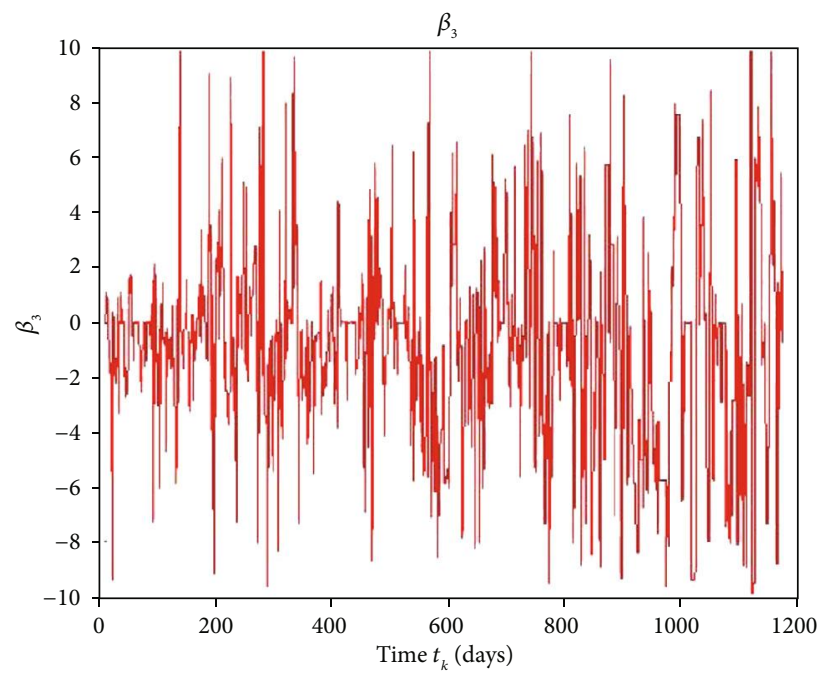

(c)

FigURE 12: The graph of $\beta_{1}\left(\widehat{m}_{k}, k\right), \beta_{2}\left(\widehat{m}_{k}, k\right)$, and $\beta_{3}\left(\widehat{m}_{k}, k\right)$ for natural gas, crude oil, and coal, respectively.

for $j, l \in I(1, n)$. This leads to a local finite sequence of parameter estimates at $t_{k}^{i-1}$ defined on $O S_{k}^{i-1}$ as follows:

$$
\left\{\left(\widehat{u}_{j}^{i-1}\left(m_{k}^{i-1}, t_{k}^{i-1}\right), \widehat{\beta}_{j}^{i-1}\left(m_{k}^{i-1}, t_{k}^{i-1}\right), \widehat{\kappa}_{j, l}^{i-1}\left(m_{k}^{i-1}, t_{k}^{i-1}\right), \widehat{\gamma}_{j, l}^{i-1}\left(m_{k}^{i-1}, t_{k}^{i-1}\right), \widehat{\delta}_{j, l}^{i-1}\left(m_{k}^{i-1}, t_{k}^{i-1}\right), \widehat{\sigma}_{j, l}^{i-1}\left(m_{k}^{i-1}, t_{k}^{i-1}\right)\right)\right\}_{m_{k}^{i-1} \in O S_{k}^{i-1}} .
$$

The above defined collection is denoted by

$$
\left(\boldsymbol{U}_{k}, \mathscr{B}_{k}, \mathscr{K}_{k}, \gamma_{k}, \boldsymbol{\delta}_{k}, \boldsymbol{\sigma}_{k}\right)=\left\{\begin{array}{l}
\left\{\left(\widehat{u}_{j}^{i-1}\left(m_{k}^{i-1}, t_{k}^{i-1}\right), \widehat{\beta}_{j}^{i-1}\left(m_{k}^{i-1}, t_{k}^{i-1}\right), \widehat{\kappa}_{j, l}^{i-1}\left(m_{k}^{i-1}, t_{k}^{i-1}\right), \widehat{\gamma}_{j, l}^{i-1}\left(m_{k}^{i-1}, t_{k}^{i-1}\right), \widehat{\delta}_{j, l}^{i-1}\left(m_{k}^{i-1}, t_{k}^{i-1}\right), \widehat{\sigma}_{j, l}^{i-1}\left(m_{k}^{i-1}, t_{k}^{i-1}\right)\right)\right\}_{m_{k}^{i-1} \in O S_{k}^{i-1}}, \\
\text { for } j \in I(1, n), i \in I\left(1, K^{*}\right) .
\end{array}\right.
$$


7.3. Conceptual Computation of State Simulation Scheme. For the development of a conceptual computational scheme, we need to employ the method of induction. The presented simulation scheme is based on the idea of lagged adaptive expectation process [17]. For $j, l \in I(1, n)$, an autocorrelation function (ACF) analysis $[21,32]$ performed on $\left(s_{m_{k}^{i-1}, k}^{j, j}(\mathbf{y})\right.$, $\left.s_{m_{k}^{i-1, k}}^{j, j}(\mathbf{p})\right)$ suggests that the interconnected discrete-time dynamic model of local conditional sample mean and sample variance statistics in (48) is of order $d_{i-1}=2$. In view of this, we need to identify the initial data. We begin with a given initial data $\left(\mathbf{y}^{i-1}\left(T_{i-1}\right), \mathbf{p}^{i-1}\left(T_{i-1}\right)\right),\left(\left\{\sum_{m_{0}^{i-1}, t_{0}^{i-1}}(y)\right\}_{m_{0}^{i-1} \in O S_{0}^{i-1}}\right.$, $\left.\left\{\sum_{m_{0}^{i-1}, t_{0}^{i-1}}(p)\right\}_{m_{0}^{i-1} \in O S_{0}^{i-1}}\right),\left(\left\{\sum_{m_{-1}^{i-1}, t_{-1}^{i-1}}(y)\right\}_{m_{-1}^{i-1} \in O S_{-1}^{i-1}},\left\{\sum_{m_{-1}^{i-1}, t_{-1}^{i-1}}(p)\right\}\right.$ $\left.m_{-1}^{i-1} \in O S_{-1}^{i-1}\right), \quad\left(\left\{\bar{S}_{m_{-1}^{i-1}, t_{-1}^{i-1}}(y)\right\}_{m_{-1}^{i-1} \in O S_{-1}^{i-1}},\left\{\bar{S}_{m_{-1}^{i-1}, t_{-1}^{i-1}}(p)\right\}_{m_{-1}^{i-1} \in O S_{-1}^{i-1}}\right)$ as defined in (45), (46), (47), and (48).

Let $\quad\left(\mathbf{y}^{s}\left(m_{k}^{i-1}, t_{k}^{i-1}\right), \mathbf{p}^{s}\left(m_{k}^{i-1}, t_{k}^{i-1}\right)\right) \equiv\left(\mathbf{y}^{i-1, s}\left(m_{k}^{i-1}, t_{k}^{i-1}\right)\right.$, $\left.\mathbf{p}^{i-1, s}\left(m_{k}^{i-1}, t_{k}^{i-1}\right)\right)$ be a simulated value of $\left(\mathbb{E}\left[\mathbf{y}^{i-1}\left(t_{k}^{i-1}\right) \mid \mathscr{F}_{k-1}^{i-1}\right]\right.$, $\left.\mathbb{E}\left[\mathbf{p}^{i-1}\left(t_{k}^{i-1}\right) \mid \mathscr{F}_{k-1}^{i-1}\right]\right)$ at time $t_{k}^{i-1}$ corresponding to an admissible sequence $\left\{\mathbb{E}\left[\mathbf{y}^{i-1}\left(t_{l}^{i-1}\right) \mid \mathscr{F}_{l-1}^{i-1}\right], \mathbb{E}\left[\mathbf{p}^{i-1}\left(t_{l}^{i-1}\right) \mid \mathscr{F}_{l-1}^{i-1}\right]\right\}_{l=k-m_{k}}^{k-1} \epsilon$ $\mathscr{A} \boldsymbol{S}_{k}^{i-1}$. For $q=1$, and $j \in \boldsymbol{I}(1, n)$, the simulated value $\left(y_{j}^{s}\left(m_{k}^{i-1}, t_{k}^{i-1}\right), p_{j}^{s}\left(m_{k}^{i-1}, t_{k}^{i-1}\right)\right)$ is generated from the discretized Euler scheme (62) as follows:

$$
\left\{\begin{aligned}
y_{j}^{s}\left(m_{k}^{i-1}, t_{k}^{i-1}\right) & =y_{j}^{s}\left(m_{k-1}^{i-1}, t_{k-1}^{i-1}\right)+\left(u_{j}^{i-1}\left(m_{k-1}^{i-1}, t_{k-1}^{i-1}\right)-y_{j}^{s}\left(m_{k-1}^{i-1}, t_{k-1}^{i-1}\right)\right)\left[\kappa_{j, j}^{i-1}\left(m_{k-1}^{i-1}, t_{k-1}^{i-1}\right) y_{j}^{s}\left(m_{k-1}^{i-1}, t_{k-1}^{i-1}\right)+\sum_{l \neq j}^{n} \kappa_{j, l}^{i-1}\left(m_{k-1}^{i-1}, t_{k-1}^{i-1}\right) y_{l}^{s}\left(m_{k-1}^{i-1}, t_{k-1}^{i-1}\right)\right] \Delta t \\
& +\left(u_{j}^{i-1}\left(m_{k-1}^{i-1}, t_{k-1}^{i-1}\right)-y_{j}^{s}\left(m_{k-1}^{i-1}, t_{k-1}^{i-1}\right)\right)\left[\delta_{j, j}^{i-1}\left(m_{k-1}^{i-1}, t_{k-1}^{i-1}\right) \Delta W_{j, j}\left(m_{k}^{i-1}, t_{k}^{i-1}\right)+\sum_{l \neq j}^{n} \delta_{j, l}^{i-1}\left(m_{k-1}^{i-1}, t_{k-1}^{i-1}\right) y_{l}^{s}\left(m_{k-1}^{i-1}, t_{k-1}^{i-1}\right) \Delta W_{j, l}\left(m_{k}^{i-1}, t_{k}^{i-1}\right)\right] \\
& \cdot t_{k}^{i-1} \in\left[T_{i-1}, T_{i}\right), \\
p_{j}^{s}\left(m_{k}^{i-1}, t_{k}^{i-1}\right) & =p_{j}^{s}\left[\gamma_{j, j}^{i-1}\left(m_{k-1}^{i-1}, t_{k-1}^{i-1}\right)\left(y_{j}^{s}\left(m_{k-1}^{i-1}, t_{k-1}^{i-1}\right)-p_{j}^{s}\left(m_{k-1}^{i-1}, t_{k-1}^{i-1}\right)\right)+\beta_{j}^{i-1}\left(m_{k-1}^{i-1}, t_{k-1}^{i-1}\right)+\sum_{l \neq j}^{n} \gamma_{j, l}^{i-1}\left(m_{k-1}^{i-1}, t_{k-1}^{i-1}\right) p_{l}^{s}\left(m_{k-1}^{i-1}, t_{k-1}^{i-1}\right)\right] \Delta t \\
& +\sigma_{j, j}^{i-1}\left(m_{k-1}^{i-1}, t_{k-1}^{i-1}\right) p_{j}^{s}\left(m_{k-1}^{i-1}, t_{k-1}^{i-1}\right) \Delta Z_{j, j}\left(m_{k}^{i-1}, t_{k}^{i-1}\right)+p_{j}^{s}\left(m_{k-1}^{i-1}, t_{k-1}^{i-1}\right) \sum_{l \neq j}^{n} \sigma_{j, l}^{i-1}\left(m_{k-1}^{i-1}, t_{k-1}^{i-1}\right) p_{l}^{s}\left(m_{k-1}^{i-1}, t_{k-1}^{i-1}\right) \Delta Z_{j, l}\left(m_{k}^{i-1}, t_{k}^{i-1}\right), \\
& \cdot t_{k}^{i-1} \in\left[T_{i-1}, T_{i}\right) .
\end{aligned}\right.
$$

To find the simulated value $y_{j}^{i, s}\left(T_{i}\right)$ and $p_{j}^{i, s}\left(T_{i}\right)$, we need to estimate $\widehat{\pi}_{j}^{i}$ and $\widehat{\theta}_{j}^{i}$ by first simulating

$$
\lim _{t \rightarrow T_{i}^{-}} y_{j}^{i-1}\left(t, T_{i-1}, \mathbf{y}^{i-1, s}\right) \equiv y_{j}^{s}\left(m_{N_{i-1}}^{i-1}, t_{N_{i-1}}^{i-1}\right) \quad \text { and } \quad \lim _{t \rightarrow T_{i}^{-}} p_{j}^{i-1}
$$

$\left(t, T_{i-1}, \mathbf{y}^{i-1, s}, \mathbf{p}^{i-1, s}\right) \equiv p_{j}^{s}\left(m_{N_{i-1}}^{i-1}, t_{N_{i-1}}^{i-1}\right)$ as follows:

$$
\begin{aligned}
y_{j}^{s}\left(m_{N_{i-1}}^{i-1}, t_{N_{i-1}}^{i-1}\right)= & y_{j}^{s}\left(m_{N_{i-1}-1}^{i-1}, t_{N_{i-1}-1}^{i-1}\right)+\left(u_{j}^{i-1}\left(m_{N_{i-1}-1}^{i-1}, i_{N_{i-1}-1}^{i-1}\right)-y_{j}^{s}\left(m_{N_{i-1}-1}^{i-1}, t_{N_{i-1}-1}^{i-1}\right)\right)\left[\sum_{l=1}^{n} \kappa_{j, l}^{i-1}\left(m_{N_{i-1}-1}^{i-1}, t_{N_{i-1}-1}^{i-1}\right) y_{l}^{s}\left(m_{N_{i-1}-1}^{i-1}, t_{N_{i-1}-1}^{i-1}\right)\right] \Delta t \\
& +\left(u_{j}^{i-1}\left(m_{N_{i-1}-1}^{i-1}, t_{N_{i-1}-1}^{i-1}\right)-y_{j}^{s}\left(m_{N_{i-1}-1}^{i-1}, t_{N_{i-1}-1}^{i-1}\right)\right)\left[\delta_{j, j}^{i-1}\left(m_{N_{i-1}-1}^{i-1}, t_{N_{i-1}-1}^{i-1}\right) \Delta W_{j, j}\left(m_{N_{i-1}}^{i-1}, t_{N_{i-1}}^{i-1}\right)\right. \\
& \left.+\sum_{l \neq j}^{n} \delta_{j, l}^{i-1}\left(m_{N_{i-1}-1}^{i-1}, t_{N_{i-1}-1}^{i-1}\right) y_{l}^{s}\left(m_{N_{i-1}-1}^{i-1}, t_{N_{i-1}-1}^{i-1}\right) \Delta W_{j, l}\left(m_{N_{i-1}}^{i-1}, t_{N_{i-1}}^{i-1}\right)\right] \\
p_{j}^{s}\left(m_{N_{i-1}}^{i-1}, t_{N_{i-1}}^{i-1}\right)= & p_{j}^{s}\left[\gamma_{j, j}^{i-1}\left(m_{N_{i-1}-1}^{i-1}, t_{N_{i-1}-1}^{i-1}\right)\left(y_{j}^{s}\left(m_{N_{i-1}-1}^{i-1}, t_{N_{i-1}-1}^{i-1}\right)-p_{j}^{s}\left(m_{N_{i-1}-1}^{i-1}, t_{N_{i-1}-1}^{i-1}\right)\right)+\beta_{j}^{i-1}\left(m_{N_{i-1}-1}^{i-1}, t_{N_{i-1}-1}^{i-1}\right)\right. \\
& \left.+\sum_{l \neq j}^{n} \gamma_{j, l}^{i-1}\left(m_{N_{i-1}-1}^{i-1}, t_{N_{i-1}-1}^{i-1}\right) p_{l}^{s}\left(m_{N_{i-1}-1}^{i-1}, t_{N_{i-1}-1}^{i-1}\right)\right] \Delta t+\sigma_{j, j}^{i-1}\left(m_{N_{i-1}-1}^{i-1}, t_{N_{i-1}-1}^{i-1}\right) p_{j}^{s}\left(m_{N_{i-1}-1}^{i-1}, t_{N_{i-1}-1}^{i-1}\right) \Delta Z_{j, j}\left(m_{N_{i-1}}^{i-1}, t_{N_{i-1}}^{i-1}\right) \\
& +p_{j}^{s}\left(m_{N_{i-1}-1}^{i-1}, t_{N_{i-1}-1}^{i-1}\right) \sum_{l \neq j}^{n} \sigma_{j, l}^{i-1}\left(m_{N_{i-1}-1}^{i-1}, t_{N_{i-1}-1}^{i-1}\right) p_{l}^{s}\left(m_{N_{i-1}-1}^{i-1}, t_{N_{i-1}-1}^{i-1}\right) \Delta Z_{j, l}\left(m_{N_{i-1}}^{i-1}, t_{N_{i-1}}^{i-1}\right) .
\end{aligned}
$$


From this, we calculate $\widehat{\pi}_{j}^{i}$ and $\widehat{\theta}_{j}^{i}$ as:

$$
\begin{gathered}
\widehat{\pi}_{j}^{i}=\frac{\mathbb{E}\left[y_{j}^{i-1}\left(T_{i}\right) \mid \mathscr{F}_{T_{i}-1}^{i-1}\right]}{y_{j}^{s}\left(m_{N_{i-1}}^{i-1}, t_{N_{i-1}}^{i-1}\right)} \\
\widehat{\theta}_{j}^{i}=\frac{\mathbb{E}\left[p_{j}^{i-1}\left(T_{i}\right) \mid \mathscr{F}_{T_{i}-1}^{i-1}\right]}{p_{j}^{s}\left(m_{N_{i-1}}^{i-1}, t_{N_{i-1}}^{i-1}\right)} .
\end{gathered}
$$

Thus, $y_{j}^{i, s}\left(T_{i}\right)=\widehat{\pi}_{j}^{i} y_{j}^{i-1, s}\left(T_{i}^{-}, T_{i-1}, \mathbf{y}^{i-1, s}\right)$ and $p_{j}^{i, s}\left(T_{i}\right)=\widehat{\theta}_{j}^{i}$ $p_{j}^{i-1, s}\left(T_{i}^{-}, T_{i-1}, \mathbf{y}^{i-1, s}, \mathbf{p}^{i-1, s}\right)$. Let $\quad\left(\left\{\mathbf{y}^{s}\left(m_{k}^{i-1}, t_{k}^{i-1}\right)\right\}_{m_{k}^{i-1} \in O S_{k}^{i-1}}\right.$, $\left.\left\{\mathbf{p}^{s}\left(m_{k}^{i-1}, t_{k}^{i-1}\right)\right\}_{m_{k}^{i-1} \in O S_{k}^{i-1}}\right)$ be a $m_{k}^{i-1}$ - local sequence of simulated values corresponding to $m_{k}^{i-1}$-admissible lagged adapted finite sequence of conditional observation belonging to $\mathscr{A} \mathcal{S}_{k}^{i-1}$, and corresponding term of sequence.
$\left(\boldsymbol{U}_{k}, \mathscr{B}_{k}, \mathscr{K}_{k}, \boldsymbol{\gamma}_{k}, \boldsymbol{\delta}_{k}, \boldsymbol{\sigma}_{k}\right)$. Thus, for each $i \in I\left(1, K^{*}\right)$, $\left(\left\{\mathbf{y}^{s}\left(m_{k}^{i-1}, t_{k}^{i-1}\right)\right\}_{m_{k}^{i-1} \in O S_{k}^{i-1}}, \quad\left\{\mathbf{p}^{s}\left(m_{k}^{i-1}, t_{k}^{i-1}\right)\right\}_{m_{k}^{i-1} \in O S_{k}^{i-1}}\right)$ are the finite sequence correspondence of simulated values of $\left(\mathbb{E}\left[\mathbf{y}^{i-1}\left(t_{k}^{i-1}\right) \mid \mathscr{F}_{k-1}^{i-1}\right], \mathbb{E}\left[\mathbf{p}^{i-1}\left(t_{k}^{i-1}\right) \mid \mathscr{F}_{k-1}^{i-1}\right]\right)$ at $t_{k}^{i-1}$.

7.4. Mean-Square Suboptimal Procedure. To find the best estimate of $\left(\mathbb{E}\left[y\left(t_{k}^{i-1}\right) \mid \mathscr{F}_{k-1}^{i-1}\right], \mathbb{E}\left[\mathbf{p}\left(t_{k}^{i-1}\right) \mid \mathscr{F}_{k-1}^{i-1}\right]\right)$ using a local admissible finite sequence $\left(\left\{\mathbf{y}^{s}\left(m_{k}^{i-1}, t_{k}^{i-1}\right)\right\}_{m_{k}^{i-1} \in O S_{k}^{i-1}}\right.$, $\left.\left\{\mathbf{p}^{s}\left(m_{k}^{i-1}, t_{k}^{i-1}\right)\right\}_{m_{k}^{i-1} \in O S_{k}^{i-1}}\right)$, we need to compute a finite sequence of quadratic mean square error corresponding to $\left(\left\{\mathbf{y}^{s}\left(m_{k}^{i-1}, t_{k}^{i-1}\right)\right\}_{m_{k}^{i-1} \in O S_{k}^{i-1}},\left\{\mathbf{p}^{s}\left(\mathrm{~m}_{k}^{i-1}, t_{k}^{i-1}\right)\right\}_{m_{k}^{i-1} \in O S_{k}^{i-1}}\right)$. The quadratic mean square error is defined below.

Definition 21. For each $i \in I\left(1, K^{*}\right)$, the quadratic mean square error of $\left(\mathbb{E}\left[\mathbf{y}\left(t_{k}^{i-1}\right) \mid \mathscr{F}_{k-1}^{i-1}\right], \mathbb{E}\left[\mathbf{p}\left(t_{k}^{i-1}\right) \mid \mathscr{F}_{k-1}^{i-1}\right]\right)$ relative to each member of the term of local admissible sequence $\left(\left\{\mathbf{y}^{s}\left(m_{k}^{i-1}, t_{k}^{i-1}\right)\right\}_{m_{k}^{i-1} \in O S_{k}^{i-1}},\left\{\mathbf{p}^{s}\left(m_{k}^{i-1}, t_{k}^{i-1}\right)\right\}_{m_{k}^{i-1} \in O S_{k}^{i-1}}\right)$ of simulated values is defined by

$$
\left\{\Xi_{m_{k}^{i-1}, t_{k}^{i-1}}=\left\|\mathbf{y}^{s}\left(m_{k}^{i-1}, t_{k}^{i-1}\right)-\mathbb{E}\left[\mathbf{y}^{i-1}\left(t_{k}^{i-1}\right) \mid \mathscr{F}_{k-1}^{i-1}\right]\right\|^{2}+\left\|\mathbf{p}^{s}\left(m_{k}^{i-1}, t_{k}^{i-1}\right)-\mathbb{E}\left[\mathbf{p}^{i-1}\left(t_{k}^{i-1}\right) \mid \mathscr{F}_{k-1}^{i-1}\right]\right\|^{2}\right.
$$

For any arbitrary small positive number $\varepsilon$ and for each time $t_{k}^{i-1}$, to find the the best estimate from the admissible simulated values of simulated sequence of $\left(\left\{\mathbf{y}^{s}\left(m_{k}^{i-1}, t_{k}^{i-1}\right)\right\}_{m_{k}^{i-1} \in O S_{k}^{i-1}},\left\{\mathbf{p}^{s}\left(\mathrm{~m}_{k}^{i-1}, t_{k}^{i-1}\right)\right\}_{m_{k}^{i-1} \in O S_{k}^{i-1}}\right)$ for $(\mathbb{E}[\mathbf{y}$ $\left.\left.\left(t_{k}^{i-1}\right) \mid \mathscr{F}_{k-1}^{i-1}\right], \mathbb{E}\left[\mathbf{p}\left(t_{k}^{i-1}\right) \mid \mathscr{F}_{k-1}^{i-1}\right]\right)$, we determine the following sub-optimal admissible set of $m_{k}^{i-1}$-size local conditional sample

$$
\left\{\mathscr{M}_{t_{k}^{i-1}}=\left\{m_{k}^{i-1} \in O S_{k}^{i-1}: \Xi_{m_{k}^{i-1}, t_{k}^{i-1}}<\varepsilon\right\}, \quad \text { for } i \in I\left(1, K^{*}\right)\right.
$$

Among these collected values, the value that gives the minimum $\Xi_{m_{k}^{i-1}, t_{k}^{i-1}}$ for $k \in\left[0, N_{i-1}\right]$ are recorded as $\widehat{m}_{k}^{i-1}$. If more than one value exist, then the largest of such $m_{k}^{i-1}$ 's is recorded as $\widehat{m}_{k}^{\mathrm{i}-1}$. If condition (86) is not met at time $t_{k}^{i-1}$, the value of $m_{k}^{i-1}$ where the minimum $\min _{m_{k}^{i-1}} \Xi_{m_{k}^{i-1}, t_{k}^{i-1}}$ is attained is recorded as $\widehat{m}_{k}^{i-1}$. The $\varepsilon$ - level sub-optimal estimates of the parameters $\left\{\widehat{u}_{j}^{i-1}\left(m_{k}^{i-1}, t_{k}^{i-1}\right), \widehat{\kappa}_{j, l}^{i-1}\left(m_{k}^{i-1}, t_{k}^{i-1}\right), \widehat{\beta}_{j}^{i-1}\left(m_{k}^{i-1}\right.\right.$, $\left.\left.t_{k}^{i-1}\right), \widehat{\delta}_{j, l}^{i-1}\left(m_{k}^{i-1}, t_{k}^{i-1}\right), \widehat{\gamma}_{j, l}^{i-1}\left(m_{k}^{i-1}, t_{k}^{i-1}\right), \widehat{\sigma}_{j, l}^{i-1}\left(m_{k}^{i-1}, t_{k}^{i-1}\right)\right\} \quad$ are recorded as $\left\{u_{j}^{i-1}\left(\widehat{m}_{k, y_{j}}^{i-1}, k\right), \kappa_{j, l}^{i-1}\left(\widehat{m}_{k, y_{j}}^{i-1}, k\right), \beta_{j}^{i-1}\left(\widehat{m}_{k, p_{j}}^{i-1}, k\right), \delta_{j, l}^{i-1}\right.$ $\left.\left(\widehat{m}_{k, y_{j}}^{i-1}, k\right), \gamma_{j, l}^{i-1}\left(\widehat{m}_{k, p_{j}}^{i-1}, k\right), \sigma_{j, l}^{i-1}\left(\widehat{m}_{k, p_{j}}^{i-1}, k\right)\right\}$. Finally, the simulated value $\mathbf{y}^{s}\left(m_{k}^{i-1}, t_{k}^{i-1}\right), \mathbf{p}^{s}\left(m_{k}^{i-1}, t_{k}^{i-1}\right)$ at time $t_{k}^{i-1}$ with $\hat{m}_{k}^{i-1}$ is now recorded as the best estimate for $\mathbb{E}\left[\mathbf{y}^{i-1}\left(t_{k}^{i-1}\right) \mid \mathscr{F}_{k-1}^{i-1}\right]$ and $\mathbb{E}\left[\mathbf{p}^{i-1}\left(t_{k}^{i-1}\right) \mid \mathscr{F}_{k-1}^{i-1}\right]$. The value $\mathbf{y}^{s}\left(\widehat{m}_{k}^{i-1}, k\right), \mathbf{p}^{s}\left(\widehat{m}_{k}^{i-1}, k\right)$ is called the $\varepsilon$ - sub-optimal simulated value of $\mathbf{y}^{s}\left(m_{k}^{i-1}, t_{k}^{i-1}\right)$ and $\mathbf{p}^{s}\left(m_{k}^{i-1}, t_{k}^{i-1}\right)$ of $\mathbb{E}\left[\mathbf{y}^{i-1}\left(t_{k}^{i-1}\right) \mid \mathscr{F}_{k-1}^{i-1}\right]$ and $\mathbb{E}\left[\mathbf{p}^{i-1}\left(t_{k}^{i-1}\right) \mid\right.$ $\left.\mathscr{F}_{k-1}^{i-1}\right]$ at $t_{k}^{i-1}$.

7.5. Illustration: Application of Conceptual Computational Algorithm to Energy Commodity Data Set. In this subsection, we apply the above conceptual computational algorithm to study the relationship between three energy commodities by setting $n=3$ in (56). The three energy commodities are daily Henry Hub Natural gas data set, daily crude oil data set, and daily coal data set for the period of 05/04/2009$01 / 03 / 2014,[35-37]$. Thus, for each pair $\left(y_{1}, p_{1}\right),\left(y_{2}, p_{2}\right)$, and $\left(y_{3}, p_{3}\right)$, the drift and diffusion coefficient function of the stochastic dynamic equation governing $\left(y_{j}, p_{j}\right)$, for $j \in I$ ( $1,3)$ has 4 and 3 parameters each to be estimated, respectively. Thus, there are 42 parameters to be estimated in total. Using $\Delta t=1, \varepsilon=0.001$, for each $j \in I(1,3)$, the $\varepsilon$ - level suboptimal estimates of parameters $u_{j}^{i-1}\left(\widehat{m}_{k}^{i-1}, k\right), \beta_{j}^{i-1}\left(\widehat{m}_{k}^{i-1}, k\right)$, $\kappa_{j, l}^{i-1}\left(\widehat{m}_{k}^{i-1}, k\right), \gamma_{j, l}^{i-1}\left(\widehat{m}_{k}^{i-1}, k\right), \quad \delta_{j, l}^{i-1}\left(\widehat{m}_{k}^{\mathrm{i}-1}, k\right), \quad \sigma_{j, l}^{i-1}\left(\widehat{m}_{k}^{i-1}, k\right), l \in$ $I(1,3)$, at each real data times are exhibited below.

7.5.1. Illustration: Relationship between Natural Gas, Crude Oil, and Coal: Without Incorporating Jump Process. In this subsubsection, we analyze the relationship between natural gas, crude oil, and coal without the jump process. For $j, l \in I$ $(1,3)$, the stochastic dynamic system governing the three energy commodities is described in (59) of Remark 12. Here, $\left(y_{1}, p_{1}\right)$ denotes the mean spot and the spot price process of 
natural gas, $\left(y_{2}, p_{2}\right)$ denotes the mean spot and the spot price process of crude oil, and $\left(y_{3}, p_{3}\right)$ denotes the mean spot and the spot price process of coal.

Using the discretized scheme (82), we apply the above conceptual computational algorithm for the real-time data sets namely daily Henry Hub natural gas data set, daily crude oil data set, and daily coal data set for the period of 05/04/2009-01/03/2014, [35-37]. Using $\Delta t=1, \varepsilon=0.001$, $r=10$, and $d=2$, the $\varepsilon$-level suboptimal estimates of the parameters at each real data times are described below for each commodity data sets.

The parameters corresponding to the natural gas data set are $u_{1}\left(\widehat{m}_{k}, k\right), \beta_{1}\left(\widehat{m}_{k}, k\right), \kappa_{1,1}\left(\widehat{m}_{k}, k\right), \kappa_{1,2}\left(\widehat{m}_{k}, k\right), \kappa_{1,3}\left(\widehat{m}_{k}, k\right)$, $\gamma_{1,1}\left(\widehat{m}_{k}, k\right), \gamma_{1,2}\left(\widehat{m}_{k}, k\right), \gamma_{1,3}\left(\widehat{m}_{k}, k\right), \delta_{1,1}\left(\widehat{m}_{k}, k\right), \delta_{1,2}\left(\widehat{m}_{k}, k\right)$, $\delta_{1,3}\left(\widehat{m}_{k}, k\right), \quad \sigma_{1,1}\left(\widehat{m}_{k}, k\right), \quad \sigma_{1,2}\left(\widehat{m}_{k}, k\right)$, and $\sigma_{1,3}\left(\widehat{m}_{k}, k\right)$. The parameters corresponding to the crude oil data set are $u_{2}$ $\left(\widehat{m}_{k}, k\right), \beta_{2}\left(\widehat{m}_{k}, k\right), \kappa_{2,1}\left(\widehat{m}_{k}, k\right), \kappa_{2,2}\left(\widehat{m}_{k}, k\right), \kappa_{2,3}\left(\widehat{m}_{k}, k\right), \gamma_{2,1}$ $\left(\widehat{m}_{k}, k\right), \gamma_{2,2}\left(\widehat{m}_{k}, k\right), \gamma_{2,3}\left(\widehat{m}_{k}, k\right), \delta_{2,1}\left(\widehat{m}_{k}, k\right), \delta_{2,2}\left(\widehat{m}_{k}, k\right), \delta_{2,3}$ $\left(\widehat{m}_{k}, k\right), \quad \sigma_{2,1}\left(\widehat{m}_{k}, k\right), \quad \sigma_{2,2}\left(\widehat{m}_{k}, k\right), \quad$ and $\sigma_{2,3}\left(\widehat{m}_{k}, k\right)$. The parameters corresponding to coal data set are $u_{3}\left(\widehat{m}_{k}, k\right), \beta_{3}$ $\left(\widehat{m}_{k, p_{3}}, k\right), \quad \kappa_{3,1}\left(\widehat{m}_{k}, k\right), \quad \kappa_{3,2}\left(\widehat{m}_{k}, k\right), \quad \kappa_{3,3}\left(\widehat{m}_{k}, k\right), \quad \gamma_{3,1}\left(\widehat{m}_{k}, k\right)$, $\gamma_{3,2}\left(\widehat{m}_{k}, k\right), \gamma_{3,3}\left(\widehat{m}_{k}, k\right), \delta_{3,1}\left(\widehat{m}_{k}, k\right), \delta_{3,2}\left(\widehat{m}_{k}, k\right), \delta_{3,3}\left(\widehat{m}_{k}, k\right)$, $\sigma_{3,1}\left(\widehat{m}_{k}, k\right), \sigma_{3,2}\left(\widehat{m}_{k}, k\right)$, and $\sigma_{3,3}\left(\widehat{m}_{k}, k\right)$.

The following table gives the drift coefficient's parameter estimates $u_{1}\left(\widehat{m}_{k}, k\right), \kappa_{1,1}\left(\widehat{m}_{k}, k\right), \kappa_{1,2}\left(\widehat{m}_{k}, k\right), \kappa_{1,3}\left(\widehat{m}_{k}, k\right), u_{2}$ $\left(\widehat{m}_{k}, k\right), \kappa_{2,1}\left(\widehat{m}_{k}, k\right), \kappa_{2,2}\left(\widehat{m}_{k}, k\right), \kappa_{2,3}\left(\widehat{m}_{k}, k\right), u_{3}\left(\widehat{m}_{k}, k\right), \kappa_{3,1}$ $\left(\widehat{m}_{k}, k\right), \kappa_{3,2}\left(\widehat{m}_{k}, k\right)$, and $\kappa_{3,3}\left(\widehat{m}_{k}, k\right)$ for the decoupled dynamical system for $y$ in the case where jump is not incorporated into the dynamical system.

Table 1 shows the estimates of the $\varepsilon$ suboptimal size $\widehat{m}_{k}$, $j \in I(1,3)$, the parameters $u_{1}\left(\widehat{m}_{k}, k\right), \kappa_{1,1}\left(\widehat{m}_{k}, k\right), \kappa_{1,2}\left(\widehat{m}_{k}, k\right)$, $\kappa_{1,3}\left(\widehat{m}_{k}, k\right), u_{2}\left(\widehat{m}_{k}, k\right), \kappa_{2,1}\left(\widehat{m}_{k}, k\right), \kappa_{2,2}\left(\widehat{m}_{k}, k\right), \kappa_{2,3}\left(\widehat{m}_{k}, k\right), u_{3}$ $\left(\widehat{m}_{k}, k\right), \kappa_{3,1}\left(\widehat{m}_{k}, k\right), \kappa_{3,2}\left(\widehat{m}_{k}, k\right)$, and $\kappa_{3,3}\left(\widehat{m}_{k}, k\right)$ for each of the energy commodity data sets. Moreover, $d \leq r$ and the initial real data time is $t_{r}=t_{10}$.

Figure 1 shows the drift coefficient's parameter estimates $u_{1}\left(\widehat{m}_{k}, k\right), \quad u_{2}\left(\widehat{m}_{k}, k\right)$, and $u_{3}\left(\widehat{m}_{k}, k\right)$ for the decoupled dynamical system for $\mathbf{y}$ in the case where jump is not incorporated into the dynamical system.

Figures 1 (a)-1(c) are the graphs of $u_{1}\left(\widehat{m}_{k}, k\right), u_{2}\left(\widehat{m}_{k}, k\right)$, and $u_{3}\left(\widehat{m}_{k}, k\right)$ against time $t_{k}$ for the daily Henry Hub natural gas price [36], daily crude oil price [37], and daily coal price [35] data set, respectively. By plotting the real data sets, it is easily seen that the graphs of $u_{1}\left(\widehat{m}_{k}, k\right), u_{2}\left(\widehat{m}_{k}, k\right)$, and $u_{3}\left(\widehat{m}_{k}, k\right)$ are similar to the graph of the Henry Hub natural gas, crude oil, and coal data set, respectively. We expect this to happen because $u_{j}, j \in I(1,3)$, are the equilibrium spot price processes of (5). The sample means of the real spot price data sets for natural gas, crude oil, and coal are given by $3.7218,88.5620$, and 16.5543 , respectively. It can be seen from Figures 1 (a)-1(c) that the graphs of $u_{1}\left(\widehat{m}_{k}, k\right), u_{2}$ $\left(\widehat{m}_{k}, k\right)$, and $u_{3}\left(\widehat{m}_{k}, k\right)$ move around the mean values $3.7218,88.5620$, and 16.5543 , respectively. This analysis shows that the parameters $u_{j}, j \in I(1,3)$, are statistic processes for the respective mean of the data sets at time $t_{k}$.

The graph of the interaction parameters $\kappa_{1,1}\left(\widehat{m}_{k}, k\right), \kappa_{1,2}$ $\left(\widehat{m}_{k}, k\right), \kappa_{1,3}\left(\widehat{m}_{k}, k\right), \kappa_{2,1}\left(\widehat{m}_{k}, k\right), \kappa_{2,2}\left(\widehat{m}_{k}, k\right), \kappa_{2,3}\left(\widehat{m}_{k}, k\right), \kappa_{3,1}$ $\left(\widehat{m}_{k}, k\right), \kappa_{3,2}\left(\widehat{m}_{k}, k\right)$, and $\kappa_{3,3}\left(\widehat{m}_{k}, k\right)$ for the decoupled dynamical system for $y$ (with no jump incorporated into the dynamical system) are given in Figure 2 below.

Figures 2(a)-2(i) show the graph of the $\varepsilon$ suboptimal interaction coefficient parameters $\kappa_{1,1}\left(\widehat{m}_{k}, k\right), \kappa_{1,2}\left(\widehat{m}_{k}, k\right)$, $\kappa_{1,3}\left(\widehat{m}_{k}, k\right), \kappa_{2,1}\left(\widehat{m}_{k}, k\right), \kappa_{2,2}\left(\widehat{m}_{k}, k\right), \kappa_{2,3}\left(\widehat{m}_{k}, k\right), \kappa_{3,1}\left(\widehat{m}_{k}, k\right)$, $\kappa_{3,2}\left(\widehat{m}_{k}, k\right)$, and $\kappa_{3,3}\left(\widehat{m}_{k}, k\right)$. The interaction coefficients $\kappa_{j, l}$, $j \neq l$ are negligible, because each estimate is $<<10^{-15}$. Thus, this shows that the model describing the mean spot price, $y_{j}$, is mainly characterized by the market potential $\kappa_{j, j}\left(u_{j}-\right.$ $\left.y_{j}\right) y_{j}, j \in I(1, n)$.

Table 2 below shows the estimates of the diffusion coefficient's parameters $\delta_{1,1}\left(\widehat{m}_{k}, k\right), \delta_{1,2}\left(\widehat{m}_{k}, k\right), \delta_{1,3}\left(\widehat{m}_{k}, k\right), \delta_{2,1}$ $\left(\widehat{m}_{k}, k\right), \delta_{2,2}\left(\widehat{m}_{k}, k\right), \delta_{2,3}\left(\widehat{m}_{k}, k\right), \delta_{3,1}\left(\widehat{m}_{k}, k\right), \delta_{3,2}\left(\widehat{m}_{k}, k\right)$, and $\delta_{3,3}\left(\widehat{m}_{k}, k\right)$ for the decoupled dynamical system for $\mathbf{y}$ in the case where jump is not incorporated into the dynamical system.

The graph of the diffusion coefficient's parameter for the decoupled dynamical system for $y$ without jump incorporated into the dynamical system are given in Figure 3 below.

Figures 3(a)-3(i) show the graphs of the $\varepsilon$ suboptimal interaction measure of fluctuation coefficient parameters $\delta_{1,1}\left(\widehat{m}_{k}, k\right), \delta_{1,2}\left(\widehat{m}_{k}, k\right), \delta_{1,3}\left(\widehat{m}_{k}, k\right), \delta_{2,1}\left(\widehat{m}_{k}, k\right), \delta_{2,2}\left(\widehat{m}_{k}, k\right)$, $\delta_{2,3}\left(\widehat{m}_{k}, k\right), \delta_{3,1}\left(\widehat{m}_{k}, k\right), \delta_{3,2}\left(\widehat{m}_{k}, k\right)$, and $\delta_{3,3}\left(\widehat{m}_{k}, k\right)$.

Table 3 gives the drift coefficient's parameter estimates $\beta_{1}\left(\widehat{m}_{k}, k\right), \gamma_{1,1}\left(\widehat{m}_{k}, k\right), \gamma_{1,2}\left(\widehat{m}_{k}, k\right), \gamma_{1,3}\left(\widehat{m}_{k}, k\right), \beta_{2}\left(\widehat{m}_{k}, k\right), \gamma_{2,1}$ $\left(\widehat{m}_{k}, k\right), \gamma_{2,2}\left(\widehat{m}_{k}, k\right), \gamma_{2,3}\left(\widehat{m}_{k}, k\right), \beta_{3}\left(\widehat{m}_{k}, k\right), \gamma_{3,1}\left(\widehat{m}_{k}, k\right), \gamma_{3,2}$ $\left(\widehat{m}_{k}, k\right)$, and $\gamma_{3,3}\left(\widehat{m}_{k}, k\right)$ for the dynamical system for $\mathbf{p}$ without jump.

Table 3 shows the estimates of the $\varepsilon$ suboptimal size $\widehat{m}_{k}$, $j \in I(1,3)$ and the parameters $\beta_{1}\left(\widehat{m}_{k}, k\right), \gamma_{1,1}\left(\widehat{m}_{k}, k\right), \gamma_{1,2}\left(\widehat{m}_{k}\right.$ , k), $\gamma_{1,3}\left(\widehat{m}_{k}, k\right), \beta_{2}\left(\widehat{m}_{k}, k\right), \gamma_{2,1}\left(\widehat{m}_{k}, k\right), \gamma_{2,2}\left(\widehat{m}_{k}, k\right), \gamma_{2,3}\left(\widehat{m}_{k}, k\right.$ ), $\beta_{3}\left(\widehat{m}_{k}, k\right), \gamma_{3,1}\left(\widehat{m}_{k}, k\right), \gamma_{3,2}\left(\widehat{m}_{k}, k\right)$, and $\gamma_{3,3}\left(\widehat{m}_{k}, k\right)$, for each of the energy commodity data sets. Moreover, $d \leq r$, and the initial real data time is $t_{r}=t_{10}$.

In the following, we give the graph of the drift coefficient's parameters with estimates in Table 3 in Figure 4 below.

Figures 4(a)-4(i) show the graphs of the $\varepsilon$ suboptimal interaction coefficient parameters $\gamma_{1,1}\left(\widehat{m}_{k}, k\right), \gamma_{1,2}\left(\widehat{m}_{k}, k\right)$, $\gamma_{1,3}\left(\widehat{m}_{k}, k\right), \gamma_{2,1}\left(\widehat{m}_{k}, k\right), \gamma_{2,2}\left(\widehat{m}_{k}, k\right), \gamma_{2,3}\left(\widehat{m}_{k}, k\right), \gamma_{3,1}\left(\widehat{m}_{k}, k\right)$, $\gamma_{3,2}\left(\widehat{m}_{k}, k\right)$, and $\gamma_{3,3}\left(\widehat{m}_{k}, k\right)$. According to (58), the estimate $\gamma_{j, l}\left(\widehat{m}_{k}, k\right), j \neq l$, is positive if commodity $p_{l}$ is cooperating with commodity $p_{j}$, and negative if commodity $p_{l}$ is competing with commodity $p_{j}$. There is no interaction between the two commodities if $\gamma_{j, l}\left(\widehat{m}_{k}, k\right)=0$. It is apparent from the graph of $\gamma_{1,3}\left(\widehat{m}_{k}, k\right)$ that coal is competing with natural gas because the estimates of $\gamma_{1,3}\left(\widehat{m}_{k}, k\right)$ are mostly negative. Also, it is apparent that natural gas and crude oil are either cooperating or competing, depending on the time period.

Figures $5(\mathrm{a})-5(\mathrm{c})$ are the graphs of $\beta_{1}\left(\widehat{m}_{k}, k\right), \beta_{2}\left(\widehat{m}_{k}, k\right)$, and $\beta_{3}\left(\widehat{m}_{k}, k\right)$ against time $t_{k}$ for the daily Henry Hub natural gas price data set [36], daily crude oil price data set [37], and daily coal price data set, respectively. 
TABLE 11: Estimates $\sigma_{1,1}\left(\widehat{m}_{k}, k\right), \sigma_{1,2}\left(\widehat{m}_{k}, k\right), \sigma_{1,3}\left(\widehat{m}_{k}, k\right), \sigma_{2,1}\left(\widehat{m}_{k}, k\right), \sigma_{2,2}\left(\widehat{m}_{k}, k\right), \sigma_{2,3}\left(\widehat{m}_{k}, k\right), \sigma_{3,1}\left(\widehat{m}_{k}, k\right), \sigma_{3,2}\left(\widehat{m}_{k}, k\right)$, and $\sigma_{3,3}\left(\widehat{m}_{k}, k\right)$.

\begin{tabular}{|c|c|c|c|c|c|c|c|c|c|}
\hline$t_{k}$ & $\sigma_{1,1}\left(\widehat{m}_{k}, k\right)$ & $\begin{array}{c}\text { Natural gas } \\
\sigma_{1,2}\left(\widehat{m}_{k}, k\right)\end{array}$ & $\sigma_{1,3}\left(\widehat{m}_{k}, k\right)$ & $\sigma_{2,1}\left(\widehat{m}_{k}, k\right)$ & $\begin{array}{c}\text { Crude oil } \\
\sigma_{2,2}\left(\widehat{m}_{k}, k\right)\end{array}$ & $\sigma_{2,3}\left(\widehat{m}_{k}, k\right)$ & $\sigma_{3,1}\left(\widehat{m}_{k}, k\right)$ & $\begin{array}{c}\text { Coal } \\
\sigma_{3,2}\left(\widehat{m}_{k}, k\right)\end{array}$ & $\sigma_{3,3}\left(\widehat{m}_{k}, k\right)$ \\
\hline 11 & 0 & 0 & 0 & 0 & 0 & 0 & 0 & 0.1303 & 0 \\
\hline 12 & 0.0485 & 0.0004 & 0.0032 & 0.2734 & 0.0166 & 0 & 0.0513 & 0 & 0.0000 \\
\hline 13 & 0.7333 & 0 & 0 & 0.9445 & 0 & 0 & 0.2489 & 0 & 0 \\
\hline 14 & 0.2120 & 0.1386 & 0.0133 & 0.3877 & 0.4773 & 0.1195 & 0.1365 & 0.0665 & 0.0086 \\
\hline 15 & 0.4246 & 0.1318 & 0.0021 & 0.03341 & 0.4894 & 0.1211 & 0.0112 & 0.6107 & 0.0696 \\
\hline 16 & 0.5538 & 0.0778 & 0.1501 & 0.07751 & 0.2524 & 0.0811 & 0.0651 & 0.4251 & 0.0635 \\
\hline 17 & 0.3907 & 0.0469 & 0.2230 & 0.08746 & 0.1848 & 0.2463 & 0 & 0.4458 & 0.0478 \\
\hline 18 & 0.3523 & 0.0180 & 0.2178 & 0.04291 & 0.1877 & 0.1602 & 0.5681 & 0.0592 & 0.0115 \\
\hline 19 & 0.5116 & 0.0619 & 0.2221 & 0.03266 & 0.2673 & 0.2465 & 0.4999 & 0.0569 & 0.0127 \\
\hline 20 & 0.6431 & 0.0536 & 0.1866 & 0.0939 & 0.1700 & 0.0781 & 0.3789 & 0.3174 & 0.0046 \\
\hline$\ldots$ & $\ldots$ & $\ldots$ & $\ldots$ & $\ldots$ & $\ldots$ & $\ldots$ & $\ldots$ & $\ldots$ & $\ldots$ \\
\hline 495 & 0 & 0.0036 & 0.0406 & 0.2286 & 0.0600 & 0.0172 & 0.0110 & 0.9387 & 0.0182 \\
\hline 496 & 0.1588 & 0.0035 & 0.0107 & 0.08183 & 0.3163 & 0.0102 & 0 & 0 & 0.0016 \\
\hline 497 & 0.1551 & 0.0009 & 0.0065 & 0.07869 & 0.1453 & 0.4821 & 0.7777 & 0 & 0.0033 \\
\hline 498 & 0.1576 & 0.0011 & 0.0073 & 0.0120 & 0.1679 & 0.3786 & 0.5334 & 0 & 0.0060 \\
\hline 499 & 0.1197 & 0.0006 & 0.0059 & 0.0721 & 0.2391 & 0.0172 & 0.4405 & 0.1432 & 0.0097 \\
\hline 500 & 0.3600 & 0.0001 & 0.0049 & 0.0273 & 0.3960 & 0.0079 & 0.6331 & 0.1410 & 0.0093 \\
\hline 0.5010 & 0.0514 & 0.0033 & 0.0049 & 0.0182 & 0.3499 & 0.0111 & 0.7690 & 0.1376 & 0.0089 \\
\hline 0.5020 & 0.2503 & 0.0034 & 0.0042 & 0.0222 & 0.1744 & 0.0132 & 0.6198 & 0.1274 & 0.0066 \\
\hline 0.5030 & 0.1195 & 0.0147 & 0.0165 & 0 & 0.4283 & 0.0060 & 0 & 0.1530 & 0.0049 \\
\hline 0.5040 & 0.0974 & 0.0144 & 0.0027 & 0 & 0.2241 & 0.0048 & 0.4778 & 0.0574 & 0.0043 \\
\hline 0.5050 & 0.1422 & 0.0060 & 0.0131 & 0.0085 & 0.2023 & 0.0054 & 0.5604 & 0.0669 & 0.0004 \\
\hline$\ldots$ & $\ldots$ & $\ldots$ & $\ldots$ & $\ldots$ & $\ldots$ & $\ldots$ & $\ldots$ & $\ldots$ & $\ldots$ \\
\hline 1102 & 0.1898 & 0.0016 & 0.0413 & 0.8313 & 0.0767 & 0.0381 & 0.6875 & 0 & 0.1451 \\
\hline 1103 & 0.2094 & 0.0015 & 0.0352 & 0.8262 & 0.0673 & 0.0451 & 0.7298 & 0.2808 & 0.0147 \\
\hline 1104 & 0.1711 & 0.0011 & 0.0040 & 0.6648 & 0.0915 & 0.0462 & 0.5563 & 0.1831 & 0.0105 \\
\hline 1105 & 0.1816 & 0.0012 & 0.0116 & 0.6658 & 0.1049 & 0.0371 & 0.6591 & 0.2874 & 0.0057 \\
\hline 1106 & 0.1191 & 0.0011 & 0.0116 & 0.6260 & 0.1155 & 0.0393 & 0 & 0.0196 & 0.0060 \\
\hline 1107 & 0.0417 & 0.0012 & 0.0041 & 0.4992 & 0.0781 & 0.0382 & 0.0271 & 0.2559 & 0.0065 \\
\hline 1108 & 0.1058 & 0.0033 & 0.0045 & 0.0019 & 0.0589 & 0.0421 & 0.6209 & 0.8289 & 0.0018 \\
\hline 1109 & 0.1740 & 0.0021 & 0 & 0.0305 & 0.0446 & 0.0316 & 0.8431 & 0.2366 & 0.4511 \\
\hline 1110 & 0.2912 & 0.0021 & 0.0163 & 0.0385 & 0.0342 & 0.0037 & 0.2910 & 0.0489 & 0.0257 \\
\hline
\end{tabular}

Table 4 gives the $\varepsilon$ suboptimal estimates of the parameters $\sigma_{1,1}\left(\widehat{m}_{k}, k\right), \sigma_{1,2}\left(\widehat{m}_{k}, k\right), \sigma_{1,3}\left(\widehat{m}_{k}, k\right), \sigma_{2,1}\left(\widehat{m}_{k}, k\right), \sigma_{2,2}\left(\widehat{m}_{k}\right.$ $, k), \sigma_{2,3}\left(\widehat{m}_{k}, k\right), \sigma_{3,1}\left(\widehat{m}_{k}, k\right), \sigma_{3,2}\left(\widehat{m}_{k}, k\right)$, and $\sigma_{3,3}\left(\widehat{m}_{k}, k\right)$ for each of the energy commodity data sets.

Figures 6(a)-6(i) are the graphs of $\sigma_{1,1}\left(\widehat{m}_{k}, k\right), \sigma_{1,2}\left(\widehat{m}_{k}, k\right)$, $\sigma_{1,3}\left(\widehat{m}_{k}, k\right), \sigma_{2,1}\left(\widehat{m}_{k}, k\right), \sigma_{2,2}\left(\widehat{m}_{k}, k\right), \sigma_{2,3}\left(\widehat{m}_{k}, k\right), \sigma_{3,1}\left(\widehat{m}_{k}, k\right)$, $\sigma_{3,2}\left(\widehat{m}_{k}, k\right)$, and $\sigma_{1,1}\left(\widehat{m}_{k}, k\right)$ against time $t_{k}$ for the daily Henry Hub natural gas price data set [36], daily crude oil price data set [37], and daily coal price data set, respectively.

Table 5 shows the real and simulated estimates for the spot price processes $p_{j}(t), j \in I(1, n)$.

Figure 7 below shows the graph of the real and simulated prices for natural gas, crude oil, and coal data set.

Figures $7(a)-7(c)$ show the graph of the real and simulated spot prices for the daily Henry Hub natural gas data set [36], daily crude oil data set [37], and daily coal data set [35], respectively. The red line represents the real data set $p\left(t_{k}\right)$, while the blue line represent the simulated data set $p^{s}\left(\widehat{m}_{k}, k\right)$. Here, we begin by using a starting delay of $r=10$. The simulation starts from $t_{r}=t_{10}$. It is clear that the graph fits well. To reduce magnitude of error, we increase the magnitude of time delay. We later compare this result with the case where jump is incorporated into the system.

7.5.2. Relationship between Natural Gas, Crude Oil, and Coal: With Jump Incorporated. In this subsubsection, we analyze the relationship between natural gas, crude oil, and coal with the jump process. Here, we apply the above conceptual computational algorithm described in this section for the realtime data sets, namely, daily Henry Hub natural gas data set, daily crude oil data set, and daily coal data set for the 


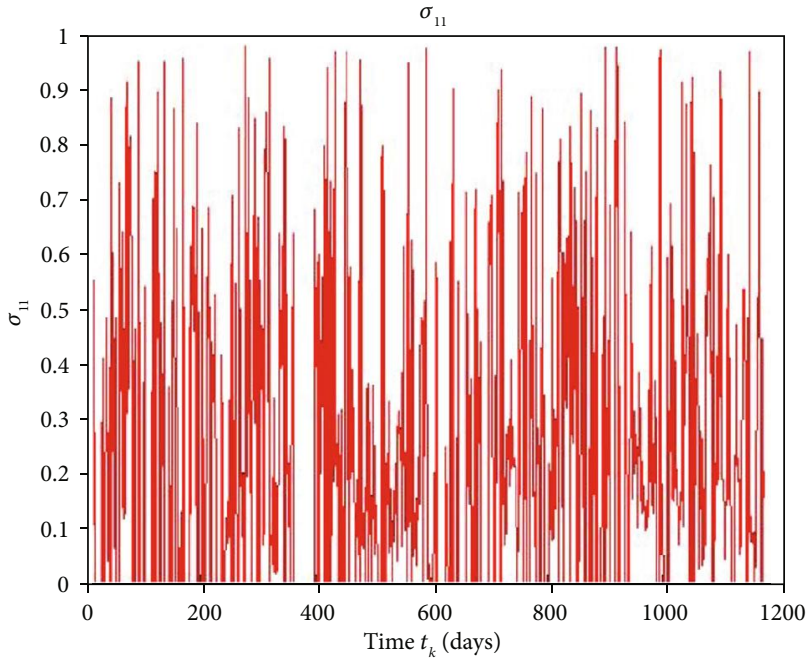

(a)

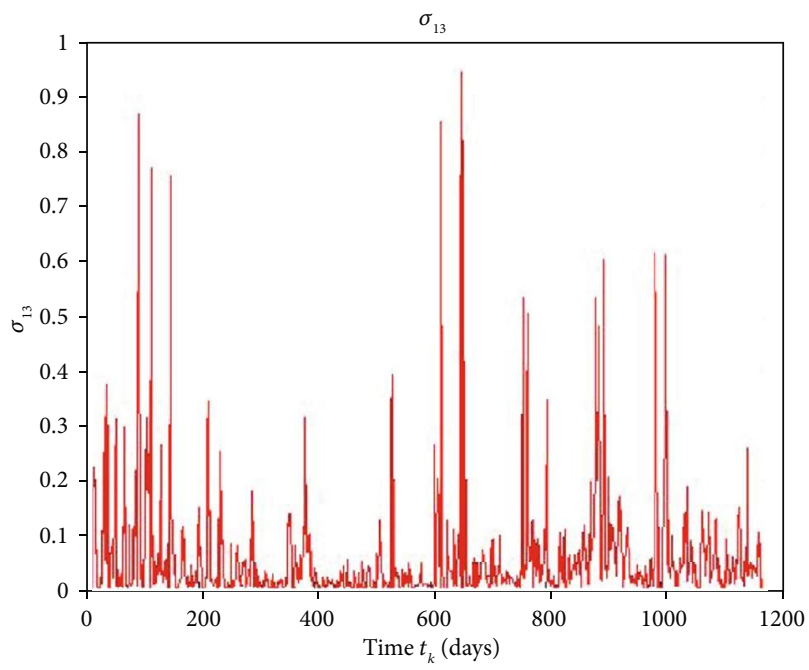

(c)

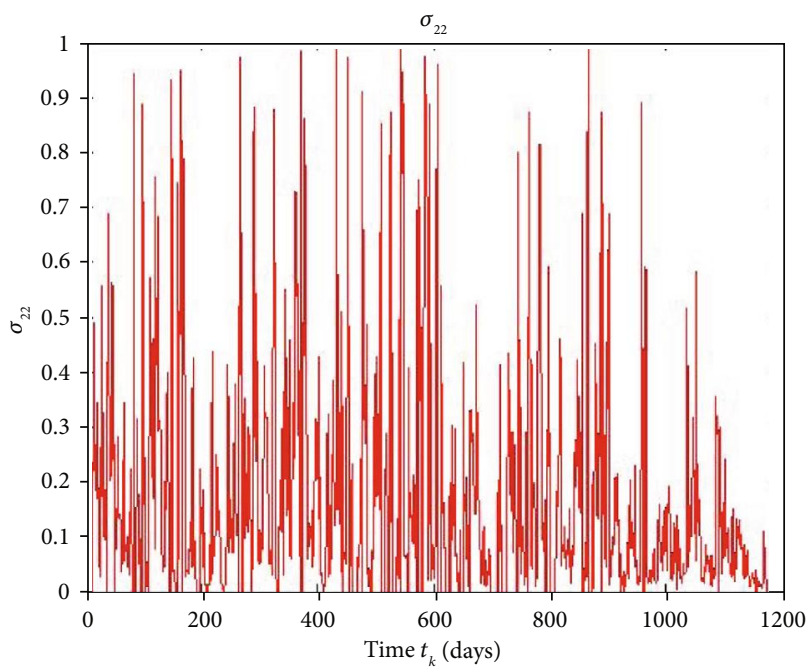

(e)

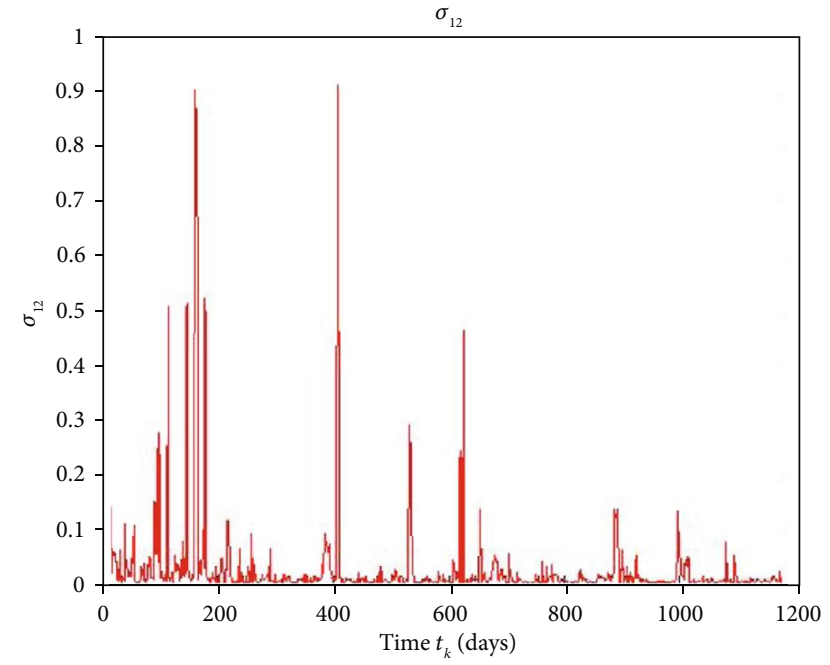

(b)

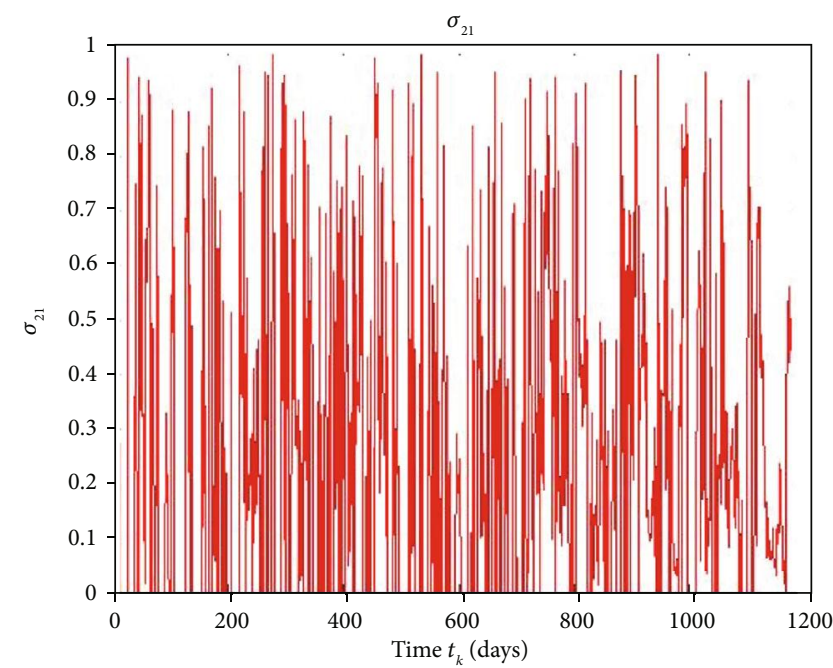

(d)

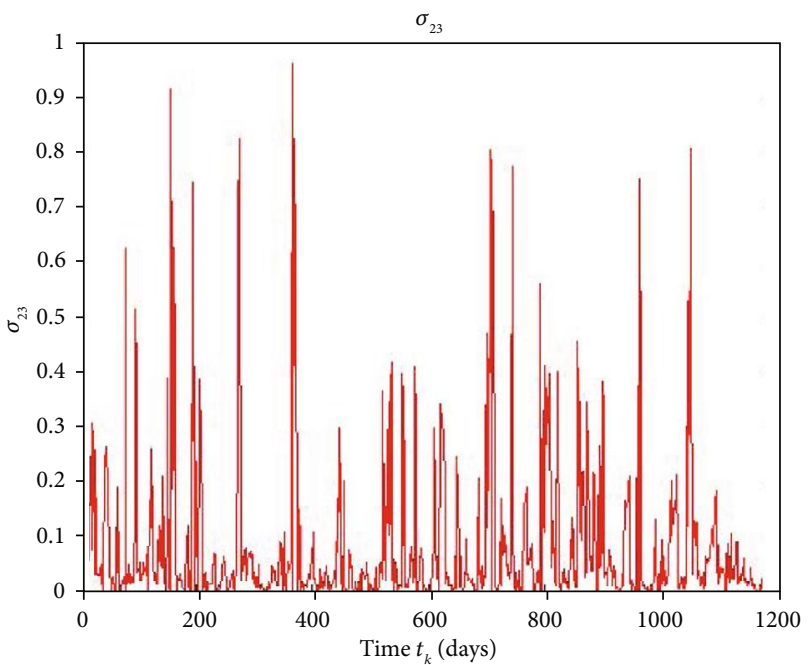

(f)

Figure 13: Continued. 


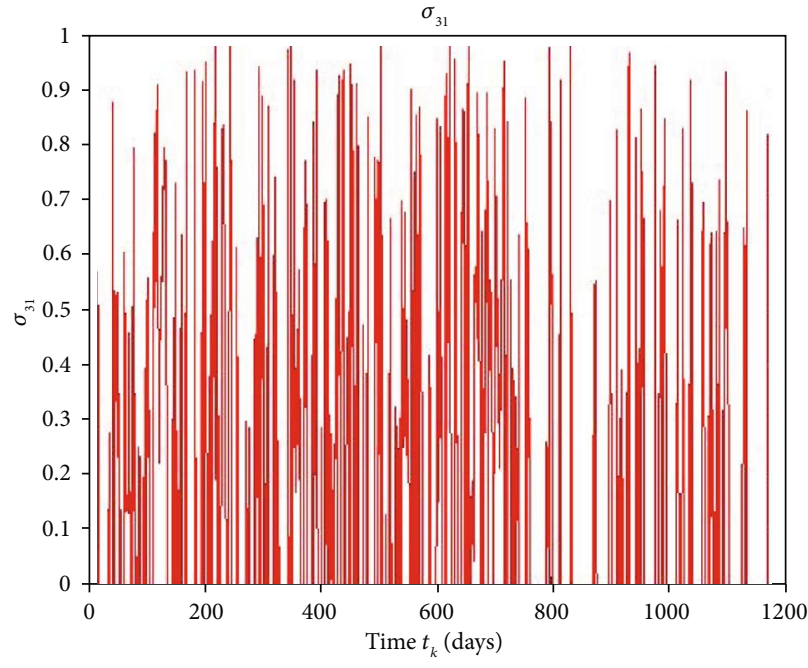

(g)

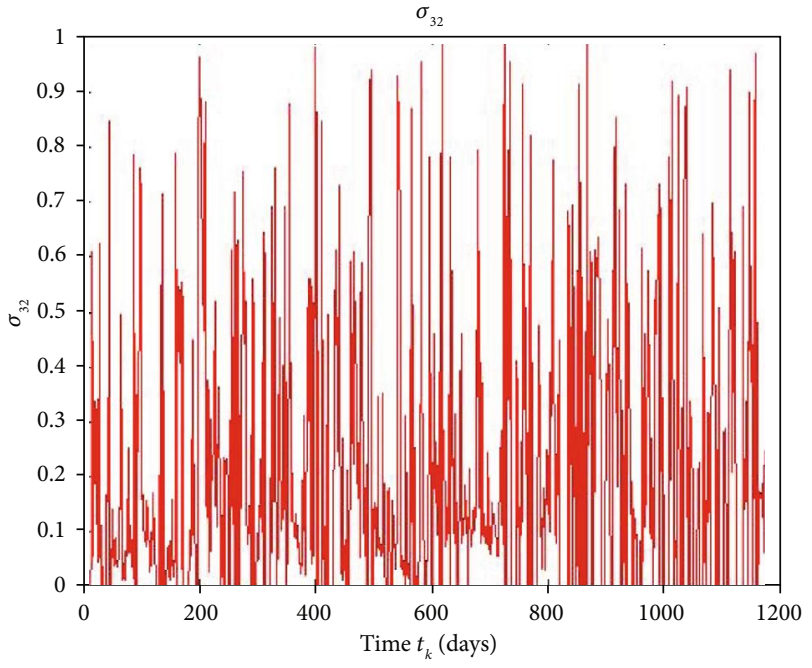

(h)

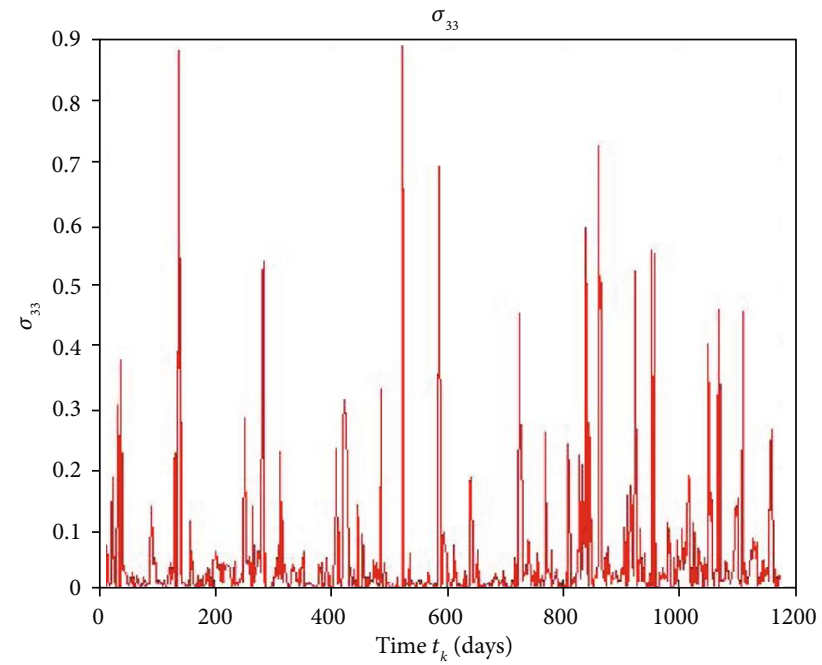

(i)

FigURE 13: The graph of $\sigma_{1,1}\left(\widehat{m}_{k}, k\right), \sigma_{1,2}\left(\widehat{m}_{k}, k\right), \sigma_{1,3}\left(\widehat{m}_{k}, k\right), \sigma_{2,1}\left(\widehat{m}_{k}, k\right), \sigma_{2,2}\left(\widehat{m}_{k}, k\right), \sigma_{2,3}\left(\widehat{m}_{k}, k\right), \sigma_{3,1}\left(\widehat{m}_{k}, k\right), \sigma_{3,2}\left(\widehat{m}_{k}, k\right)$, and $\sigma_{3,3}\left(\widehat{m}_{k}, k\right)$ for natural gas, crude oil, and coal, respectively.

period of 05/04/2009-01/03/2014, [35-37]. For $i \in I\left(1, K^{*}\right)$, $K \neq 0$, we use $\Delta t_{i-1}=1, \varepsilon=0.001, r_{i-1}=10$, and $d_{i-1}=2$. The $\varepsilon$-level suboptimal estimates of the parameters at each real data times are described below for each commodity data sets.

The parameters corresponding to the model governing natural gas price data set are $u_{1}^{i-1}\left(\widehat{m}_{k}, k\right), \beta_{1}^{i-1}\left(\widehat{m}_{k}^{i-1}, t_{k}^{i-1}\right)$, $\kappa_{1,1}^{i-1}\left(\widehat{m}_{k}^{i-1}, t_{k}^{i-1}\right), \kappa_{1,2}^{i-1}\left(\widehat{m}_{k}^{i-1}, t_{k}^{i-1}\right), \kappa_{1,3}^{i-1}\left(\widehat{m}_{k}^{i-1}, t_{k}^{i-1}\right), \gamma_{1,1}^{i-1}\left(\widehat{m}_{k}^{i-1}, t_{k}^{i-1}\right.$ )$, \gamma_{1,2}^{i-1}\left(\widehat{m}_{k}^{i-1}, t_{k}^{i-1}\right), \gamma_{1,3}^{i-1}\left(\widehat{m}_{k}^{i-1}, t_{k}^{i-1}\right), \delta_{1,1}^{i-1}\left(\widehat{m}_{k}^{i-1}, t_{k}^{i-1}\right), \delta_{1,2}^{i-1}\left(\widehat{m}_{k}^{i-1}\right.$, $\left.t_{k}^{i-1}\right), \quad \delta_{1,3}^{i-1}\left(\widehat{m}_{k}^{i-1}, t_{k}^{i-1}\right), \quad \sigma_{1,1}^{i-1}\left(\widehat{m}_{k}^{i-1}, t_{k}^{i-1}\right), \quad \sigma_{1,2}^{i-1}\left(\widehat{m}_{k}^{i-1}, t_{k}^{i-1}\right)$, and $\sigma_{1,3}^{i-1}\left(\widehat{m}_{k}^{i-1}, t_{k}^{i-1}\right)$. The parameters corresponding to the model governing crude oil price data set are $u_{2}^{i-1}\left(\widehat{m}_{k}^{i-1}, t_{k}^{i-1}\right), \beta_{2}^{i-1}$ $\left(\widehat{m}_{k}^{i-1}, t_{k}^{i-1}\right), \kappa_{2,1}^{i-1}\left(\widehat{m}_{k}^{i-1}, t_{k}^{i-1}\right), \kappa_{2,2}^{i-1}\left(\widehat{m}_{k}^{i-1}, t_{k}^{i-1}\right), \quad \kappa_{2,3}^{i-1}\left(\widehat{m}_{k}^{i-1}, t_{k}^{i-1}\right)$, $\gamma_{2,1}^{i-1}\left(\widehat{m}_{k}^{i-1}, t_{k}^{i-1}\right), \quad \gamma_{2,2}^{i-1}\left(\widehat{m}_{k}^{i-1}, t_{k}^{i-1}\right), \quad \gamma_{2,3}^{i-1}\left(\widehat{m}_{k}^{i-1}, t_{k}^{i-1}\right), \quad \delta_{2,1}^{i-1}\left(\widehat{m}_{k}^{i-1}\right.$, $\left.t_{k}^{i-1}\right), \quad \delta_{2,2}^{i-1}\left(\widehat{m}_{k}^{i-1}, t_{k}^{i-1}\right), \quad \delta_{2,3}^{i-1}\left(\widehat{m}_{k}^{i-1}, t_{k}^{i-1}\right), \quad \sigma_{2,1}^{i-1}\left(\widehat{m}_{k}^{i-1}, t_{k}^{i-1}\right), \quad \sigma_{2,2}^{i-1}$ $\left(\widehat{m}_{k}^{i-1}, t_{k}^{i-1}\right)$, and $\sigma_{2,3}^{i-1}\left(\widehat{m}_{k}^{i-1}, t_{k}^{i-1}\right)$, while the parameters corre- sponding to the model governing coal price data set are $u_{3}^{i-1}$ $\left(\widehat{m}_{k}^{i-1}, t_{k}^{i-1}\right), \beta_{3}^{i-1}\left(\widehat{m}_{k}^{i-1}, t_{k}^{i-1}\right), \kappa_{3,1}^{i-1}\left(\widehat{m}_{k}^{i-1}, t_{k}^{i-1}\right), \kappa_{3,2}^{i-1}\left(\widehat{m}_{k}^{i-1}, t_{k}^{i-1}\right)$, $\kappa_{3,3}^{i-1}\left(\widehat{m}_{k}^{i-1}, t_{k}^{i-1}\right), \quad \gamma_{3,1}^{i-1}\left(\widehat{m}_{k}^{i-1}, t_{k}^{i-1}\right), \quad \gamma_{3,2}^{i-1}\left(\widehat{m}_{k}^{i-1}, t_{k}^{i-1}\right), \quad \gamma_{3,3}^{i-1}\left(\widehat{m}_{k}^{i-1}\right.$, $\left.t_{k}^{i-1}\right), \quad \delta_{3,1}^{i-1}\left(\widehat{m}_{k}^{i-1}, t_{k}^{i-1}\right), \quad \delta_{3,2}^{i-1}\left(\widehat{m}_{k}^{i-1}, t_{k}^{i-1}\right), \quad \delta_{3,3}^{i-1}\left(\widehat{m}_{k}^{i-1}, t_{k}^{i-1}\right), \quad \sigma_{3,1}^{i-1}$ $\left(\widehat{m}_{k}^{i-1}, t_{k}^{i-1}\right), \sigma_{3,2}^{i-1}\left(\widehat{m}_{k}^{i-1}, t_{k}^{i-1}\right)$, and $\sigma_{3,3}^{i-1}\left(\widehat{m}_{k}^{i-1}, t_{k}^{i-1}\right)$.

For the sake of simplicity and in order to be able to compare our results in this subsection with the results in Subsection 7.5.1, for each $j, l \in I(1, n)$, we rewrite the parameters $u_{j}^{i-1}\left(\widehat{m}_{k}^{i-1}, t_{k}^{i-1}\right), \quad \beta_{j}^{i-1}\left(\widehat{m}_{k}^{i-1}, t_{k}^{i-1}\right), \quad \kappa_{j, l}^{i-1}\left(\widehat{m}_{k}^{i-1}, t_{k}^{i-1}\right), \quad \gamma_{j, l}^{i-1}\left(\widehat{m}_{k}^{i-1}\right.$, $\left.t_{k}^{i-1}\right), \delta_{j, l}^{i-1}\left(\widehat{m}_{k}^{i-1}, t_{k}^{i-1}\right)$, and $\sigma_{j, l}^{i-1}\left(\widehat{m}_{k}^{i-1}, t_{k}^{i-1}\right)$ after they have been estimated as $u_{j}\left(\widehat{m}_{k}, k\right), \beta_{j}\left(\widehat{m}_{k}, k\right), \kappa_{j, l}\left(\widehat{m}_{k}, k\right), \gamma_{j, l}\left(\widehat{m}_{k}, k\right), \delta_{j, l}$ $\left(\widehat{m}_{k}, k\right)$, and $\sigma_{j, l}\left(\widehat{m}_{k}, k\right)$.

We give some results for the jump times of the system $\left\{T_{i}\right\}_{i \in I\left(1, K^{*}\right)}$.

Table 6 shows some result for the jump times of the system $(\mathbf{y}, \mathbf{p})$. These results are derived by recording the times at 
TABLE 12: Real and simulated estimates (with jump) for natural gas, crude oil, and coal.

\begin{tabular}{|c|c|c|c|c|c|c|}
\hline \multirow[b]{2}{*}{$t_{k}$} & \multicolumn{2}{|c|}{ Natural gas } & \multicolumn{2}{|r|}{ Crude oil } & \multicolumn{2}{|r|}{ Coal } \\
\hline & Real & Simulated $p_{1}^{s}\left(\widehat{m}_{k}, k\right)$ & Real & Simulated $p_{2}^{s}\left(\widehat{m}_{k}, k\right)$ & Real & Simulated $p_{3}^{s}\left(\widehat{m}_{k}, k\right)$ \\
\hline 11 & 4.0200 & 4.0500 & 58.9900 & 58.5200 & 16.5900 & 16.6000 \\
\hline 12 & 3.9900 & 3.9600 & 59.5200 & 59.5099 & 17.4600 & 17.4635 \\
\hline 13 & 3.7500 & 3.6690 & 61.4500 & 60.3377 & 17.8900 & 17.8886 \\
\hline 14 & 3.7700 & 3.7341 & 60.4900 & 59.4191 & 17.5500 & 17.5188 \\
\hline 15 & 3.4100 & 3.3967 & 61.1500 & 60.1580 & 17.4100 & 17.4188 \\
\hline 16 & 3.3500 & 3.3947 & 62.4800 & 62.5028 & 16.7500 & 16.7789 \\
\hline 17 & 3.4900 & 3.3900 & 63.4100 & 63.5524 & 17.6600 & 17.5341 \\
\hline 18 & 3.5500 & 3.4957 & 65.0900 & 64.9224 & 17.5200 & 17.8130 \\
\hline 19 & 3.9200 & 3.8743 & 66.3100 & 66.8239 & 18.5000 & 18.8453 \\
\hline 20 & 3.8600 & 3.8241 & 68.5900 & 68.1206 & 19.0600 & 18.9453 \\
\hline$\cdots$ & $\cdots$ & $\cdots$ & $\ldots$ & $\ldots$ & $\ldots$ & $\ldots$ \\
\hline 494 & 4.2300 & 4.2295 & 106.7000 & 106.6374 & 33.2200 & 33.1852 \\
\hline 495 & 4.1900 & 4.2191 & 107.1800 & 106.9973 & 32.7600 & 32.6677 \\
\hline 496 & 4.3300 & 4.3414 & 110.8400 & 111.0084 & 33.6500 & 33.8070 \\
\hline 497 & 4.3300 & 4.3261 & 111.7200 & 111.7084 & 33.7100 & 33.7061 \\
\hline 498 & 4.3700 & 4.3863 & 111.6800 & 111.2084 & 34.7500 & 35.7057 \\
\hline 499 & 4.3200 & 4.2167 & 111.7200 & 111.6126 & 34.5400 & 33.5457 \\
\hline 500 & 4.3500 & 4.3577 & 112.3100 & 112.4358 & 34.0400 & 34.2862 \\
\hline 501 & 4.3800 & 4.3535 & 112.3800 & 112.5682 & 33.1000 & 33.1330 \\
\hline 502 & 4.5100 & 4.4389 & 113.3900 & 113.2925 & 33.6700 & 33.6216 \\
\hline 503 & 4.6000 & 4.6139 & 113.0300 & 113.3077 & 33.9400 & 33.9216 \\
\hline 504 & 4.6000 & 4.5964 & 110.6000 & 110.8350 & 33.8300 & 33.9216 \\
\hline 505 & 4.5900 & 4.5564 & 108.7900 & 108.7598 & 32.0200 & 31.9867 \\
\hline$\ldots$ & $\ldots$ & $\ldots$ & $\ldots$ & $\ldots$ & $\ldots$ & $\ldots$ \\
\hline 1102 & 3.7200 & 3.6616 & 108.2300 & 108.2354 & 4.7700 & 4.5482 \\
\hline 1103 & 3.7300 & 3.6477 & 106.2600 & 106.1451 & 5.0100 & 5.1120 \\
\hline 1104 & 3.6800 & 3.6748 & 104.7000 & 104.6723 & 4.9800 & 5.1180 \\
\hline 1105 & 3.6600 & 3.6861 & 103.6200 & 104.6723 & 4.7300 & 4.7893 \\
\hline 1106 & 3.5900 & 3.6436 & 103.2200 & 102.9765 & 4.6800 & 4.7074 \\
\hline 1107 & 3.5200 & 3.5213 & 102.6800 & 102.7652 & 4.6300 & 4.6419 \\
\hline 1108 & 3.4900 & 3.4564 & 103.1000 & 102.9765 & 4.7400 & 4.4016 \\
\hline 1109 & 3.5100 & 3.2596 & 102.8600 & 102.9652 & 4.3300 & 4.1826 \\
\hline 1110 & 3.4800 & 3.4604 & 102.3600 & 102.4345 & 4.1800 & 4.4606 \\
\hline
\end{tabular}

which an entry of a commodity differ by twice the standard deviation or more from the mean of that commodity. These times are now combined into a single array and sorted out in an increasing order.

We give the estimate of the jump coefficient matrices $\Pi^{i}$ and $\Theta^{i}$ defined in (34) in Table 7 below.

Table 8 gives the estimates $u_{1}\left(\widehat{m}_{k}, k\right), \kappa_{1,1}\left(\widehat{m}_{k}, k\right), \kappa_{1,2}$ $\left(\widehat{m}_{k}, k\right), \kappa_{1,3}\left(\widehat{m}_{k}, k\right), u_{2}\left(\widehat{m}_{k}, k\right), \kappa_{2,1}\left(\widehat{m}_{k}, k\right), \kappa_{2,2}\left(\widehat{m}_{k}, k\right), \kappa_{2,3}$ $\left(\widehat{m}_{k}, k\right), u_{3}\left(\widehat{m}_{k}, k\right), \kappa_{3,1}\left(\widehat{m}_{k}, k\right), \kappa_{3,2}\left(\widehat{m}_{k}, k\right)$, and $\kappa_{3,3}\left(\widehat{m}_{k}, k\right)$ for the decoupled dynamical system for $\mathbf{y}$ with jump.

Figure 8 shows the parameter estimates $u_{1}\left(\widehat{m}_{k}, k\right), u_{2}$ $\left(\widehat{m}_{k}, k\right)$, and $u_{3}\left(\widehat{m}_{k}, k\right)$ for the decoupled dynamical system for $\mathbf{y}$ with jump.

Figures $8(\mathrm{a})-8(\mathrm{c})$ are the graphs of $u_{1}\left(\widehat{m}_{k}, k\right), u_{2}\left(\widehat{m}_{k}, k\right)$, and $u_{3}\left(\widehat{m}_{k}, k\right)$ against time $t_{k}$ for the daily Henry Hub natural gas price data set [36], daily crude oil price data set [37], and daily coal price data set, respectively. By plotting the real data sets, it is easily seen that the graphs of $u_{1}\left(\widehat{m}_{k}, k\right), u_{2}\left(\widehat{m}_{k}, k\right)$, and $u_{3}\left(\widehat{m}_{k}, k\right)$ are similar to the graph of the Henry Hub natural gas, crude oil, and coal data set, respectively. We expect this to happen because $u_{j}, j \in I(1,3)$, are the equilibrium spot price processes of (5).

The graph of the interaction parameters $\kappa_{1,1}\left(\widehat{m}_{k}, k\right), \kappa_{1,2}$ $\left(\widehat{m}_{k}, k\right), \kappa_{1,3}\left(\widehat{m}_{k}, k\right), \kappa_{2,1}\left(\widehat{m}_{k}, k\right), \kappa_{2,2}\left(\widehat{m}_{k}, k\right), \kappa_{2,3}\left(\widehat{m}_{k}, k\right), \kappa_{3,1}$ $\left(\widehat{m}_{k}, k\right), \kappa_{3,2}\left(\widehat{m}_{k}, k\right)$, and $\kappa_{3,3}\left(\widehat{m}_{k}, k\right)$ for the decoupled dynamical system for $y$ with jump and estimates in Table 8 are given in Figure 9 below.

Figures 9(a)-9(i)) show the graph of the $\varepsilon$ suboptimal interaction coefficient parameters $\kappa_{1,1}\left(\widehat{m}_{k}, k\right), \kappa_{1,2}\left(\widehat{m}_{k}, k\right)$, $\kappa_{1,3}\left(\widehat{m}_{k}, k\right), \kappa_{2,1}\left(\widehat{m}_{k}, k\right), \kappa_{2,2}\left(\widehat{m}_{k}, k\right), \kappa_{2,3}\left(\widehat{m}_{k}, k\right), \kappa_{3,1}\left(\widehat{m}_{k}, k\right)$, $\kappa_{3,2}\left(\widehat{m}_{k}, k\right)$, and $\kappa_{3,3}\left(\widehat{m}_{k}, k\right)$. The interaction coefficients $\kappa_{j, l}$, $j \neq l$, are negligible, because each estimate is $<<10^{-15}$. Thus, 


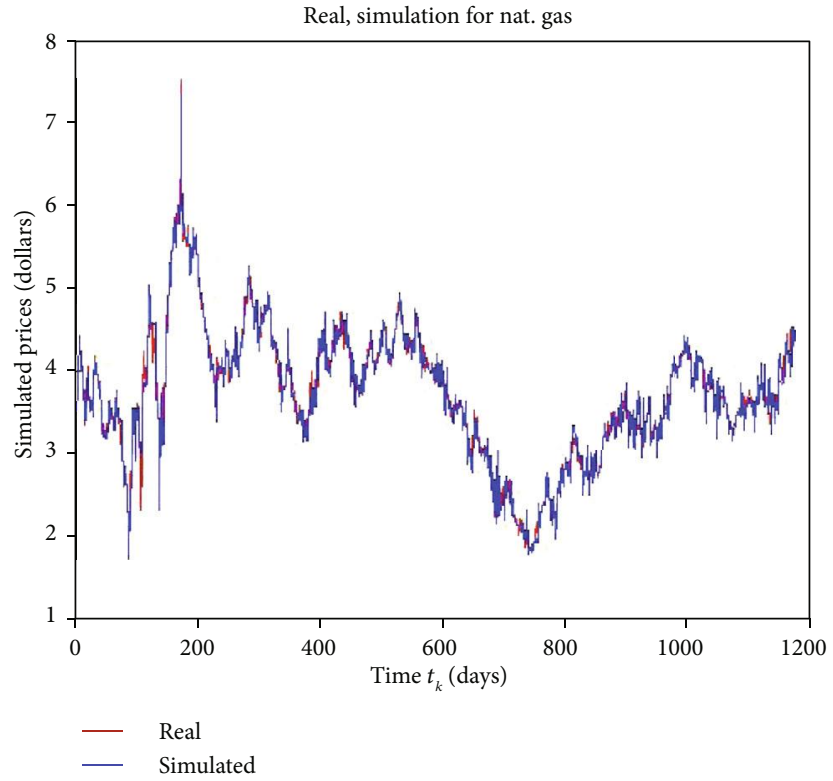

(a)

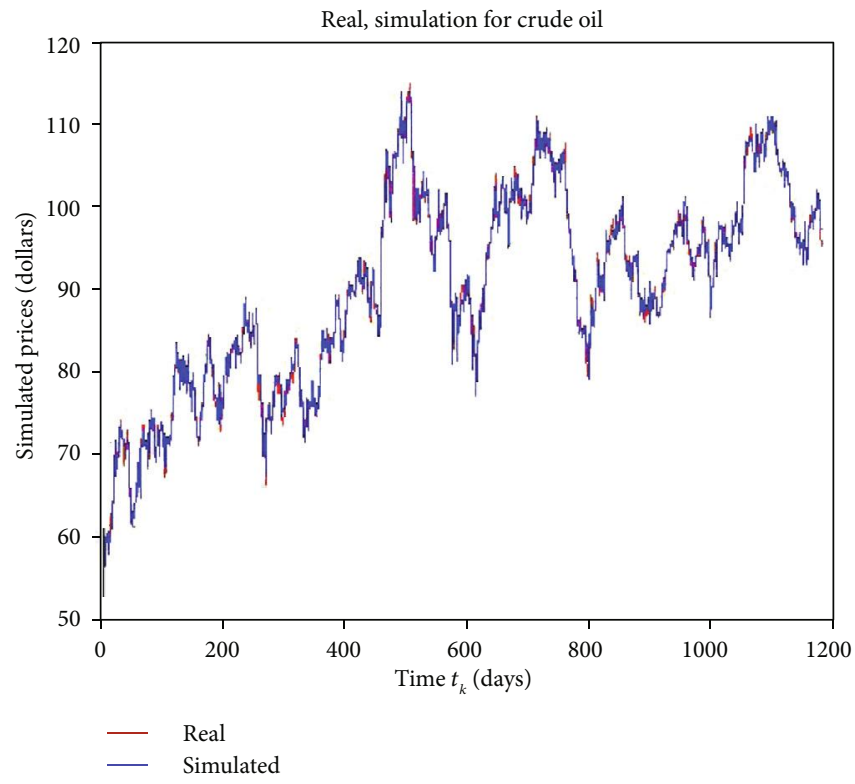

(b)

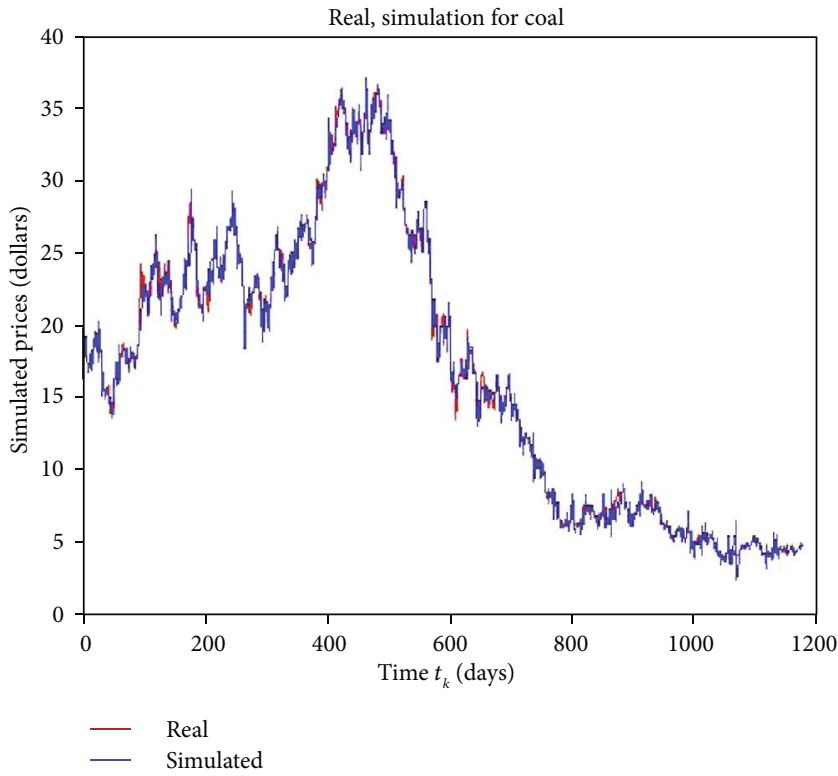

(c)

FIGURE 14: Real and simulated prices (with jump) for natural gas, crude oil, and coal.

this shows that the model describing the mean spot price, $y_{j}$, is mainly characterized by the market potential $\kappa_{j, j}^{i-1}\left(u_{j}^{i-1}-\right.$ $\left.y_{j}\right) y_{j}, j \in I(1, n), i \in I\left(1, K^{*}\right)$.

Table 9 below shows the estimates of the diffussion coefficient's parameters $\delta_{1,1}\left(\widehat{m}_{k}, k\right), \delta_{1,2}\left(\widehat{m}_{k}, k\right), \delta_{1,3}\left(\widehat{m}_{k}, k\right), \delta_{2,1}$ $\left(\widehat{m}_{k}, k\right), \delta_{2,2}\left(\widehat{m}_{k}, k\right), \delta_{2,3}\left(\widehat{m}_{k}, k\right), \delta_{3,1}\left(\widehat{m}_{k}, k\right), \delta_{3,2}\left(\widehat{m}_{k}, k\right)$, and $\delta_{3,3}\left(\widehat{m}_{k}, k\right)$ for the decoupled dynamical system for $\mathbf{y}$ with jump.

The graph of the diffusion coefficient's parameter for the decoupled dynamical system for $\mathbf{y}$ with jump are given in Figure 10 below.

Figures 10(a)-10(i) show the graph of the $\varepsilon$ - sub-optimal interaction measure of fluctuation coefficient parameters $\delta_{1,1}\left(\widehat{m}_{k}, k\right), \delta_{1,2}\left(\widehat{m}_{k}, k\right), \delta_{1,3}\left(\widehat{m}_{k}, k\right), \delta_{2,1}\left(\widehat{m}_{k}, k\right), \delta_{2,2}\left(\widehat{m}_{k}, k\right)$, $\delta_{2,3}\left(\widehat{m}_{k}, k\right), \delta_{3,1}\left(\widehat{m}_{k}, k\right), \delta_{3,2}\left(\widehat{m}_{k}, k\right)$, and $\delta_{3,3}\left(\widehat{m}_{k}, k\right)$.

Table 10 gives the drift coefficient's parameter estimates $\beta_{1}\left(\widehat{m}_{k}, k\right), \gamma_{1,1}\left(\widehat{\mathrm{m}}_{k}, k\right), \gamma_{1,2}\left(\widehat{m}_{k}, k\right), \gamma_{1,3}\left(\widehat{m}_{k}, k\right), \beta_{2}\left(\widehat{m}_{k}, k\right), \gamma_{2,1}$ $\left(\widehat{m}_{k}, k\right), \gamma_{2,2}\left(\widehat{m}_{k}, k\right), \gamma_{2,3}\left(\widehat{m}_{k}, k\right), \beta_{3}\left(\widehat{m}_{k}, k\right), \gamma_{3,1}\left(\widehat{m}_{k}, k\right), \gamma_{3,2}$ $\left(\widehat{m}_{k}, k\right)$, and $\gamma_{3,3}\left(\widehat{m}_{k}, k\right)$ for the dynamical system for $\mathbf{p}$ with jump.

Table 10 shows the estimates of the $\varepsilon$ suboptimal size $\widehat{m}_{k}$ and the parameters $\widehat{m}_{k}, \beta_{1}\left(\widehat{m}_{k}, k\right), \gamma_{1,1}\left(\widehat{m}_{k}, k\right), \gamma_{1,2}\left(\widehat{m}_{k}, k\right)$, $\gamma_{1,3}\left(\widehat{m}_{k}, k\right), \widehat{m}_{k}, \beta_{2}\left(\widehat{m}_{k}, k\right), \gamma_{2,1}\left(\widehat{m}_{k}, k\right), \gamma_{2,2}\left(\widehat{m}_{k}, k\right), \gamma_{2,3}\left(\widehat{m}_{k}\right.$, $k), \widehat{m}_{k}, \beta_{3}\left(\widehat{m}_{k}, k\right), \gamma_{3,1}\left(\widehat{m}_{k}, k\right), \gamma_{3,2}\left(\widehat{m}_{k}, k\right)$, and $\gamma_{3,3}\left(\widehat{m}_{k}, k\right)$, for each of the energy commodity data sets. According to (58), the estimate $\gamma_{j, l}\left(\widehat{m}_{k}, k\right), j \neq l$, is positive if 


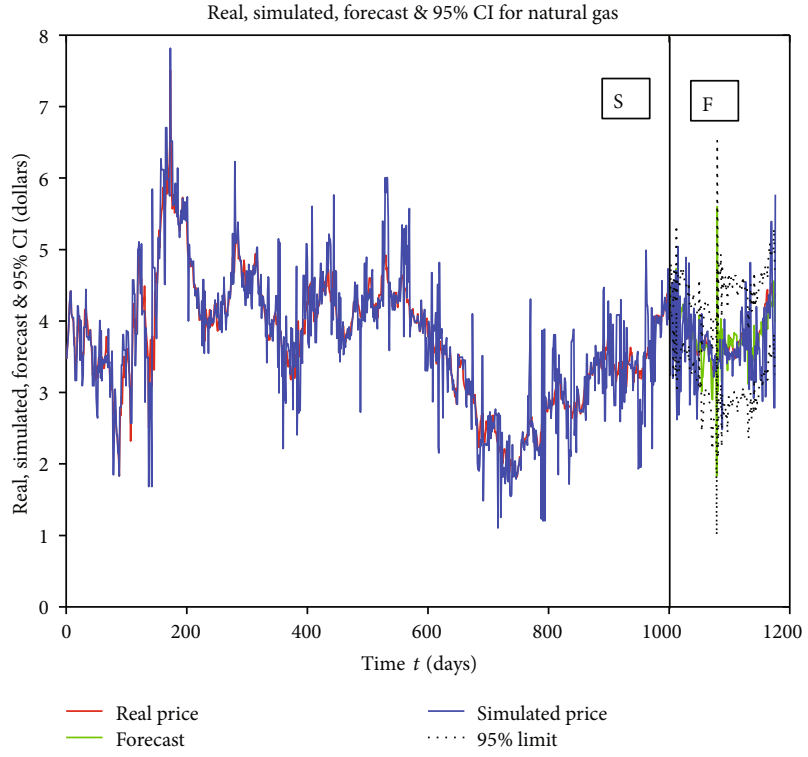

(a)

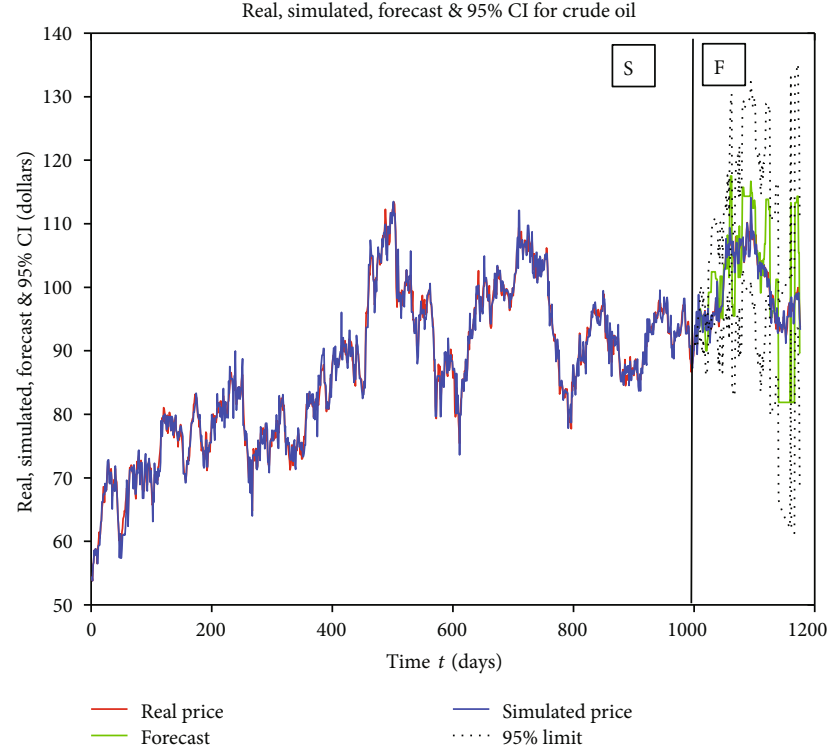

(b)

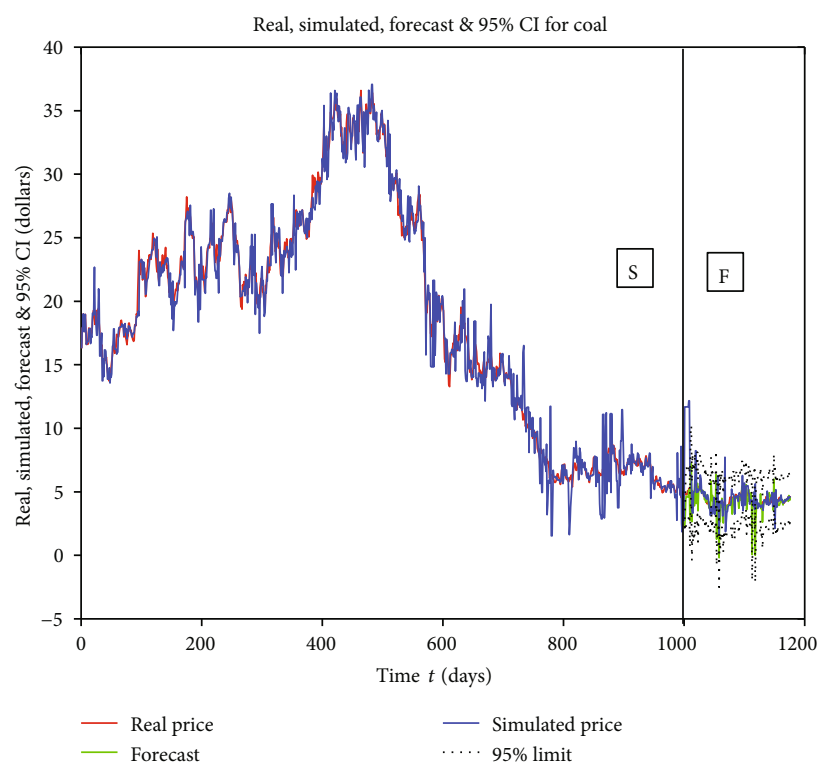

(c)

FIgURE 15: Real, simulated, forecasted prices and 95\% C.I. with no jump.

commodity $p_{l}$ is cooperating with commodity $p_{j}$, and negative if commodity $p_{l}$ is competing with commodity $p_{j}$. There is no interaction between the two commodities if $\gamma_{j, l}$ $\left(\widehat{m}_{k}, k\right)=0$. It is apparent from the graph (from $\gamma_{1,3}\left(\widehat{m}_{k}, k\right)$ in Column 6) that coal is competing with natural gas during this period because the estimates of $\gamma_{1,3}\left(\widehat{m}_{k}, k\right)$ are mostly negative. It is apparent that natural gas and crude oil are either cooperating or competing, depending on the time period.

The graph of the drift coefficient's parameters with estimates in Table 10 are given in Figure 11 below.

Figures 11(a)-11(i) show the graph of the $\varepsilon$ suboptimal interaction coefficient parameters $\gamma_{1,1}\left(\widehat{m}_{k}, k\right), \gamma_{1,2}\left(\widehat{m}_{k}, k\right)$, $\gamma_{1,3}\left(\widehat{m}_{k}, k\right), \gamma_{2,1}\left(\widehat{m}_{k}, k\right), \gamma_{2,2}\left(\widehat{m}_{k}, k\right), \gamma_{2,3}\left(\widehat{m}_{k}, k\right), \gamma_{3,1}\left(\widehat{m}_{k}, k\right)$, $\gamma_{3,2}\left(\hat{m}_{k}, k\right)$, and $\gamma_{3,3}\left(\widehat{m}_{k}, k\right)$. According to (58), the estimate $\gamma_{j, l}\left(\widehat{m}_{k}, k\right), j \neq l$, is positive if commodity $p_{l}$ is cooperating with commodity $p_{j}$, and negative if commodity $p_{l}$ is competing with commodity $p_{j}$. There is no interaction between the two commodities if $\gamma_{j, l}\left(\widehat{m}_{k}, k\right)=0$. It is apparent from the graph of $\gamma_{1,3}\left(\widehat{m}_{k}, k\right)$ that coal is competing with natural gas because the estimates of $\gamma_{1,3}\left(\hat{m}_{k}, k\right)$ are mostly negative. This shows that for fixed price $p_{1}$ of natural gas, for every $\$ 1$ increase in the price of coal, the price of natural gas decreases on the average by 0.0019 . Also, it is apparent that natural gas and crude oil are either cooperating or competing, depending on the time period. 


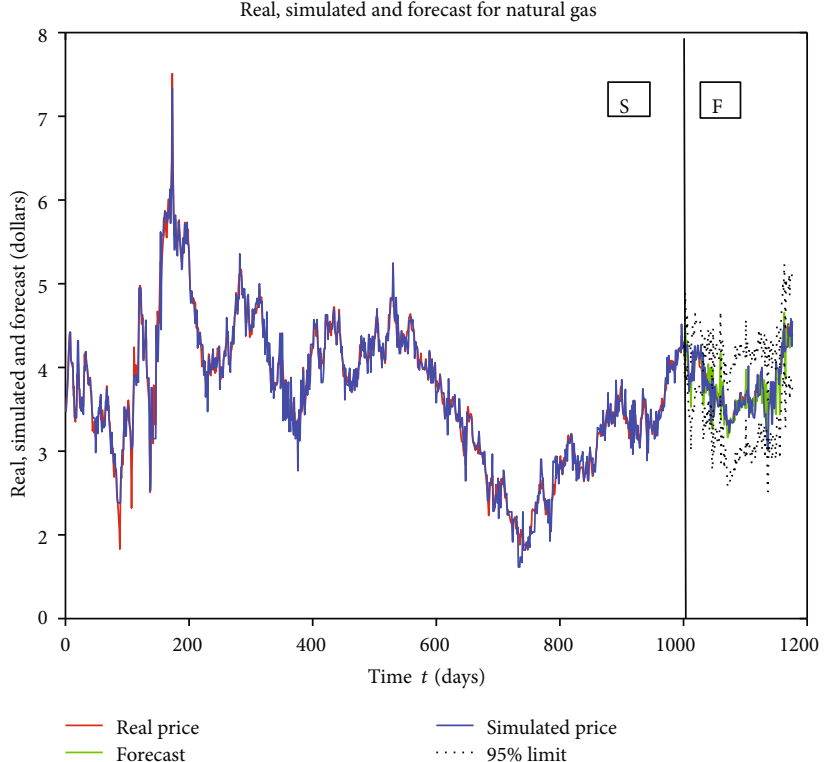

(a)

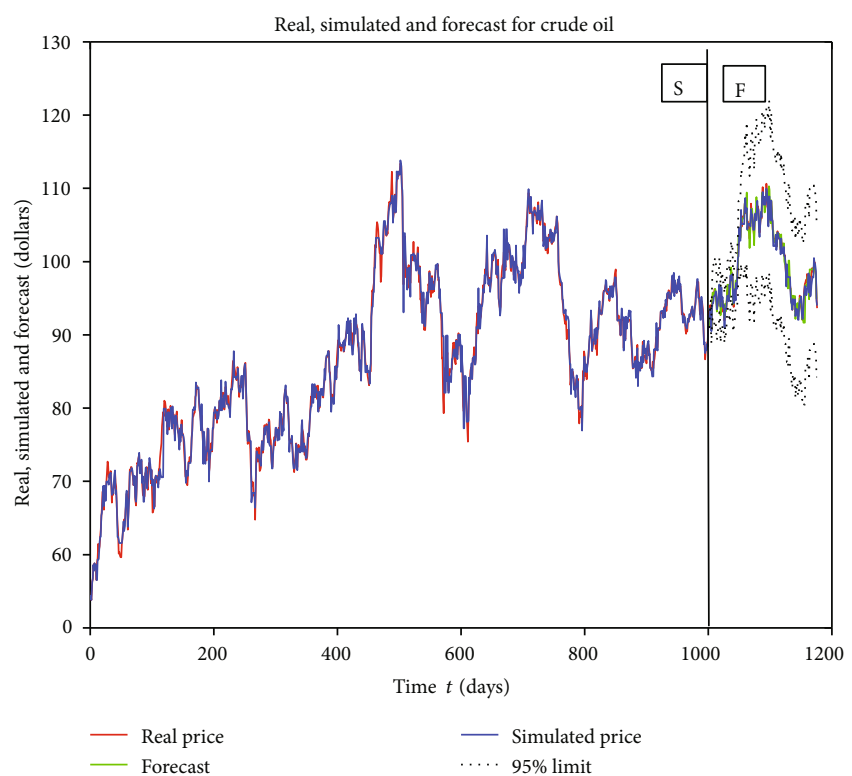

(b)

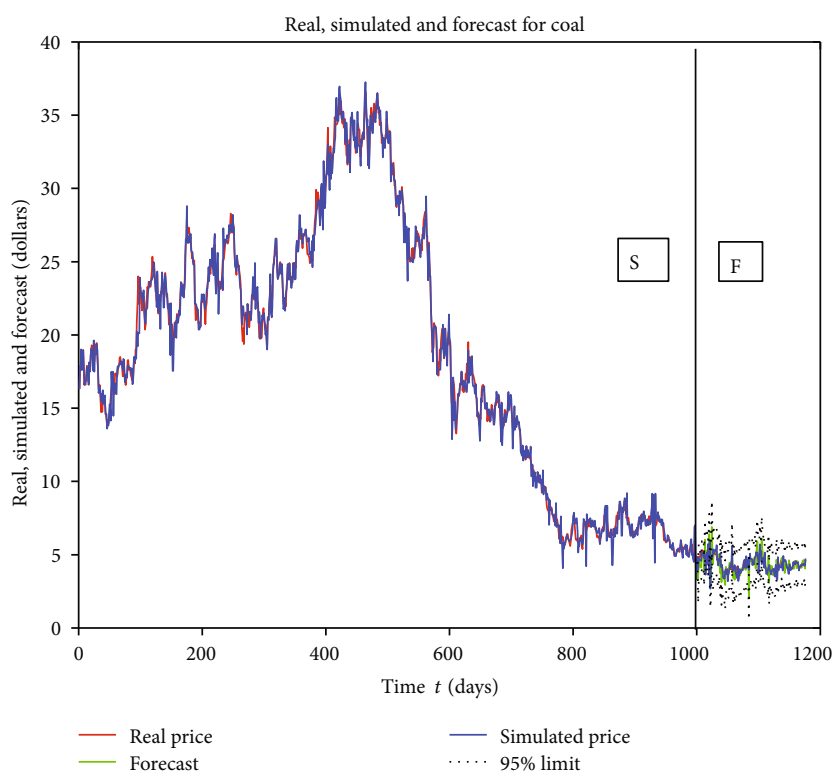

(c)

FIGURE 16: Real, simulated, forecasted prices and 95\% CI with jump.

Figures 12(a)-12(c) are the graphs of $\beta_{1}\left(\widehat{m}_{k}, k\right), \beta_{2}\left(\widehat{m}_{k}\right.$, $k)$, and $\beta_{3}\left(\widehat{m}_{k}, k\right)$ against time $t_{k}$ for the daily Henry Hub natural gas price data set [36], daily crude oil price data set [37], and daily coal price data set, respectively.

Table 11 gives the $\varepsilon$ suboptimal estimates of the parameters $u_{1}\left(\widehat{m}_{k}, k\right), \sigma_{1,1}\left(\widehat{m}_{k}, k\right), \sigma_{1,2}\left(\widehat{m}_{k}, k\right), \sigma_{1,3}\left(\widehat{m}_{k}, k\right), u_{2}\left(\widehat{m}_{k}, k\right)$, $\sigma_{2,1}\left(\widehat{m}_{k}, k\right), \sigma_{2,2}\left(\widehat{m}_{k}, k\right), \sigma_{2,3}\left(\widehat{m}_{k}, k\right), u_{3}\left(\widehat{m}_{k}, k\right), \sigma_{3,1}\left(\widehat{m}_{k}, k\right)$, $\sigma_{3,2}\left(\widehat{m}_{k}, k\right)$, and $\sigma_{3,3}\left(\widehat{\mathrm{m}}_{k}, k\right)$ for each of the energy commodity data sets.

Figures 13(a)-13(c) are the graphs of $\sigma_{1,1}\left(\widehat{m}_{k}, k\right), \sigma_{1,2}$ $\left(\widehat{m}_{k}, k\right), \sigma_{1,3}\left(\widehat{m}_{k}, k\right), \sigma_{2,1}\left(\widehat{m}_{k}, k\right), \sigma_{2,2}\left(\widehat{m}_{k}, k\right), \sigma_{2,3}\left(\widehat{m}_{k}, k\right), \sigma_{3,1}$ $\left(\widehat{m}_{k}, k\right), \sigma_{3,2}\left(\widehat{m}_{k}, k\right)$, and $\sigma_{3,3}\left(\widehat{m}_{k}, k\right)$ against time $t_{k}$ for the daily Henry Hub natural gas price data set [36], daily crude oil price data set [37], and daily coal price data set, respectively.

Table 12 shows the real and simulated estimates for the spot price processes $p_{j}(t), j \in I(1, n)$.

Figure 14 shows the graph of the real and simulated prices (with jump) for natural gas, crude oil, and coal data set.

Figures 14(a)-14(c) show the graph of the real and simulated spot prices for the daily Henry Hub natural gas data set [36], daily crude oil data set [37], and daily coal data set [35], respectively. The red line represents the real data set $\mathbf{p}$, while the blue line represent the 
simulated data set $\mathbf{p}_{\widehat{m}_{k}, k}^{s}$. It is clear that the graph fits well. To reduce magnitude of error, we increase the magnitude of time delay. It is obvious that these curves fit better than the curves in Figure 7. It follows that the interconnected dynamical system with jump process incorporated into it performs better than the one without jump.

\section{Forecasting}

In this section, we shall sketch an outline about forecasting problem for the case where there is no jump. The sketch for the case where jump exist is similar. An $\varepsilon$ suboptimal simulated value $\left(\mathbf{y}^{s}\left(\widehat{m}_{k}^{i-1}, t_{k}^{i-1}\right), \mathbf{p}^{s}\left(\widehat{m}_{k}^{i-1}, t_{k}^{i-1}\right)\right)$ at time $t_{k}^{i-1}, i \in I\left(1, K^{*}\right)$, is used to define a forecast $\left(\mathbf{y}^{f}\left(\widehat{m}_{k}^{i-1}, t_{k}^{i-1}\right), \mathbf{p}^{f}\left(\widehat{m}_{k}^{i-1}, t_{k}^{i-1}\right)\right)$ for $\left(\mathbf{y}\left(t_{k}^{i-1}\right), \mathbf{p}\left(t_{k}^{i-1}\right)\right)$ at the time $t_{k}^{i-1}$ for the system of energy commodity model.

8.1. Forecasting for Energy Commodity Model. In the context of Section 6.1, for $i \in I\left(1, K^{*}\right)$, we begin forecasting from time $t_{k}^{i-1}$. Using the data set up to time $t_{k-1}^{i-1}$, we compute $\widehat{m}_{a}^{i-1}, \widehat{m}_{a}^{i-1}$, $u_{j}\left(\widehat{m}_{a}^{i-1}, t_{a}^{i-1}\right), \quad \beta_{j}\left(\widehat{m}_{a}^{i-1}, t_{a}^{i-1}\right), \quad \kappa_{j, l}\left(\widehat{m}_{a}^{i-1}, t_{a}^{i-1}\right), \quad \gamma_{j, l}\left(\widehat{m}_{a}^{i-1}, t_{a}^{i-1}\right)$, $\delta_{j, l}\left(\widehat{m}_{a}^{i-1}, t_{a}^{i-1}\right), \sigma_{j, l}\left(\widehat{m}_{a}^{i-1}, t_{a}^{i-1}\right)$, and $j, l \in I(1,3)$ for $a \in I(0, k$ $-1)$. We assume that we have no information about the real data set $\left\{y_{j}\left(t_{a}^{i-1}\right)\right\}_{a=k}^{N_{i-1}}$. Under these considerations, imitating the computational procedure outlined in Section 7 and using solutions to (66) and (67), we find the estimate of the forecast $\mathbf{y}^{f}\left(\widehat{m}_{k}^{i-1}, t_{k}^{i-1}\right)$ and $\mathbf{p}^{f}\left(\widehat{m}_{k}^{i-1}, t_{k}^{i-1}\right)$ at time $t_{k}^{i-1}$ as follows:

$$
\left\{\begin{aligned}
y_{j}^{f}\left(\widehat{m}_{k}^{i-1}, t_{k}^{i-1}\right)= & y_{j}^{s}\left(\widehat{m}_{k-1}^{i-1}, t_{k-1}^{i-1}\right)+\left(u_{j}\left(\widehat{m}_{k-1}^{i-1}, t_{k-1}^{i-1}\right)-y_{j}^{s}\left(\widehat{m}_{k-1}^{i-1}, t_{k-1}^{i-1}\right)\right)\left[\kappa_{j, j}\left(\widehat{m}_{k-1}^{i-1}, t_{k-1}^{i-1}\right) y_{j}^{s}\left(\widehat{m}_{k-1}^{i-1}, t_{k-1}^{i-1}\right)+\sum_{l \neq j}^{n} \kappa_{j,} y_{l}^{s}\left(\widehat{m}_{k-1}^{i-1}, t_{k-1}^{i-1}\right)\right] \Delta t \\
& +\delta_{j, j}\left(\widehat{m}_{k-1}^{i-1}, t_{k-1}^{i-1}\right)\left(u_{j}\left(\widehat{m}_{k-1}^{i-1}, t_{k-1}^{i-1}\right)-y_{j}^{s}\left(\widehat{m}_{k-1}^{i-1}, t_{k-1}^{i-1}\right)\right) W_{j, j}(k)+\left(u_{j}\left(\widehat{m}_{k-1}^{i-1}, t_{k-1}^{i-1}\right)-y_{j}^{s}\left(\widehat{m}_{k-1}^{i-1}, t_{k-1}^{i-1}\right)\right) \sum_{l \neq j}^{n} \delta_{j, l} y_{l}^{s}\left(\widehat{m}_{k-1}^{i-1}, t_{k-1}^{i-1}\right) W_{j, l}(k), \\
p_{j}^{f}\left(\widehat{m}_{k}^{i-1}, t_{k}^{i-1}\right)= & p_{j}^{s}\left(\widehat{m}_{k-1}^{i-1}, t_{k-1}^{i-1}\right)+p_{j}^{s}\left(\widehat{m}_{k-1}^{i-1}, t_{k-1}^{i-1}\right)\left[\gamma_{j, j}\left(\widehat{m}_{k-1}^{i-1}, t_{k-1}^{i-1}\right)\left(y_{j}^{s}\left(\widehat{m}_{k-1}^{i-1}, t_{k-1}^{i-1}\right)-p_{j}^{s}\left(\widehat{m}_{k-1}^{i-1}, t_{k-1}^{i-1}\right)\right)+\beta_{j}\left(\widehat{m}_{k-1}^{i-1}, t_{k-1}^{i-1}\right)+\sum_{l \neq j}^{n} \gamma_{j, l}\left(\widehat{m}_{k-1}^{i-1}, t_{k-1}^{i-1}\right) p_{l}^{s}\left(\widehat{m}_{k-1}^{i-1}, t_{k-1}^{i-1}\right)\right] \Delta t \\
& +\sigma_{j, j}\left(\widehat{m}_{k-1}^{i-1}, t_{k-1}^{i-1}\right) p_{j}^{s}\left(\widehat{m}_{k-1}^{i-1}, t_{k-1}^{i-1}\right) Z_{j, j}(k)+p_{j}^{s}\left(\widehat{m}_{k-1}^{i-1}, t_{k-1}^{i-1}\right) \sum_{l \neq j}^{n} \sigma_{j, l}\left(\widehat{m}_{k-1}^{i-1}, t_{k-1}^{i-1}\right) p_{l}^{s}\left(\widehat{m}_{k-1}^{i-1}, t_{k-1}^{i-1}\right) Z_{j, l}(k), t_{k}^{i-1} \in\left[T_{i-1}, T_{i}\right) .
\end{aligned}\right.
$$

To find the forecasted value $y_{j}^{f}\left(T_{i}^{-}\right)$and $p_{j}^{f}\left(T_{i}^{-}\right)$, we need to re-estimate $\widehat{\pi}_{j}^{i}$ and $\widehat{\theta}_{j}^{i}$ by first simulating

$$
\begin{gathered}
\lim _{t \rightarrow T_{i}^{-}} y_{j}^{f}\left(t, T_{i-1}, \mathbf{y}^{i-1, s}\right) \equiv y_{j}^{f}\left(m_{N_{i-1}}^{i-1}, t_{N_{i-1}}^{i-1}\right), \\
\lim _{t \rightarrow T_{i}^{-}} p_{j}^{f}\left(t, T_{i-1}, \mathbf{y}^{i-1, s}, \mathbf{p}^{i-1, s}\right) \equiv p_{j}^{f}\left(m_{N_{i-1}}^{i-1}, t_{N_{i-1}}^{i-1}\right),
\end{gathered}
$$

as follows:

$$
\begin{aligned}
y_{j}^{f}\left(m_{N_{i-1}}^{i-1}, t_{N_{i-1}}^{i-1}\right)= & y_{j}^{s}\left(m_{N_{i-1}-1}^{i-1}, t_{N_{i-1}-1}^{i-1}\right)+\left(u_{j}^{i-1}\left(m_{N_{i-1}-1}^{i-1}, t_{N_{i-1}-1}^{i-1}\right)\right. \\
& \left.-y_{j}^{s}\left(m_{N_{i-1}-1}^{i-1}, t_{N_{i-1}-1}^{i-1}\right)\right)\left[\sum_{l=1}^{n} \kappa_{j, l}^{i-1}\left(m_{N_{i-1}-1}^{i-1}, t_{N_{i-1}-1}^{i-1}\right)\right. \\
& \left.\cdot y_{l}^{s}\left(m_{N_{i-1}-1}^{i-1}, t_{N_{i-1}-1}^{i-1}\right)\right] \Delta t+\left(u_{j}^{i-1}\left(m_{N_{i-1}-1}^{i-1}, t_{N_{i-1}-1}^{i-1}\right)\right. \\
& \left.-y_{j}^{s}\left(m_{N_{i-1}-1}^{i-1}, t_{N_{i-1}-1}^{i-1}\right)\right)\left[\delta_{j, j}^{i-1}\left(m_{N_{i-1}-1}^{i-1}, t_{N_{i-1}-1}^{i-1}\right)\right. \\
& \cdot \Delta W_{j, j}\left(m_{N_{i-1}}^{i-1}, t_{N_{i-1}}^{i-1}\right)+\sum_{l \neq j}^{n} \delta_{j, l}^{i-1}\left(m_{N_{i-1}-1}^{i-1}, t_{N_{i-1}-1}^{i-1}\right) \\
& \left.\cdot y_{l}^{s}\left(m_{N_{i-1}-1}^{i-1}, t_{N_{i-1}-1}^{i-1}\right) \Delta W_{j, l}\left(m_{N_{i-1}}^{i-1}, t_{N_{i-1}}^{i-1}\right)\right],
\end{aligned}
$$

$$
\begin{aligned}
p_{j}^{f}\left(m_{N_{i-1}}^{i-1}, t_{N_{i-1}}^{i-1}\right)= & p_{j}^{s}\left[\gamma _ { j , j } ^ { i - 1 } ( m _ { N _ { i - 1 } - 1 } ^ { i - 1 } , t _ { N _ { i - 1 } - 1 } ^ { i - 1 } ) \left(y_{j}^{s}\left(m_{N_{i-1}-1}^{i-1}, t_{N_{i-1}-1}^{i-1}\right)\right.\right. \\
& \left.-p_{j}^{s}\left(m_{N_{i-1}-1}^{\mathrm{i}-1}, t_{N_{i-1}-1}^{i-1}\right)\right)+\beta_{j}^{i-1}\left(m_{N_{i-1}-1}^{i-1}, t_{N_{i-1}-1}^{i-1}\right) \\
& \left.+\sum_{l \neq j}^{n} \gamma_{j, l}^{i-1}\left(m_{N_{i-1}-1}^{i-1}, t_{N_{i-1}-1}^{i-1}\right) p_{l}^{s}\left(m_{N_{i-1}-1}^{i-1}, t_{N_{i-1}-1}^{i-1}\right)\right] \Delta t \\
& +\sigma_{j, j}^{i-1}\left(m_{N_{i-1}-1}^{i-1}, t_{N_{i-1}-1}^{i-1}\right) p_{j}^{s}\left(m_{N_{i-1}-1}^{i-1}, t_{N_{i-1}-1}^{i-1}\right) \\
& \cdot \Delta Z_{j, j}\left(m_{N_{i-1}}^{i-1}, t_{N_{i-1}}^{i-1}\right)+p_{j}^{s}\left(m_{N_{i-1}-1}^{i-1}, t_{N_{i-1}-1}^{i-1}\right) \\
& \cdot \sum_{l \neq j}^{n} \sigma_{j, l}^{i-1}\left(m_{N_{i-1}-1}^{i-1}, t_{N_{i-1}-1}^{i-1}\right) p_{l}^{s}\left(m_{N_{i-1}-1}^{i-1}, t_{N_{i-1}-1}^{i-1}\right) \\
& \cdot \Delta Z_{j, l}\left(m_{N_{i-1}}^{i-1}, t_{N_{i-1}}^{i-1}\right), t_{k}^{i-1}=t_{N_{i-1}}^{i-1} .
\end{aligned}
$$

Imitating argument in (84), we define

$$
\begin{gathered}
\widehat{\pi}_{j}^{i}=\frac{y_{j}^{s}\left(m_{N_{i-1}}^{i-1}, t_{N_{i-1}}^{i-1}\right)}{y_{j}^{f}\left(m_{N_{i-1}}^{i-1}, t_{N_{i-1}}^{i-1}\right)}, \\
\widehat{\theta}_{j}^{i}=\frac{p_{j}^{s}\left(m_{N_{i-1}}^{i-1}, t_{N_{i-1}}^{i-1}\right)}{p_{j}^{f}\left(m_{N_{i-1}}^{i-1}, t_{N_{i-1}}^{i-1}\right)} .
\end{gathered}
$$


Thus, $y_{j}^{f}\left(\boldsymbol{T}_{i}^{-}\right)=\widehat{\pi}_{j}^{i} y_{j}^{f}\left(\boldsymbol{T}_{i}^{-}, \boldsymbol{T}_{i-1}, \mathbf{y}^{i-1, s}\right)$ and $p_{j}^{f}\left(T_{i}^{-}\right)=\widehat{\theta}_{j}^{i} p_{j}^{f}$ $\left(T_{i}^{-}, T_{i-1}, \mathbf{y}^{i-1, f}, \mathbf{p}^{i-1, f}\right)$. where the estimates $u_{j}\left(\widehat{m}_{k-1}^{i-1}, t_{k-1}^{i-1}\right), \beta_{j}$ $\left(\widehat{m}_{k-1}^{i-1}, t_{k-1}^{i-1}\right), \kappa_{j, l}\left(\widehat{m}_{k-1}^{i-1}, t_{k-1}^{i-1}\right), \gamma_{j, l}\left(\widehat{m}_{k-1}^{i-1}, t_{k-1}^{i-1}\right), \quad \delta_{j, l}\left(\widehat{m}_{k-1}^{i-1}, t_{k-1}^{i-1}\right)$, and $\sigma_{j, l}\left(\widehat{m}_{k-1}^{i-1}, t_{k-1}^{i-1}\right), j, l \in I(1,3)$ are estimated with respect to the known past data set up to the time $t_{k-1}^{i-1}$. We note that $\mathbf{y}_{\hat{m}_{k}^{i-1}, t_{k}^{i-1}}^{f}$ is the $\varepsilon$ suboptimal estimate for $\mathbf{y}_{j}\left(t_{k}^{i-1}\right)$ at time $t_{k}^{i-1}$.
To determine $\left(\mathbf{y}^{f}\left(\widehat{m}_{k+1}^{i-1}, t_{k+1}^{i-1}\right), \mathbf{p}^{f}\left(\widehat{m}_{k+1}^{i-1}, t_{\mathrm{k}+1}^{i-1}\right)\right)$, we need $u_{j}\left(\widehat{m}_{k}^{i-1}, t_{k}^{i-1}\right), \quad \beta_{j}\left(\widehat{m}_{k}^{i-1}, t_{k}^{i-1}\right), \quad \kappa_{j, l}\left(\widehat{m}_{k}^{i-1}, t_{k}^{i-1}\right), \quad \gamma_{j, l}\left(\widehat{m}_{k}^{i-1}, t_{k}^{i-1}\right)$, $\delta_{j, l}\left(\widehat{m}_{k}^{i-1}, t_{k}^{i-1}\right)$, and $\sigma_{j, l}\left(\widehat{m}_{k}^{i-1}, t_{k}^{i-1}\right), j, l \in I(1,3)$. Since we only have information of real data up to time $t_{k-1}$, we use the forecasted estimate $y_{j}^{f}\left(\widehat{m}_{k}^{i-1}, t_{k}^{i-1}\right)$ as the estimate of $y_{j}\left(t_{k}^{i-1}\right)$ at time $t_{k}^{i-1}$ and to estimate $u_{j}\left(\widehat{m}_{k}^{i-1}, t_{k}^{i-1}\right), \beta_{j}\left(\widehat{m}_{k}^{i-1}, t_{k}^{i-1}\right), \kappa_{j, l}$ $\left(\widehat{m}_{k}^{i-1}, t_{k}^{i-1}\right), \gamma_{j, l}\left(\widehat{m}_{k}^{i-1}, t_{k}^{i-1}\right), \delta_{j, l}\left(\widehat{m}_{k}^{i-1}, t_{k}^{i-1}\right)$, and $\sigma_{j, l}\left(\widehat{m}_{k}^{i-1}, t_{k}^{i-1}\right)$, $j, l \in I(1,3)$. Hence, we can write $u_{j}\left(\widehat{m}_{k}^{i-1}, t_{k}^{i-1}\right)$ as

$$
\left\{\begin{array}{l}
u_{j}\left(\widehat{m}_{k}^{i-1}, t_{k}^{i-1}\right) \equiv u_{j}\left(\widehat{m}_{k}^{i-1}, y_{j}\left(t_{k-\widehat{m}_{k}^{i-1}+1}^{i-1}\right), y_{j}\left(t_{k-\widehat{m}_{k}^{i-1}+2}^{i-1}\right), \cdots, y_{j}\left(t_{k-1}^{i-1}\right), y_{j}^{f}\left(\widehat{m}_{k}^{i-1}, t_{k}^{i-1}\right)\right) \\
\kappa_{j, l}\left(\widehat{m}_{k}^{i-1}, t_{k}^{i-1}\right) \equiv \kappa_{j, l}\left(\widehat{m}_{k}^{i-1}, y_{j}\left(t_{k-\widehat{m}_{k}^{i-1}+1}^{i-1}\right), y_{j}\left(t_{k-\widehat{m}_{k}^{i-1}+2}^{i-1}\right), \cdots, y_{j}\left(t_{k-1}^{i-1}\right), y_{j}^{f}\left(\widehat{m}_{k}^{i-1}, t_{k}^{i-1}\right)\right) \\
\delta_{j, l}\left(\widehat{m}_{k}^{i-1}, t_{k}^{i-1}\right) \equiv \delta_{j, l}\left(\widehat{m}_{k}^{i-1}, y_{j}\left(t_{k-\widehat{m}_{k}^{i-1}+1}^{i-1}\right), y_{j}\left(t_{k-\widehat{m}_{k}^{i-1}+2}^{i-1}\right), \cdots, y_{j}\left(t_{k-1}^{i-1}\right), y_{j}^{f}\left(\widehat{m}_{k}^{i-1}, t_{k}^{i-1}\right)\right), \\
\beta_{j, l}\left(\widehat{m}_{k}^{i-1}, t_{k}^{i-1}\right) \equiv \beta_{j, l}\left(\widehat{m}_{k}^{i-1}, p_{j}\left(t_{k-\widehat{m}_{k}^{i-1}+1}^{i-1}\right), p_{j}\left(t_{k-\widehat{m}_{k}^{i-1}+2}^{i-1}\right), \cdots, p_{j}\left(t_{k-1}^{i-1}\right), p_{j}^{f}\left(\widehat{m}_{k}^{i-1}, t_{k}^{i-1}\right)\right) \\
\gamma_{j, l}\left(\widehat{m}_{k}^{i-1}, t_{k}^{i-1}\right) \equiv \gamma_{j, l}\left(\widehat{m}_{k}^{i-1}, p_{j}\left(t_{k-\widehat{m}_{k}^{i-1}+1}^{i-1}\right), p_{j}\left(t_{k-\widehat{m}_{k}^{i-1}+2}^{i-1}\right), \cdots, p_{j}\left(t_{k-1}^{i-1}\right), p_{j}^{f}\left(\widehat{m}_{k}^{i-1}, t_{k}^{i-1}\right)\right), \\
\sigma_{j, l}\left(\widehat{m}_{k}^{i-1}, t_{k}^{i-1}\right) \equiv \sigma_{j, l}\left(\widehat{m}_{k}^{i-1}, p_{j}\left(t_{k-\hat{m}_{k}^{i-1}+1}^{i-1}\right), p_{j}\left(t_{k-\widehat{m}_{k}^{i-1}+2}^{i-1}\right), \cdots, p_{j}\left(t_{k-1}^{i-1}\right), p_{j}^{f}\left(\widehat{m}_{k}^{i-1}, t_{k}^{i-1}\right)\right), j, l \in I(1, n) .
\end{array}\right.
$$

For $j, l \in I(1, n)$, to find $\left(y_{j}^{f}\left(\widehat{m}_{k+2}^{i-1}, t_{k+2}^{i-1}\right), p_{j}^{f}\left(\widehat{m}_{k+2}^{i-1}, t_{k+2}^{i-1}\right)\right)$, we use the estimates

$$
\left\{\begin{array}{l}
u_{j}\left(\widehat{m}_{k+1}^{i-1}, t_{k+1}^{i-1}\right) \equiv u_{j}\left(\widehat{m}_{k+1}^{i-1}, y_{j}\left(t_{k-\widehat{m}_{k}^{i-1}+2}^{i-1}\right), y_{j}\left(t_{k-\hat{m}_{k}^{i-1}+3}^{i-1}\right), \cdots, y_{j}\left(t_{k-1}^{i-1}\right), y_{j}^{f}\left(\widehat{m}_{k}^{i-1}, t_{k}^{i-1}\right), y_{j}^{f}\left(\widehat{m}_{k+1}^{i-1}, t_{k+1}^{i-1}\right)\right), \\
\kappa_{j, l}\left(\widehat{m}_{k+1}^{i-1}, t_{k+1}^{i-1}\right) \equiv \kappa_{j, l}\left(\widehat{m}_{k+1}^{i-1}, y_{j}\left(t_{k-\widehat{m}_{k}^{i-1}+2}^{i-1}\right), y_{j}\left(t_{k-\widehat{m}_{k}^{i-1}+3}^{i-1}\right), \cdots, y_{j}\left(t_{k-1}^{i-1}\right), y_{j}^{f}\left(\widehat{m}_{k}^{i-1}, t_{k}^{i-1}\right), y_{j}^{f}\left(\widehat{m}_{k+1}^{i-1}, t_{k+1}^{i-1}\right)\right), \\
\delta_{j, l}\left(\widehat{m}_{k+1}^{i-1}, t_{k+1}^{i-1}\right) \equiv \delta_{j, l}\left(\widehat{m}_{k+1}^{i-1}, y_{j}\left(t_{k-\widehat{m}_{k}^{i-1}+2}^{i-1}\right), y_{j}\left(t_{k-\widehat{m}_{k}^{i-1}+3}^{i-1}\right), \cdots, y_{j}\left(t_{k-1}^{i-1}\right), y_{j}^{f}\left(\widehat{m}_{k}^{i-1}, t_{k}^{i-1}\right), y_{j}^{f}\left(\widehat{m}_{k+1}^{i-1}, t_{k+1}^{i-1}\right)\right), \\
\beta_{j, l}\left(\widehat{m}_{k+1}^{i-1}, t_{k+1}^{i-1}\right) \equiv \beta_{j, l}\left(\widehat{m}_{k+1}^{i-1}, p_{j}\left(t_{k-\widehat{m}_{k}^{i-1}+2}^{i-1}\right), p_{j}\left(t_{k-\widehat{m}_{k}^{i-1}+3}^{i-1}\right), \cdots, p_{j}\left(t_{k-1}^{i-1}\right), p_{j}^{f}\left(\widehat{m}_{k}^{i-1}, t_{k}^{i-1}\right), p_{j}^{f}\left(\widehat{m}_{k+1}^{i-1}, t_{k+1}^{i-1}\right)\right), \\
\gamma_{j, l}\left(\widehat{m}_{k+1}^{i-1}, t_{k+1}^{i-1}\right) \equiv \gamma_{j, l}\left(\widehat{m}_{k+1}^{i-1}, p_{j}\left(t_{k-\widehat{m}_{k}^{i-1}+2}^{i-1}\right), p_{j}\left(t_{k-\widehat{m}_{k}^{i-1}+3}^{i-1}\right), \cdots, p_{j}\left(t_{k-1}^{i-1}\right), p_{j}^{f}\left(\widehat{m}_{k}^{i-1}, t_{k}^{i-1}\right), p_{j}^{f}\left(\widehat{m}_{k+1}^{i-1}, t_{k+1}^{i-1}\right)\right), \\
\sigma_{j, l}\left(\widehat{m}_{k+1}^{i-1}, t_{k+1}^{i-1}\right) \equiv \sigma_{j, l}\left(\widehat{m}_{k+1}^{i-1}, p_{j}\left(t_{k-\widehat{m}_{k}^{i-1}+2}^{i-1}\right), p_{j}\left(t_{k-\widehat{m}_{k}^{i-1}+3}^{i-1}\right), \cdots, p_{j}\left(t_{k-1}^{i-1}\right), p_{j}^{f}\left(\widehat{m}_{k}^{i-1}, t_{k}^{i-1}\right), p_{j}^{f}\left(\widehat{m}_{k+1}^{i-1}, t_{k+1}^{i-1}\right)\right) .
\end{array}\right.
$$


Continuing this process in this manner, to find $\left(y_{j}^{f}\left(\widehat{m}_{k+l}^{i-1}\right.\right.$ ,$\left.\left.t_{k+l}^{i-1}\right), p_{j}^{f}\left(\widehat{m}_{k+l}^{i-1}, t_{k+l}^{i-1}\right)\right)$, we use the estimates

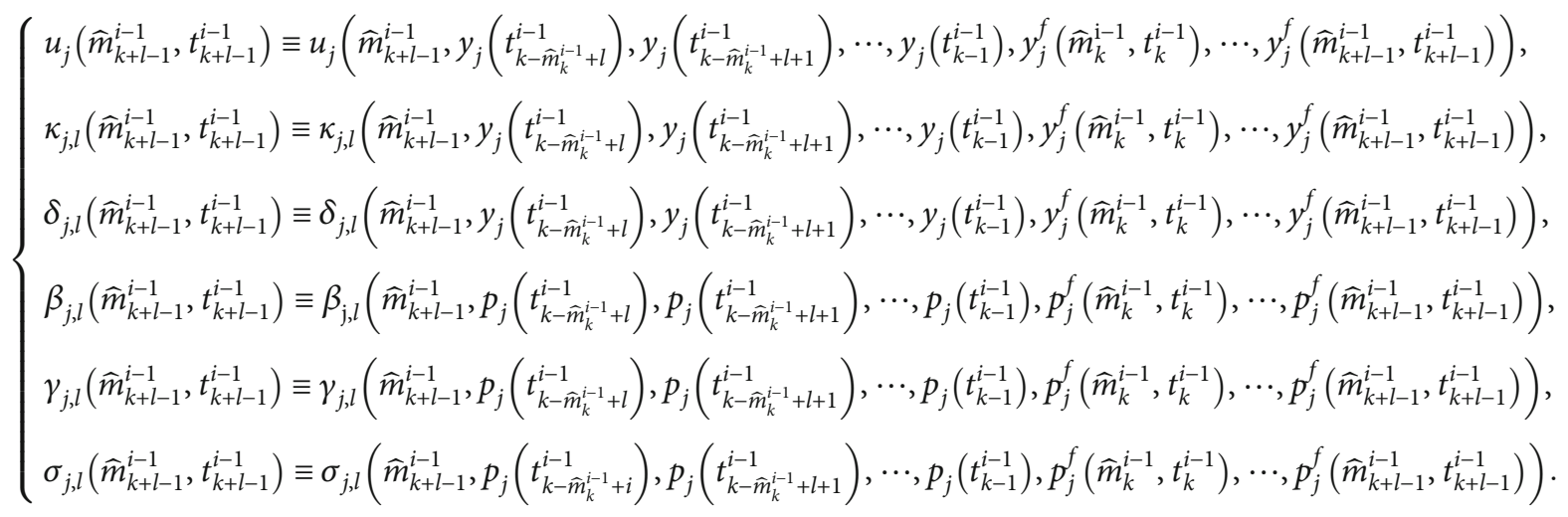

8.1.1. Prediction/Confidence Interval for Energy Commodities. In order to be able to assess the future certainty, we also discuss about the prediction/confidence interval. We define the $100(1-\alpha) \%$ confidence interval for the forecast of the state $\left(\mathbf{y}_{\hat{m}_{l}^{i-1}, t_{l}^{i-1}}^{f}, \mathbf{p}_{\hat{m}_{l}^{i-1}, t_{l}^{i-1}}^{f}\right)$ at time $t_{l}^{i-1}, l \geq k, j \in I(1, n)$ as

$$
\left\{\begin{array}{l}
y_{j}^{f}\left(\widehat{m}_{l}^{i-1}, t_{l}^{i-1}\right) \pm z_{1-\alpha / 2}\left(s_{m \wedge_{l-1}^{j, j}, t_{l-1}^{i-1}}^{i-1}\left(y_{j}^{f}\right)\right)^{1 / 2} \\
p_{j}^{f}\left(\widehat{m}_{l}^{i-1}, t_{l}^{i-1}\right) \pm z_{1-\alpha / 2}\left(s_{m \wedge_{l-1}^{j, j}}^{i_{l-1}, t_{l-1}^{i-1}}\left(p_{j}^{f}\right)\right)^{1 / 2}
\end{array}\right.
$$

where $\left(s^{j, j}\left(m \wedge_{l-1}^{i-1}, t_{l-1}^{i-1}\right)\right)^{1 / 2}$ is the estimate for the sample standard deviation for the forecasted state $y_{j}$, and $\left(s^{j, j}\left(m \wedge_{l-1}^{i-1}, t_{l-1}^{i-1}\right)\right)^{1 / 2}$ is the estimate for the sample standard deviation for the forecasted state $p_{j}$ derived from the following iterative process

$$
\begin{aligned}
& \int y_{j}^{f}\left(\widehat{m}_{l}^{i-1}, t_{l}^{i-1}\right)=y_{j}^{f}\left(\hat{m}_{l-1}^{i-1}, t_{l-1}^{i-1}\right)+\left(u_{j}\left(\widehat{m}_{l-1}^{i-1}, t_{l-1}^{i-1}\right)-y_{j}^{f}\left(\widehat{m}_{l-1}^{i-1}, t_{l-1}^{i-1}\right)\right)\left[\kappa_{j, j}\left(\hat{m}_{l-1}^{i-1}, t_{l-1}^{i-1}\right) y_{j}^{f}\left(\hat{m}_{l-1}^{i-1}, t_{l-1}^{i-1}\right)+\sum_{l \neq j}^{n} \kappa_{j, l} y_{l}^{f}\left(\widehat{m}_{l-1}^{i-1}, t_{l-1}^{i-1}\right)\right] \Delta t \\
& +\delta_{j, j}\left(\widehat{m}_{l-1}^{i-1}, t_{l-1}^{i-1}\right)\left(u_{j}\left(\hat{m}_{l-1}^{i-1}, t_{l-1}^{i-1}\right)-y_{j}^{f}\left(\widehat{m}_{l-1}^{i-1}, t_{l-1}^{i-1}\right)\right) W_{j, j}(l)+\left(u_{j}\left(\widehat{m}_{l-1}^{i-1}, t_{l-1}^{i-1}\right)-y_{j}^{f}\left(\widehat{m}_{l-1}^{i-1}, t_{l-1}^{i-1}\right)\right) \sum_{l \neq j}^{n} \delta_{j, l} y_{l}^{f}\left(\widehat{m}_{l-1}^{i-1}, t_{l-1}^{i-1}\right) W_{j, l}(l), \\
& p_{j}^{f}\left(\widehat{m}_{l}^{i-1}, t_{l}^{i-1}\right)=p_{j}^{f}\left(\hat{m}_{l-1}^{i-1}, t_{l-1}^{i-1}\right)+p_{j}^{f}\left(\widehat{m}_{l-1}^{i-1}, t_{l-1}^{i-1}\right)\left[\gamma_{j, j}\left(\hat{m}_{l-1}^{i-1}, t_{l-1}^{i-1}\right)\left(y_{j}^{f}\left(\widehat{m}_{l-1}^{i-1}, t_{l-1}^{i-1}\right)-p_{j}^{f}\left(\widehat{m}_{l-1}^{i-1}, t_{l-1}^{i-1}\right)\right)+\beta_{j}\left(\widehat{m}_{l-1}^{i-1}, t_{l-1}^{i-1}\right)+\sum_{l \neq j}^{n} \gamma_{j, l}\left(\widehat{m}_{l-1}^{i-1}, t_{l-1}^{i-1}\right) p_{l}^{f}\left(\hat{m}_{l-1}^{i-1}, t_{l-1}^{i-1}\right)\right] \Delta t \\
& +\sigma_{j, j}\left(\widehat{m}_{l-1}^{i-1}, t_{l-1}^{i-1}\right) p_{j}^{f}\left(\hat{m}_{l-1}^{i-1}, t_{l-1}^{i-1}\right) Z_{j, j}(l)+p_{j}^{f}\left(\widehat{m}_{l-1}^{i-1}, t_{l-1}^{i-1}\right) \sum_{l \neq j}^{n} \sigma_{j, l}\left(\widehat{m}_{l-1}^{i-1}, t_{l-1}^{i-1}\right) p_{l}^{f}\left(\widehat{m}_{l-1}^{i-1}, t_{l-1}^{i-1}\right) Z_{j, l}(l) .
\end{aligned}
$$

It is clear that the $95 \%$ confidence interval for the forecast at time $t_{k}$ is

$$
\left\{\begin{array}{l}
\left(y_{j}^{f}\left(\widehat{m}_{k}^{i-1}, t_{k}^{i-1}\right)-1.96\left(s_{m \wedge_{k-1}^{i-1}, k-1}^{j, j}\left(y_{j}^{f}\right)\right)^{1 / 2}, y_{j}^{f}\left(\widehat{m}_{k}^{i-1}, t_{k}^{i-1}\right)+1.96\left(s_{m \wedge_{k-1}^{i-1}, k-1}^{j, j}\left(y_{j}^{f}\right)\right)^{1 / 2}\right) \\
\left(p_{j}^{f}\left(\widehat{m}_{k}^{i-1}, t_{k}^{i-1}\right)-1.96\left(s_{m \wedge_{k-1}^{j, j}, k-1}^{j,-1}\left(p_{j}^{f}\right)\right)^{1 / 2}, p_{j}^{f}\left(\widehat{m}_{k}^{i-1}, t_{k}^{i-1}\right)+1.96\left(s_{m \wedge_{k-1}^{j, j}, k-1}^{i-1}\left(p_{j}^{f}\right)\right)^{1 / 2}\right)
\end{array}\right.
$$


where the lower ends denote the lower bounds of the state estimate and the upper ends denote the upper bounds of the state estimate.

\subsubsection{Illustration: Prediction/Confidence Interval for Energy} Commodities with No Jump. For the case of no jump, the following graphs show the simulated, forecasted, and 95 percent confidence limit for the daily Henry Hub natural gas data set [36], daily crude oil data set [37], and daily coal data set [35], respectively.

Figures 15(a)-15(c) show the graph of the forecast and 95 percent confidence limit for the case where there is no jump for the daily Henry Hub natural gas data set [36], daily crude oil data set [37], and daily coal data set [35], respectively. The figure shows two regions: the simulation region $S$ and the forecast region $F$. For the simulation region $S$, we plot the real data set together with the simulated data set as described in Figure 14. For forecast region $F$, we plot the estimate of the forecast as explained in Section 8.1.1.

\subsubsection{Illustration: Prediction/Confidence Interval for Energy} Commodities with Jump. For the jump process, the following graphs show the forecast and 95 percent confidence limit for the daily Henry Hub natural gas data set [36], daily crude oil data set [37], and daily coal data set [35], respectively.

Figures 16(a)-16(c) show the graphs of the forecast and 95 percent confidence limit for the case where there is jump for the daily Henry Hub Natural gas data set [36], daily Crude Oil data set [37], and daily Coal data set [35], respectively. Figures 16(a)-16(c) show two regions: the simulation region $S$ and the forecast region $F$. For the simulation region $S$, we plot the real data set together with the simulated data set as described in Figure 14. For the forecast region $F$, we plot the estimate of the forecast as explained in Section 8.1.1.

\section{Conclusion and Future Work}

We examine the relationship between the prices of the energy commodities: natural gas, crude oil, and coal. To do this, multivariate stochastic models with and without external random interventions are developed to describe the relationship between the price of energy commodities. The timevarying parameters in the stochastic model are estimated using the LLGMM method. Figures 7 and 14 suggest that the model with jump fits better than the model without jump. For $j, l \in I(1,3)$, the estimates for the drift interaction parameters $\gamma_{j, l}\left(\widehat{m}_{k}, t_{k}^{i-1}\right)$ for the case where jump is incorporated suggest that there are definitely interactions between the spot price of these three commodities. As discussed in (57) and (58), the sign of these parameters suggest that there is competition or cooperation between commodities $l$ and $j$. The estimate of the parameter $\gamma_{j, l}\left(\widehat{m}_{k}, t_{k}^{i-1}\right)$ in Tables 3 and 10 and Figures 4 and 11 suggests that these commodities either compete or cooperate with each other depending on the time period. We can also describe the relationship between any two commodity $j$ and $l, j \neq l \in I(1,3)$, based on the overall average $\widehat{\gamma}_{j, l}=1 / N \sum_{k=1}^{N} \gamma_{j, l}\left(\widehat{m}_{k}, t_{k}^{i-1}\right)$. For example, for the case where jump is incorporated, $\widehat{\gamma}_{1,3}=-0.0017$ and $\widehat{\gamma}_{3,1}=-0.0095$. This suggests that on the average, there is competition between these two commodities. Also, $\widehat{\gamma}_{1,2}=0.0018$ and $\bar{\gamma}_{2,1}=0.0083$. This indicates that on the average, there is cooperation between natural gas and crude oil. Finally, $\widehat{\gamma}_{2,3}=-0.0146$ and $\widehat{\gamma}_{3,2}=-0.0013$. Therefore, on the average, there is competition between crude oil and coal. In the future, we plan to apply the local lagged adapted generalized method of moments to interconnected nonlinear stochastic dynamic model for log-spot price, expected log-spot price, and volatility process. Also, we plan to incorporate delay in the multivariate interconnected nonlinear stochastic model. We plan to be able to apply the extended local lagged adapted generalized method of moments to other multivariate interconnected nonlinear dynamic model different from energy commodity model.

\section{Data Availability}

We will provide the data upon request.

\section{Disclosure}

We would also like to mention that this research paper was presented in the Ph.D. Dissertation [15] and at the American Mathematical Society (AMS) conference at San Antonio, Texas.

\section{Conflicts of Interest}

The authors declare that they have no conflicts of interest.

\section{Acknowledgments}

This research is supported by the Mathematical Sciences Division, The U.S. Army Research Office Grant Number W911NF-12-1-0090.

\section{References}

[1] U S Energy Information Administration Website, Annual Energy Outlook, AEO2014 Early Release Overview, 2014, https://www.eia.gov/.

[2] D. J. Ramberg and J. E. Parsons, "The weak tie between natural gas and oil prices," The Energy Journal, vol. 33, no. 2, 2012.

[3] S. P. A. Brown and M. K. Yücel, "What drives natural gas prices?," The Energy Journal, vol. 29, no. 2, pp. 45-60, 2008.

[4] L. J. Bachmeier and J. M. Griffin, "Testing for market integration crude oil, coal, and natural gas," The Energy Journal, vol. 27, no. 2, pp. 55-71, 2006.

[5] R. F. Engle and C. W. J. Granger, "Co-integration and error correction: representation, estimation, and testing," Econometrica, vol. 55, no. 2, pp. 251-276, 1987.

[6] F. Asche, P. Osmundsen, and M. Sandsmark, "The UK market for natural gas, oil and electricity: are the prices decoupled?," The Energy Journal, vol. 27, no. 2, pp. 27-40, 2006.

[7] P. Hartley, K. B. Medlock, and J. Rosthal, "The relationship between crude oil and natural gas," in Natural Gas in North America: Markets and Security, Energy Forum James A. Baker III, Institute for Public Policy, Rice University, 2007. 
[8] D. J. Ramberg and J. E. Parsons, The Weak Tie between Natural Gas and Oil Prices, CEEPR Working Paper 10-017, Massachusetts Institute of Technology, Cambridge, MA, USA, 2011.

[9] V. Roberta, "Multi-commodity management: the relationship between energy commodities prices," University of St. Gallen, 2011, [M.S. thesis].

[10] J. A. Villar and F. L. Joutz, The Relationship between Crude Oil and Natural Gas Prices, Energy Information Administration, Office of Oil and Gas, 2006.

[11] S. A. Holditch and R. R. Chianelli, "Factors that will influence oil and gas supply and demand in the 21st century," MRS Bulletin, vol. 33, no. 4, pp. 317-323, 2008.

[12] D. J. Ramberg, The relationship between crude oil and natural gas spot prices and its stability over time, [M.S. thesis], Massachusetts Institute of Technology, Cambridge, Massachusetts, 2010.

[13] N. Sebastian and S. Thoenes, What drives natural gas prices? a structural VAR approach, Institute of Energy Economics at the University of Cologne, 2013, EWI Working Paper No. 13/02.

[14] A. Serletis and J. Herbert, "The message in North American energy prices," Energy Economics, vol. 21, no. 5, pp. 471-483, 1999.

[15] A. Serletis and R. Rangel-Ruiz, "Testing for common features in North American energy markets," Energy Economics, vol. 26, no. 3, pp. 401-414, 2004.

[16] O. M. Otunuga, G. S. Ladde, and N. Ladde, "Local lagged adapted generalized method of moments: an innovative estimation and forecasting approach and its applications," Journal Time Series Econometrics, vol. 11, no. 1, 2014.

[17] O. M. Otunuga, G. S. Ladde, and N. G. Ladde, "Local lagged adapted generalized method of moments and applications," Stochastic Analysis and Applications, vol. 35, no. 1, pp. 110$143,2016$.

[18] O. M. Otunuga, "Time varying parameter estimation scheme for a linear stochastic differential equation," International Journal of Statistics and Probability, vol. 6, no. 5, p. 84, 2017.

[19] A. Mummert and O. M. Otunuga, "Parameter identification for a stochastic SEIRS epidemic model: case study influenza," Journal of Mathematical Biology, vol. 79, no. 2, pp. 705-729, 2019.

[20] O. M. Otunuga, Stochastic modeling and analysis of energy commodity spot price processes, University of South Florida, Tampa, FL, USA, 2014, Ph.D. Dissertation.

[21] G. Casella and R. L. Berger, Statistical Inference, Duxbury Advanced Series, Pacific Grove, CA, USA, 2nd edition, 2002.

[22] A. Paothong and G. S. Ladde, "Adaptive expectations and dynamic models for network goods," Economic Analysis and Policy, vol. 43, no. 3, pp. 353-373, 2013.

[23] R. Khasminskii, Stochastic Stability of Differential Equations, Sijthoff \& Noordoff, Rockville, MD, USA, 1980.

[24] G. S. Ladde and V. Lakshmikantham, Random Differential Inequalities, Academic press, New York, NY, USA, 1980.

[25] A. G. Ladde and G. S. Ladde, An Introduction to Differential Equations: Stochastic Modelling Methods and Analysis, vol. 2, World Scientific Publishing Company, Singapore, 2013.

[26] P. E. Kloeden and E. Platen, Numerical Solution of Stochastic Differential Equations, Springer-Verlag, New York, NY, USA, 1992.

[27] G. S. Ladde, V. Lakshmikantham, and A. S. Vatsala, Monotone Iterative Techniques for Nonlinear Differential Equations, Pitman Publishing, Inc., Marshfield, MA, USA, 1985.
[28] D. P. Siu and G. S. Ladde, "Stochastic hybrid system with nonhomogeneous jumps," Nonlinear Analysis: Hybrid Systems, vol. 5, no. 3, pp. 591-602, 2011.

[29] L. Wu, Stochastic Modeling and Statistical Analysis, Dissertation, University of South Florida, 2010.

[30] G. S. Ladde, "Stability and oscillations in single-species processes with past memory," International Journal of Systems Science, vol. 10, no. 6, pp. 621-647, 1979.

[31] R. M. Kulkarni and G. S. Ladde, "Stochastic stability of shortrun market equilibrium: a comment," The Quarterly Journal of Economics, vol. 93, no. 4, pp. 731-735, 1979.

[32] G. E. P. Box, G. M. Jenkins, and G. C. Reinsel, Time Series Analysis Forecasting and Control, Prentice-Hall, Inc., Englewood Cliffs, NJ, USA, 3rd edition, 1994.

[33] T. M. Apostol, Mathematical Analysis, Addison-Wesley Publishing Company, Inc, 1974.

[34] A. G. Ladde and G. S. Ladde, An Introduction to Differential Equations: Deterministic Modelling, Methods and Analysis, vol. 1, World Scientific Publishing Company, Singapore, 2012.

[35] Daily Coal Data Set for the Period 05/04/2009-01/03/2014Arch Coal Incorporationhttp://finance.yahoo.com/q/hp?s=ACI.

[36] Daily Crude Oil West Texas Intermediate (WTI) Spot Price FOB (Dollars per Barrel) Data Set for the Period 05/04/200901/03/2014U. S. Energy Information Administration Website, https://www.eia.gov/.

[37] Daily Henry Hub Natural Gas Data Set (Dollars per Million Btu) for the period 05/04/2009-01/03/2014U. S. Energy Information Administration Websitehttps://www.eia.gov/. 